\title{
THE IMPLICATION OF KV10.1 IN THE REGULATION OF G2/M PROGRESSION
}

\author{
Dissertation for the award of the degree \\ "Doctor rerum naturalium" \\ of the Georg-August-Universität Göttingen
}

within the Molecular Medicine doctoral program of Göttingen Graduate School for Neurosciences, Biophysics, and Molecular Biosciences (GGNB) and Georg-August University School of Science (GAUSS)

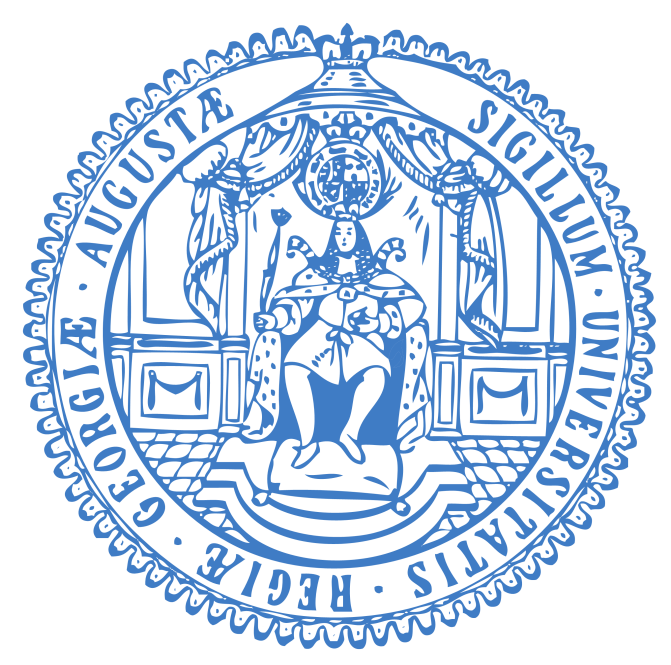

submitted by

Naira Movsisyan

born in Nizhny Novgorod, Russia

from Yerevan, Armenia

Göttingen, 2019 



\section{Members of the Thesis Advisory}

\section{Committee}

\section{Prof Dr Luis A. Pardo (supervisor)}

Max Planck Institute for Experimental Medicine

Oncophysiology Group

Hermann-Rein-Str. 3

37075 Göttingen

Tel.: +49-(0)551-3899643

Fax: +49-(0)551-3899 644

e-mail: pardo@em.mpg.de

\section{Prof Dr Blanche Schwappach $\left(2^{\text {nd }}\right.$ member $)$}

University Medical Centre Göttingen

Department of Molecular Biology

Humboldtallee 23

37073 Göttingen

Germany

Tel.: +49-(0)551-39 5962

Fax: +49-(0)551-39 5960

e-mail: blanche.schwappach@med.uni-goettingen.de

\section{Prof Dr Steven Johnsen ( $3^{\text {rd }}$ member)}

University Medical Centre Göttingen

Clinic for General, Visceral and Paediatric Surgery

UBFT 3-C2-514

Robert-Koch-Straße 40

37075 Göttingen

Tel.: +49 (0)551 39-20830 (Office)

Tel.: +49 (0)551 39-9755 (Assistant: Larissa Geier)

Fax: +49 (0)551 39-12297

e-mail: steven.johnsen@med.uni-goettingen.de 
Members of the Thesis Advisory Committee 


\section{Members of the Examination Board}

\section{Prof Dr Luis A. Pardo (referee)}

Max Planck Institute for Experimental Medicine

Oncophysiology Group

$\underline{\text { Prof Dr Blanche Schwappach (co-referee) }}$

University Medical Centre Göttingen

Department of Molecular Biology

\section{Prof Dr Steven Johnsen}

University Medical Centre Göttingen

Clinic for General, Visceral and Paediatric Surgery

Other members of the examination board:

\section{Prof Dr Dieter Kube}

University Medical Centre Göttingen

Department of Haematology and Oncology

\section{$\underline{\text { Prof Dr Manuela Schmidt }}$}

Max Planck Institute for Experimental Medicine

Somatosensory Signaling Group

\section{Dr Dieter Klopfenstein}

Third Institute of Physics

Department of Biophysics

\section{Alexis Faesen, PhD}

Max Planck Institute of Biophysical Chemistry, Göttingen

Biochemistry of Signal Dynamics Group 
Members of the Examination BoARd 


\section{AfFidAVIT}

I hereby assure that I have composed the present doctoral thesis entitled

"The Implication of Kv10.1 in the Regulation of G2/M Progression"

independently and I have used no other appliances than indicated. Parts being gathered from other works according to wording or meaning I have stated in every single case by the declaration of the source.

I hereby state furthermore, that I have produced my work according to the principles of good scientific practise in compliance with the valid "Richtlinien der Georg-August-Universität Göttingen zur Sicherung guter wissenschaftlicher Praxis".

$31^{\text {st }}$, March 2019

Naira Movsisyan 
AfFidAVIT 
To my family and my beloved niece and nephew 


\section{Acknowledgements}

I would like to thank all the people who have helped and supported me throughout this exciting journey be it related to the work described in my thesis or, in general.

First and foremost, I would like to express my sincere gratitude to my supervisor Prof Dr Luis Pardo for giving me the opportunity to continue this fascinating project which I started and absolutely loved during my Master studies. I can call myself "lucky" in terms of having him as my supervisor and mentor. He gave me all the freedom I needed and flourished the creativity. Yet his door was always open for discussions and any support sought throughout three and a half years of my doctoral studies,

I would like to thank both my thesis committee members Prof Dr Blanche Schwappach and Prof Dr Steven Johnsen for the critical discussions, insightful comments and suggestions, which helped me to keep the project on a track and not to jump onto something like "this sounds interesting, maybe I should check it?".

My sincere gratitude to Prof Dr Walter Stühmer for all his help and encouragement, and all the interesting stories and facts he told us over coffee and get-together. Many thanks to Dr Diana Urrego for her guidance and the discussions in the first year of the project; Dr Jorge Fernández-Trillo for lending me the super-comfy air mattress for the overnight synchronisation experiments. My deepest gratitude to Dr Farrah Zahed for being a true friend over these years and, of course, all her help. Special thanks to Dr Ileana Hernández-Reséndiz for stimulating discussions and for all the support needed in the lab and outside. Many thanks to Ute Rust for all the administrative help. My sincere and deepest gratitude to Jan Seidel for helping me with calcium imaging data analysis, in particular, writing the $\mathrm{R}$ script, compiling my doctoral thesis and all the support and encouragement throughout this time. I would like to thank all the former and current members of Oncophysiology group, Reham Abdelaziz, Araceli Sánchez, Kerstin Dümke, Franziska Hartung, Victor Diaz, and the department of "Molecular biology of neuronal signals". Without their precious support and knowledge, it would not be possible to conduct this research. 
Many thanks to all my friends from "Molecular Medicine" programme and Armenian community, especially Nelly, who always welcomed all of us and made feel like being back in Armenia. I am grateful to Dr Sumon Sur for all his help and support over these years.

Finally, I would like to express my deepest gratitude to my family who I know are always behind my back and no matter what, I will always have that safe harbour. They taught me to never panic, never give up and believe that everything is doable. I would have not been writing this now without their unconditional love and care.

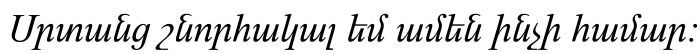




\section{Table of Contents}

Members of the Thesis Advisory Committee i

Members of the Examination Board iii

$\begin{array}{lll}\text { Affidavit } & \text { v }\end{array}$

Acknowledgements $\quad$ ix

Table of Contents $\quad$ xi

List of Figures $\quad$ XV

List of Tables $\quad$ xvii

Abbreviations $\quad$ xix

$\begin{array}{ll}\text { Abstract } & \text { xxv }\end{array}$

1 Introduction $\quad 1$

1.1 Structure and gating mechanism of Kv10.1 . . . . . . . . . . . . 1

1.2 Role and regulation of Kv10.1 in non-cancer and cancer cells . . . . . 5

1.2.1 Non-cancer cells . . . . . . . . . . . . . . . . . . 5

1.2 .2 Cancer cells . . . . . . . . . . . . . . . . . 10

1.3 Cell cycle: stages and checkpoints . . . . . . . . . . . . . 17

1.3.1 Overview of the cell cycle . . . . . . . . . . . . . . 17

1.3.2 Regulation of the G1/S transition: pRb-E2F axis . . . . . . . 19

1.3.3 The DNA damage-induced checkpoint . . . . . . . . . . 20

1.3.4 The mitotic spindle assembly checkpoint . . . . . . . . . . 25

1.4 Microtubules ........................ 28

1.4.1 The dynamic instability of microtubules . . . . . . . . . . 28

1.4.2 Microtubules in the presence of calcium . . . . . . . . . . . . 31

The Aim of the Work 35 
TABle of Contents

2 Materials \& Methods $\quad 37$

2.1 Cell culture . . . . . . . . . . . . . . . . . . 37

2.1 .1 Cell lines. . . . . . . . . . . . . . . . . 37

2.1.2 Passaging of cells . . . . . . . . . . . . . . 37

2.1.3 Cell count ....................... 37

2.1.4 Plasmid DNA purification . . . . . . . . . . . . 38

2.1.5 siRNA and pDNA transfection . . . . . . . . . . . . 39

2.1.6 Cell cycle synchronisation . . . . . . . . . . . . . 42

2.2 Biochemistry . . . . . . . . . . . . . . . 46

2.2.1 Protein extraction . . . . . . . . . . . . . 46

2.2.2 Protein concentration determination . . . . . . . . . . 46

2.2 .3 SDS-PAGE . . . . . . . . . . . . . . 47

2.2 .4 Immunoblotting . . . . . . . . . . . . . . . . . . . 47

2.2.5 Immunoprecipitation . . . . . . . . . . . . . . . . 49

2.2.6 Densitometry . . . . . . . . . . . . . . . . 50

2.3 Single-cell gel electrophoresis . . . . . . . . . . . . . . . . . . . 51

2.4 Immunofluorescence staining . . . . . . . . . . . . . . . 55

2.4.1 Quantification of asymmetric mitotic spindles, defective metaphases and lagging chromosomes . . . . . . . . . . . 57

2.4.2 Determination of mitotic spindle angle . . . . . . . . . . 57

2.5 Determination of PLK1 enzymatic activity . . . . . . . . . . 58

2.6 Proximity ligation assay . . . . . . . . . . . . . . . . 60

2.7 Live cell imaging . . . . . . . . . . . . . . . . . . . 62

2.7.1 Assessment of microtubule dynamics . . . . . . . . . . . . 62

2.7.2 Intracellular calcium imaging . . . . . . . . . . . . . 69

2.8 Statistical analysis . . . . . . . . . . . . . . . . 73

$\begin{array}{lll}3 & \text { Results } & 75\end{array}$

3.1 Kv10.1 and DNA damage-induced checkpoint . . . . . . . . . . . 76

3.1.1 Downregulation of Kv10.1 partially activates DNA damageinduced checkpoint . . . . . . . . . . . 76

3.1.2 The activation of DDR in Kv10.1-deficient cells does not obey actual DNA damage . . . . . . . . . . . . . . 77 
3.2 Kv10.1 and Spindle Assembly Checkpoint . . . . . . . . . . . . . 79

3.2.1 SAC is activated in Kv10.1-deficient HeLa cells . . . . . . . . 79

3.2.2 Activation of SAC in Kv10.1-deficient cells prevents the formation of lagging chromosomes during anaphase onset . . . . 81

3.2.3 Kv10.1 knockdown results in higher PLK1 activity . . . . . . 83

3.2.4 Altered mitotic spindle angle is observed upon loss of Kv10.1 . 85

3.3 Kv10.1 and microtubule dynamics . . . . . . . . . . . . . . 86

3.3.1 Downregulation of Kv10.1 increases the occurrence of asymmetric monopolar mitotic spindles in HeLa cells . . . . . . . . 86

3.3.2 The absence of Kv10.1 results in an increased MT plus-end growth ....................... 87

3.3.3 Overexpression of Kv10.1 has opposite effects to Kv10.1 knockdown on MT dynamics in HeLa cells . . . . . . . . . . . . . . 89

3.4 Kv10.1 and ORAI1 functional interaction . . . . . . . . . . . . . . 90

3.4.1 Kv10.1 and ORAI1 are in close proximity to each other . . . . 90

3.4.2 Silencing of Kv10.1 decreases the intracellular $\left[\mathrm{Ca}^{2+}\right]$ in hTERT RPE1 cells . . . . . . . . . . . . . . . . 91

3.4.3 Loss of Kv10.1 reduces the frequency of cytosolic calcium oscillations . . . . . . . . . . . . . . . . . 93

3.5 Importance of conductance of Kv10.1 and ORAI1 in the regulation of MT dynamics . . . . . . . . . . . . . . . . . . . 95

3.5.1 Astemizole-mediated blockade of Kv10.1 conductance reduces MT dynamics in HeLa cells . . . . . . . . . . . . . . 95

3.5.2 Monoclonal antibody-mediated blockade of Kv10.1 conductance decreases MT dynamics in HeLa cells . . . . . . . . . 97

3.5.3 Inhibition of ORAI1 conductance mimics the effects of inhibition of Kv10.1 current on MT dynamics . . . . . . . . . . 98

4 Discussion

4.1 Kv10.1 knockdown results in the partial activation of DNA damageinduced checkpoint in the absence of DNA damage . . . . . . . . . . 101

4.2 Kv10.1 downregulation enhances the SAC response and prevents the formation of lagging chromosomes . . . . . . . . . . . . . . 103 
TABle of Contents

4.3 Kv10.1-deficient cells display tilted mitotic spindle orientation . . . . 106

4.4 Microtubule dynamics increase when Kv10.1 is downregulated . . . . 107

4.5 Kv10.1 and ORAI1 team up: downregulation of Kv10.1 reduces the cytosolic calcium concentration and oscillations . . . . . . . . 108

4.6 Inhibition of the conductance of either Kv10.1 or ORAI1 reduces MT

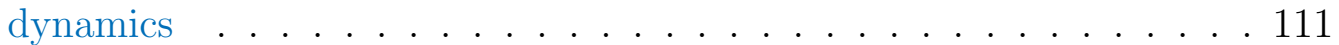

5 Conclusion \& Perspectives

References

115

Appendix

169

R code for calcium data processing . . . . . . . . . . . . . . 171

MATLAB code for calcium oscillations analysis . . . . . . . . . . 173 


\section{List of Figures}

Figure $1.1 \ldots \ldots \ldots \ldots \ldots$. . . . . . . . . . . . . . . . 2

Figure $1.2 \ldots \ldots \ldots \ldots \ldots$

Figure $1.3 \ldots \ldots \ldots \ldots \ldots \ldots$

Figure $1.4 \ldots \ldots \ldots \ldots \ldots \ldots$

Figure $1.5 \ldots \ldots \ldots \ldots \ldots \ldots$

Figure $1.6 \ldots \ldots \ldots \ldots \ldots \ldots$

Figure $1.7 \ldots \ldots \ldots \ldots \ldots \ldots$

Figure $1.8 \ldots \ldots \ldots \ldots \ldots \ldots \ldots$

Figure $1.9 \ldots \ldots \ldots \ldots$

Figure $1.10 \ldots \ldots \ldots \ldots$

Figure $1.11 \ldots \ldots \ldots \ldots \ldots \ldots \ldots$

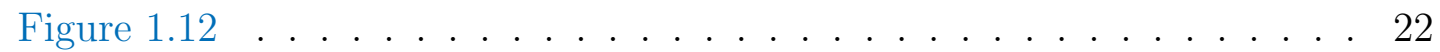

Figure $1.13 \ldots \ldots \ldots \ldots \ldots \ldots \ldots$

Figure $1.14 \ldots \ldots \ldots \ldots \ldots \ldots$

Figure $1.15 \ldots \ldots \ldots \ldots \ldots \ldots$

Figure $1.16 \ldots \ldots \ldots \ldots \ldots$

Figure $2.1 \ldots \ldots \ldots \ldots \ldots \ldots$

Figure $2.2 \ldots \ldots \ldots \ldots \ldots$

Figure $2.3 \ldots \ldots \ldots \ldots \ldots$

Figure $2.4 \ldots \ldots \ldots \ldots \ldots \ldots \ldots$

Figure $2.5 \ldots \ldots \ldots \ldots \ldots \ldots$

Figure $2.6 \ldots \ldots \ldots \ldots$

Figure $2.7 \ldots \ldots \ldots \ldots \ldots$

Figure $2.8 \ldots \ldots \ldots \ldots \ldots \ldots \ldots$

Figure $2.9 \ldots \ldots \ldots \ldots \ldots \ldots$

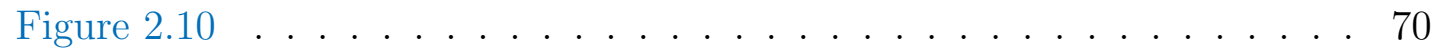

Figure $2.11 \ldots \ldots \ldots \ldots \ldots$. . . . . . . . . . . . . . . . . . . . . . . . . 
List of Figures

Figure $3.1 \ldots \ldots \ldots \ldots \ldots$. . . . . . . . . . . . . . . . . . . . . . . . .

Figure $3.2 \ldots \ldots \ldots \ldots \ldots$

Figure $3.3 \ldots \ldots \ldots \ldots$. . . . . . . . . . . . . . . . . 80

Figure $3.4 \ldots \ldots \ldots \ldots$. . . . . . . . . . . . . . . . . . 82

Figure $3.5 \ldots \ldots \ldots \ldots \ldots$. . . . . . . . . . . . . . . . 84

Figure $3.6 \ldots \ldots \ldots \ldots$

Figure $3.7 \ldots \ldots \ldots \ldots \ldots$. . . . . . . . . . . . . . . . . . . . . . .

Figure $3.8 \ldots \ldots \ldots \ldots \ldots \ldots$

Figure $3.9 \ldots \ldots \ldots$

Figure $3.10 \ldots \ldots \ldots \ldots$. . . . . . . . . . . . . . . . . . . 91

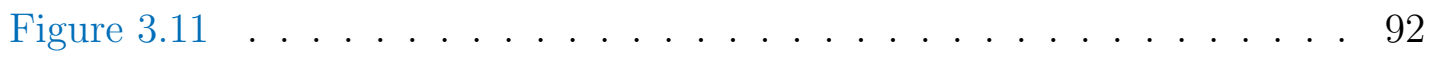

Figure $3.12 \ldots \ldots \ldots \ldots$

Figure $3.13 \ldots \ldots \ldots \ldots \ldots \ldots$

Figure $3.14 \ldots \ldots \ldots \ldots$. . . . . . . . . . . . . . . . . . 96

Figure $3.15 \ldots \ldots \ldots \ldots$

Figure $3.16 \ldots \ldots \ldots$. . . . . . . . . . . . . . . . . . . . . . 99 


\section{List of Tables}

Table $1.1 \ldots \ldots \ldots . \ldots . \ldots \ldots$

Table $2.1 \ldots \ldots \ldots \ldots$

Table $2.2 \ldots \ldots \ldots \ldots \ldots \ldots \ldots$

Table $2.3 \ldots \ldots \ldots \ldots \ldots \ldots$

Table $2.4 \ldots \ldots \ldots \ldots$

Table $2.5 \ldots \ldots \ldots \ldots \ldots \ldots \ldots$ 
List of TABLES 


\section{Abbreviations}

ANOVA analysis of variance

AP action potential

APC/C anaphase-promoting complex/cyclosome

ATM ataxia telangiectasia mutated kinase

ATR ATM and Rad3 related kinase

AVD apoptotic volume decrease

BAX Bcl2-associated X protein

Best1 bestrophin 1

Bgap backward gap

Bora aurora borealis

BRCA1 breast cancer 1, early onset

BSA bovine serum albumin

BUB3 budding uninhibited by benzimidazoles 3 homolog

BUBR1 BUB1-related protein 1

$\mathrm{Ca}^{2+} \quad$ calcium ions

$\left[\mathrm{Ca}^{2+}\right] \quad$ calcium ion concentration

CaM calmodulin

CaMBD calmodulin-binding domain

CaMKII calcium/CaM-dependent protein kinase II

$\mathrm{CaN} \quad$ calcineurin

CDC cell division cycle

CDK cyclin-dependent kinase

CENP centromere protein

CHK checkpoint kinase

CHO Chinese hamster ovary

ch-TOG colonic and hepatic tumour overexpressed gene protein

CKI cyclin-dependent kinase inhibitors

CNBHD cyclic nucleotide-binding homology domain

CPC chromosomal passenger complex 


$\begin{array}{ll}\text { Cryo-EM } & \text { cryo-electron microscopy } \\ \text { CTCF } & \text { corrected total cell fluorescence } \\ \text { CTTN } & \text { cortactin } \\ \text { ddH }_{2} \mathrm{O} & \text { deionised distilled water } \\ \text { DDR } & \text { DNA damage response } \\ \text { DME } & \text { dimethylenastron }\end{array}$

DMEM/F-12 Dulbecco's Modified Eagle Medium: Nutrient Mixture F-12

DoG Difference of Gaussians

DPBS Dulbecco's Phosphate Buffered Saline

DSB double-strand break

EAG Ether-à-go-go

EB3 end-binding protein 3

ECM extracellular matrix

EGFR epidermal growth factor receptor

EGTA ethylene glycol-bis(beta-aminoethyl)-N,N,N',N'-tetraacetic acid

EMT epithelial-to-mesenchymal transition

FAK focal adhesion kinase

FCS fetal calf serum

Fgap forward gap

FWHM full width at half maximum

G gap

GUI graphical user interface

h human

HDAC histone deacetylase

HEF1 enhancer of filamentation 1

hERG1 human EAG related gene 1

HIF1 $\alpha$ hypoxia-inducible factor $1 \alpha$

HPV human papillomavirus

HRP horseradish peroxidase

INCENP inner centromere protein

$\mathbf{K}^{+} \quad$ potassium ions

KCNH1 potassium voltage-gated channel subfamily $\mathrm{H}$ member 1 gene 
KD knockdown

KIF kinesin family member

KO knockout

Kv channels voltage-gated potassium channels

LB Luria Broth

LMP low melting point

M mitotic

MAD2L1 mitotic arrest deficient 2 like 1

MAP microtubule-associated protein

MAPK mitogen-activated protein kinase

MCAK mitotic centromere-associated kinesin

MCC mitotic checkpoint complex

MDC1 mediator of DNA damage checkpoint protein 1

MDM2 mouse double minute 2 homolog

MPF mitosis-promoting factor

MPS1 monopolar spindle 1

MT microtubule

MTOC MT-organising centre

N.A. numerical aperture

NC nitrocellulose

NEB nuclear envelope breakdown

NEDD9 neural precursor cell expressed, developmentally downregulated 9

NES nuclear export sequence

NLS nuclear localisation sequence

NMP normal melting point

ORAI1 calcium release-activated calcium channel protein 1

OTM olive tail moment

p- phosphorylated

PAS Per-Arnt-Sim

PCM pericentriolar matrix

PD pore domain

pfa paraformaldehyde 
PIP2 phosphatidylinositol 4,5-bisphosphate

PLA proximity ligation assay

PLK1 Polo-like kinase 1

P-loop pore loop

pRb retinoblastoma protein

pVHL von Hippel-Lindau protein

RAB Ras-related in brain

RCA rolling circle amplification

RNR1 ribonucleotide reductase 1

ROIs regions of interest

RPMI Roswell Park Memorial Institute

RT room temperature

SAC spindle assembly checkpoint

Scr $\quad$ scrambled

SD standard deviation

SDS-PAGE sodium dodecyl sulphate polyacrylamide gel electrophoresis

SEM standard error of the mean

siRNA small interfering RNA

SSB single-strand break

SV40 simian virus 40

TCC tetramerising coiled coil

TIFF tag image format file

+TIPs plus-end-tracking proteins

-TIPs minus-end-tracking proteins

TM transmembrane

TMo tail moment

TRAIL TNF-related apoptosis inducing ligand

TRT telomerase reverse transcriptase

VEGF vascular endothelial growth factor

vs versus

VSD voltage-sensing domain

WB western blot 
AbBreviations

WT

wild type

XMAP

Xenopus microtubule-associated protein 
AbBreviations 


\section{Abstract}

Kv10.1, also termed EAG1 (Ether-à-go-go1), is a voltage gated potassium channel, initially thought to be expressed in the central nervous system. Prolonged or increased expression of Kv10.1 has been found in over $70 \%$ of all human tumour tissues, where its presence correlates with poorer prognosis. In peripheral tissues, Kv10.1 is expressed specifically during the G2/M phase of the cell cycle, and downregulation of the channel extends the G2/M phase duration both in cancer and normal cells. In this project, we elucidate the mechanisms of Kv10.1-mediated regulation at the G2/M phase. We show that Kv10.1 has a dual effect on mitotic microtubule dynamics. It interacts functionally with ORAI1 and through modulation of the cytosolic calcium oscillations changes the microtubule behaviour. Inhibition of either Kv10.1 or ORAI1 conductance stabilises the microtubules. In contrast, siRNAmediated downregulation of Kv10.1 increases the dynamicity of mitotic microtubules, resulting in a stronger spindle assembly checkpoint, greater mitotic spindle angle, and a decrease in the occurrence of lagging chromosomes.

In general, understanding of Kv10.1 modulation of the microtubule architecture will help to find out how exactly a cancer tissue benefits from the presence of Kv10.1.

Keywords: Kv10.1, cell cycle, G2/M phase, DNA damage activated-checkpoint, spindle assembly checkpoint, microtubule dynamics, ORAI1, calcium. 
Abstract 


\section{Chapter 1}

\section{Introduction}

\subsection{Structure and gating mechanism of Kv10.1}

Ion channels are transmembrane proteins, which passively transport specific inorganic ions between cell membranes down their electrochemical gradient. An electrochemical gradient is a result of the asymmetrical distribution of ions across cell membranes, and it is maintained by ATP-pumps (Subramanyam \& Colecraft, 2015).

Ion channels are classified depending on the nature of gating, the selectivity of ion permeation, the number of gates (pores) and localisation of proteins (Hille, 2001). Among them are potassium channels - transmembrane (TM) proteins defined by a high affinity for $\mathrm{K}^{+}$transport and present virtually in all cells.

Potassium channels are formed by principal subunits ( $\alpha$-subunits), which determine channel structure, and auxiliary subunits ( $\beta$-subunits), which modify channel properties (Rettig et al., 1994). More than 90 genes in human genome encode $\alpha$-subunits of potassium channels, which depending on the structure and function are grouped into eight families (Wei, Jegla, \& Salkoff, 1996). These families are further subdivided into the following groups: i) calcium-activated six/seven TM, ii) voltage-gated six TM and iii) two TM potassium channels (Tian et al., 2014).

Voltage-gated potassium channels (Kv channels) make up the considerable portion of the ion channels and are encoded by 40 genes. One of such channels is Kv10.1 or often termed EAG1 (Ether-à-go-go 1). It is a member of the EAG family and is encoded by the KCNH1 gene (potassium voltage-gated channel subfamily H member 1 gene), located on chromosome 1q32.1-32.3 (Gutman et al., 2005; Occhiodoro et al., 1998). Kv10.1 shares about $70 \%$ identity in amino acid sequence with another member of the EAG family, Kv10.2 (EAG2, KCNH5; Ju \& Wray, 2002).

Structurally, the EAG family is similar to other Kv channels. The functionally active channel is a tetramer made of four $\alpha$-subunits arranged circumferentially and 
forming the central conduction pore. Each $\alpha$-subunit comprises of six, S1-S6 TM segments (Figure 1.1). The S5 and S6 segments form the ion-permeation pore (pore

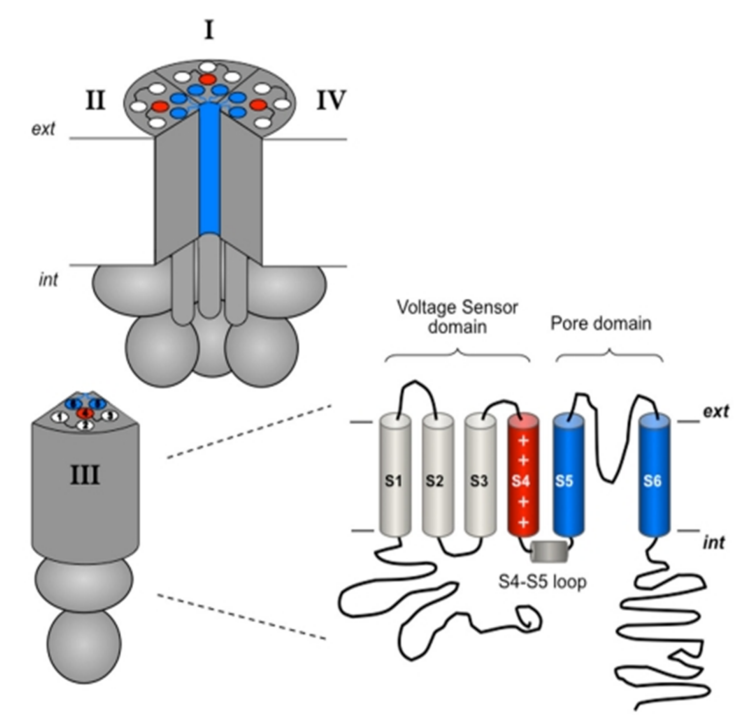

Figure 1.1: Schematic representation of the tetrameric organisation of a $K v$ channel. All four $\alpha$-subunits are arranged circumferentially with their S5 and S6 segments constituting the permeation pore (top). A structural folding model and a simplified view of one of the four $\alpha$-subunits are shown (bottom). Adapted from (Barros, Domínguez, \& de la Peña, 2012). domain, PD) with the activation gate localised at the cytoplasmic end of the S6 segment. The S5 and S6 segments are linked by an S5-S6 pore loop (P-loop). The second gate of the channel, ion selectivity filter gate is localised in the $\mathrm{P}$ loop. The S1-S4 segments constitute the voltage-sensing domain (VSD), where S4 is the voltage-sensing component and is enriched with positively charged residues, such as arginine and lysine (Barros et al., 2012; Bezanilla, 2008; Stühmer et al., 1989; Swartz, 2004; Yellen, 2002). The PD and VSD are connected by the S4-S5 linker. It was proposed that changes in membrane potential are sensed by the S4 segment resulting in the movement of the VSD coupled to the S4-S5 linker; the latter acts as a lever on the S6 segment and, thus, mechanically closes the pore (Long, Campbell, \& Mackinnon, 2005a, 2005b; Long, Tao, Campbell, \& MacKinnon, 2007).

However, experiments utilising "split" channels constituting of separate fragments of VSD and PD show that the covalent bond with the S4 segment affects the closing properties of the channel at negative potentials, whereas disconnecting from S5 segment attenuates the activation and deactivation kinetics. Overall, we suggest that an inward movement of the S4 helix, but not the S4-S5 linker, closes the channel, while also proposing that activation of the channel by the voltage sensor involves more than one mechanism (Tomczak et al., 2017).

Moreover, our suggested model is in agreement with the recently published singleparticle cryo-EM (cryo-electron microscopy) structure of mammalian Kv10.1 in its inactive, bound to CaM (calmodulin) form (Figure 1.2; Whicher \& MacKinnon, 


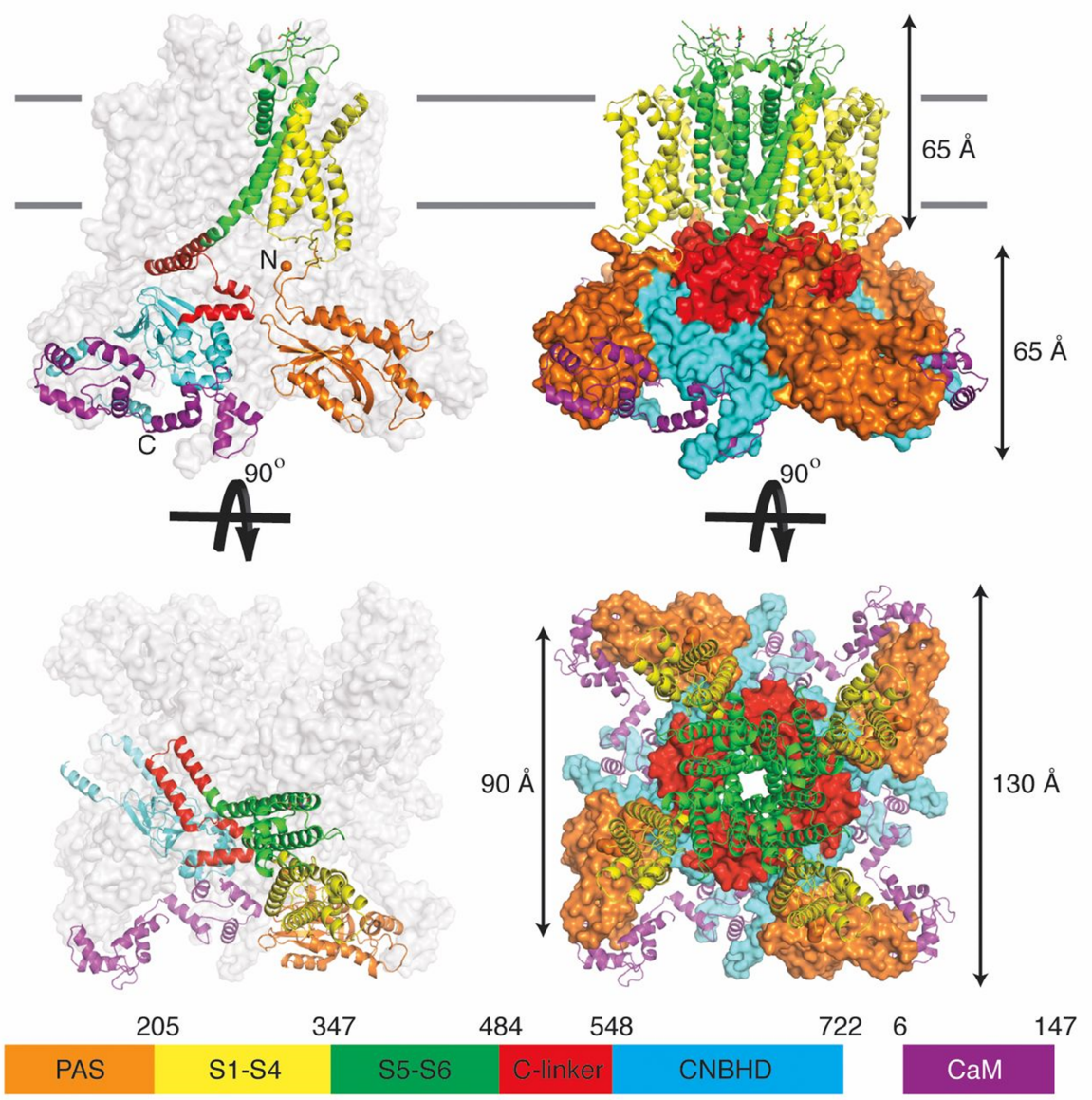

Figure 1.2: Model of rat Kv10.1 structure bound to CaM based on single-particle cryo-EM. The domains of the channel are colour-coded as indicated in the panel given at the bottom. The positioning of only one subunit within the functional channel is shown in colour on the left as cartoons. On the right the transmembrane (shown as a cartoon) and the cytoplasmic domains (shown as a surface) are given with indicated length and width. The $N$ - and $C$ - termini are labelled in orange and cyan, respectively. The grey lines indicate the cytoplasmic membrane. Adapted from (Whicher 83 MacKinnon, 2016).

2016). Unlike other Kv channels, the S4-S5 linker of Kv10.1 contains only five amino acids, thus affecting its structure and positioning. The latter facilitates CaM binding and channel pore closing independently of the VSD (Whicher \& MacKinnon, 2016).

The EAG family is also characterised by long $\mathrm{N}$ - and C-terminal cytoplasmic domains (Wray, 2004) as illustrated in Figure 1.3. The C-terminal tail is located directly beneath the TM domain (Barros et al., 2012). It consists of a CNBHD (cyclic nucleotide-binding homology domain), which, however, has no affinity for cyclic nucleotide binding with only exception of the Drosophila variant (Brüggemann, Pardo, Stühmer, \& Pongs, 1993; Marques-Carvalho et al., 2012). The C-terminus also has TCC (tetramerising coiled coil) domain. The TCC is involved not only in the correct tetramerisation but also in the stability and selectivity of the multimerisation (Jenke 


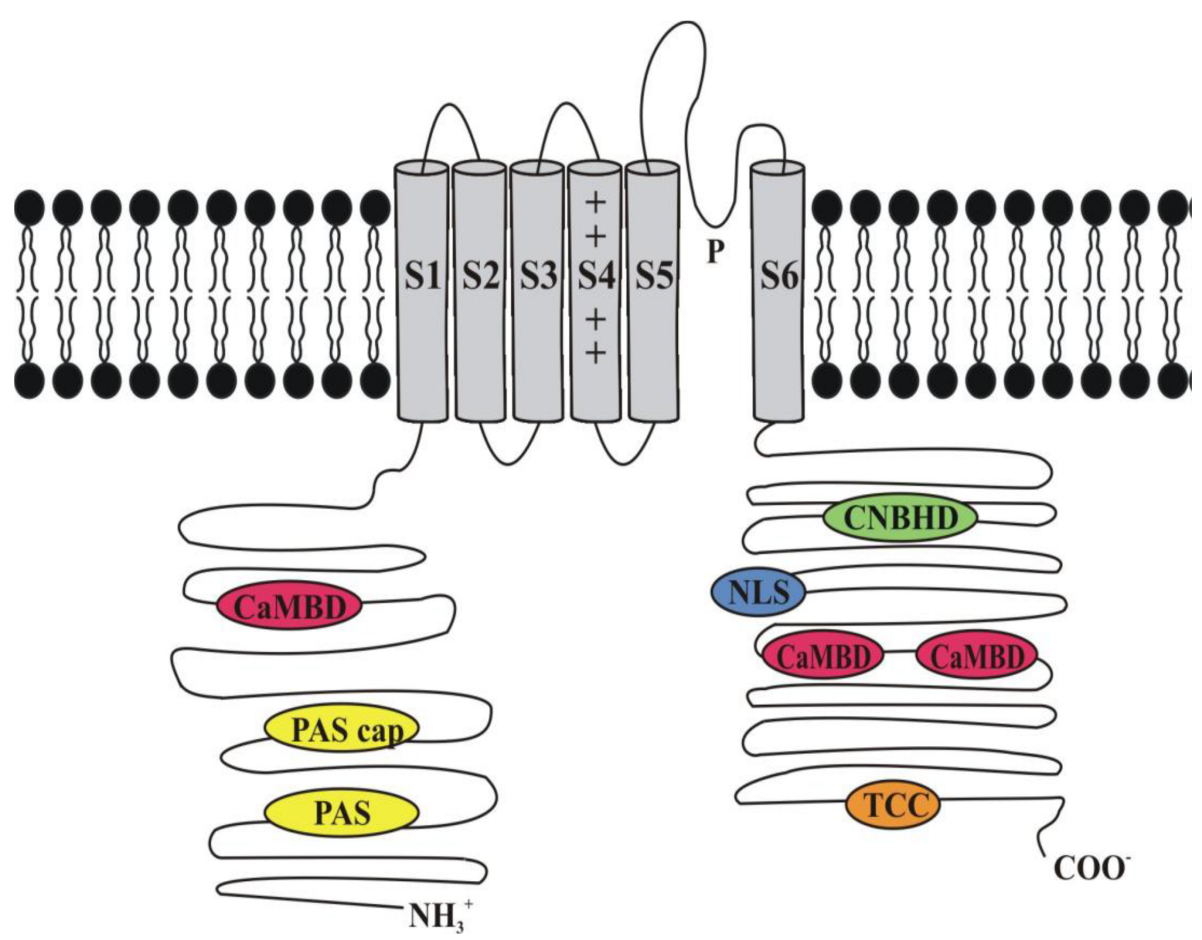

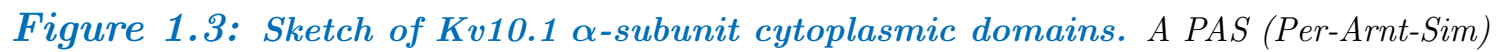
domain and its preceding PAS-cap, and a CaMD (calmodulin-binding domain) are localised at the $N$-terminus. At the C-terminus, a CNBHD (cyclic nucleotide-binding homology domain), a NLS (nuclear localisation signal), CaMBD, and a TCC (tetramerising coiled coil) domain are localised. Adapted from (Urrego-blanco, 2014).

et al., 2003). A binding site for CaMKII (calcium/CaM-dependent protein kinase II) has been identified at the C-terminus of the Drosophila Eag. This interaction results in a stable complex between CaMKII and Eag and activation of CaMKII independently of CaM and autophosphorylation (Sun, Hodge, Zhou, Nguyen, \& Griffith, 2004; Z. Wang, Wilson, \& Griffith, 2002). Also, multiple signalling modules, such as NES (nuclear export sequence) and NLS (nuclear localisation sequence) are present on the C-terminus of Drosophila Eag. It has been suggested that the NES and NLS may play an essential role in perinuclear localisation of these channels (Chen et al., 2011).

The N-terminal tail of Kv10.1 contains a PAS (Per-Arnt-Sim) domain (Adaixo, Harley, Castro-Rodrigues, \& Morais-Cabral, 2013; Haitin, Carlson, \& Zagotta, 2013). PAS domains are suggested to function as sensors during some cell signalling events, for instance, hypoxia (McIntosh, Hogenesch, \& Bradfield, 2010). Several studies have demonstrated that the utmost region of the C-terminus and the PAS domain are involved in controlling the gating kinetics of the EAG channels (Stevens, Ju, \& Wray, 2009; Terlau, Heinemann, Stühmer, Pongs, \& Ludwig, 1997; Wray, 2009). Thus, the 
control of EAG channel gating also involves cytoplasmic domains of the channel. Summarising, these intracellular domains are indispensable for the functioning of the channel. Moreover exposure of these domains to different cytoplasmic cues can alter channel behaviour.

\subsection{Role and regulation of Kv10.1 in non-cancer and cancer cells}

\subsubsection{Non-cancer cells}

\section{Neurones}

The discovery of the EAG family was first associated with the observation of trembling appendages in etherised D. funebris (Luers, 1936) and D. melanogaster males (Catsch, 1948). Catsch named the gene locus responsible for the mutation Shaker. Later, it was shown that this locus comprises a gene complex: three different gene loci located on the X chromosome in Drosophila, including Ether-à-go-go (Kaplan \& Trout, 1969). Further, through electrophysiological studies it was demonstrated that the Shaker genes encode potassium channels with rapidly inactivating voltage sensitive currents (de la Pompa, 1994; Kamb, Tseng-Crank, \& Tanouye, 1988; Papazian, Schwarz, Tempel, Jan, \& Jan, 1987; Tempel, Papazian, Schwarz, Jan, \& Jan, 1987; Warmke, Drysdale, \& Ganetzky, 1991). Subsequently, the excitability of neurones carrying different mutations of Eag1 channel were intensively studied. It was shown that the motor neurones bearing mutations in Eag1 gene spontaneously fired repetitive action potentials and were described with increased transmitter release (Han, Tokay, Zhang, Sun, \& Hou, 2017).

Normally, in mammals, Kv10.1 is detected only in the central nervous system (Occhiodoro et al., 1998). For instance, in rats, the olfactory bulb, cerebral cortex, hippocampus, hypothalamus, and cerebellum are positive for Kv10.1, which substantially overlaps with the regions characterised for humans (S. Martin et al., 2008). Initially, post-synaptic localisation of Kv10.1 was suggested (Jeng, Chang, \& Tang, 2005; Jow \& Jeng, 2008). However, Gómez-Varela et al. proposed the possible pre-synaptic localisation of the channel; using single-particle-tracking techniques 
with quantum dots, they demonstrated that the endogenous Kv10.1 laterally diffuses within the pre-synaptic membrane of neurones isolated from hippocampus of E18 rats (Gómez-Varela et al., 2010). Later, this pre-synaptic localisation of Kv10.1 was also confirmed by Mortensen et. al. The authors showed that Kv10.1 is specifically expressed in the pre-synaptic terminals of the parallel-fibre Purkinje cell synapse, and it does not contribute to the somatic AP (action potentials) in the cerebellum. However, when applying repetitive stimulations the $\mathrm{Ca}^{2+}$ influx into axonal boutons was increased in Kv10.1 KO (knockout), whereas a single stimulation did not have any effect. The $\mathrm{Ca}^{2+}$ release dependency on the frequency of stimuli indicates that the Kv10.1 acts as a modulator of local AP specifically during high-frequency burst firing when other potassium channels are inactive. Thereby, Kv10.1 may shape the excitability and firing dynamics of neurones, such as of Purkinje cells (Mortensen et al., 2015).

Kv10.1 may also be involved in the action of antidepressants (Dreixler et al., 2000; García-Ferreiro et al., 2004b; Mathie, Wooltorton, \& Watkins, 1998). Imipramine, an important antidepressant agent, modulates the aminergic transmission in the central nervous system (Deng, Luo, Vorperian, Petzold, \& Nelson, 2014; Knapp, Breese, Mueller, \& Breese, 2001). In sympathetic neurones, imipramine preferentially blocks delayed-rectifier $\mathrm{K}^{+}$currents, including Kv10.1 (Wooltorton \& Mathie, 1993). This blockade is voltage-dependent and counteracted by intracellular tetraethylammonium, indicating that the imipramine binding site is localised at the inner end of the selectivity filter (García-Ferreiro et al., 2004b).

Genome-wide genetic linkage analysis identified a susceptibility locus for schizophrenia on chromosome 1q32 region (Gurling et al., 2001), similar to the KCNH1 gene (Occhiodoro et al., 1998). Interestingly, histological and electrophysiological examination of the cerebellum of $K C N H 1 \mathrm{KO}$ mice revealed clear differences compared to the WT mice (Ufartes et al., 2013). However, the KCNH1 KO mice showed only mildly hyperactive, but otherwise normal behaviour and no sign of schizophrenic-like aberrations (Ufartes et al., 2013). 


\section{Myoblasts}

Myogenesis is a strictly regulated process of differentiation of fibroblasts into highly ordered multinucleated muscle. The first step of myogenesis is the commitment to a myoblast phenotype, followed by formation of multinucleated myotubes through myoblast fusion, and maturation of myotubes into muscles (E. Cooper, 2001). Myoblast fusion is accompanied with irreversible cell cycle arrest, which is tightly controlled by environmental cues (Chal \& Pourquié, 2017; Hindi, Tajrishi, \& Kumar, 2013). Interestingly, changes in membrabe potential, such as hyperpolarisation, can precede or accompany myoblast fusion. The hyperpolarisation of the membrane is attributed to two types of $\mathrm{K}^{+}$currents - a non-inactivating delayed rectifier and an inward rectifier (J. H. Liu et al., 1998). The non-inactivating delayed rectifier $\mathrm{K}^{+}$ current is akin to the current of rat Eag channels (Bijlenga et al., 1998; Occhiodoro et al., 1998). Moreover, ectopic expression of human KCNH1 in undifferentiated myoblasts generates a current greatly mimicking the one observed during myoblast fusion (Bijlenga et al., 1998; Occhiodoro et al., 1998). These findings indicate implication of Kv10.1 in muscle fibre development.

\section{Disassembly of the primary cilium}

Virtually all quiescent cells have a primary cilium, an antenna-like solitary structure protruding into the extracellular matrix. The primary cilium, initially thought to be a rudimental structure, is a conserved MT (microtubule)-based organelle critical for sensing and reacting to the changes in chemical and mechanical properties of the extracellular environment. Depending on the cues, the cell will change its behaviour, for instance, cell polarity, proliferation or differentiation. The protruding part of the cilium, termed the axoneme, is formed by MTs. The length of the axoneme is regulated by modulating the MT polymerisation and depolymerisation (Mirvis, Stearns, \& James Nelson, 2018).

The primary cilium is assembled on the mother centriole (Nam, Naylor, \& van Deursen, 2015). On the other hand, in dividing cells, centrioles serve as an MTOC (MT-organising centre), which requires detachment of the mother centriole from the plasma membrane. Therefore, the primary cilium and the mitotic spindle are mutually exclusive (I. Sánchez \& Dynlacht, 2016). Thus, during cell division the cilium 
undergoes cycles of assembly (G0 phase) and disassembly (before mitosis). The cycles of assembly and disassembly are highly regulated, and failures in these processes can result in diseases collectively termed ciliopathies (Hildebrandt, Benzing, \& Katsanis, 2011; Reiter \& Leroux, 2017). Ciliopathies manifest as various developmental and degenerative disorders caused by mutation in a single gene. Of such disorders are polycystic kidney disease, Meckel syndrome, Joubert syndrome and Bardet-Biedl syndrome (Hildebrandt et al., 2011). Therefore, understanding cilium tasks and the mechanisms required for its assembly and disassembly are of utmost importance.

The process of assembly is well characterised (reviewed in Mirvis et al., 2018), yet the exact mechanism of ciliary disassembly is still unclear. Primary cilium disassembly occurs in two waves, the first wave occurring at the G0/G1 phase transition and a second wave prior to mitosis. The first wave of the ciliary disassembly seems to be regulated by the mitotic kinase Aurora A (Figure 1.4). The scaffolding protein NEDD9 (neural precursor cell expressed, developmentally downregulated 9, also known as HEF1) recruits Aurora A to the basal body which leads to the activation of the kinase and subsequent phosphorylation and activation of HDAC6 (histone deacetylase 6). Active HDAC6 destabilises the MTs within the cilium via

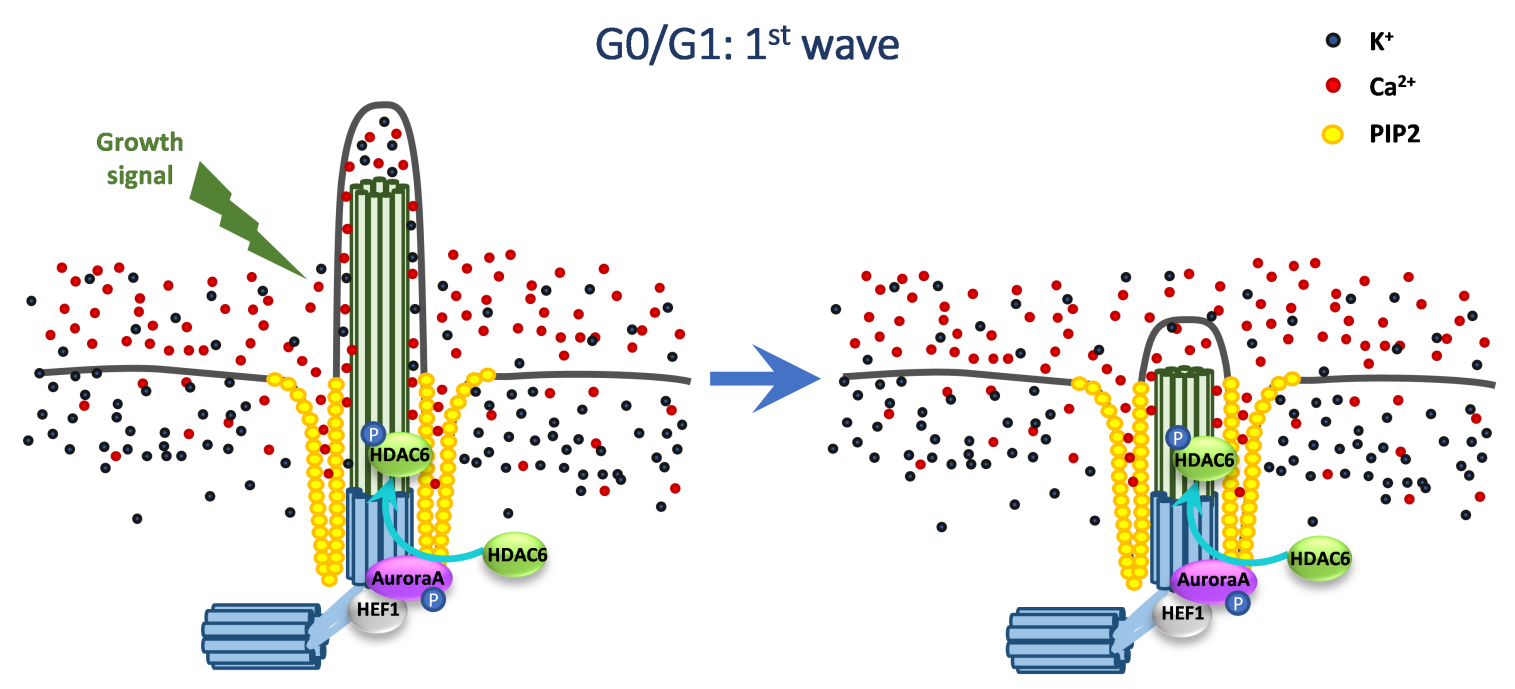

Figure 1.4: Schematic representation of the mechanism of ciliary disassembly at G0/G1 transition. Quiescent cells normally display a primary cilium, which is disassembled upon stimulation by growth factors. HEF1 (enhancer of filamentation 1) is recruited to the basal body of the cilium leading to the localisation and activation of Aurora A. Active Aurora A phosphorylates and activates HDAC6, leading to the destabilisation and depolymerisation of ciliary MTs (the centrioles are shown in blue where mother centriole is the basal body; gray line - cytoplasmic membrane; yellow circles - the ciliary pocket region enriched with PIP2 (phosphatidylinositol 4,5-bisphosphate); red and blue circles $-\mathrm{Ca}^{2+}$ and $\mathrm{K}^{+}$ions, respectively). 
deacetylation of tubulin and promotes ciliary disassembly (Pugacheva, Jablonski, Hartman, Henske, \& Golemis, 2007). At the same time, it has been shown that the first wave also includes ciliary "decapitation", driven by construction and constriction of an actin ring around the tip of the primary cilium (Phua et al., 2017).

The so-termed "second wave" of primary cilium disassembly, which occurs right before mitosis, is mechanistically similar but not identical to the first wave of the disassembly. Besides the requirement of Aurora A and NEDD9 localisation and activation at the basal body, other proteins are necessary for the process, e.g. PLK1 (Polo-like kinase 1), KIF2a (Kinesin Family Member 2A; Miyamoto et al., 2015; G. Wang et al., 2013).

After stimulation with serum during ciliary disassembly, Aurora A requires interactions with $\mathrm{Ca}^{2+}$ and CaM for full activation at the basal body (Plotnikova et al., 2012). Interestingly, we have shown that Kv10.1 localises at the centrosomes,

\section{G2/M: $2^{\text {nd }}$ wave}

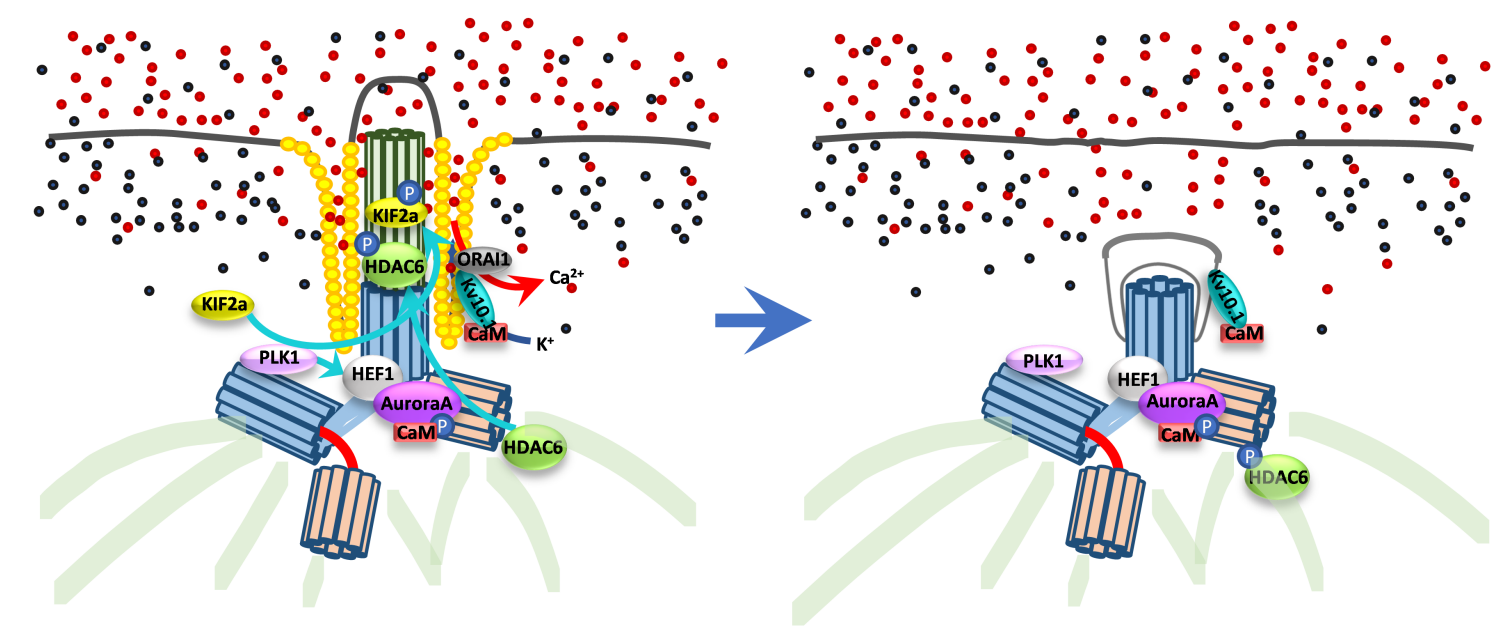

Figure 1.5: A cartoon illustrating Kv10.1 mediated resorption of the primary cilium prior to mitosis. During G2/M transition PLK1 is relocated to the basal membrane through PCM (pericentriolar matrix), leading to phosphorylation of HEF1. In turn, HEF1 scaffold protein enhances the recruitment of Aurora A at the basal body. However, for full activation Aurora A requires presence of $\mathrm{Ca}^{2+} / \mathrm{CaM}$. On the other hand, Kv10.1, which is expressed in the cells exactly at the G2/M transition, is localized at the centrosome. According to the suggested hypothesis, Kv10.1 via modulating ORAI1 activity drives $\mathrm{Ca}^{2+}$ entry necessary for Aurora A activation. Simultaneously Kv10.1 hyperpolarises the adjacent membrane resulting in the dissipation of PIP2 (phosphatidylinositol 4,5-bisphosphate) clusters. All these events thus lead to the resorption of the cilium (the growing MTs are depicted in light green; the duplicated centrioles are shown in blue (mother centriole) and orange (daughter centriole, newly synthesised); grey line - cytoplasmic membrane; yellow circles - the ciliary pocket region enriched with PIP2; red and blue circles - Ca ${ }^{2+}$ and $\mathrm{K}^{+}$ions, respectively). 
and over- or downregulation of the channel affects primary cilium disassembly (A. Sánchez, Urrego, \& Pardo, 2016a). Mutations in Kv10.1 ciliary localisation domain sequence abolished the effects both on the disassembly and Kv10.1-induced tumour growth in vivo (A. Sánchez et al., 2016a). Putting our findings in the light of current knowledge, we proposed an updated model of primary cilium disassembly (Figure 1.5). Kv10.1 activity leads to membrane hyperpolarisation locally serving a driving force for $\mathrm{Ca}^{2+}$ entry, which in turn changes the distribution of phospholipids in the cytoplasmic membrane around the base of the cilium. Therefore, both the $\mathrm{Ca}^{2+}$ ions and modulation of the lipid composition may contribute to the primary cilium disassembly (Urrego, Sánchez, Tomczak, \& Pardo, 2017).

\subsubsection{Cancer cells}

\section{Expression and regulation of Kv10.1}

The maintenance of cell number in a given tissue is achieved by an equilibrium between the cell division and the cell death. Hence, abnormalities in any of these processes can lead to the accumulation of a cell mass, a process known as tumourigenesis (Ashkenazi, Gentry, \& Jackson, 2008).

Ion channels play a critical role in cancer. In particular, potassium channels, including Kv10.1, are of the utmost interest. Potassium channels are described to be implicated in such processes as cell division, differentiation, regulation of cell volume, malignant angiogenesis, migration, metastasis and apoptosis (Figure 1.6) (C. Wang, Li, Fu, Bouras, \& Pestell, 2004).

Diverse tumours have been characterised with an altered expression of the $\mathrm{K}^{+}$ channels (Lang \& Stournaras, 2014). Particularly, ectopic expression of Kv10.1 in more than $70 \%$ of human tumours, is associated with worse prognosis (Agarwal, Griesinger, Stühmer, \& Pardo, 2010; Asher et al., 2010; Ding, Luo, Jin, Yan, \& Ai, 2007; Ding et al., 2008; Hemmerlein et al., 2006; Martínez et al., 2015; Mello de Queiroz, Suarez-Kurtz, Stühmer, \& Pardo, 2006; Ousingsawat et al., 2007; Wadhwa, Wadhwa, Dinda, \& Gupta, 2009). Moreover, Kv10.1 transfected into mammalian cells was capable of inducing a transformed phenotype, and subcutaneous implantation of the Kv10.1-expressing cells into immunodeficient mice rapidly caused invasive tumours, suggesting a role of Kv10.1 in the regulation of cell division (Pardo et al., 1999). 


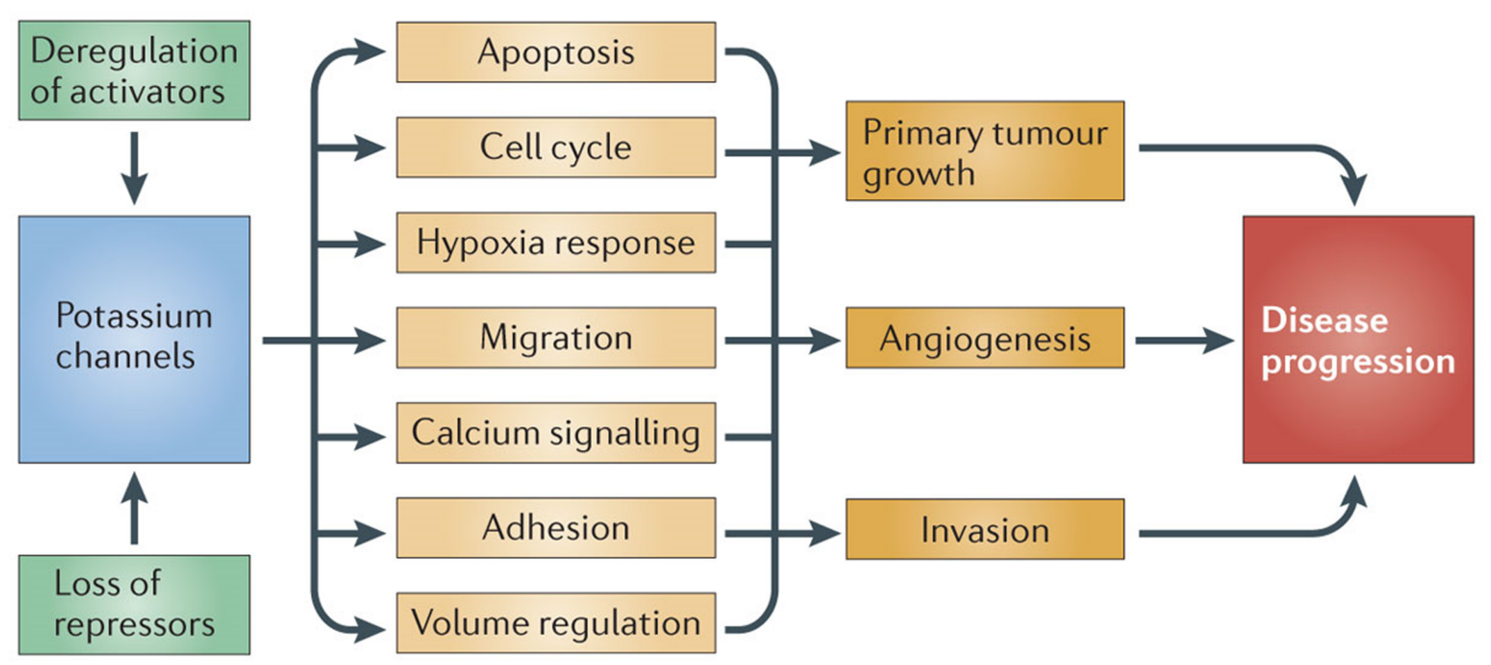

Figure 1.6: The misregulation of $\mathrm{K}^{+}$channels expression leads to tumour development and progression in multiple ways. Alterations of the expression of $K^{+}$channels, be it at the chromosomal aberrations, transcriptional, translational or post-translational levels, can substantially affect diverse cellular processes such as cell cycle, apoptosis, adhesion, migration and many others. As a result the tissue homeostasis is disrupted favouring tumour development and progression. Adapted from (Pardo 85 Stühmer, 2013).

Expression of $\mathrm{K}^{+}$channels is controlled at all levels: transcriptional, translational and post-translational (Pardo \& Stühmer, 2013). The current literature attributes the abnormal expression of Kv10.1 to the alterations in the p53-miR34-E2F1 regulatory pathway (Figure 1.7). The promoter region of $K C N H 1$ has E2F1-responsive elements, the 3' untranslated region contains miR34a-regulated sites. E2F1 increases Kv10.1 expression, whereas miR34a suppresses its expression on both transcriptional and post-transcriptional levels. miR34 is a known transcriptional target of p53, which increases expression of miR34. Therefore, p53 indirectly suppresses Kv10.1 via increase of miR34, and vice versa, loss of p53 will result in E2F1 accumulation and miR34a reduction, and subsequently in Kv10.1 aberrant expression (Lin et al., 2011). This review of events is in agreement with the findings describing Kv10.1 regulation through human papillomavirus (HPV) and oestrogens (Diaz et al., 2009): the oestrogen receptor interacts with p53 (R.-G. Liu, Wang, Song, Chen, \& Li, 2006), HPV inhibits p53 (Scheffner, Werness, Huibregtse, Levine, \& Howley, 1990) and activates E2F1 (Hwang, Lee, Kim, Seo, \& Choe, 2002). In glioblastoma drug-resistant cells, miR296-3p downregulation is concurrent with an increase in Kv10.1 expression (Bai et al., 2013). Interestingly, miR296 also interacts with p21 and downregulates the p53-p21 axis (Yoon et al., 2011). Importantly, the E2F1 transcription factor 


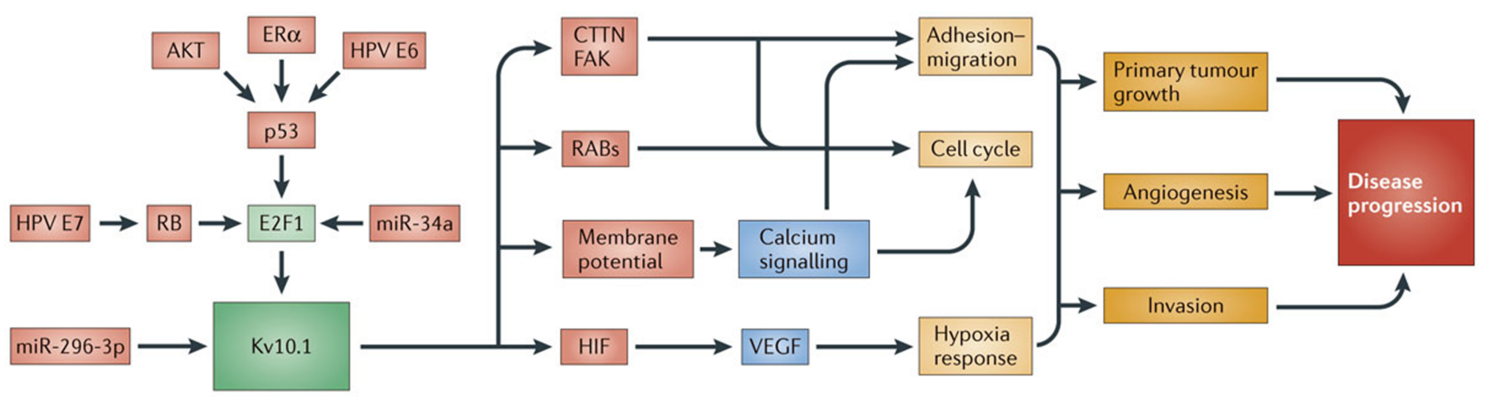

Figure 1.\%: The overview of Kv10.1 expression regulation and its implication in cancer development. The major cell cycle regulator E2F1 triggers the transcription of KCNH1 gene. The negative regulators of E2F1, like the tumour suppressor $p 53$ and $p R b$ therefore, inhibit Kv10.1 expression. Kv10.1 functionally cooperates with Rab proteins, CTTN, and so can facilitate

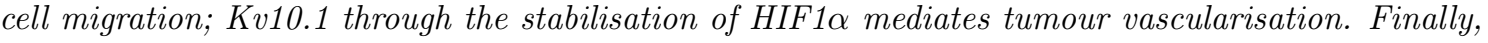
Kv10.1-mediated membrane potential changes can alter the calcium signalling and boost cell cycle. (AKT, protein kinase B; ER $\alpha$, oestrogen receptor- $\alpha$; HPV, human papillomavirus; miR, microRNA; Rab, Ras-related in brain; CTTN, cortactin; HIF1 $\alpha$, hypoxia-inducible factor $1 \alpha$; VEGF, vascular endothelial growth factor). Adapted from (Pardo \&3 Stühmer, 2013).

binds directly to KCNH1 promoter and activates the transcription (H. Lin et al., 2011; Urrego, Movsisyan, Ufartes, \& Pardo, 2016).

Genomic amplification is another mechanism of Kv10.1 overexpression (Spitzner, Ousingsawat, Scheidt, Kunzelmann, \& Schreiber, 2006), which, however, has been described only for a small proportion of cancers and alone cannot explain the recurrence of observed abnormal expression.

The expression of Kv10.1 is also a subject of an epigenetic regulation. For instance, in head and neck cancer the histone acetylation maps are altered resulting in the aberrant expression of Kv10.1 (Menéndez et al., 2012).

The expression and activity of Kv10.1 are also modulated post-translationally. In addition to the full-length form of Kv10.1, three splice variants have been identified in human brain samples. One of the splice variants is longer than the canonical protein and shows no evident differences from the normal full-length Kv10.1. In contrary, the other two splice forms, E65 and E70 are shorter and do not form a functional Kv channel since they lack all transmembrane segments. Moreover, both these shorter variants significantly inhibit the conductance of full-length channel (Ramos Gomes et al., 2015).

Human Kv10.1 conducting activity is regulated by EGFR (epidermal growth factor receptor) tyrosine kinase, which along with other protein tyrosine kinases mediates processes namely cell proliferation and differentiation, cell metabolism, 
embryogenesis, and oncogenesis (W. Wu et al., 2012). Moreover, the channel current is inhibited by an increase in intracellular $\left[\mathrm{Ca}^{2+}\right]$ (Stansfeld et al., 1996), via interaction of calcium/CaM with three CaMBDs (CaM binding domains) localised at the C- (Gonçalves \& Stühmer, 2010; Schonherr, Löber, \& Heinemann, 2000) and the N-termini of Kv10.1 (Lörinczi et al., 2016; Ziechner et al., 2006).

We have previously reported that pVHL (von Hippel-Lindau protein) promotes Kv10.1 ubiquitination (Downie et al., 2008), possibly directing the channel for proteolysis, compatible with the reduction in Kv10.1 current density upon pVHL expression and vice-versa, an increase in Kv10.1 current upon pVHL inhibition reported by others (Murata et al., 2002). pVHL is a tumour suppressor protein, mutation of which leads to von Hippel-Lindau syndrome, characterised by the occurrence of benign and malignant tumours in pancreas, adrenals, kidneys, reproductive organs and the central nervous system (reviewed in Varshney et al., 2017). pVHL functions as CRL2 (Cul2-Rbx1-Elongin B/C) E3 ubiquitin ligase substrate recognition receptor (reviewed in Cai \& Yang, 2016). Interestingly, the most studied role of CRL2-VHL complex is the degradation of the $\alpha$-subunits of HIF1 $\alpha$ (hypoxia-inducible factor 1 $\alpha$; reviewed in Q. Zhang \& Yang, 2012). It is intriguing to find out whether Kv10.1 interacts with and so modulates the activity of HIF $1 \alpha$ regulator under mild hypoxia (Downie et al., 2008).

\section{Kv10.1 in cell cycle and proliferation}

Kv channels play a crucial role in cell division, a hallmark of cancer (Hanahan \& Weinberg, 2011; Spitzner et al., 2006). The first indications that Kv10.1 is implicated in cell cycle came from the studies carried in Xenopus oocytes: spontaneous or mitosis-promoting factor induced maturation produced a dramatic reduction in the current amplitude of heterologously expressed rat Eag (Brüggemann, Stühmer, \& Pardo, 1997). Furthermore, the electrophysiological behaviour of human Kv10.1 expressed in $\mathrm{CHO}$ (Chinese hamster ovary) cells changes profoundly during the cell cycle, which can be explained by a massive rearrangement of the cytoskeleton occuring at G2/M transition (Camacho, Sánchez, Stühmer, \& Pardo, 2000). Strengthening these findings, recently we have shown that Kv10.1 is specifically expressed during late G2 and M phases (Urrego et al., 2016). 
Evidence of the importance of Kv10.1 permeation for cell proliferation comes from the observation that a small-molecule or monoclonal antibody-mediated block of the currents has an antitumour effect in breast and pancreatic cancer mouse xenograft models (Downie et al., 2008; Gomez-Varela et al., 2007). Moreover, in melanoma cells treatment with imipramine, a known inhibitor of ion channels (including Kv10.1), causes a significant decrease in DNA synthesis and cell proliferation rates without considerable effects on apoptosis (Gavrilova-Ruch et al., 2002).

Further proofs of the role of Kv10.1 in cell proliferation are obtained from siRNA (small interfering RNA) transfection studies. For instance, downregulation of $K C N H 1$ decelerates cell proliferation in several human tumour cell lines (Weber et al., 2006), while its activation in human melanoma cells by arachidonic acid enhances the proliferation (Gavrilova-Ruch, Schönherr, \& Heinemann, 2007). Spitzner et al. have shown that spontaneous transformation of colon carcinoma T84 cells is accompanied by an increase in the proliferation rate and amount of Kv10.1 and Best1 (bestrophin 1, $\mathrm{Ca}^{2+}$-activated Cl- channel); silencing of Kv10.1 and Best1 reduces proliferation of the transformed cells suggestively by regulating calcium signalling and cell volume (Spitzner et al., 2008).

\section{Kv10.1 in cell migration}

Migration and invasion are the prerequisites of metastasis, a critical event in cancer progression. During these processes, tumour cells detach, move and adhere at a new location (B. Martin et al., 2013). Potassium channels are implicated in every step of these processes (Becchetti \& Arcangeli, 2010; Schwab, Fabian, Hanley, \& Stock, 2012), which they achieve in at least three ways: modulating the cell volume, membrane potential and interacting with migration complex (Ouadid-Ahidouch, Ahidouch, \& Pardo, 2016).

Inhibition of Kv10.1 has been shown to diminish the migration of leukaemia (Agarwal et al., 2010) and breast cancer cells (Hammadi et al., 2012) in vitro. The underlying mechanism is suggested to be through the increase of $\mathrm{K}^{+}$permeability, which in turn hyperpolarises the cytoplasmic membrane and, thus, facilitates ORAI1 (calcium release-activated calcium channel protein 1 channel)-driven calcium entry Agarwal et al., 2010; Hammadi et al., 2012; Pardo \& Stühmer, 2013). 
Kv10.1 may also influence cell migration, possibly, by rearranging the cytoskeleton. Interestingly, Kv10.1 electrophysiological properties are modulated by interactions with cytoskeletal components (Camacho et al., 2000). As shown in MCF-7 cells, Kv10.1 colocalises with FAK (focal adhesion kinase) at focal adhesion, which is driven by the interaction with cortactin (Herrmann, Ninkovic, Kohl, Lörinczi, \& Pardo, 2012). However, the interaction with cytoskeleton regulatory molecules is better characterised for another member of the EAG family, hERG1 (human EAG related gene 1). hERG1 is suggested to promote cell migration via interaction with ß1-integrins and FAK proteins (Arcangeli et al., 2004; Cherubini et al., 2005, 2002).

\section{Kv10.1 in angiogenesis}

Angiogenesis is the process of new blood vessels genesis from pre-existing ones (Ronca, Benkheil, Mitola, Struyf, \& Liekens, 2017). Early in 1971, Sherwood et al. proposed the idea of viewing a solid tumour as an ecosystem: the cancer cell creates favourable conditions for endothelial cells to divide, thus promoting neovascularisation, needed for the cancer cells to grow (Sherwood, Parris, \& Folkman, 1971).

Kv10.1 is also implicated in angiogenesis. A strong positive correlation has been found between Kv10.1 and HIF1 $\alpha$ (Lai et al., 2014). Upon expression of Kv10.1, even a subtle drop in the partial pressure of oxygen activates HIF $1 \alpha$, leading to the activation of VEGF (vascular endothelial growth factor among many other downstream targets Downie et al., 2008), and, consequently, neovascularisation (Ferrara \& Davis-Smyth, 1997; Koong et al., 2000; Semenza, 2013; Takahashi, Kitadai, Bucana, Cleary, \& Ellis, 1995). Consistently, Kv10.1-positive tumour cells display significantly increased secretion of VEGF and vascularisation (Downie et al., 2008). Thus, expression of Kv10.1 can be seen as an advantage for tumours making them more resistant to hypoxia. However, resistance to hypoxia seems not to be the only benefit for the cancer cells expressing the channel, as Kv10.1 promotes tumour progression under conditions independent of hypoxia like in leukaemic cells (Agarwal et al., 2010). 


\section{Kv10.1 in apoptosis}

Cell death is a tightly regulated process, of which several forms have been described. One of the most prominent ways is apoptosis, the programmed cell death. Yet cancer cells developed mechanisms of escaping the cell death tilting the balance towards "overcrowding" the tissue. Therefore, developing these evading mechanisms is the game-changing factor critical for cancer treatment (Bortner \& Cidlowski, 2014; Tan, Tan, \& Muhammad, 2015).

The voltage-gated delayed rectifier potassium channels among many others are involved in the induction of apoptosis (Bortner \& Cidlowski, 2014; Lang et al., 2007). One of the characteristics of apoptosis is a loss of cell volume, also known as cell shrinkage (Bortner \& Cidlowski, 2007, 2014) and termed AVD (apoptotic volume decrease; Maeno, Ishizaki, Kanaseki, Hazama, \& Okada, 2000). The changes in intracellular ionic composition, in particular loss of potassium ions, is the cornerstone of AVD and subsequent triggering of the apoptotic mechanism (Bortner \& Cidlowski, 2014; Cain, Langlais, Sun, Brown, \& Cohen, 2001; Thompson, Langlais, Cain, Conley, \& Cohen, 2001). Although the exact mechanism of AVD driven by potassium depletion is not entirely understood, direct block of potassium channels is shown to protect cells from apoptosis (X. Wang, Xiao, Ichinose, \& Yu, 2000).

However, the role of $\mathrm{Kv}$ channels in the regulation of apoptosis is contradictory. For instance, the voltage-gated potassium channel Kv1.3 is directly involved in apoptosis. It has been demonstrated that inhibition of Kv1.3, which is localised at the inner mitochondrial membrane, through interaction with BAX (Bcl2-associated $\mathrm{X}$ protein) or through selective inhibitors of Kv1.3 leads to hyperpolarisation of the membrane, followed by rise in reactive oxygen species and, subsequently, cytochrome c release (Leanza et al., 2012, 2017, 2015; Szabó et al., 2008). Growth arrest and apoptosis have been observed in osteosarcoma cells treated with CRAd5.TRAIL/siEAG1 adenoviral vector which simultaneously downregulates Kv10.1 and overexpresses TRAIL (TNF-related apoptosis inducing ligand; X. Wu et al., 2013).

The tumour-specific expression of Kv10.1, meaning its low abundance in the peripheral tissue, makes it a promising indexing tool for anticancer drug delivery. For example, a single-chain antibody raised against Kv10.1 (scFv62) and fused with human soluble TRAIL induces programmed cell death in sensitised Kv10.1-positive 
prostate cancer cells while sparing the healthy ones (Hartung \& Pardo, 2016; Hartung, Stühmer, \& Pardo, 2011).

\subsection{Cell cycle: stages and checkpoints}

\subsubsection{Overview of the cell cycle}

During cell division, highly coordinated series of events are completed that are collectively termed the cell cycle (van den Heuvel, 2005). The cell cycle consists of four phases: S (Synthesis) and M (mitotic; mitosis and cytokinesis) phases which are separated by so-called G (Gap) phases, G1 and G2 (Figure 1.8; G. M. Cooper, 2000).

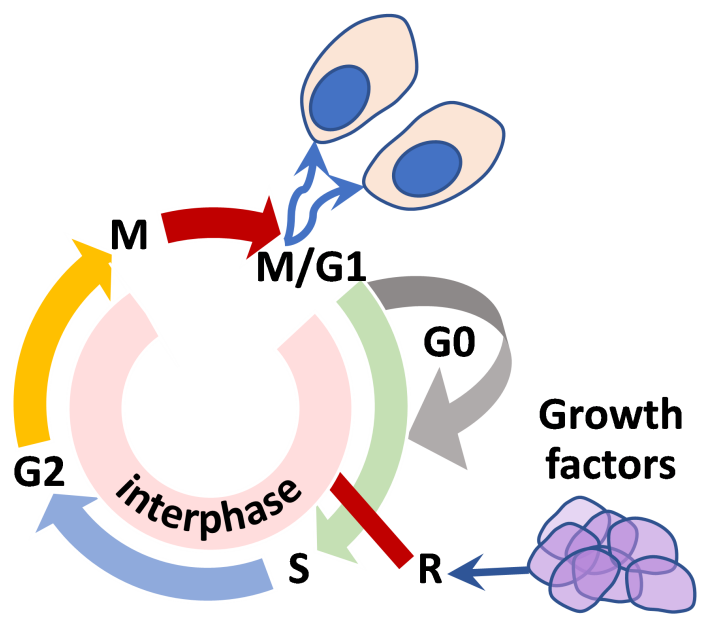

Figure 1.8: Simplified representation of the cell cycle. The cell cycle in eukaryotes consists of four discrete phases: $M, G 1, S$, and G2. Cells grow throughout interphase, which includes G1, $S$ and G2. DNA replication takes place in $S$ phase, and sister chromatid segregation and cytokinesis - during $M$ phase. The availability of growth factors is the decision-maker for a cell to commit into a new cycle. This point is located in late G1 and is known as the "Restriction point". If the cell is deprived of growth factors during G1, the cell leaves the cycle and enters a quiescent state termed G0. The relative length of each phase shown in the diagram is common for a rapidly dividing mammalian cell.
The four phases G0, G1, S and G2 correspond to the morphologic "interphase". During interphase, a series of changes take place both in a newly formed cell and its nucleus before it again becomes capable of the division. It is also known as the preparatory phase or intermitosis. S phase consists of an accurate duplication of the genome. During G1 phase cells transition from the completion of $\mathrm{M}$ phase to the $\mathrm{S}$ phase of the next cycle. In G2 phase, which is between S and M phases, cells complete the final preparatory steps necessary for the M-phase cells (G. M. Cooper, 2000).

Mitosis (nuclear division) is the most dramatic stage of the cell cycle, corresponding to the separation of sister chromatids and formation of genetically iden-

tical two daughter cells (Figure 1.9). The M phase consists of the following steps:

- prophase - chromatin condensation takes place; the centrosomes are separated towards the opposite poles of the nucleus by the action of kinesins, such as 
Eg5; in late prophase the nuclear envelope breakdown takes place (Agircan, Schiebel, \& Mardin, 2014; Dekker, 2014; Schlaitz, 2014);

- metaphase - alignment of sister chromatids along the metaphase plate; mitotic spindle assembly checkpoint (Bastians, 2015; Holland \& Cleveland, 2009);

- anaphase - the cohesin ring is opened, chromatid cohesion is lost, and by pulling forces of the spindle the sister chromatids are driven to opposite sides of the cell (Lara-Gonzalez, Westhorpe, \& Taylor, 2012);

- telophase - reformation of the nuclear envelope; decondensation of chromosomes; reappearance of nucleoli (Larijani \& Poccia, 2009).

After completion of mitosis, roughly equal shares of the cytoplasm, organelles, cell membrane and, normally, equal number of chromosomes are distributed between newly forming two daughter cells. This process is known as cytokinesis (D'Avino, Giansanti, \& Petronczki, 2015), and together with mitosis they de-

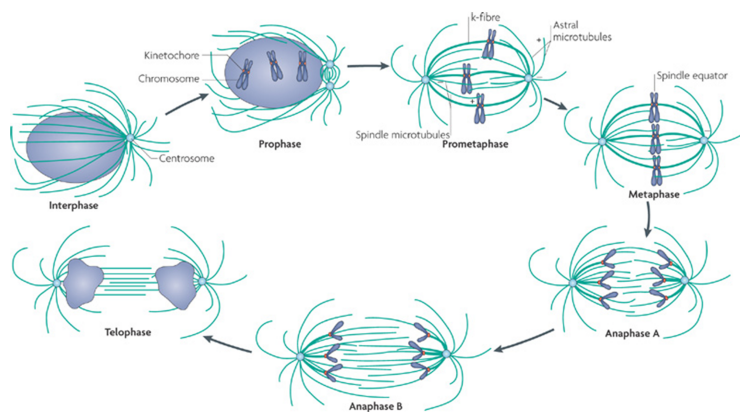

Figure 1.9: Simplified view of different stages of mitosis. fine the M phase, which lasts only about an hour. Therefore, approximately $95 \%$ of the time a cell spends in interphase (G. M. Cooper, 2000).

Besides the described Gap phases, environmental and developmental cues can drive the G1 cells into a quiescent, non-cycling state termed G0 (Figure 1.8; G. M. Cooper, 2000). Normally, external triggers, such as availability of growth factors, will define whether a cell commits to a new cycle of division or exits it. Time-wise, this decision-making point is located in late G1 and is known as the "Restriction point" (Pardee, 1974). If the cell is deprived of growth factors during G1 phase, the cell will then exit into G0 phase. However, if there are sufficient growth factors available, then the cell passes the Restricition point and continues cycling. From here on, the progression through each following phase is no longer dependent on the extrinsic factors, but rather is orchestrated intrinsically by the cell cycle machinery (van den Heuvel, 2005). 
The normal propagation of each phase of cell cycle, especially the transitions from one phase into another, is regulated by mechanisms termed "checkpoints". A checkpoint will halt a cell in a given phase until the condition of passing the particular checkpoint is met (Kastan \& Bartek, 2004). Due to the complexity of cell cycle, in the following sections, I will describe only the major events happening at a given phase and mostly focus on the checkpoint mechanisms which ensure the errorless propagation of the cell cycle.

\subsubsection{Regulation of the G1/S transition: $\mathrm{pRb}-\mathrm{E} 2 \mathrm{~F}$ axis}

As already mentioned, the decision of committing to a cell cycle is contingent on the availability of growth factors. Upon mitogen cues, several signalling pathways that regulate cyclin D1 expression are activated (C. Wang et al., 2004). For instance, activation of the Ras-MAPK pathway through tyrosine kinase receptors or integrins leads to cyclin D1 accumulation (Poon, 2016), which is crucial for the activation of the pRb-E2F1 axis (Figure 1.10).

The pRb (retinoblastoma protein) E2F1 pathway regulates the transcription of the genes necessary for G1/S transition, the Restriction point (Pardee, 1974). In the absence of mitogens, pRb

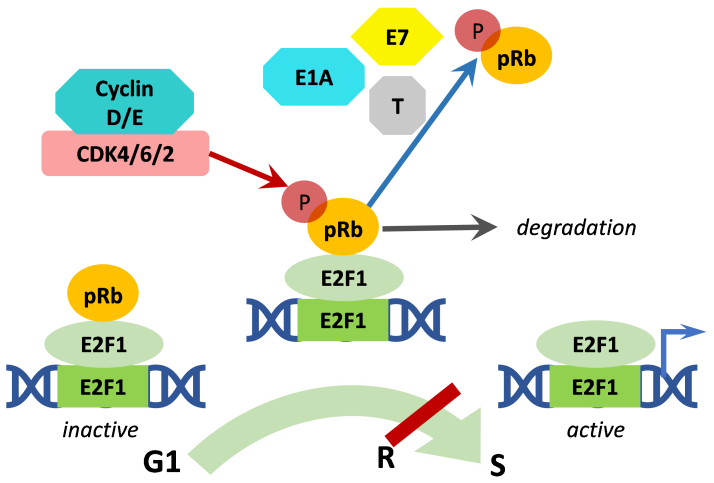

Figure 1.10: Mechanism of G1/S transition. Upon stimulation with growth factors the expression of cyclin $D$ and $E$ increases, which results in complexing of cyclin $D(E)$ with and activating CDK4(6). This complex phosphorylates and targets $\mathrm{p} R \mathrm{~b}$ for degradation, thus releasing the E2F1 factor from the inhibitory complex and activating the transcription of E2F1-dependent genes. Several viral oncogenes have been described to sequester $p R b$ from the E2F1 complex and thereby facilitate the G1/S transition ("P" in green circles and the blue arrows - activating phosphorylation; " $P$ " in red circles and the red arrows - inactivating phosphorylation; the grey arrow - proteolysis; the red line indicates the restriction point, " $R$ ").

directly binds to the transactivation do-

main of E2F1 and the promoter regions of the genes necessary for G1/S transition, thus repressing gene transcription (Adams \& Kaelin, 1995; Macaluso, Montanari, \& Giordano, 2006). Progression through the Restriction point, therefore, depends on $\mathrm{pRb}$ inhibition.

$\mathrm{pRb}$ phosphorylation is a cell cycle-dependent phenomenon and leads to its inac- 
tivation. In early G1 phase, mitogens induce rapid expression of cyclin D1 (Hitomi \& Stacey, 1999), which couples with the kinases CDK (cyclin-dependent kinase) 4 or CDK6. Cyclin D1/CDK4(6) complexes phosphorylate and inactivate pRb, consequently leading to conformational changes and release of the E2F1 transcription factor from the inhibitory complex (Dowdy et al., 1993). Released and activated E2F1 transcription factor, then, drives the expression of an array of genes, including cyclin E1. Expression of cyclin E1 is periodic and reaches its maximum during the transition from G1 to S phase (Ohtsubo, Theodoras, Schumacher, Roberts, \& Pagano, 1995). In the middle to late G1 phase, cyclin E1 pairs with CDK2 forming an active complex, which in turn phosphorylates $\mathrm{pRb}$. Therefore, the concentration of liberated E2F1 increases, leading to a further accumulation of cyclin E1. Moreover, E2F1 activity is modulated by cyclin E1/CDK2-mediated phosphorylation. This constitutes a classical feedback mechanism: cyclin E1 stimulates its transcription (Möröy \& Geisen, 2004).

Some DNA viruses often found in certain tumours can also modulate pRb activity. For example, in tumour cells, pRB/E2F1 interaction is broken by binding of tumour virus oncoproteins, such as HPV E7, adenoviral E1A to pRb, and, thus, activating E2F1 (Moran, 1993).

\subsubsection{The DNA damage-induced checkpoint}

DNA replication is a complex process, and despite the high fidelity of it some errors still occur, making a mechanism to control and correct the errors indispensable. Such mechanism is the DNA damage-activated checkpoint, which recruits the DDR (DNA damage response) machinery in order to spare time for the cell to repair the lesions and then resume the cell cycle progression (Shaltiel, Krenning, Bruinsma, \& Medema, 2015).

DDR is a kinase cascade, which detects (sensor proteins), signals (mediators) and causes cell cycle arrest (effectors) to ensure that DNA lesions are repaired (J. W. Harper \& Elledge, 2007). The type of DNA lesion dictates which arm of DDR should be activated. Double-strand breaks (DSBs) activate ATM (ataxia telangiectasia mutated kinase), which preferentially phosphorylates CHK2 (checkpoint kinase; J.-H. Lee \& Paull, 2005). Single-strand breaks (SSBs) activate ATR (ATM and Rad3 
related kinase), which targets and activates CHK1 (Zou \& Elledge, 2003).

The recent findings show that DDR and cell cycle machinery are intertwined, meaning that depending on the cell cycle phase one or the other arm of DDR will be activated independent of the type of DNA lesions. This allows to choose the target proteins of the DDR in a cell cycle phase-specific manner (Shaltiel et al., 2015).

\section{G1 phase DNA damage-activated checkpoint}

The major DNA damage-activated checkpoint in cells passing through G1 phase is the ATM(ATR)-CHK2(CHK1)-p53/MDM2-p21 pathway. This checkpoint induces prolonged or even permanent arrest in G1 phase (Figure 1.11; Chen \& Poon, 2008). The expression of ATM and CHK2 is relatively constant, whereas a moderate periodicity is observed in case of ATR and CHK1. Their expression gradually increases during G1 phase and reaches higher levels closer to the G1/S transition phase, where the kinetic activity is more needed (Gately, Hittle, Chan, \& Yen, 1998; Lukas et al., 2001). ATM and ATR, along with CHK1 and CHK2 phosphorylate the p53 transcription factor and activate it (Awasthi, Foiani, \& Kumar, 2015; Bartek \& Lukas, 2003; Kastan \& Lim, 2000). Additionally, the ubiquitin ligase MDM2 (mouse double minute 2 homolog), one of the major negative regulators of p53,

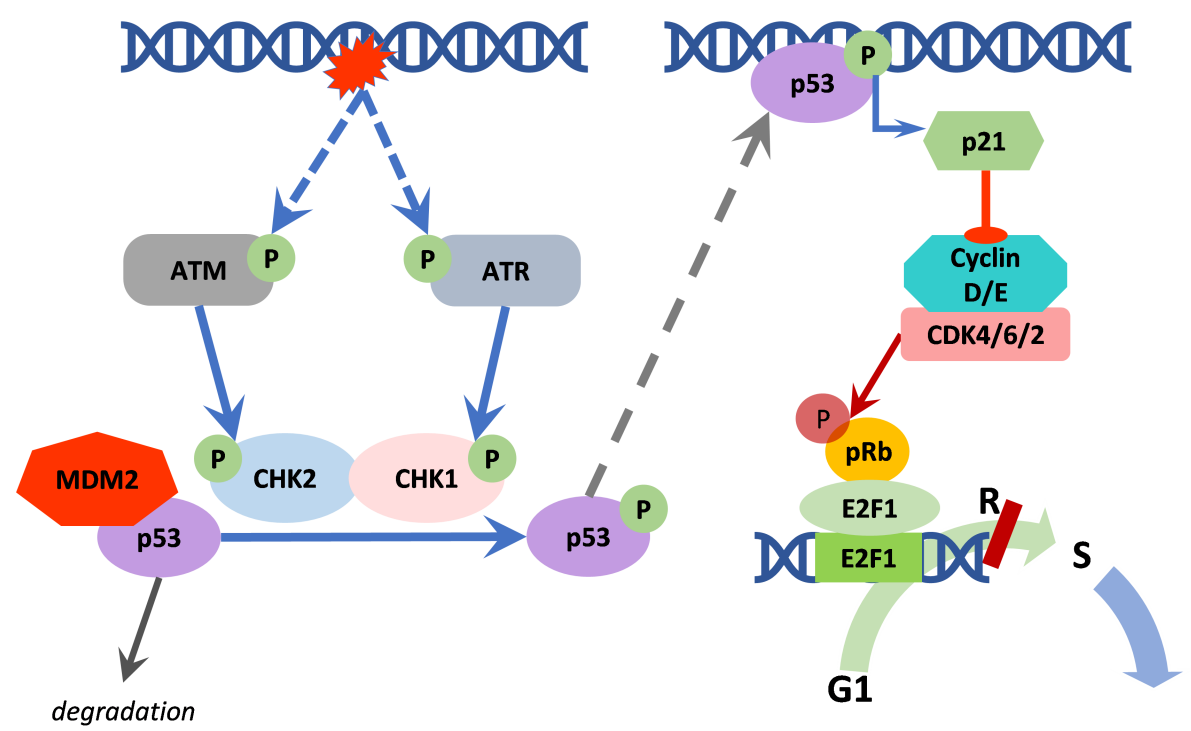

Figure 1.11: Mechanism of DNA damage checkpoint activation and cell cycle arrest in G1 phase. The detailed description is given in the main text; "P" in green circles and the blue arrows - activating phosphorylation; " $P$ " in red circles and the red arrows - inactivating phosphorylation; the dashed arrow in grey - translocation; the red arrow with flat end - inhibition; the dashed arrow in blue - indirectly activating phosphorylation. 
is inactivated by ATM, ATR, CHK2 and CHK1. Therefore, the activating and inactivating phosphorylations of p53 and MDM2, respectively, result in the sustained increase of active p53. In this context, the crucial target of p53 is the p21. The p21 inhibits the cyclin E1/CDK2 complex, the driver of the G1/S transition, and thereby causes an arrest in G1 phase. As mentioned above, this complex is required for the activation of E2F1 and, subsequently, initialisation of DNA synthesis. In summary, the G1 DNA damage-activated checkpoint actuates the two pivotal tumour suppressor pathways controlled by p53 and pRb (Kastan \& Bartek, 2004).

\section{Intra-S phase DNA damage-activated checkpoint}

Once the restriction point is passed, the cell progresses into the $\mathrm{S}$ phase, where the DNA replication and centrosomal duplication take place (Nigg \& Stearns, 2011). These events are tightly coordinated to eventuate only once per cell cycle (Huang \& Zhang, 2011; Sclafani \& Holzen, 2007). Although these two processes occur in distinct compartments of the cell and are spatially separated by the nuclear membrane, yet they use the same licensing proteins and are subject to the same regulators such as cyclinE1/CDK2 and cyclinA2/CDK2 (Ferguson \& Maller, 2010; Huang \& Zhang, 2011; Jackman, Kubota, den Elzen, Hagting, \& Pines, 2002).

Cyclin A2 starts accumulating at the

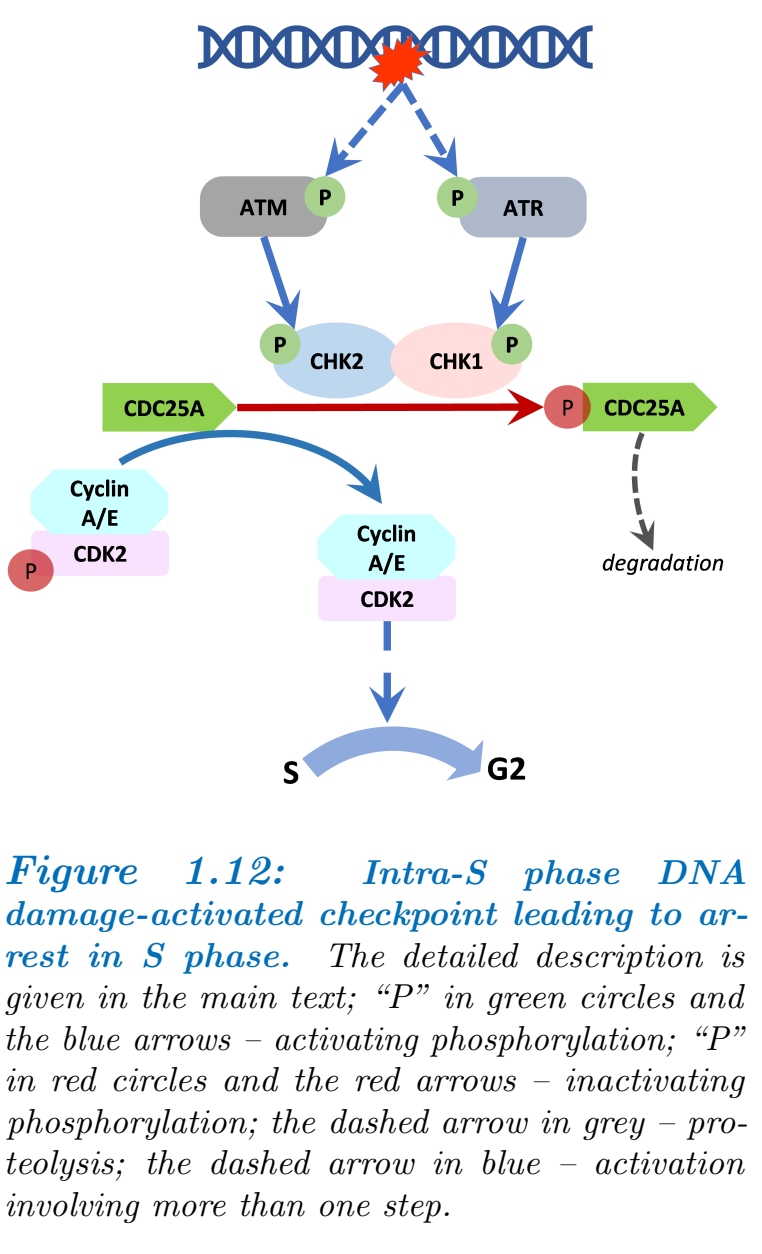
G1/S transition and reaches its utmost level during the S phase (Henglein, Chenivesse, Wang, Eick, \& Bréchot, 1994). At the beginning of S phase, it complexes with CDK2, which is believed to finish the work of cyclin E1/CDK2: propagation of DNA synthesis from already assembled replication complexes, and hindering the formation of new 
ones. Therefore, cyclin A2/CDK2 complex prevents DNA re-synthesis. Together, cyclin E1 and A2 start and guide, respectively, the DNA replication (Coverley, Laman, \& Laskey, 2002).

If the DNA replication has already started, but some DNA lesions persist, the replication fork will stall at the damage site. The latter will turn the DDR machinery on, preferentially through ATR, slowing the S phase progression (Figure 1.12; Awasthi et al., 2015). Active ATM and ATR will phosphorylate CHK2 and CHK1, respectively, as described earlier. However, during S phase, CHK1 and CHK2 phosphorylate and so inactivate the phosphatase CDC25A (cell division cycle; Sørensen et al., 2003). CDC25A is necessary for actuating the cyclin A2/CDK2 and cyclin E1/CDK2 complexes (Donzelli \& Draetta, 2003; Girard, Strausfeld, Fernandez, \& Lamb, 1991; Nyberg, Michelson, Putnam, \& Weinert, 2002). Thereby, activated intra-S phase checkpoint will slow down the replication until the DNA lesion is repaired.

\section{G2 phase DNA damage-activated checkpoint}

The propagation from G2 to M phase is accompanied by dramatic changes in cell architecture: NEB (nuclear envelope breakdown), chromosome condensation and bipolar mitotic spindle assembly. The effector kinase in this stage of the cell cycle is CDK1 in a complex with either cyclin A2 or cyclin B1. Cyclin B1/CDK1 complex, also termed MPF (mitosis-promoting factor) is considered to be the driving force of $\mathrm{G} 2 / \mathrm{M}$ transition. The translocation of this complex into the nucleus is driven by cyclin A2/CDK1 complex; shortly after that, the NEB takes place (Gong et al., 2007).

CDK1 activation requires a series of phosphorylation and dephosphorylation events and complex formation with mitotic cyclins A and B (Figure 1.13). Cyclins A2 and B1 have an oscillatory pattern in expression. As already mentioned, cyclin A2 accumulates starting from $\mathrm{S}$ phase until early mitosis, whereas cyclin B1 is expressed in late G2 and is a subject of ubiquitin-mediated proteolysis during conversion from metaphase to anaphase. CDK1 is primarily inactive; upon binding to cyclin B1, the kinase is phosphorylated mainly by WEE1 at Tyr15 and thus maintained inactive (Ma \& Poon, 2011). 


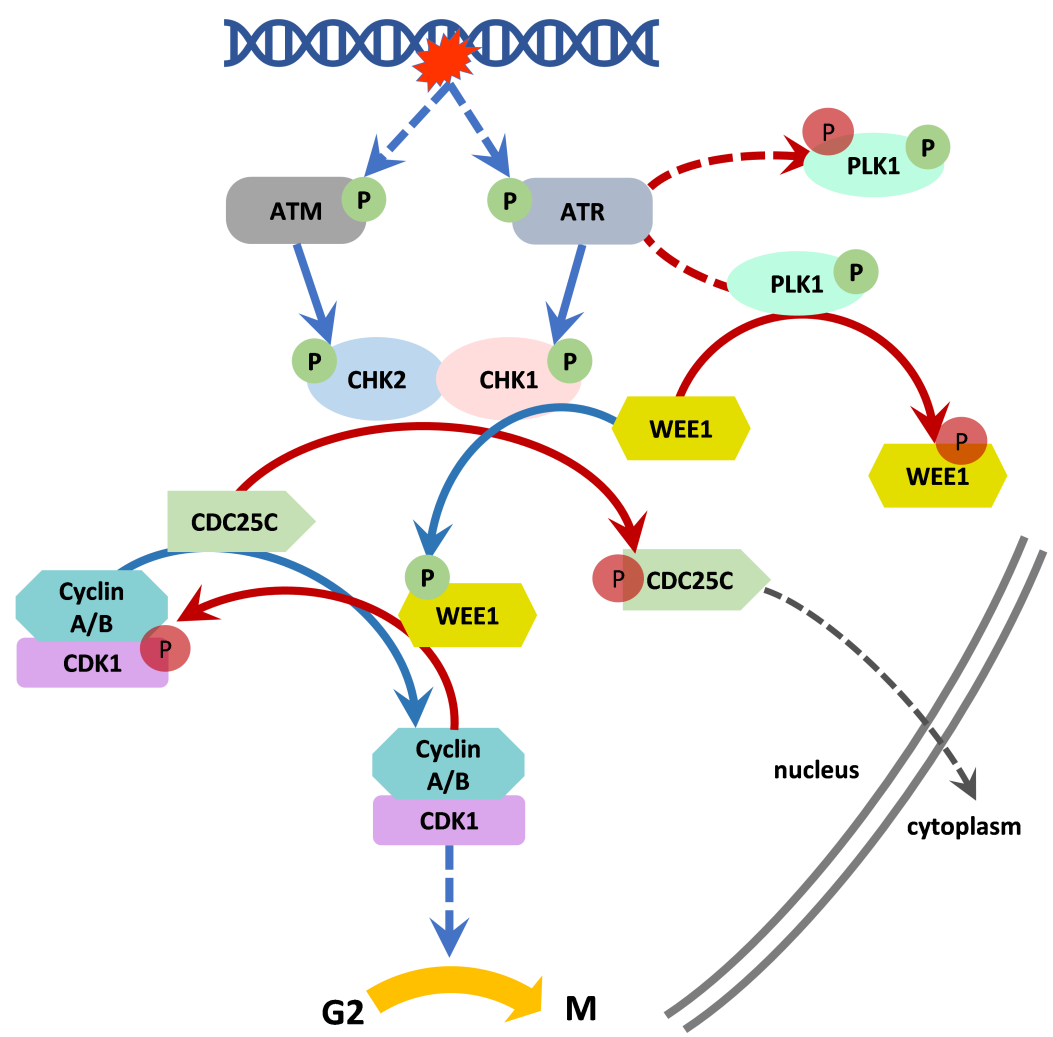

Figure 1.13: Simplified mechanism of G2 arrest caused by activation of DNA damage-induced checkpoint. The detailed description is given in the main text; "P" in green circles and the blue arrows - activating phosphorylation; "P" in red circles and the red arrows inactivating phosphorylation; the dashed arrow in grey - translocation; the dashed arrow in blue or red-activation or inactivation, respectively, involving more than one step.

The actuation of cyclin B1/CDK1 is triggered by cyclin A2/CDK1 complex. Cyclin A2/CDK1 has been shown to activate Bora (aurora borealis), a cofactor necessary for Aurora A activation (Vigneron et al., 2018). One of the multiple substrates of this kinase is PLK1 (Polo-like kinase 1; Macůrek et al., 2008; Seki, Coppinger, Jang, Yates, \& Fang, 2008), which targets and activates CDC25C phosphatase (Roshak et al., 2000). CDC25C is responsible for removing the inhibitory phosphorylation at Tyr15 on the cyclin B1/CDK1 complex (Lindqvist, Rodríguez-Bravo, \& Medema, 2009). Besides, PLK1 phosphorylates and inhibits the WEE1 kinase (Watanabe et al., 2005). Moreover, the now fully active cyclin B1/CDK1 complex phosphorylates both WEE1 and CDC25C leading to their inactivation and activation, respectively (Perry \& Kornbluth, 2007). Thereby, the activation of cyclin B1/CDK1 complex represents an example of a positive feedback loop (Poon, 2016).

Despite the careful control during G1 and S phases, some DNA lesions can still remain, or new ones may occur. Therefore, DDR is also activated during the 
G2 phase (Figure 1.13). Cyclin B1/CDK1 complex is the decisive target of the DNA damage activated checkpoint during G2 phase. As before, the same kinase cascade is activated. However in this case the effector kinases CHK1 and CHK2 inhibit CDC25C and activate WEE1 via phosphorylation. This leads to tilting the equilibrium towards the inactive cyclin B1/CDK1. Also, DDR has been shown to inactivate PLK1 (Bruinsma et al., 2017; Bruinsma, Raaijmakers, \& Medema, 2012; Smits et al., 2000). Hence, due to the activated DNA damage pathway the complexes cyclin A2/CDK1 and cyclin B1/CDK1 will not function preventing the premature start of the mitosis and arresting the cells in G2/M phase.

In summary, the DNA damage-activated checkpoint retards the cell cycle progression at different phases giving the cell sufficient time to eliminate all DNA lesions and, thus, ensures genomic stability.

\subsubsection{The mitotic spindle assembly checkpoint}

The closing phase of the cell cycle, the $\mathrm{M}$ phase is the most critical stage, which ensures correct distribution of genetic information between the newly forming daughter cells. The unresolved errors during this phase can generate genomic instability, which underlies the "hallmarks" of cancer (Hanahan \& Weinberg, 2011).

The process of chromosomal segregation is orchestrated by an intertwined network of kinases, phosphatases, ubiquitin ligases and cytoskeleton components, and requires rearrangement of the entire cell architecture. That includes NEB, remodelling of the actin skeleton and endoplasmic reticulum, fragmentation and dispersing of the Golgi apparatus, fission of mitochondria, condensation of chromosomal DNA and bipolar mitotic spindle formation (Jongsma, Berlin, \& Neefjes, 2015).

Despite this complexity, the entire process can be narrowed down to one primary regulative mechanism, which is known as SAC (spindle assembly checkpoint). The aim of SAC is to ensure biorientation of chromosomes at the metaphase plate by modulating the kinetochore-MT attachments. Once this condition is met, the checkpoint is turned off and the chromosome movement to opposite poles is initiated (Musacchio \& Salmon, 2007).

The formation of bioriented chromosomes at the metaphase plate is achieved by an error-correction process coined "search and capture" (Heald \& Khodjakov, 2015; 


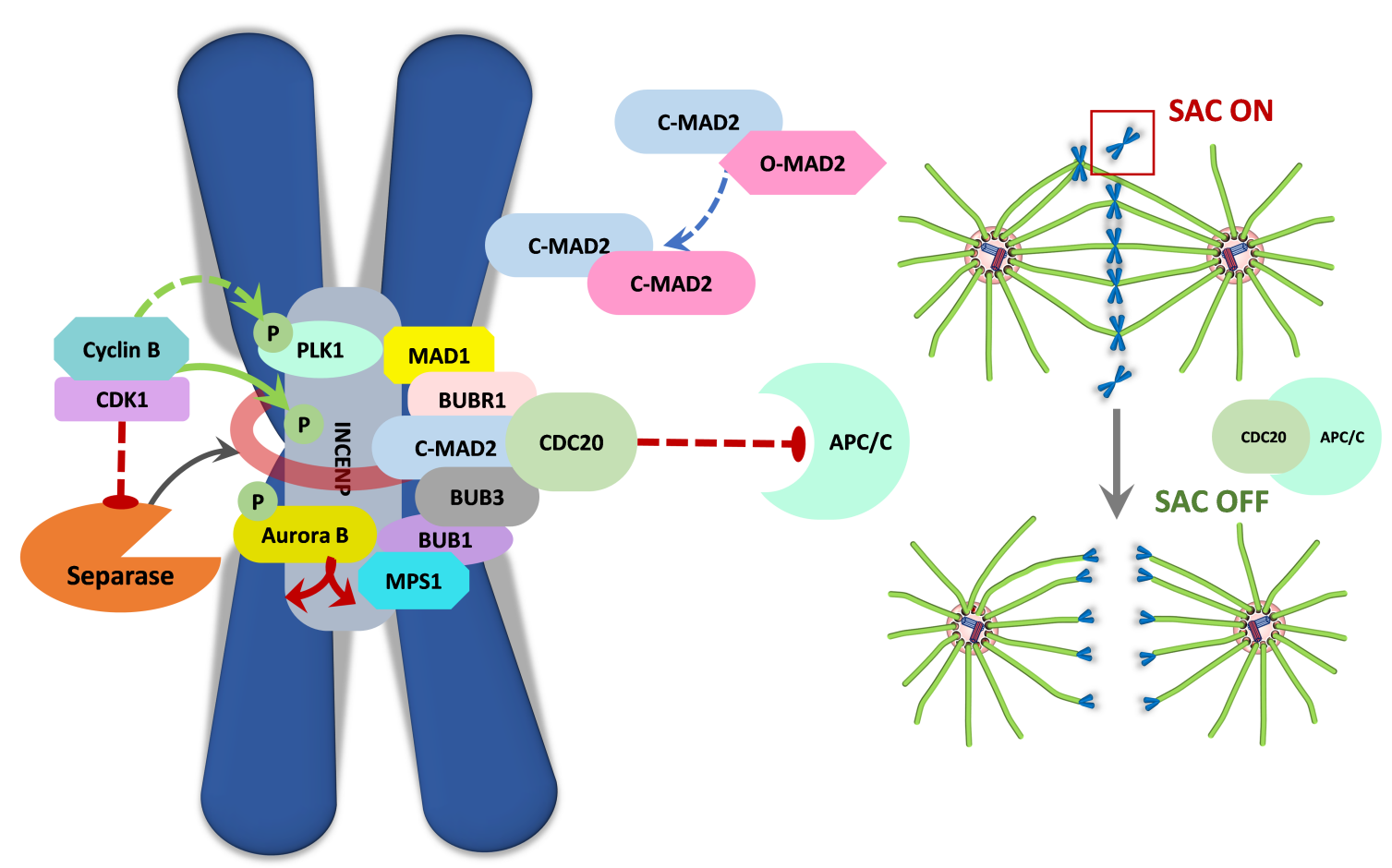

Figure 1.14: Mechanism of action of spindle assembly checkpoint. The detailed description of the cartoon is given in the main text. "P" in green circles and the green arrows activating phosphorylation; the red arrows - inactivating phosphorylation; the dashed arrow in redinhibition involving more than one step; the arrow in grey - cleaving activity of separase; the dashed arrow in blue - conformational change leading to activation of MAD2; the semi-transparent red circle - cohesin.

Kirschner \& Mitchison, 1986; Mazia, 1987). Several kinases are relocated to the kinetochores - a protein complex assembled on a centromere region of each chromatid which ensures attachment of MTs (Cleveland, Mao, \& Sullivan, 2003). Some of these proteins are CDK1, PLK1 and the CPC (chromosomal passenger complex). The CPC is comprised of Aurora B, Borealin, INCENP (inner centromere protein) and Survivin, which also controls later stages of M phase, i.e. cytokinesis (Poon, 2016).

These proteins together ensure that each chromosome is connected to the MTs emerging from the opposite poles and the force applied by the MTs on them is equal from both sides creating tension between the two sister chromatids. If no tension is achieved, then the kinases present here, mainly Aurora B, will be capable of reaching the assembling tips of MTs and destabilising the kinetochore-MT attachments by phosphorylation. The process of MTs catching the chromosomes will be continued until the tension is achieved, leading to the spatial inhibition of Aurora B and other proteins involved in the regulation of kinetochore-MT attachments (Krenn \& Musacchio, 2015). 
The chromosome attach to and align at the equatorial region of the mitotic spindle stochastically, that is some chromosomes are faster than others. Hence, anaphase should be prevented until every single chromosome is correctly positioned. This check is carried out by SAC (Lampson, Renduchitala, Khodjakov, \& Kapoor, 2004; Musacchio \& Salmon, 2007).

The central effector of SAC is a diffusible complex called the MCC (mitotic checkpoint complex, Figure 1.14; Poon, 2016). SAC contains the APC/C (anaphase promoting complex/cyclosome) activator CDC20 and the proteins MAD2L1 (mitotic arrest deficient 2 like 1, also termed MAD2), BUBR1 (BUB1-related protein 1, also known as MAD3) and BUB3 (budding uninhibited by benzimidazoles 3 homolog; Musacchio \& Salmon, 2007). These proteins are recruited explicitly to kinetochores lacking MT attachments (Moyle et al., 2014; Skoufias, Andreassen, Lacroix, Wilson, \& Margolis, 2001; Waters, Chen, Murray, \& Salmon, 1998). MCC sequesters CDC20 by binding to it with MAD2, which leads to transitioning of MAD2 from an open less stable (O-MAD2) to the closed stable conformation (C-MAD2; Poon, 2016). It is believed that C-MAD2 acts like a prion and converts more O-MAD2 to C-MAD2 without further need of MCC (Mapelli, Massimiliano, Santaguida, \& Musacchio, 2007).

Most importantly, the SAC ensures maintenance of $\mathrm{APC} / \mathrm{C}$ in the inactive state by sequestering its substrate recognition subunit and activator CDC20. Therefore, the polyubiquitylation and degradation of two key substrates, cyclin B1 and securin is prevented (Musacchio \& Salmon, 2007). The importance of keeping cyclin B1/CDK1 complex active has already been discussed above.

The sister chromatids are held together by cohesin, which, in order to license chromosome movement, needs to be cleaved. Cohesin is removed by a protease, termed separase. However, separase activity is halted by another protein, known as securin (Peters, 2006). As mentioned earlier, the APC/C ubiquitinates securin and sends it to proteasomal degradation, thus starting the chain of degaradation events needed for chromosome segregation. Therefore, by keeping CDC20 inhibited, the SAC prevents premature exit from metaphase and later formation of lagging chromosomes (Bastians, 2015). 


\subsection{Microtubules}

\subsubsection{The dynamic instability of microtubules}

As already described, maintenance of genomic integrity is dependent on the properly assembled mitotic spindle. MTs form the mitotic spindle. They are divided into the following subsets: astral MTs ensure the spindle interactions with the cell cortex; kinetochore MTs tether the chromosomes to the mitotic spindle; interpolar MTs interdigitate between the spindle poles forming an array of antiparallel MTs named the "spindle midzone" (Fraschini, 2016).

Each MT consists of thirteen protofilaments arranged in a circle, and each protofilament is a polymer of $\alpha \beta$-tubulin dimers assembled in a head-to-tail fashion (Fraschini, 2016). As a result of the directionality in the organisation of each protofilament,

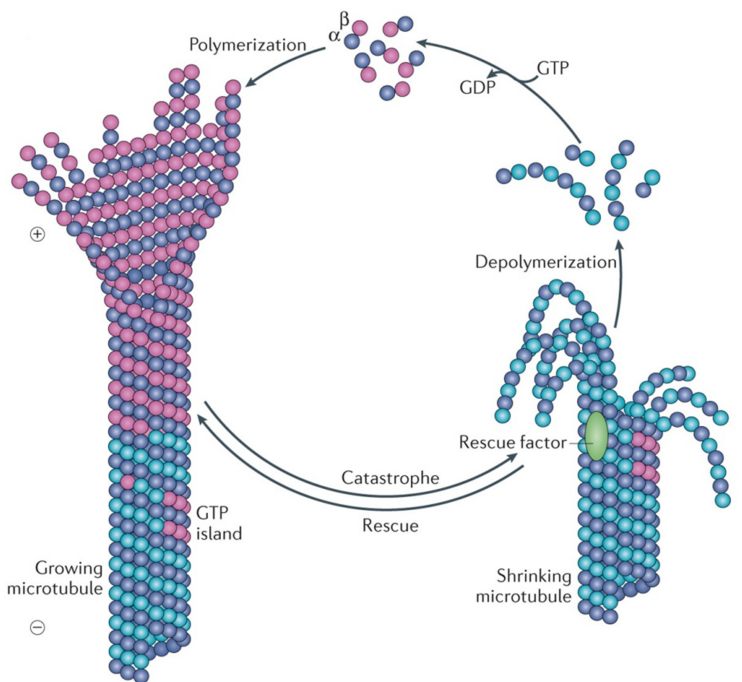

Figure 1.15: Microtubule dynamic instability. The MT growth (polymerisation) and shrinkage (depolymerisation) are driven by hydrolysis of the GTP bound to $\beta$-tubulin. New $G T P$-bound $\alpha \beta$-tubulin dimers are incorporated into growing MTs by hydrolysing the GTP which is already incorporated in the MT (at the tip), thus keeping the GTP intact. Once this GTP-cap is lost, due to the intrinsic instability of GDP-bound dimers, the MT growth will be rapidly switched to the shrinkage - a catastrophe. The rescue, which is a switch from shrinkage to growth, is possibly explained by the presence of "GTP islands" or "rescue factors" in the MT lattice. Adapted from (Akhmanova \&3 Steinmetz, 2015). the ends of MTs acquire distinct properties. One of the ends ( $\beta$-tubulin) is characterised by fast polymerisation, also described as growing properties, and therefore, is called the plus-end (or plus-tip). The opposite end ( $\alpha$-tubulin) is slow growing and is called the minus-end (or minus-tip).

The exquisite property of MTs is their ability of a fast switch between growth and shrinkage (depolymerisation), described as dynamic instability (Figure 1.15; Mitchison \& Kirschner, 1984). The switch from growth to shrinkage is denoted as a catastrophe, whereas the opposite way is called rescue (Gardner, Zanic, \& Howard, 2013). The GTP-cap model explains the current understanding of dynamic instability. The growing of the MTs is an energy- 
consuming process. The addition of each new $\alpha \beta$-dimer is driven by hydrolysis of a GTP molecule bound to $\beta$-tubulin at the plus-end. It is generally accepted that the stability of the newly formed MT tip is achieved by a so-called GTP cap, which contains the conformationally more stable GTP-tubulin, whereas the MT shaft contains the intrinsically unstable GDP-tubulin that is the MT grows as long as the GTP cap is present (Akhmanova \& Steinmetz, 2015).

The dynamic instability of MTs is essential for the proper functioning of the mitotic spindle. First, changes in the growth state itself create pulling and pushing forces, indispensable for the positioning and orienting the mitotic spindle (Grill \& Hyman, 2005; McNally, 2013; Tolić-Nørrelykke, 2008; H.-Y. Wu, Nazockdast, Shelley, \& Needleman, 2017). Moreover, different MT-interacting proteins bind the ends of MTs with different affinity, which, for instance, contributes to the directional movement of MT-bound motor proteins (Goodson \& Jonasson, 2018). The movement of MT-bound motor proteins generates forces that allow adjacent MTs to slide in relation to one another - a feature important for bipolar mitotic spindle formation and segregation of chromosomes (Fraschini, 2016). Last but not least, the dynamic instability underlies the "search and capture" model described above (Heald \& Khodjakov, 2015).

The switch between rescue and catastrophe events is a strictly regulated process. As depicted in Figure 1.16, a long list of proteins, including MAPs (microtubuleassociated proteins), +TIPs (plus-end-tracking proteins), -TIPs (minus-end-tracking proteins), are involved in the control of MT dynamics. Moreover, the activity of these proteins is tightly coordinated with cell cycle progression. An example of +TIPs is the family of MT polymerases XMAP215 (Xenopus microtubule-associated protein), which recruits and incorporates $\alpha \beta$-dimers in the plus-end of the MT (Brouhard et al., 2008). Therefore, misbalance in the activity of the members of this family can result in errors during mitosis. For instance, the MT polymerase ch-TOG (colonic and hepatic tumour overexpressed gene protein, also termed CKAP5) has been shown to be involved in the regulation of kinetochore-MT attachment stability, and changes in its activity can potentially lead to the formation of lagging chromosomes (Cheeseman, Harry, McAinsh, Prior, \& Royle, 2013; Miller, Asbury, \& Biggins, 2016). The end-binding proteins (EBs), which belong to + TIPs can moderately increase 


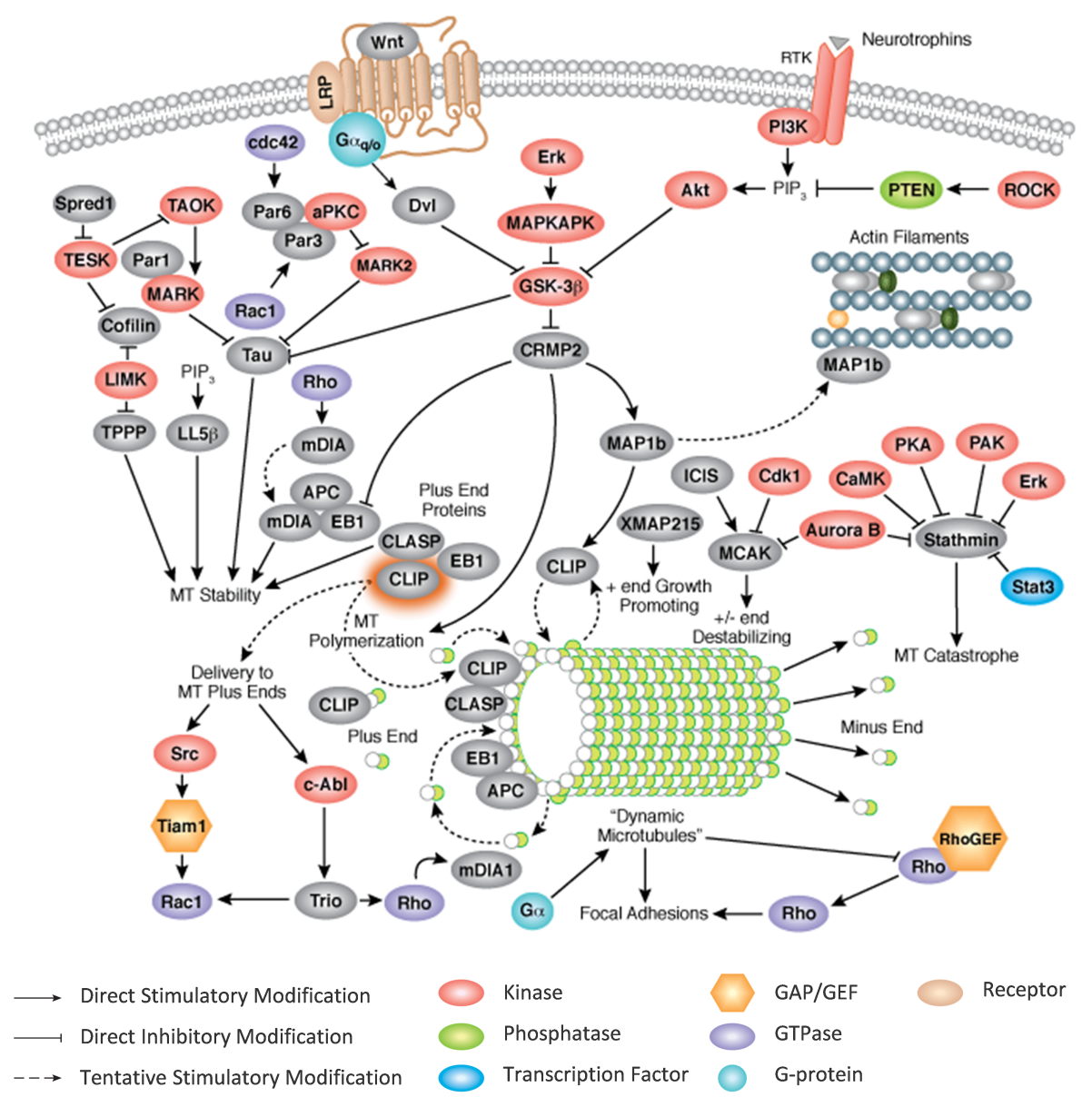

Figure 1.16: The complexity of regulation of microtubule dynamics. The diagram maps only some signalling pathways and the crosstalk between them, however, it can already be appreciated how strict and redundant the control of $M T$ dynamics is. In all cases, the entire process is narrowed down to the modulation of the polymerisation of MTs via recruiting rescue factors, changing the activity of XMAP215 MT polymerase or MCAK MT depolymerase. Also, involvement of cell cycle kinases, e.g. Aurora B and CDK1, is shown. Illustration reproduced courtesy of Cell Signaling Technology, Inc. (www.cellsignal.com).

MT polymerisation rates in vitro, possibly by influencing the stability of MT ends (Akhmanova \& Steinmetz, 2015; Lansbergen \& Akhmanova, 2006).

MT depolymerisation, or shrinkage, is regulated by the kinesin family of MT depolymerases, which are kinesin-13, kinesin-8 or kinesin-14 (Akhmanova \& Steinmetz, 2015; Walczak, Gayek, \& Ohi, 2013). Likewise, changes in the activity of these proteins are prone to generating errors during mitosis. Depletion of the kinesin- 8 KIF18A attenuates chromosome movements and delays the anaphase onset (Mayr et al., 2007; Stumpff, von Dassow, Wagenbach, Asbury, \& Wordeman, 2008; Stumpff, Wagenbach, Franck, Asbury, \& Wordeman, 2012). A member of the kinesin-13 family MCAK (mitotic centromere-associated kinesin) has been shown to be implicated in the alignment of chromosomes at metaphase (Illingworth, Pirmadjid, Serhal, Howe, 
\& Fitzharris, 2010) and sister chromatid segregation (Maney, Hunter, Wagenbach, \& Wordeman, 1998). Moreover, it has been shown that the major players of cell cycle Aurora B-PLK1 signalling regulates MCAK at kinetochores, thus ensuring the prompt correction of erroneous kinetochore-MT attachments and preventing formation of lagging chromosomes during anaphase (Shao et al., 2015).

\subsubsection{Microtubules in the presence of calcium}

Almost fifty years ago, studies carried out in vitro demonstrated that pure tubulin failed to polymerise in the presence of $\left[\mathrm{Ca}^{2+}\right]$ (Barnes, Engel, \& Dousa, 1975; Olmsted \& Borisy, 1975), and inhibition of polymerisation was readily reversible by addition of EGTA (ethylene glycol-bis(beta-aminoethyl)-N,N,N',N'-tetraacetic acid). Strikingly, incubation of brain extracts in the presence of $1 \mathrm{mM} \mathrm{CaCl}_{2}$ and MAPs (MAP1 and MAP2) resulted in the irreversible loss of tubulin polymerisation, despite the followedup addition of EGTA (Sandoval \& Weber, 1978). The authors showed that addition of MAP2 was sufficient to rescue tubulin polymerisation and that the observed effect was possibly due to the proteolytic action of a calcium-dependent protease specific for high-molecular-weight MAPs. Moreover, the presence of a calcium-dependent regulator (later identified as CaM; Sugden, Christie, \& Ashcroft, 1979) was shown to facilitate the effect of $\left[\mathrm{Ca}^{2+}\right]$ on MTs in depending on the concentration, yet it did not affect the polymerisation rate when the reaction was performed in the absence of calcium (Nishida, Kumagai, Ohtsuki, \& Sakai, 1979). Interestingly, when the polymerisation of tubulin was carried out in the presence of MAPs and calcium/CaM, the observed effect was similar as Nishida et al. had shown; yet when the MAPs were removed, the CaM enhanced the degree of pure tubulin polymerisation, probably by withdrawing $\mathrm{Ca}^{2+}$ from tubulin (Y. C. Lee \& Wolff, 1982). Later, it was shown that not only the presence of MAPs is important for the calcium-dependent effect, but also of Tau-proteins (Erneux, Passareiro, \& Nunez, 1984; Safinya et al., 2016).

It is clear that the effect of calcium on tubulin assembly in vivo will strongly depend on the MAPs and TIPs as well as other ions and proteins present (Humeau et al., 2018; O’Brien, Salmon, \& Erickson, 1997; Qin, Li, Yuan, \& Mao, 2012; Santella, 1998). Besides, calcium also causes morphological changes in MTs (V. Buljan, Ivanova, \& Cullen, 2009; V. A. Buljan et al., 2018; Safinya et al., 2016; Sataric, 
Sekulic, Sataric, \& Zdravkovic, 2015; Vater, Böhm, \& Unger, 1997). The picture gets even more complicated since the MTs change the cytosolic $\left[\mathrm{Ca}^{2+}\right]$ by modulating the SOCE (store operated calcium entry; Martin-Romero, Lopez-Guerrero, Pascual-Caro, \& Pozo-Guisado, 2017; Russa et al., 2008).

The importance of calcium in the cell cycle and apoptosis is well known and is summarised in Table 1.1 (Humeau et al., 2018)).

Table 1.1: Role of $\mathrm{Ca}^{2+}$ signal during the cell cycle (Humeau et al., 2018)

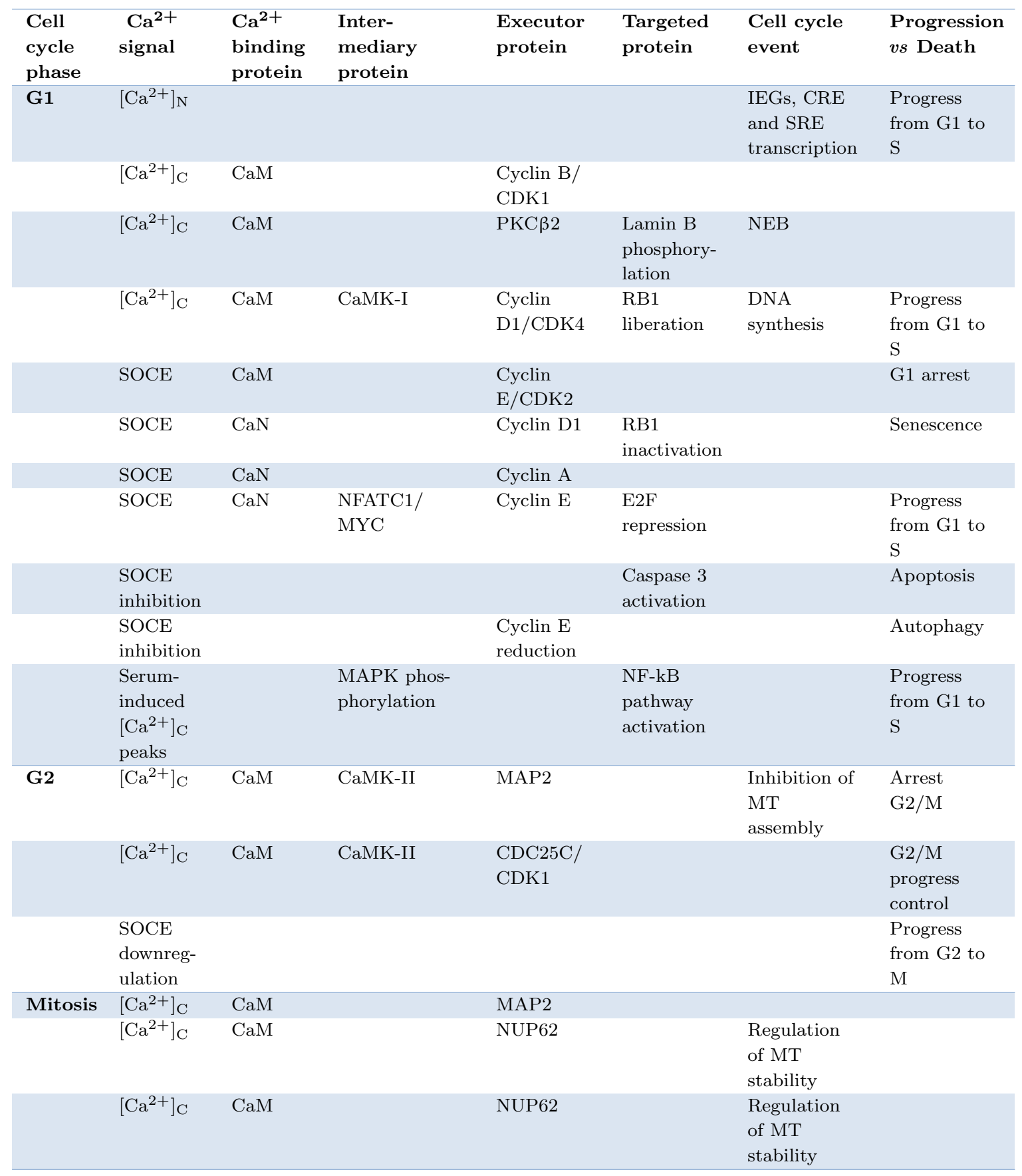


Table 1.1 (continued)

\begin{tabular}{|c|c|c|c|c|c|c|c|}
\hline $\begin{array}{l}\text { Cell } \\
\text { cycle } \\
\text { phase }\end{array}$ & $\begin{array}{r}\mathbf{C a}^{2+} \\
\text { signal }\end{array}$ & $\begin{array}{l}\mathrm{Ca}^{2+} \\
\text { binding } \\
\text { protein }\end{array}$ & $\begin{array}{l}\text { Inter- } \\
\text { mediary } \\
\text { protein }\end{array}$ & $\begin{array}{l}\text { Executor } \\
\text { protein }\end{array}$ & $\begin{array}{l}\text { Targeted } \\
\text { protein }\end{array}$ & $\begin{array}{l}\text { Cell cycle } \\
\text { event }\end{array}$ & $\begin{array}{l}\text { Progression } \\
\text { vs Death }\end{array}$ \\
\hline Mitosis & {$\left[\mathrm{Ca}^{2+}\right]_{\mathrm{N}}$} & & & RAD21 & & $\begin{array}{l}\text { Regulation } \\
\text { of MT } \\
\text { stability }\end{array}$ & \\
\hline & {$\left[\mathrm{Ca}^{2+}\right]_{\mathrm{N}}$} & & & CAPN & & $\begin{array}{l}\text { Sister } \\
\text { chromatid } \\
\text { separation }\end{array}$ & \\
\hline & {$\left[\mathrm{Ca}^{2+}\right]_{\mathrm{C}}$} & $\mathrm{CaM}$ & & MYLK & $\begin{array}{l}\text { MYL } \\
\text { phosphory- } \\
\text { lation }\end{array}$ & $\begin{array}{l}\text { Sister } \\
\text { chromatid } \\
\text { separation }\end{array}$ & \\
\hline & {$\left[\mathrm{Ca}^{2+}\right]_{\mathrm{C}}$} & $\mathrm{CaN}$ & & DNM2 & & $\begin{array}{l}\text { Cleavage } \\
\text { furrow } \\
\text { contraction }\end{array}$ & \\
\hline & {$\left[\mathrm{Ca}^{2+}\right]_{\mathrm{C}}$} & $\mathrm{CaM}$ & & CDK2 & CP110 & $\begin{array}{l}\text { Completion } \\
\text { of } \\
\text { cytokinesis }\end{array}$ & \\
\hline & {$\left[\mathrm{Ca}^{2+}\right]_{\mathrm{C}}$} & & & $\begin{array}{l}\text { Cyclin } \\
\text { B/CDK1 }\end{array}$ & & $\begin{array}{l}\text { Centrosome } \\
\text { duplication }\end{array}$ & $\begin{array}{l}\text { Exit from } \\
\text { mitosis }\end{array}$ \\
\hline
\end{tabular}


INTRODUCTION 


\section{The Aim of the Work}

Kv10.1 is explicitly expressed during the G2/M phase of the cell cycle. Moreover, the downregulation of Kv10.1 results in extended G2/M phase possibly through activation of the G2/M checkpoint. Nevertheless, the mechanism of Kv10.1-mediated cell cycle regulation, particularly at the G2/M transition, is still unknown.

In this context, this study aims to explain the molecular mechanism of the role of Kv10.1 in the regulation of G2 and M phases of the cell cycle. We hypothesise that Kv10.1 is involved in the regulation of at least one of the checkpoints acting at G2 and M phase. To confirm this hypothesis we utilised transient downregulation of Kv10.1 and specific blockers of the channel conductance. The objectives of the study were as follows:

1. Identification of the regulatory pathway or pathways which are affected by the loss of Kv10.1.

2. Finding the possible candidate or candidates which can interact either directly or through interposed factors with Kv10.1 and together modulate the progression through G2/M. 
The Aim of the Work 


\section{Materials \& Methods}

\subsection{Cell culture}

\subsubsection{Cell lines}

HeLa cells (ACC 57), acquired from the German Collection of Microorganisms and Cell Lines (DSMZ), were maintained in RPMI (Roswell Park Memorial Institute) 1640 Medium (GIBCO) supplemented with 10\% heat-inactivated FCS (fetal calf serum; Biochrom) at $37^{\circ} \mathrm{C}$ and $5 \% \mathrm{CO}_{2}$.

hTERT RPE1 (ATCC CRL4000) were obtained from ATCC and maintained in DMEM/F-12 (Dulbecco's Modified Eagle Medium: Nutrient Mixture F-12; GIBCO) supplemented with $10 \%$ heat-inactivated FCS and $0.01 \mathrm{mg} / \mathrm{ml}$ hygromycin $\mathrm{B}$ at $37^{\circ} \mathrm{C}$ and $5 \% \mathrm{CO}_{2}$.

\subsubsection{Passaging of cells}

HeLa and hTERT RPE1 cells were routinely passaged once they reached $80-$ 90\% confluency. The cells were washed with DPBS (Dulbecco's Phosphate Buffered Saline; GIBCO) and trypsinised by incubating with Trypsin-EDTA (0.05\%:0.02\%, w:v; Biochrom) for 5 min at $37^{\circ} \mathrm{C}$. The trypsin activity was neutralised by addition of the corresponding full growth medium at 3:1 (v:v) ratio of added Trypsin-EDTA. The cells were then harvested by centrifuging at $300 \mathrm{x} g$ for $2 \mathrm{~min}$, resuspended with fresh medium and plated at the required density.

\subsubsection{Cell count}

The cell count was determined using Neubauer's chamber. Briefly, an aliquot of cell suspension was mixed with Trypan Blue (Sigma) at 1:1 (v:v) ratio, resuspended using a pipette, and $10 \mu \mathrm{l}$ of the suspension was introduced into the chamber. Only Trypan Blue-negative cells were counted inside of the four large squares. If the cells 
were on the borderlines of the squares, only the ones crossing the upper and left border lines were counted. The cell concentration was then derived from the counted cell number using the following formula:

$$
\text { Concentration }(\mathrm{cell} / \mathrm{ml})=\frac{\text { number of cells } * \text { dilution factor } * 10000}{\text { number of squares }}
$$

The final dilution was adjusted in order to obtain $1 * 10^{6}$ cells $/ \mathrm{ml}$.

\subsubsection{Plasmid DNA purification}

Visualisation of growing MTs and overexpression of Kv10.1 was achieved by transfecting the cells with pEB3 (end-binding protein 3)-tdTomato (a gift from Erik Dent; plasmid \#50708, Addgene; Merriam et al., 2013) and hEAG1-ptracer (Pardo et al., 1999), respectively.

Before the purification, pDNA was first inoculated into bacterial cells and grown to yield higher amounts of the pDNA. In brief, $1 \mu \mathrm{l}$ of a pDNA was added to 50 $\mu \mathrm{l}$ of DH5-alpha competent cell suspension, gently resuspended with a pipette, and incubated for $5 \mathrm{~min}$ on ice. Then heat-shock was applied to the cells by first incubating them for $1 \mathrm{~min}$ in a $42^{\circ} \mathrm{C}$ water bath, then for $10 \mathrm{~min}$ on ice. After that, the cell suspension was extended onto an agar coated dish with the respective antibiotic and incubated at $37^{\circ} \mathrm{C}$, overnight. The choice of the antibiotic was defined by an antibiotic resistance gene present on the pDNA being introduced into the bacterial cells. Thus, only bacteria carrying the plasmid will be able to grow under such conditions (Voß, 2007). The cells inoculated with pEB3-tdTomato were cultivated in the presence of kanamycin $(25 \mu \mathrm{g} / \mathrm{ml})$, and hEAG1-ptracer - ampicillin. On the following day, a single colony of bacterial cells was collected and further incubated in $100 \mathrm{ml}$ of liquid LB (Luria Broth; $1 \% \mathrm{NaCl}, 1 \%$ Tryptone, $0.5 \%$ yeast extract) medium with the respective antibiotic at $37^{\circ} \mathrm{C}$ and $300 \mathrm{rpm}$ for $12-16 \mathrm{~h}$.

The pDNA was purified using NucleoBond Xtra Midi EF endotoxin-free plasmid DNA purification kit (MACHEREY-NAGEL) following the manufacturer's instructions. In brief, the bacterial cells were centrifuged for $15 \mathrm{~min}$ at $6000 \mathrm{x} g$ and $4^{\circ} \mathrm{C}$. The harvested cells were resuspended in $8 \mathrm{ml}$ of Resuspension Buffer RES-EF containing RNase A and lysed with $8 \mathrm{ml}$ of Lysis Buffer LYS-EF by gently inverting the tube 5 times and incubating at RT (room temperature) for $5 \mathrm{~min}$. Meanwhile, 
the NucleoBond Xtra Column was equilibrated with $15 \mathrm{ml}$ of Equilibration Buffer EQU-EF. The resulting crude lysate was neutralised with $8 \mathrm{ml}$ of Neutralisation Buffer NEU-EF, mixed gently by inverting the tube several times, and loaded onto the NucleoBond Xtra Column. Once the column emptied by gravity flow, the NucleoBond Xtra Column and NucleoBond Extra column Filter were first rinsed with $5 \mathrm{ml}$ of Filter Wash Buffer FIL-EF. After that, the filter was discarded, and the column was washed first with $35 \mathrm{ml}$ of Wash Buffer ENDO-EF, and then with $15 \mathrm{ml}$ of Wash Buffer WASH-EF. After each step the column was let empty as before. The plasmid DNA was eluted with $5 \mathrm{ml}$ of Elution Buffer ELU-EF into $15 \mathrm{ml}$ centrifuge tubes (Beckman Coulter). The eluted plasmid DNA was precipitated with isopropanol at RT, vortexed thoroughly and pelleted by centrifuging for $30 \mathrm{~min}$ at $15000 \mathrm{xg}$ and $4^{\circ} \mathrm{C}$. The supernatant was decanted carefully, and the DNA pellet was washed with $70 \%$ ethanol. Afterwards, the plasmid DNA pellet was allowed to dry at RT under a laminar hood, and reconstituted by dissolving it in an appropriate volume of endotoxin-free $\mathrm{H}_{2} \mathrm{O}-\mathrm{EF}$. Plasmid DNA yield and purity was determined by measuring the absorbance in the UV-range, and the plasmid integrity was confirmed by agarose gel electrophoresis. The purified pDNA was aliquoted and stored at $-20^{\circ} \mathrm{C}$.

\subsection{5 siRNA and pDNA transfection}

Complexation, cell entry and intracellular delivery of nucleic acids define the underlying principle of transfection. The complexation step is based on the interaction of positively and negatively charged particles. Due to the polyphosphate backbone, nucleic acids are negatively charged and, therefore, can interact with positively charged transfection reagents. As a result positively charged transfection complexes are formed which both protect nucleic acids from nuclease degradation and ease cell entry. Positively charged transfection complexes can interact with the negatively charged heparan sulphate proteoglycans present on the outer surface of the cytoplasmic membrane of adherent cells. The exact mechanism of entry of the transfection complexes into the cell is unclear, but it is believed that the interaction with cytoplasmic membrane triggers their inclusion via endocytosis. An ability of transfection reagents to induce rupture or fusion of the vesicular membrane and so release the nucleic acids into the cytoplasm determines their effectiveness. Most 
nucleic acids can already function in the cytoplasm. In the case of generating stable mutations, the nucleic acids should be delivered into the nucleus and integrated into the cell genome (Dalby et al., 2004; Kim \& Eberwine, 2010).

Transient downregulation of Kv10.1 in HeLa and hTERT RPE1 cells was achieved by siRNA transfection. The following siRNA sequence directed against KCNH1 was used: 5'-TACAGCCATCTTGGTCCCTTA-3' (HP Custom siRNA, Qiagen). A commercial negative control (scrambled, Scr) siRNA (cat \# AM4635, Ambion) was used as a control for siRNA interference. The cells were plated at least $24 \mathrm{~h}$ before the transfection, and were $50-70 \%$ confluent at the moment of transfection. Optimal downregulation or overexpression was achieved by using different transfection reagents depending on the cell type and the target protein.

\section{siRNA interference using Lipofectamine RNAiMAX}

siRNA transfection in hTERT RPE1 cells was carried out using Lipofectamine RNAiMAX transfection reagent (Invitrogen). Briefly, two working solutions were prepared. Solution one contained the respective siRNA at a final concentration of $30 \mathrm{nM}$ in $125 \mu \mathrm{l}$ OptiMEM-I+GlutaMAX-I (GIBCO) per well of a 6-well culture plate. For the second solution, $3 \mu$ l of Lipofectamine was mixed with $122 \mu \mathrm{l}$ of OptiMEM-I+GlutaMAX-I per well. Both solutions were briefly vortexed and spun down. Diluted siRNA was then added to the lipofectamine-containing solution, pipette-mixed and incubated for $15 \mathrm{~min}$ at RT. The transfection mixture was added to the cells growing in a 6 -well plate in $2 \mathrm{ml}$ normal growth medium, resulting in the final volume of $2.25 \mathrm{ml}$ per well. The cells were incubated in the presence of the transfection mixture for $4 \mathrm{~h}$ at $37^{\circ} \mathrm{C}$ and $5 \% \mathrm{CO}_{2}$. The medium was subsequently changed, and after $48 \mathrm{~h}$, the transfected cells were used for the experiments. The efficiency of the siRNA interference was tested by immunoprecipitation and WB (western blot) at least for one experimental repeat of experiments of different designs.

\section{siRNA interference using INTERFERin}

Transient downregulation of Kv10.1 in HeLa cells was achieved using INTERFERin transfection reagent (Polyplus-transfection SA) following the manufacturer's instructions. For this, the amount of siRNA corresponding to $30 \mathrm{nM}$ final concen- 
tration was added to $190 \mu \mathrm{l}$ of OptiMEM per well of a 6 -well plate; $10 \mu \mathrm{l} /$ well of INTERFERin was then added to the diluted siRNA solution and immediately vortexed for $10 \mathrm{sec}$. The siRNA-INTERFERin mixture was spun down and incubated for $10 \mathrm{~min}$ at RT. The final volume of the transfection mixture, $200 \mu \mathrm{l} /$ well, which was added dropwise to the cells growing in $2 \mathrm{ml}$ standard growth medium. The cells were incubated with the transfection mixture for at least $2 \mathrm{~h}$ at $37^{\circ} \mathrm{C}$ and $5 \% \mathrm{CO}_{2}$. Subsequently, the medium containing the transfection mixture was removed and the cells were further cultivated in fresh medium.

\section{pDNA transfection using jetPRIME}

In order to overexpress a protein of interest in HeLa and hTERT RPE1 cells, pDNA (plasmid DNA) was introduced into the cells using jetPRIME transfection reagent (Polyplus-transfection SA). The procedure in brief was as follows: $1 \mu \mathrm{g}$ of DNA was mixed with jetPRIME buffer and $2 \mu \mathrm{l}$ of jetPRIME. The volume of jetPRIME buffer was adjusted to $400 \mu \mathrm{l}$ final volume. Immediately after that, the mixture was vortexed for $10 \mathrm{~s}$, spun down and incubated at RT for $10 \mathrm{~min}$. Afterwards, $100 \mu \mathrm{l}$ of the transfection mixture was added dropwise to each well of a 4-well $\mu$-slide (ibiTreat, \#1.5, Ibidi), and incubated for $4 \mathrm{~h}$ at $37^{\circ} \mathrm{C}$ and $5 \% \mathrm{CO}_{2}$. Then, the cells were supplemented with fresh growth medium and incubated at least $24 \mathrm{~h}$ at $37^{\circ} \mathrm{C}$ and $5 \% \mathrm{CO}_{2}$.

\section{pDNA and siRNA co-transfection with jetPRIME}

Transient overexpression of one protein and simultaneous downregulation of another protein was achieved by co-transfecting pDNA and siRNA, using jetPRIME (Polyplus-transfection SA). In brief, HeLa and hTERT RPE1 cells were seeded in a 6 -well plate $24 \mathrm{~h}$ before the co-transfection. The numbers below are given per well of a 6 -well plate. On the day of transfection, the pDNA and siRNA were diluted with jetPRIME buffer to obtain $1 \mu \mathrm{g} /$ well and $30 \mathrm{nM}$ final concentrations, respectively. Then, $10 \mu \mathrm{l}$ of jetPRIME transfection reagent was added to the diluted samples, vortexed for $10 \mathrm{~s}$, spun down and incubated for $10 \mathrm{~min}$ at RT. Meanwhile, the medium in each well was replaced with $2 \mathrm{ml}$ of the appropriate growth medium. The transfection mixture was added to the each well dropwise. The cells were 
incubated with the transfection mixture for $4 \mathrm{~h}$ at $37^{\circ} \mathrm{C}$ and $5 \% \mathrm{CO}_{2}$, and then the medium was substituted with a fresh one. The cells were used in experiments $48 \mathrm{~h}$ post-transfection.

\subsubsection{Cell cycle synchronisation}

\section{Synchronisation in G0 phase by serum deprivation}

One of the commonly used methods for synchronising cells in the G0 quiescent phase of the cell cycle is the serum deprivation or starvation approach. It is based on the requirement of mitogens for cells to progress through the restriction point (Pardee, 1974). Once cells pass the restriction point, they enter the $\mathrm{S}$ phase and complete the cell cycle without further stimulation by mitogens (Reed, 1997; Zetterberg \& Larsson, 1985). Due to the absence of mitotic signals, the cells, which are in G1 phase at the moment of starting serum deprivation, exit from the cell cycle into a non-dividing state, termed G0 and characterised by low metabolic state. Cells that already have passed the restriction point will proceed further through the cell cycle until they reach the G1 phase again, where the absence of mitogens in the environment will force them to enter the G0 phase (Iyer et al., 1999).

This method was used to synchronise hTERT RPE1 cells at the G0/G1 border. The cells were cultured until $65-70 \%$ confluency in T-75 flasks in the corresponding growth medium. The synchronisation of the cells was then started: the serum containing growth medium was removed and the cells were washed twice with growth medium with no FCS. Thus, the residual mitogens are eliminated, and the cells were then exposed to serum-free medium: DMEM/F-12 supplemented only with $0.01 \mathrm{mg} / \mathrm{ml}$ hygromycin. The cells were starved for $60 \mathrm{~h}$ at $37^{\circ} \mathrm{C}$ and $5 \% \mathrm{CO}_{2}$. The hTERT RPE1 cells synchronised in such manner were used for intracellular calcium imaging.

\section{Synchronisation at G1/S border by double thymidine block}

To study the different stages of the cell cycle, HeLa cells were synchronised by double thymidine block. At the basis of thymidine block lies the negative regulation of nucleotide synthesis caused by disruption of the nucleotide pool sizes. Treatment of cells with an excess of thymidine $(2 \mathrm{mM})$ leads to allosteric inhibition of class I 
A

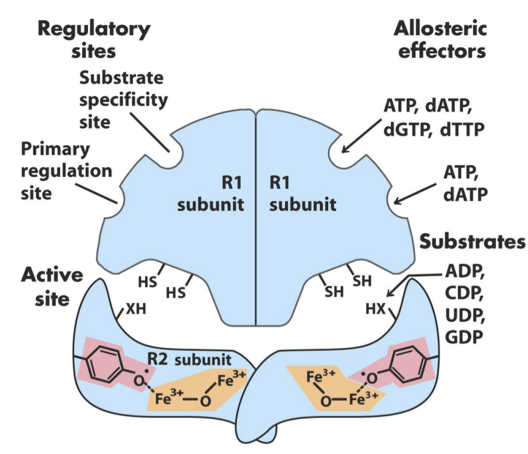

B

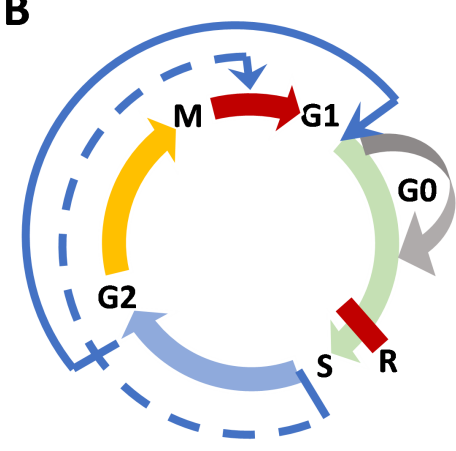

C

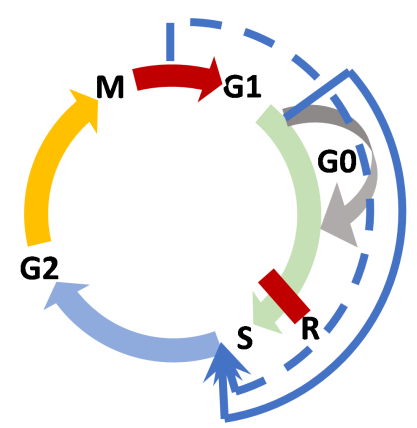

Figure 2.1: The principle of double thymidine block. A, Regulation of class I ribonucleotide reductase (RNR1). Class I RNRs activity is regulated by binding ATP (activation) or dATP (inactivation). Active enzyme reduces the substrates according to the corresponding effector molecules (dTTP, dGTP, dATP, ATP) bound to the allosteric sites. In this manner, the substrate specificity regulation ensures equal production of dNTPs necessary for DNA replication. Disruption in this balanced state leads to the inhibition of the enzyme and cell cycle arrest at G1/S border (Jordan 83 Reichard, 1998). B and C, The mechanism of the double thymidine block is illustrated. $B$, The cells which at the beginning of the synchronisation were already in $S$ phase, or G1 or late G2 at the end of the first block with thymidine will become arrested at different stages of $S$ phase. Wash-out of the thymidine and exposure to a fresh growth medium allows the cells to exit the S-phase and reach late G2 or early G1 phase (J. V. Harper, 2005). C, Subsequently, the second block with thymidine results in the arrest of the cells at the $G 1 / S$ border (the dashed arrow indicates the propagation of the cells arrested in G1/S and early $S$ phase and the solid arrow indicates the propagation of the cells in late $S$ phase during the release from the first block; $R$ is the restriction point; J. V. Harper, 2005).

RNR (ribonucleotide reductase), resulting in inhibition of DNA synthesis and arrest of the cells at the G1/S border (Figure 2.1; Cory \& Sato, 1983). The necessity of two consecutive exposures to the excess of thymidine is depicted in Figure 2.1. In brief, during the first block with thymidine excess, which in case of HeLa cells lasts $16 \mathrm{~h}$, some of the cells will be arrested at the G1/S border. However, those cells that were already in $\mathrm{S}$ phase when thymidine was applied, will be arrested along $\mathrm{S}$ phase. Following release from the thymidine block for $8 \mathrm{~h}$, the cells that were arrested at the G1/S border will progress until G2/M, and the cells arrested at different stages of S phase will cycle until G1 phase. Thereby, during the second exposure to the excess of thymidine for another $16 \mathrm{~h}$, a vast majority of the cells will accumulate at the G1/S border (J. V. Harper, 2005). Of note, the length of each step described above is strongly dependent on the cell cycle duration of cells in question, and time intervals given here correspond to the experiments using HeLa cells. HeLa cells blocked at G1/S in such manner were then released into fresh growth medium with $10 \%$ FCS, and cell samples were collected every $2 \mathrm{~h}$ between $0-6 \mathrm{~h}$ and $10-14 \mathrm{~h}$, and every 1 h between 6 - 10 h (G2/M phase) after the release and further used for biochemistry. 


\section{Synchronisation in prophase by dimethylenastron}

The MT dynamics were investigated in HeLa and hTERT RPE1 cells synchronised in prophase. It has been shown that the small-molecule DME (dimethylenastron) can be a useful tool for studying MT dynamics in fixed or live cells (Ertych et al., 2014). DME is an analogue of monastrol, which inhibits a motor kinesin Eg5 (also known as KIF11; Müller et al., 2006). Eg5 is essential for bipolar spindle formation by generating forces needed for establishing and maintaining the bipolar spindle and contributing to its elongation (Mann \& Wadsworth, 2019). Inhibition of Eg5 with DME leads to the arrest of the cells in prophase and formation of monopolar spindles (Mayer et al., 1999). Interestingly, cells with altered MT dynamics form asymmetric monopolar spindles. It should be noted that the treatment with DME itself has no effect on the assembly rates of MT plus-tips (Ertych et al., 2014). Quantification of these structures can already hint at the potential alterations in MT dynamics, which should be further validated by a direct measurement of MT dynamics.

Asynchronously growing HeLa and hTERT RPE1 cells were incubated with 2.5 $\mu \mathrm{M}$ DME for $4 \mathrm{~h}$ at $37^{\circ} \mathrm{C}$ and $5 \% \mathrm{CO}_{2}$. Afterwards, the cells were either used for live cell imaging or fixed for further immunofluorescence staining.

Synchronisation in metaphase and anaphase by thymidine-nocodazole treatment

Enrichment of the cell populations in metaphase and anaphase was achieved by sequential treatment with thymidine and nocodazole, followed by either treatment with the proteasome inhibitor MG132 or release into the growth medium, respectively. As already described above, treatment with an excess of thymidine only once results in the arrest of the cells at the G1/S border and along $\mathrm{S}$ phase.

The second part of the protocol is based on the MT depolymerising property of nocodazole (methyl [5-(2-thienylcarbonyl)-1H-benzimidazol-2-yl] carbamate), also known as oncodazole (Friedman \& Platzer, 1978; Hoebeke, Van Nijen, \& De Brabander, 1976; Ireland, Gull, Gutteridge, \& Pogson, 1979). MT formation is an important structural feature of cells, especially during mitosis. They participate in the exact organisation and function of the mitotic spindle, and are critical for assuring the integrity of the segregated DNA (Abal, Andreu, \& Barasoain, 2003). It has been 
shown that nocodazole interacts and chemically modifies tubulin by preventing its polymerisation, and the presence of MAPs does not amplify the inhibitory effect of the drug (J. C. Lee, Field, \& Lee, 1980). Several other drugs, such as vincristine and colcemid, similar to nocodazole interfere with MTs and cause arrest in G2/M (Florian \& Mitchison, 2016). However, interaction of nocodazole with MTs is rapid and readily reversible, which makes it potentially useful for synchronisation of the cells (Zieve, Turnbull, Mullins, \& McIntosh, 1980). In addition, nocodazole as an agent for synchronising cells at G2/M has been shown to be most efficient and less harmful for the cells when applied to a presynchronised population, e.g. metabolically blocked G1/S cells (Nüsse \& Egner, 1984).

In brief, HeLa cells were first incubated with $2 \mathrm{mM}$ thymidine for $16 \mathrm{~h}$ at $37^{\circ} \mathrm{C}$ and $5 \% \mathrm{CO}_{2}$. Then, thymidine containing medium was removed, and the cells were rinsed twice with DPBS and released into a fresh growth medium for $4 \mathrm{~h}$, allowing them to enter the G2 phase. Afterwards, HeLa cells were treated with medium containing $2 \mathrm{ng} / \mathrm{ml}$ nocodazole for an additional $4 \mathrm{~h}$, followed by a washing step as described above. HeLa cells synchronised at prophase in such manner were further used either for enriching them in metaphase or anaphase. In case, of anaphase, the cells were further incubated 40 min with a standard growth medium.

Regulation of transition through different phases of cell cycle is achieved via ubiquitination-mediated degradation of cyclins (Gilberto \& Peter, 2017; Zheng, Wang, \& Wei, 2016). Of particular interest is the regulation of the transition from metaphase-to-anaphase via APC/C mediated degradation of cyclin B1 (Poon, 2016; Reed, 2006). Proteasome inhibitors can be a valuable tool for studying this step of cell cycle (D. H. Lee \& Goldberg, 1998). MG132 (carbobenzoxy-Leu-Leu-leucinal), a peptide aldehyde, is one of such inhibitors, and it effectively blocks the proteolytic activity of the 26S proteasome complex (D. H. Lee \& Goldberg, 1996; Rock et al., 1994). Therefore, in our experiments accumulation of cells at metaphase was achieved by additionally incubating HeLa cells with $15 \mu \mathrm{M}$ MG132 for $2 \mathrm{~h}$ at $37^{\circ} \mathrm{C}$ and $5 \%$ $\mathrm{CO}_{2}$ after the thymidine-nocodazole treatment.

Cells synchronised at metaphase and anaphase were further fixed and immunostained for assessment of chromosomal alignment at metaphase and presence of lagging chromosomes at anaphase as described in the following paragraphs. 


\subsection{Biochemistry}

\subsubsection{Protein extraction}

Cells were harvested by trypsinisation and centrifuged for 2 min at $300 \mathrm{xg}$ for protein isolation. Afterwards, the supernatant was removed and the cell pellets were lysed in non-denaturing lysis buffer (1\% Triton X-100, 50 mM Tris-HCl, 300 mM $\mathrm{NaCl}, 5 \mathrm{mM}$ EDTA) containing protease and phosphatase inhibitor cocktail (Roche) for 30 min at RT in 1:3 ratio (v:v). For nuclear protein extraction, a modified RIPA buffer was used: 1\% (v:v) NP-40 (Nonidet P-40, octylphenoxypolyethoxyethanol), $0.1 \%$ (m:v) Na deoxycholate, $300 \mathrm{mM} \mathrm{NaCl}, 50 \mathrm{mM}$ Tris-HCl. After the lysis, the samples were centrifuged at $18000 \mathrm{x} g$ for $15 \mathrm{~min}$ at $4^{\circ} \mathrm{C}$. The supernatant was then recovered and stored at $-80^{\circ} \mathrm{C}$ until further use.

\subsubsection{Protein concentration determination}

Protein concentration was determined with the BCA (bicinchoninic acid) assay. This assay, also termed Smith assay after its inventor Paul K. Smith (P. K. Smith et al., 1985), determines the total concentration of proteins in a solution $(0.5 \mu \mathrm{g} / \mathrm{ml}$ to $1.5 \mathrm{mg} / \mathrm{ml}$ ). The BCA assay mainly consists of two reactions: first, the reduction of bivalent copper ions to monovalent state by peptide bonds in a temperaturedependent manner (Wikipedia, n.d.). Therefore the amount of reduced $\mathrm{Cu}^{2+}$ is directly transferable to the amount of proteins present in the solution. The second reaction is the complexion of two bicinchoninic acid molecules with a monovalent copper ion. This complex depends on the availability of tyrosine, cysteine/cystine, and tryptophan in the measurable proteins, and at high temperatures $\left(37\right.$ to $\left.60^{\circ} \mathrm{C}\right)$ is purple-coloured with the absorbance peak at $562 \mathrm{~nm}$. Hence, the protein concentration can be determined by measuring the absorbance and extrapolating it from the absorbance values of protein solutions with known concentration (Walker, 1994; Wikipedia, n.d.).

BCA Protein Assay was performed following the manufacturer's instructions, calibrated with bovine serum albumin (BSA; Thermo Fisher Scientific). In brief, an aliquot of the protein sample was diluted 5 times with respective lysis buffer to obtain $40 \mu \mathrm{l}$ final volume. Then, $10 \mu \mathrm{l}$ of the diluted protein sample was pipetted into a 
well of a 96-well plate, in triplicates. The reaction buffer was prepared by mixing 50 parts of solution A (contains sodium carbonate, sodium bicarbonate, bicinchoninic acid and sodium tartrate in $0.1 \mathrm{M}$ sodium hydroxide) with 1 part of solution $\mathrm{B}$ (contains $4 \%$ cupric sulfate), and $200 \mu \mathrm{l}$ of that was added to each well. The reaction mixture was then incubated for $30 \mathrm{~min}$ at $37^{\circ} \mathrm{C}$. Afterwards, the samples were cooled down to RT, and the absorbance at $562 \mathrm{~nm}$ was measured on Wallac 1420 VICTOR2 microplate reader (PerkinElmer, Inc., USA).

\subsubsection{SDS-PAGE}

Proteins were separated according to their molecular weight using SDS-PAGE (sodium dodecyl sulphate polyacrylamide gel electrophoresis). In order to disrupt higher order protein structures and prevent disulphide bridge formation, NuPAGE Reducing agent and NuPAGE LDS Sample buffer (Invitrogen) were added to the samples $(50 \mu \mathrm{g}$ total proteins in $30 \mu \mathrm{l}$ final volume) in 1:10 (v:v) and 1:4 (v:v) ratio, respectively. The samples were then denatured at $70^{\circ} \mathrm{C}$ for $10 \mathrm{~min}$.

The denatured samples were resolved on $4-12 \%$ NuPAGE Novex Bis-Tris Mini Gels (1.0 mm, 12 well; Invitrogen) at $200 \mathrm{~V}$ in MOPS electrophoresis buffer. For high molecular weight proteins, 3 - 8\% NuPAGE Novex Tris-Acetate Gels $(1.5 \mathrm{~mm}$, 10 well; Invitrogen) and Tris-Acetate electrophoresis buffer (Invitrogen) were used. The molecular weight of the protein of interest was identified with the following prestained protein standards: ColorPlus (New England BioLabs) or All Blue (Bio-Rad) - for low- and mid-range molecular weight proteins, and Hi-MARK (Thermo Fischer Scientific) - for high molecular weight proteins.

\subsubsection{Immunoblotting}

Once protein samples were separated by SDS-PAGE, the proteins were transferred onto an NC (nitrocellulose) membrane using a tank (wet) electro-transfer procedure.

Low- and mid-range molecular weight protein. The protein samples were blotted onto a pre-cut NC membrane $(0.2 \mu \mathrm{m}$; Invitrogen $)$ at $50 \mathrm{~V}$ for $2 \mathrm{~h}$ at RT. The following transfer buffer was used: $10 \mathrm{mM} \mathrm{NaHCO} 3,3 \mathrm{mM} \mathrm{Na} \mathrm{CO}_{3}, 0.01 \%$ SDS and $20 \%$ methanol.

High molecular weight protein. The protein samples were blotted onto a pre-cut 
NC membrane $\left(0.45 \mu \mathrm{m}\right.$; GE Healthcare) at $35 \mathrm{~V}$ and $4^{\circ} \mathrm{C}$, overnight. The composition of the transfer buffer was the same, except that it contained $15 \%$ methanol.

After the accomplishment of the transfer, the NC membranes were rinsed with $\mathrm{ddH}_{2} \mathrm{O}$ (deionised water). The membranes were then treated with Blot Signal Enhancer (Thermo Scientific) according to the manufacturer's instructions. In brief, the membranes were first incubated with Solution 1 for 2 min at RT and protected from light; then rinsed with $\mathrm{ddH}_{2} \mathrm{O} 5$ times, and incubated with Solution 2 for 10 min at RT. After that, the membranes were again rinsed with $\mathrm{ddH}_{2} \mathrm{O}$ five times and twice with TBS. The membranes were stored in TBS at $4^{\circ} \mathrm{C}$ or further processed.

The membranes were incubated with $0.1 \%$ casein (Roche) in TBST (20 mM Tris, $150 \mathrm{mM} \mathrm{NaCl}$ and $0.1 \%$ Tween 20) for $1 \mathrm{~h}$ at RT and gentle agitation, which will later prevent non-specific binding of the antibody probes to the membrane surface. Taking into account that casein can be phosphorylated, and thus it can compete with the phosphorylated antigen for antibody binding, 5\% BSA in TBST was used for detection of phosphoproteins. Proteins of interest were identified by incubating the NC membranes with a primary antibody (Table 2.1) diluted in the corresponding blocking buffer overnight at $4^{\circ} \mathrm{C}$ with gentle agitation. On the following day, the primary antibody solution was recovered, and the excess was removed from the membranes by washing 5 times with $\mathrm{ddH}_{2} \mathrm{O}$ and twice with TBST. Afterwards, the membranes were incubated with HRP (horseradish peroxidase)-conjugated secondary antibody diluted in blocking buffer for 45 min at RT with gentle agitation (Table 2.1). Then the membranes were washed as above, and incubated with $5 \mathrm{ml}$ of a chemiluminescent HRP substrate (Millipore) for 5 min at RT with gentle agitation and protected from light. The substrate triggers secondary antibody-conjugated HRP-catalysed chemiluminescence reaction. The emitted light was then detected with a ChemiDoc XRS system (Bio-Rad) controlled by Quantity One 1-D Analysis software v4.6.9 (Bio-Rad). Images were acquired starting from $1 \mathrm{~s}$ of exposure time until 10 min of total exposure and taking 10 images per membrane. If a saturated signal was detected, then the image acquisition was terminated.

After detection of the protein of interest, the antibodies bound to the membrane were removed with Restore Plus Western Blot stripping buffer (Life technologies). Restore Plus Western Blot stripping buffer was added to each membrane (approxi- 
mately $20 \mathrm{ml}$ for an $8 \times 10 \mathrm{~cm}$ blot) and incubated for $30 \mathrm{~min}$ at RT with gentle agitation. The complete removal of enzyme conjugates was tested by additionally incubating with an HRP chemiluminescence substrate. If no signal was detected during a 5 min exposure, the membranes were ready for re-probing with new primary and secondary antibodies, preceded by a blocking step.

Table 2.1: List of primary and secondary antibodies used for immunoblotting

\begin{tabular}{|c|c|c|c|c|}
\hline Antibody & Source & Dilution & $\begin{array}{l}\text { Cat. } \\
\text { Number }\end{array}$ & Manufacturer \\
\hline p-ATR (Ser428) & Rabbit/polyclonal & $1: 1000$ & 2853 & Cell Signaling \\
\hline p-BRCA1（Ser1524） & Rabbit/polyclonal & $1: 1000$ & 9009 & Cell Signaling \\
\hline p-CHK1 (Ser345) (133D3) & Rabbit/monoclonal & $1: 1000$ & 2348 & Cell Signaling \\
\hline CHK1 (FL-476) & Rabbit/polyclonal & $1: 1000$ & sc-7898 & $\begin{array}{l}\text { Santa Cruz } \\
\text { Biotechnology }\end{array}$ \\
\hline p-CHK2 (Thr68)(C13C1) & Rabbit/monoclonal & $1: 1000$ & 2197 & Cell Signaling \\
\hline CHK2 (DCS-270) & Mouse/monoclonal & $1: 1000$ & sc-56296 & $\begin{array}{l}\text { Santa Cruz } \\
\text { Biotechnology }\end{array}$ \\
\hline p21 Waf1/Cip1 & Mouse/monoclonal & $1: 1000$ & $05-345$ & Merck Millipore \\
\hline p-histone H2AX (Ser139) & Rabbit/monoclonal & $1: 1000$ & sc-101696 & Cell Signaling \\
\hline CDC27 (AF3.1) & Mouse/monoclonal & $1: 1000$ & sc-9972 & $\begin{array}{l}\text { Santa Cruz } \\
\text { Biotechnology }\end{array}$ \\
\hline p-CDC25C (S216) & Rabbit/monoclonal & 1:1000 & ab32051 & Abcam \\
\hline CDC25C & Rabbit/polyclonal & $1: 1000$ & sc-327 & $\begin{array}{l}\text { Santa Cruz } \\
\text { Biotechnology }\end{array}$ \\
\hline CDC20 & Rabbit/polyclonal & $1: 1000$ & ab26483 & Abcam \\
\hline Aurora B/AIM1 & Rabbit/polyclonal & $1: 1000$ & 3094 & Cell Signaling \\
\hline BUB3 & Mouse/monoclonal & $1: 1000$ & sc-376506 & $\begin{array}{l}\text { Santa Cruz } \\
\text { Biotechnology }\end{array}$ \\
\hline MAD2L1 (D8A7) & Rabbit/monoclonal & $1: 1000$ & 4636 & Cell Signaling \\
\hline pVHL & Mouse/monoclonal & $1: 200$ & 556347 & BD Pharmingen \\
\hline Actin (C-11) & Goat/polyclonal & $1: 1000$ & sc- 1615 & $\begin{array}{l}\text { Santa Cruz } \\
\text { Biotechnology }\end{array}$ \\
\hline Kv10.1 & Rabbit/polyclonal & $1: 1500$ & 9391 & \\
\hline Normal Mouse IgG & Mouse & $\begin{array}{l}\text { Isotype } \\
\text { control }\end{array}$ & sc-2025 & $\begin{array}{l}\text { Santa Cruz } \\
\text { Biotechnology }\end{array}$ \\
\hline Normal Rabbit IgG & Rabbit & $\begin{array}{l}\text { Isotype } \\
\text { control }\end{array}$ & sc-3888 & $\begin{array}{l}\text { Santa Cruz } \\
\text { Biotechnology }\end{array}$ \\
\hline $\begin{array}{l}\text { ECL Anti-Rabbit IgG, } \\
\text { HRP Linked }\end{array}$ & Donkey/polyclonal & $1: 10000$ & NA934 & GE Healthcare \\
\hline $\begin{array}{l}\text { ECL Anti-Mouse IgG, } \\
\text { HRP Linked }\end{array}$ & Sheep/polyclonal & $1: 10000$ & NA931 & GE Healthcare \\
\hline $\begin{array}{l}\text { Anti-Goat IgG }(\mathrm{H}+\mathrm{L}) \text {, } \\
\text { HRP Conjugate }\end{array}$ & Rabbit/polyclonal & $1: 10000$ & $172-1034$ & BIO-RAD \\
\hline
\end{tabular}

\subsubsection{Immunoprecipitation}

Due to the low endogenous levels of Kv10.1, the efficiency of Kv10.1 knockdown in HeLa and hTERT RPE1 cells was tested by immunoprecipitation and WB. The samples were immunoprecipitated with protein G magnetic beads (New England BioLabs) following the manufacturer's instructions. In brief, $300 \mu$ l of cell lysate 
containing $1000 \mu \mathrm{g}$ of total protein was incubated with $15 \mu \mathrm{l}$ of protein $\mathrm{G}$ magnetic beads for $1 \mathrm{~h}$. Afterwards, the supernatant was recovered using a magnetic separation rack (New England Biolabs). This step pre-clears the sample from components that bind non-specifically to the magnetic beads. Then, $3 \mu \mathrm{g}$ of anti-Kv10.1 monoclonal antibody (Kv10.1-33.mAb) was added to and incubated with the pre-cleared samples on a vertical rotator at $4^{\circ} \mathrm{C}$, overnight. On the following day, the Kv10.1/antibody complexes were recovered by addition of $30 \mu \mathrm{l}$ of protein $\mathrm{G}$ magnetic beads per sample, and incubation for $4 \mathrm{~h}$ at $4^{\circ} \mathrm{C}$ on a vertical rotator. Afterwards, protein $\mathrm{G}$ magnetic beads/antibody/Kv10.1 complexes were pulled by placing the samples into a magnetic separation rack. The supernatant was removed and stored at $-20^{\circ} \mathrm{C}$.

The beads were washed 3 times with $500 \mu \mathrm{l}$ of cold Immunoprecipitation buffer $(0.1 \%$ Triton X-100, 50 mM Tris-HCl, $300 \mathrm{mM} \mathrm{NaCl,} 5 \mathrm{mM}$ EDTA) with protease inhibitor cocktail (Roche). Afterwards, the magnetic bead/antibody/Kv10.1 pellets were resuspended in $16.25 \mu \mathrm{l}$ TBS buffer, $2.5 \mu \mathrm{l}$ NuPAGE reducing agent (Invitrogen) and $6.25 \mu \mathrm{l}$ NuPAGE LDS Sample buffer (Invitrogen), and incubated for $10 \mathrm{~min}$ at $70^{\circ} \mathrm{C}$. The latter resulted in the denaturation of the antibody and Kv10.1 and disruption of the magnetic beads/antibody/Kv10.1 complexes. The protein samples were resolved on $4-12 \%$ NuPAGE Novex

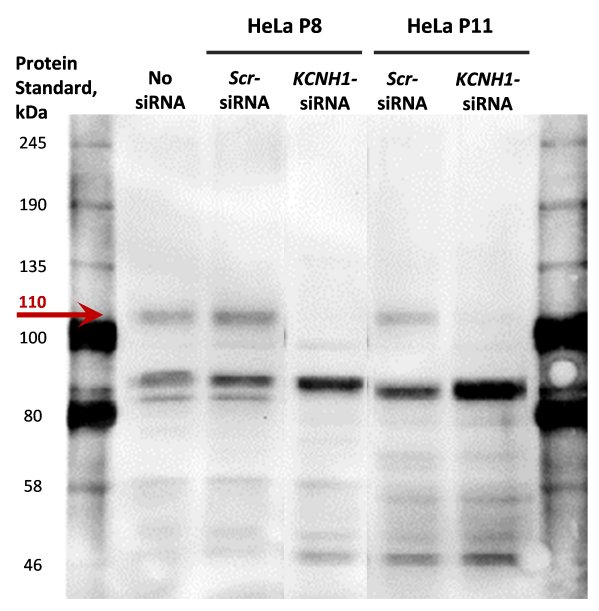

Figure 2.2: Kv10.1 silencing was defined by immunoprecipitation. An example image from two independent experimental repeats is given (the arrow indicates the Kv10.1 corresponding band).

Bis-Tris Mini Gels (Invitrogen) at $200 \mathrm{~V}$ and im-

munoblotted on $0.2 \mu \mathrm{m}$ NC membranes as described in sections "SDS-PAGE" and "Immunoblotting". An example of an immunoblot image from Kv10.1 immunoprecipitation is illustrated in Figure 2.2.

\subsubsection{Densitometry}

The changes in expression of the protein of interest were estimated by applying densitometry analysis to the acquired immunoblotting images. Careful consideration was given to the preparation of the samples in order to get a reliable end result. The 
densitometry analysis was performed on ImageLab software version 4.1 (Bio-Rad). Briefly, the "Lane and Bands" tool was used for selecting and defining each band on an immunoblotting image using the "Lane profile" intensity histogram. The background-subtraction was done using the rolling disk (size $8 \mathrm{~mm}$ ) tool of the software. The molecular weight was determined by interpolating the migration distance to that of the markers using the "MW" analysis tool. The normalised volume intensity of each signal was then calculated by dividing its measured volume intensity by the corresponding actin value.

$$
\text { Relative Intensity }=\frac{\text { Band Volume Intensity of the protein of interest }}{\text { Band Volume Intensity of a corresponding house-keeping protein }}
$$

\subsection{Single-cell gel electrophoresis}

One of the commonly used methods for detection of DNA lesions is single-cell gel electrophoresis, also known as comet assay. It is a microgel electrophoresis technique, which allows to quantify DNA damage on a single-cell level; the structures observed under the microscope have a resemblance to a comet, hence the alternative name (Neri et al., 2015). The alkaline variation of the method allows to detect SSBs, DSBs and alkaline labile sites (Singh, McCoy, Tice, \& Schneider, 1988; Speit \& Rothfuss, 2012). The sensitivity of the assay lies in a range of a

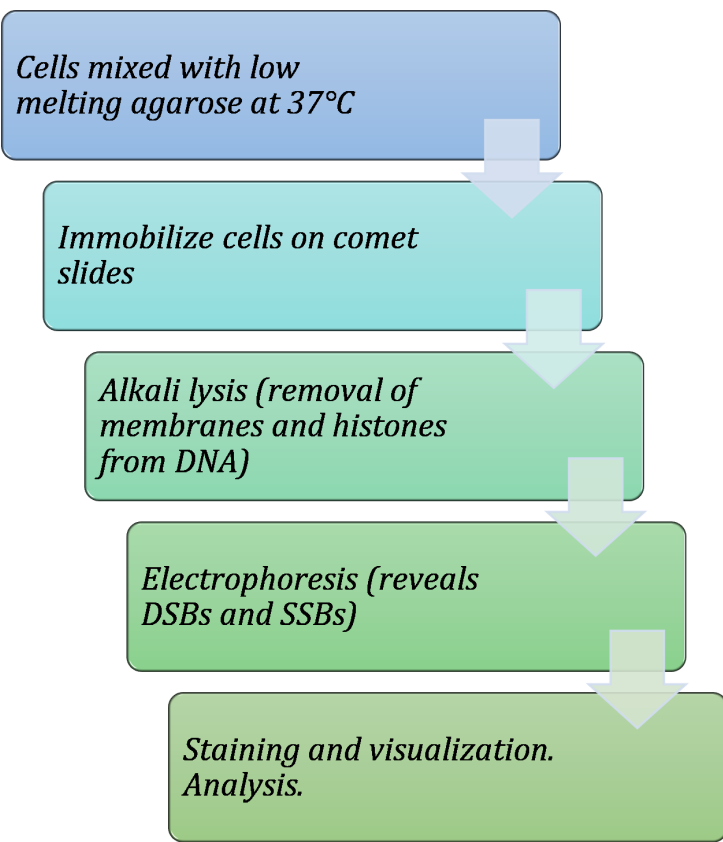

Figure 2.3: An outline of the steps of single-cell gel electrophoresis. few hundred to several thousand breaks per cell, thus low endogenous damage levels can also be distinguished (Langie, Azqueta, \& Collins, 2015).

The alkaline protocol described by A. Azqueta and A. Collins (2014) was used for detection of endogenously occurring DNA lesions in HeLa and hTERT RPE1 cells. HeLa and hTERT RPE1 cells were plated in a 6-well plate, and transfected either 
with Scr-or KCNH1-siRNA on the following day. The comet assay was performed 48 $\mathrm{h}$ after siRNA interference. The protocol is outlined in Figure 2.3, and the detailed description of each step follows. The entire procedure was carried out under dim light in order to prevent additional DNA damage, and all incubations, unless otherwise stated, at $4^{\circ} \mathrm{C}$, to prevent activation of the DNA repair mechanisms. The buffers and their compositions are given in Table 2.2.

Preparation of comets slides. The comet slides were prepared four days before the lysis step. CometSlide 2 Well (Trevigen; two slides per condition) were placed in ethanol at $-20^{\circ} \mathrm{C}$ for $24 \mathrm{~h}$ and then air dried overnight at RT. This step ensured the cleanliness of slides to facilitate the adhesion of an agarose layer on them. A $0.5 \%$ solution of the NMP (normal melting point) agarose was prepared by dissolving it in DPBS using a microwave. The NMP agarose solution was kept on a water bath, and the clean comet slides were submerged in it. The excess of NMP agarose was allowed to drain on a filter paper. Solidification of the agarose layer was done at $60-$ $65^{\circ} \mathrm{C}$ for $20 \mathrm{~min}$, and after that the slides were kept at RT until use.

Lysis. Prior to lysis, a $1 \%$ solution of LMP (low melting point) agarose in DPBS was prepared in the same way as the NPA solution and kept at $37^{\circ} \mathrm{C}$. The cells growing in a monolayer were washed once with cold DPBS, then harvested by trypsinisation and placed on ice. An aliquot of the cells (30000 cells) was then resuspended in $100 \mu \mathrm{l}$ cold DPBS, pipetted thrice using a blue tip, and immediately mixed with $100 \mu \mathrm{l}$ of LMP agarose. A $100 \mu \mathrm{l}$ aliquot of the cell-agarose mixture was spread onto a well of each comet slide and placed at $4^{\circ} \mathrm{C}$ for $20 \mathrm{~min}$ until the agarose layer was solid.

The slides were then carefully submerged into $50 \mathrm{ml}$ of lysis buffer (enough to cover the slides), and incubated for $1 \mathrm{~h}$. This step removes cell membranes, soluble cytoplasmic and nuclear components and renders supercoiled DNA.

Denaturation, electrophoresis, neutralisation and fixation. Comet assay results can vary depending on temperature, the distance between electrodes, and buffer height in the tank during electrophoresis. Hence, standardisation of each of those parameters is required to increase reproducibility.

Immediately after the lysis, the slides were placed side by side and in the same direction onto a levelled electrophoresis tank filled with $280 \mathrm{ml}$ of cold electrophoresis 
Table 2.2: Composition of buffers used in the comet assay

\begin{tabular}{|c|c|c|}
\hline Step in the protocol & Name of the solution & Composition \\
\hline \multirow{10}{*}{$\begin{array}{l}\text { Pre-coating, embedding and } \\
\text { lysis }\end{array}$} & \multirow{2}{*}{$\begin{array}{l}\text { Agarose solution for pre-coating of } \\
\text { the slides }\end{array}$} & $1 \%$ NMP agarose in DPBS; \\
\hline & & aliquoted and stored at $4^{\circ} \mathrm{C}$ \\
\hline & \multirow{2}{*}{ Agarose for embedding the cells } & 1\% LMP agarose in DPBS; \\
\hline & & aliquoted and stored at $4^{\circ} \mathrm{C}$ \\
\hline & \multirow{6}{*}{ Lysis buffer } & $2.5 \mathrm{M} \mathrm{NaCl}$ \\
\hline & & $0.1 \mathrm{M}$ EDTA \\
\hline & & $10 \mathrm{mM}$ Tris \\
\hline & & $10 \%$ DMSO \\
\hline & & $\begin{array}{l}1 \% \text { Triton } \mathrm{X}-100 \text { (added after) } \\
\mathrm{pH}=10, \text { in } \mathrm{ddH}_{2} \mathrm{O}\end{array}$ \\
\hline & & stored at $4^{\circ} \mathrm{C}$ \\
\hline \multirow{6}{*}{$\begin{array}{l}\text { Denaturation, electrophoresis } \\
\text { and neutralisation }\end{array}$} & \multirow{4}{*}{ Electrophoresis buffer } & $1 \mathrm{mM} \mathrm{Na}_{2} \mathrm{EDTA}$ \\
\hline & & $300 \mathrm{mM} \mathrm{NaOH}$ \\
\hline & & $\mathrm{pH}>13$, in $\mathrm{ddH}_{2} \mathrm{O}$ \\
\hline & & stored at $4^{\circ} \mathrm{C}$ \\
\hline & \multirow[t]{2}{*}{ Neutralisation buffer } & $\begin{array}{l}0.4 \mathrm{M} \text { Tris, } \\
\mathrm{pH}=7.5, \text { in } \mathrm{ddH}_{2} \mathrm{O}\end{array}$ \\
\hline & & stored at $4^{\circ} \mathrm{C}$ \\
\hline \multirow{3}{*}{ Fixation and staining } & Fixation solution & $100 \%$ ethanol \\
\hline & \multirow[t]{2}{*}{ Staining solution } & $\begin{array}{l}1 \times \text { SYBR Gold } \\
10 \mathrm{mM} \text { Tris- } \mathrm{HCl}, \mathrm{pH}=8.0 \\
1 \mathrm{mM} \text { EDTA }\end{array}$ \\
\hline & & prepared right before use \\
\hline
\end{tabular}

buffer. The samples were denatured with the electrophoresis buffer for $20 \mathrm{~min}$. Denaturing of supercoiled DNA is critical for unveiling the lesion sites: the break containing sites will be relaxed and migrate easier when the electric field is applied. The electrophoresis was carried out for $20 \mathrm{~min}$ at $13 \mathrm{~V}(0.6 \mathrm{~V} / \mathrm{cm}$ across the platform) and $300 \mathrm{~mA}$. The amperage was adjusted by adding or removing buffer (if needed). In this step, if the DNA contains a lesion it will migrate faster depending on the size of the DNA strand breaks.

After the electrophoresis the slides were placed into a Coplin jar with neutralisation buffer ( $4 \mathrm{ml} /$ slide) for $10 \mathrm{~min}$, and then fixed by incubating in absolute ethanol for $3 \mathrm{~min}$. The fixed slides were then air dried at RT in the dark.

Staining and imaging. The slides were stained using SYBR Gold (Invitrogen) following the manufacturer's instructions. An aliquot of SYBR Gold was diluted 10000 times in TE buffer.

The air dried comet slides were incubated with the dye for $10 \mathrm{~min}$ at RT, and immediately imaged on a Nikon TiE-Andor inverted microscope (Nikon) equipped 
with a PLAN APO VC Air 20x 0.75 N.A. (numerical aperture) objective, a Yokogawa spinning disk confocal. SYBR Gold was excited with the $488 \mathrm{~nm}$ laser line and emission was collected through a 525/30 emission filter. Images were acquired with an Andor DU-888 X-9984 camera controlled with NIS-Elements software. In total, 10 positions per well were selected using the multipoint acquisition of NIS-Elements software. A series of z-stack images with $0.2 \mu \mathrm{m}$ z-stepsize were acquired. An exposure time of $50 \mathrm{~ms}$ (optimal for the chosen settings), no binning and no illumination between acquisitions was used in all experiments.

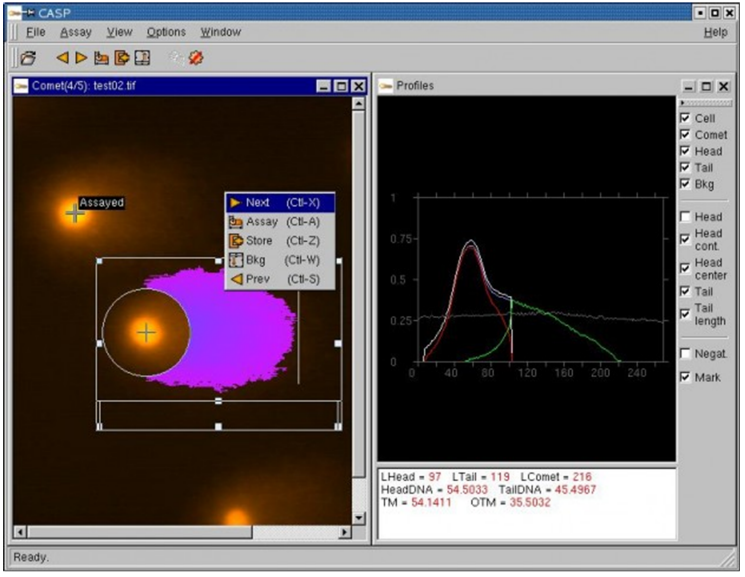

Figure 2.4: A screenshot of a CASP Lab software showing the measurement window and an assayed comet with the corresponding intensity profile in "Profiles"window.
Analysis. The damaged DNA has a higher mobility and migrates further when electric field is applied, creating a configuration similar to a comet (Speit \& Rothfuss, 2012). The head of the comet corresponds to a circle comprising the non-damaged DNA, and the tail contains the migrated damaged DNA. Measuring the intensity of the head and tail, as well as the length of the tail provides information on the extent of DNA damage present in the samples.

Before the analysis, maximum intensity projection images were created from the acquired z-stack images. Then, the DNA comets were analysed using CASPLab open source software. The program works with either colour or greyscale images of fluorescently labelled comets saved as a TIFF (tagged image format file), and only comets oriented with the head on the left and the tail on the right can be analysed correctly (Końca et al., 2003).

In brief, two frames adjacent to each other are defined, one containing a test comet, and the other one corresponding to background pixel values. Once the sizes of the measurement frames are defined and fixed, the same size will be used throughout the analysis. The measurement consists on the generation of an intensity profile Figure 2.4) from which the comet total length, head radius, tail length, DNA intensity in the head and tail, percentage of DNA in the head and tail, TMo (tail moment) and 
Table 2.3: Result parameters and modes of measurement used by CASP Lab software (Końca, 2015)

\begin{tabular}{ll}
\hline Name of parameter & Explanation \\
Name & Name of the image file \\
HeadArea & Area of the comet head in pixels (sum of pixels in the head) \\
TailArea & Area of the comet tail in pixels (sum of pixels in the head) \\
TailDNA & Amount of DNA in the comet head (sum of intensities of pixels in the head) \\
HeadDNA\% & Amount of DNA in the comet tail (sum of intensities of pixels in the tail) \\
TailDNA\% & Per cent of DNA in the comet head \\
HeadRadius & Per cent of DNA in the comet tail \\
TailLength & The radius of the comet head (in pixels) \\
CometLength & Length of the comet tail measured from the right border of head area to end of the \\
tail (in pixels) \\
HeadMeanX & $\begin{array}{l}\text { Length of the entire comet from the left border of the head area to end of the tail } \\
\text { (in pixels) }\end{array}$ \\
TailMoment & Centre of gravity of DNA in the head (x coordinate) \\
Olive TailMoment & Centre of gravity of DNA in the tail (x coordinate) \\
& TailDNA\% * TailLength ([per cent of DNA in the tail] * [tail length]) \\
& TailDNA\% between the centre of gravity of DNA in the tail and the of the centre of gravity of \\
\hline
\end{tabular}

OTM (Olive tail moment; Table 2.3). In general, the different ways of measurement tail moments aim to integrate the information on the shape, size and intensity of the comet. The TMo considers only the features of the comet tail. It is derived by multiplying the tail length with tail intensity and divided by 100 . The OTM takes into consideration the entire shape of the comet and is the product of tail DNA multiplied by the difference between the gravity centres of the comet head and comet tail and divided by 100 (Azqueta \& Collins, 2014; Langie et al., 2015; Sierra \& Gaivão, 2014). The already analysed comet can be marked as "assayed", thus preventing accidental re-measurement of the same comet. Multiple cells per image or images can be processed. Once all the loaded images have been assayed, the results are exported as a .txt file.

\subsection{Immunofluorescence staining}

Asymmetric mitotic spindles, misaligned chromosomes at metaphase and lagging chromosomes at anaphase were assessed by immunofluorescence staining of mitotic spindles ( $\alpha$-tubulin), centromeres (CENP-B, centromere protein B) and DNA (Hoechst 33342). The mitotic spindle angle was determined by additionally staining the centrosomes ( $\gamma$-tubulin).

HeLa cells were plated on fibronectin (Sigma) coated coverslips (Menzel) in a 
6-well plate (Greiner) and synchronised according to the protocols described above. For the experiments requiring preservation of MTs, the growth medium was aspirated, and the cells were first fixed by incubating with $4 \%$ paraformaldehyde (pfa) solution in PHEM buffer (60 mM PIPES, 27 mM HEPES, 10 mM EGTA, 4 mM MgSO4, $\mathrm{pH}=7.0)$ at $\mathrm{RT}$ for 5 min. Afterwards, the pfa solution was replaced by ice-cold methanol and incubated at $-20^{\circ} \mathrm{C}$ for additional $5 \mathrm{~min}$. The fixed cells were then washed three times with DPBS, and stored at $4^{\circ} \mathrm{C}$ until usage. For proximity ligation assay, the cells were only fixed with $4 \%$ pfa in PHEM, washed and stored as described above.

Prior to staining, the fixed cells were rinsed with DPBST (0.1\% Tween 20; Sigma) and blocked with 10\% BSA (Sigma) in DPBST for 90 min, to reduce non-specific binding of antibodies. Primary and secondary antibodies were diluted in the blocking solution according to Table 2.4.

Afterwards, the coverslips were transferred with the cells facing down onto droplets of primary antibodies placed on a Parafilm (Sigma-Aldrich) tape in a humidified chamber and incubated for $2 \mathrm{~h}$ at RT, and then the cells were incubated with secondary antibodies for $1 \mathrm{~h}$ using the same approach, although in the dark to prevent photobleaching of fluorescently labelled secondary antibodies. Unbound primary and secondary antibodies were eliminated by washing three times with PBST for 5 min at RT with gentle agitation. At last, the nuclei were counterstained with $10 \mu \mathrm{M}$ Hoechst 33342 (Molecular Probes) in $\mathrm{ddH}_{2} \mathrm{O}$ and rinsed twice with $\mathrm{ddH}_{2} \mathrm{O}$. The coverslips with immunofluorescently-labelled cells were air dried and mounted onto microscope slides (Thermo Scientific) using Prolong Gold antifade reagent (Invitrogen). The

Table 2.4: List of primary and secondary antibodies used for immunoblotting

\begin{tabular}{|c|c|c|c|c|}
\hline Antibody & Source & Dilution & $\begin{array}{l}\text { Cat. } \\
\text { Number }\end{array}$ & Manufacturer \\
\hline$\gamma$-Tubulin & Goat/polyclonal & $1: 350$ & sc-7396 & $\begin{array}{l}\text { Santa Cruz } \\
\text { Biotechnology }\end{array}$ \\
\hline$\alpha$-Tubulin & Mouse/monoclonal & $1: 700$ & sc-23948 & $\begin{array}{l}\text { Santa Cruz } \\
\text { Biotechnology }\end{array}$ \\
\hline CENP-B (H-65) & Rabbit/polyclonal & $1: 350$ & sc-22788 & $\begin{array}{l}\text { Santa Cruz } \\
\text { Biotechnology }\end{array}$ \\
\hline $\begin{array}{l}\text { Alexa Fluor } 488 \text { anti-mouse } \\
\text { IgG }(H+L)\end{array}$ & Donkey/polyclonal & $1: 1000$ & A-21202 & Invitrogen \\
\hline $\begin{array}{l}\text { Alexa Fluor } 546 \text { anti-rabbit } \\
\text { IgG }(H+L)\end{array}$ & Donkey/polyclonal & $1: 1000$ & A-11056 & Invitrogen \\
\hline
\end{tabular}


mounting medium was allowed to polymerise overnight, and on the following day the slides were visualised on a Leica TCS SP5 II confocal laser scanning microscope (Leica). Images were acquired using LAS AF (Leica) software, and processed with the image analysis software ImageJ2 (NIH; Collins, 2007; Rueden et al., 2017; Schneider, Rasband, \& Eliceiri, 2012) and Imaris (Bitplane).

\subsubsection{Quantification of asymmetric mitotic spindles, defective metaphases and lagging chromosomes}

A monopolar mitotic spindle usually displays symmetric configuration, meaning that the length of MTs is equal in all directions, otherwise the monopolar mitotic spindle will be qualified as asymmetric (Figure 2.5, A).

Metaphases were considered defective when improper alignment of chromosomes at the metaphase plate was observed (Figure 2.5, B). A lagging chromosome was defined as an event positive for both CENP-B and Hoechst 33342 staining, located in the equatorial region of an anaphase cell (Figure 2.5, C). Using a confocal fluorescent microscope, in total 1500 cells per condition and experiment were counted manually. Cells with asymmetric mitotic spindles, misaligned chromosomes and lagging chromosomes were expressed as a percentage of the total number of cells.

\subsubsection{Determination of mitotic spindle angle}

Mitotic spindle angle was measured in HeLa cells synchronised at metaphase and visualised as described above. The obtained images were further processed with Imaris (Bitplane). The processing of the images is depicted in Figure 2.6. In brief, the location of centrosomes was defined using the measurement points tool, choosing the channel corresponding to the centrosomal marker used for the staining (red in this case). The software automatically finds intensity maxima of the selected channel in the area specified by the user. Three measurement points were created, defined as a sphere with a diameter of $1.5 \mu \mathrm{m}$ around the maximum. Two of the points were located at the centrosomes as shown in Figure 2.6 as point "A" and "B". The third point, "C" was generated as the projection of centrosome A at the level of centrosome $\mathrm{B}$, that is, at $(\mathrm{x}, \mathrm{y})$ coordinates of centrosome $\mathrm{A}$, and $\mathrm{z}$-coordinate of centrosome $\mathrm{B}$. 

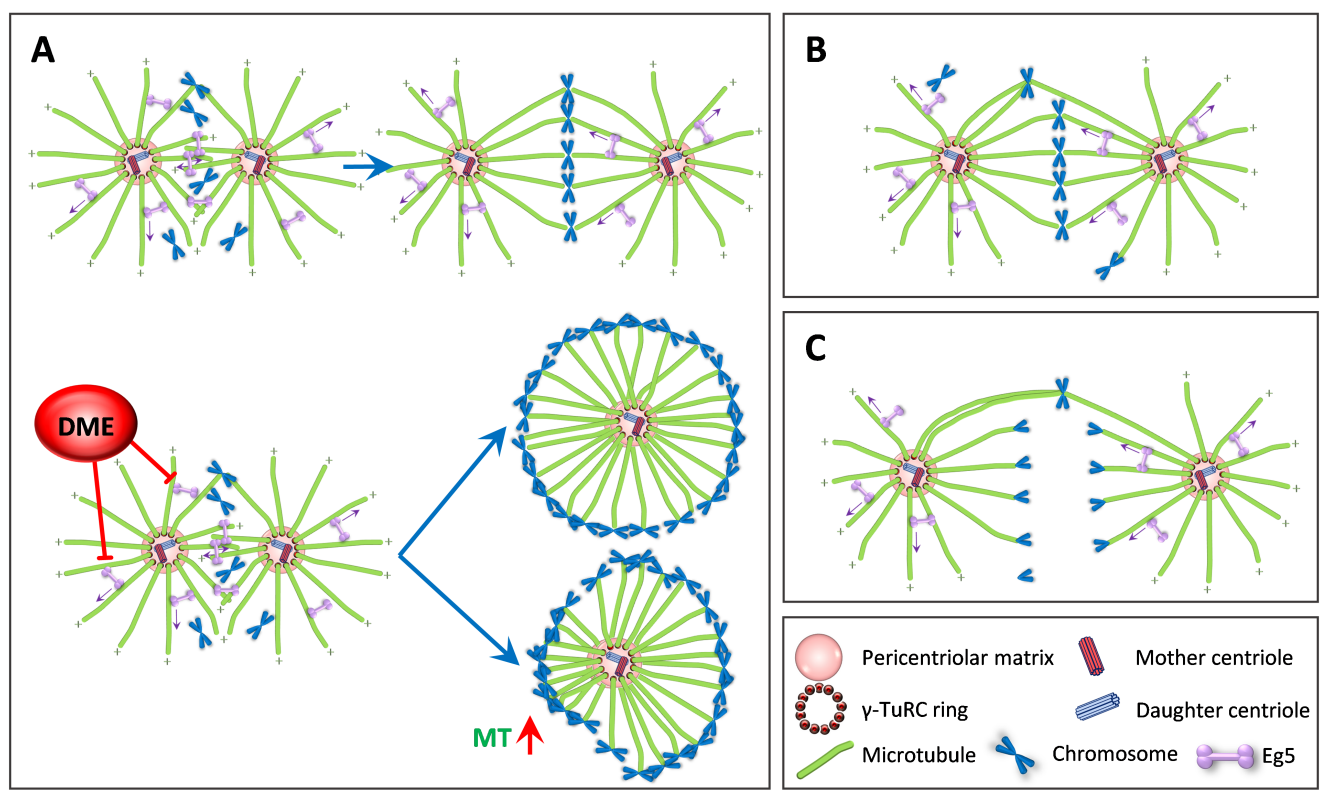

Figure 2.5: Schematic representation of some mitotic structures. The mechanism of $D M E$ action is explained. Inhibition of Eg5 motor protein leads to a formation of monopolar mitotic spindles, which are symmetric, unless MT plus-end assembly rates are increased. An example of a metaphase with misaligned chromosomes $(B)$ and an anaphase with a lagging chromosome are presented.

Then, the polygon line mode was selected, which yielded three readouts: the distance between "A" and "B" (the two centrosomes), distance between "B" and "C" (the centrosome one and the projection of centrosome two), and the angle between the two distance lines. The distance between "A" and " $\mathrm{B}$ " is the interpolar distance, and the angle is the mitotic spindle angle. The measurements were done on at least 50 cells per condition and experiment.

\subsection{Determination of PLK1 enzymatic activity}

Polo-like kinase 1 activity was quantitatively measured in cell lysates from HeLa and hTERT RPE1 cells using CycLex Polo-like kinase 1 Assay/Inhibitor Screening Kit (Cyclex, MBL).

Before starting with the assay, all the components of the kit were allowed to come to RT. The 1x Wash Buffer was prepared by diluting the 10x Wash Buffer (contains Tween-20; CycLex Kit) with $\mathrm{ddH}_{2} \mathrm{O}$, and a $1.25 \mathrm{mM}$ ATP solution - by dissolving the lyophilised 20x ATP (contains sodium salts, CycLex Kit) in $1.6 \mathrm{ml}$ of $\mathrm{ddH}_{2} \mathrm{O}$. Afterwards, the Kinase Reaction Buffer was prepared by adding $10 \mu \mathrm{l}$ of $1.25 \mathrm{mM}$ ATP solution to $190 \mu$ l of Kinase Buffer (CycLex Kit). 

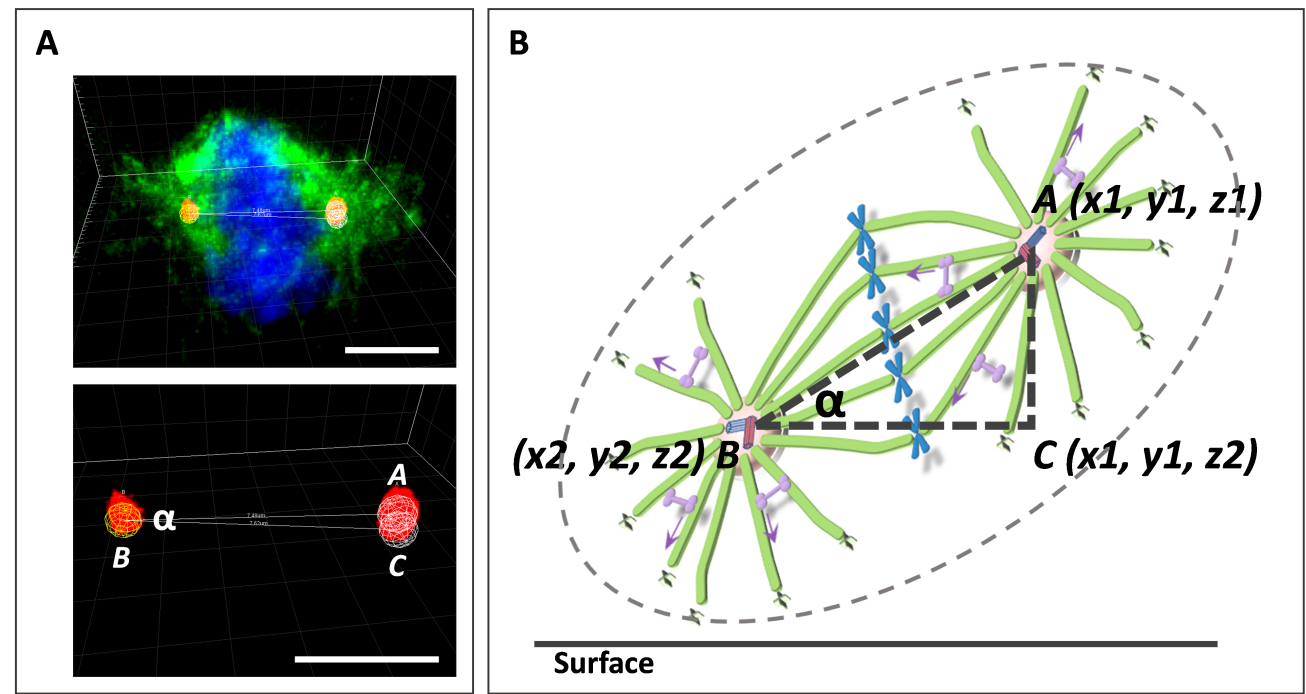

Figure 2.6: Determination of a mitotic spindle angle. A, Screenshots of the measurement using Imaris is given (for the details, see the text; in red - $\gamma$-tubulin, green $-\alpha$-tubulin, blue - Hoechst33342; scale bar, $4 \mu \mathrm{m})$. B, The approach for MT angle measurement is explained schematically.

Once all working solutions were ready, the lysates from synchronised HeLa and hTERT RPE1 cells were retrieved from $-80^{\circ} \mathrm{C}$, and $20 \mu \mathrm{l}$ of lysate (see "Protein extraction") containing $50 \mu \mathrm{g}$ of protein was added to each microtiter well coated with recombinant Protein-X as a PLK1 substrate (CycLex Kit). The microtiter wells were kept on ice between all incubation steps. The reaction was started by addition of $180 \mu \mathrm{l}$ of Kinase Reaction Buffer, and the samples were incubated for $2 \mathrm{~h}$ at $30^{\circ} \mathrm{C}$. The reaction was stopped by removing the cell lysates from the well; immediately $200 \mu \mathrm{l}$ of $1 \mathrm{x}$ Wash Buffer was added, and the well content was again aspirated. The washes were repeated 5 times, followed by the addition of $100 \mu \mathrm{l}$ of anti-phosphothreonine antibody (PPT-07, rabbit polyclonal, CycLex Kit) to each microtiter well and incubated for $1 \mathrm{~h}$ at $30^{\circ} \mathrm{C}$. If the samples contain an active PLK1, then the Protein-X substrate will be phosphorylated at its threonine residue, which will be then recognised by the primary antibody. Afterwards, the wells were washed as described, and incubated with $100 \mu \mathrm{l}$ of HRP-conjugated anti-rabbit antibody (CycLex Kit) for $30 \mathrm{~min}$ at $30^{\circ} \mathrm{C}$. Again, the wells were washed as before, and $100 \mu \mathrm{l}$ of chromogenic Substrate Reagent (CycLex Kit) was added per well and incubated for $10 \mathrm{~min}$ at RT, protected from light. The chromogenic reaction was stopped by addition of $100 \mu \mathrm{l}$ of Stop Solution (CycLex Kit). The absorbance in each well 
was measured using dual wavelengths at 450/540 nm on a Wallac 1420 VICTOR2 microplate reader (PerkinElmer, Inc., USA).

As a positive control, $1 \mu \mathrm{l}$ of purified recombinant PLK1 (0.004 units/ $\mu$; human full-length recombinant enzyme expressed in E. Coli; CycLex, MBL) in $19 \mu$ of the lysis buffer was used. As a negative control only the lysis buffer was used. The lysis buffer composition is given in "Protein extraction". All samples were prepared in duplicates.

The difference of absorbances at $450 \mathrm{~nm}$ and $540 \mathrm{~nm}$ was calculated for each sample. Then the absorbance of each sample was normalised against the negative control.

$$
A 450=\left(A 450_{\text {sample }}-A 540_{\text {sample }}\right)-\left(A 450_{\text {neg ctr }}-A 540_{\text {neg ctr }}\right)
$$

\subsection{Proximity ligation assay}

The possible interaction of Kv10.1 and ORAI1 was studied by means of PLA (proximity ligation assay). The proximity ligation method relies on the formation and amplification of DNA circles catalysed by RCA (rolling circle amplification) when antibodies labelled with compatible oligonucleotides bind to targets located in proximity (Söderberg et al., 2006).

The method was initially developed by Fredriksson et al. in 2002. They applied the principle of polymerase chain reaction for the detection of protein targets and termed it proximity ligation. Initially, pairs of affinity probes, such as DNA aptamers, simultaneously recognise the target molecules in proximity, generating an intensified signal (Fredriksson et al., 2002). Later, the technique was generalised by providing easy protocols to create proximity probes from any poly- or monoclonal antibodies (Gullberg et al., 2004). Finally, the PLA was further modified by combining it with RCA for localised readout in fixed samples. This step in combination with fluorescently labelled oligonucleotides generates multiple DNA circles, covalently bound to an antibody-antigen complex, and, thus, making them detectable (Söderberg et al., 2006).

The Duolink PLA kit (Sigma-Aldrich) was used for the detection of interaction of Kv10.1 and ORAI1 in asynchronously growing HeLa cells following manufacturer's 
instructions. The cells growing in a 6-well plate on fibronectin-coated coverslips were fixed (described in "Immunofluorescence staining"), and an area of approximately $1 \mathrm{~cm}^{2}$ was delimited by a hydrophobic pen. A drop of Duolink Blocking Solution was deposited onto the defined area, and the coverslips were then transferred into a heated humidity chamber at $37^{\circ} \mathrm{C}$ and incubated for 60 min. Meanwhile, a diluted aliquot (40 $\mu \mathrm{l} /$ sample) of a mixture of primary antibodies was prepared using Duolink Antibody Diluent: anti-Kv10.1 (mAb62; 1:2000, mouse monoclonal) and anti-ORAI1 (Alomone biolabs; 1:200, rabbit polyclonal). The blocking solution was gently tapped off the samples, and an aliquot of the primary antibody mixture was added to each sample, and then incubated for $3 \mathrm{~h}$ at RT. Samples treated with the only diluent were used as a negative control for the assay. Afterwards, the antibody solutions were removed, and the slides were washed twice for 5 min in 1x Wash Buffer A (Duolink kit) at RT. During the washes, the PLUS and MINUS PLA probes were vortexed and diluted 5 times with Duolink Antibody Diluent. Again, the excess of wash buffer was removed, and $40 \mu \mathrm{l}$ of the mixture with PLUS and MINUS probes were added to all samples, including the negative control. The PLA probe incubation was performed for $60 \mathrm{~min}$ in a humidity chamber at $37^{\circ} \mathrm{C}$. Following the incubation with PLA probes, the samples were washed as above and incubated with $40 \mu \mathrm{l} /$ sample Ligase solution (prepared from $1 \mu$ l Ligase and $8 \mu \mathrm{l}$ x Ligation buffer) for $30 \mathrm{~min}$ at $37^{\circ} \mathrm{C}$ in a humidity chamber. Similarly, the ligase solution was removed, and the samples were washed twice with Wash buffer A. From this point on, the steps are light sensitive and were performed under dim light. The amplification solution was prepared by adding $0.5 \mu \mathrm{l}$ of Polymerase to $39.5 \mu \mathrm{l}$ of Amplification buffer. The samples were incubated with $40 \mu \mathrm{l}$ of amplification solution for $100 \mathrm{~min}$ at $37^{\circ} \mathrm{C}$ in a humidity chamber. The excess of amplification solution was tapped off the samples, and the samples were washed twice with 1x Wash Buffer B (Duolink kit) for 10 min, and once with 0.01x Wash Buffer B for 1 min. Finally, the excess of wash buffer was removed as before and the coverslips were mounted onto slides with a minimal volume of Duolink PLA Mounting Medium with DAPI. The imaging was done as described in the "Immunofluorescence staining" section, and after that, the slides were stored at $4^{\circ} \mathrm{C}$ for a short period (up to 4 days) or at $-20^{\circ} \mathrm{C}$ for extended periods. 


\subsection{Live cell imaging}

All time-lapse imaging was performed on a Nikon TiE-Andor inverted microscope (Nikon) equipped with a PLAN APO-TIRF 100x 1.49 N.A. oil immersion objective, a Yokogawa spinning disk confocal system and the Perfect Focus System (Nikon) for continuous maintenance of the focal plane. Fluorophores were excited with the respective laser line from a MultiLaser (Acal), and the emitted light was collected through a quadruple bandpass dichroic mirror and the appropriate emission filter. Images were acquired with an Andor DU-888 X-9984 camera controlled with NISElements software. No binning and no illumination between acquisitions was used throughout all live cell imaging experiments. Exposure time, interval and duration were defined and maintained constant for each time-lapse experiment.

\subsubsection{Assessment of microtubule dynamics}

\section{Tracking of EB3-tdTomato in live cells}

MT plus-end assembly rates were determined by overexpressing and tracking EB3 fused to tdTomato (an orange fluorescent protein) in living HeLa and hTERT RPE1 cells. EB3 is an MT plus-end interacting protein (Galjart, 2010), and fluorescently tagged EB3 allows to identify the growing tips of MTs in living cells. Thus, by taking time-lapse images it is possible to measure the assembly and disassembly rates of MTs (reviewed in Zwetsloot, Tut, \& Straube, 2018).

HeLa and hTERT RPE1 cells were transfected with pEB3-tdTomato as described before. To ensure measurements of mitotic spindles in the same mitotic phase, 48 h after transfection 2.5 $\mathrm{MM}$ DME (Calbiochem) was applied to the cells growing in phenol red free DMEM medium (Gibco) supplemented with 10\% FCS and 1x GlutaMAX (Gibco). After $3.5 \mathrm{~h}$ of incubation with DME, the cells were transferred to the microscope and mounted on an environmental chamber with dark panels (Okolab) for imaging at $37^{\circ} \mathrm{C}$ and $5 \% \mathrm{CO}_{2}$, and further incubated until total incubation time of $4 \mathrm{~h}$. The maintenance of temperature at $37^{\circ} \mathrm{C}$ without fluctuations is crucial for the experiment. It has been described that the MT assembly rates depend on the ambient temperature (Gierke, Kumar, \& Wittmann, 2010; Lodish, 2000). Images were acquired every $0.5 \mathrm{~s}$ for $1 \mathrm{~min}$ as described above. The EB3-tdTomato was 
excited using the $561 \mathrm{~nm}$ laser line (exposure time $200 \mathrm{~ms}$ ), and the emission was collected with a 600/52 emission filter.

The MT dynamics were studied under five different conditions.

i) siRNA mediated downregulation of Kv10.1 - a co-transfection of EB3-tdTomato along with $S c r$ - or KCNH1-siRNA was performed (see "pDNA and siRNA co-transfection with jetPRIME");

ii) overexpression of Kv10.1 - pKCNH1-WT (wild type) or pEmpty were overexpressed simultaneously with pEB3-tdTomato in HeLa cells using jetPRIME (see "pDNA transfection using jetPRIME");

iii) reduction of Kv10.1 conductance by an inhibitory monoclonal antibody targeting its pore domain - HeLa cells were transfected with pEB3-tdTomato, and on the following day the cells were incubated with either mAb56 or a non-specific isotype $\operatorname{IgG} \kappa 2 \mathrm{~b} ; 5 \mu \mathrm{g}$ per well of a 4 -well $\mu$-ibidi dish) for $24 \mathrm{~h}$ at $37^{\circ} \mathrm{C}$ and $5 \%$ $\mathrm{CO}_{2}$. The last $4 \mathrm{~h}$-segment of the incubation with antibody was done in the presence of DME prior to imaging;

iv) inhibition of Kv10.1 conductance with the antihistamine drug astemizole (GarcíaFerreiro et al., 2004a), a potent blocker of channels of the KCNH family - HeLa cells expressing EB3-tdTomato were treated simultaneously with $2.5 \mu \mathrm{M}$ DME and $5 \mu \mathrm{M}$ astemizole or its isomer norastemizole, which retains antihistamine activity but has no channel inhibitory activity, or only combination of DME and the solvent (DMSO), and incubated for $4 \mathrm{~h}$ at $37^{\circ} \mathrm{C}$ and $5 \% \mathrm{CO}_{2}$;

v) AnCoA4-mediated inhibition of calcium influx through ORAI1 (Sadaghiani et al., 2014) - the treatment was done similarly to astemizole/norastemizole; EB3tdTomato expressing HeLa cells were incubated for $4 \mathrm{~h}$ with $10 \mu \mathrm{M}$ AnCoA4 or with the equivalent volume of the vehicle, DMSO, in the presence of $2.5 \mu \mathrm{M}$ DME.

\section{Analysis of the time-lapse series}

The MT dynamics were analysed with U-Track 2.2.0, a MATLAB based open source software. This software package automatically tracks, analyses, and visualises 
the time-lapse series of fluorescently-labelled MT plus-ends.

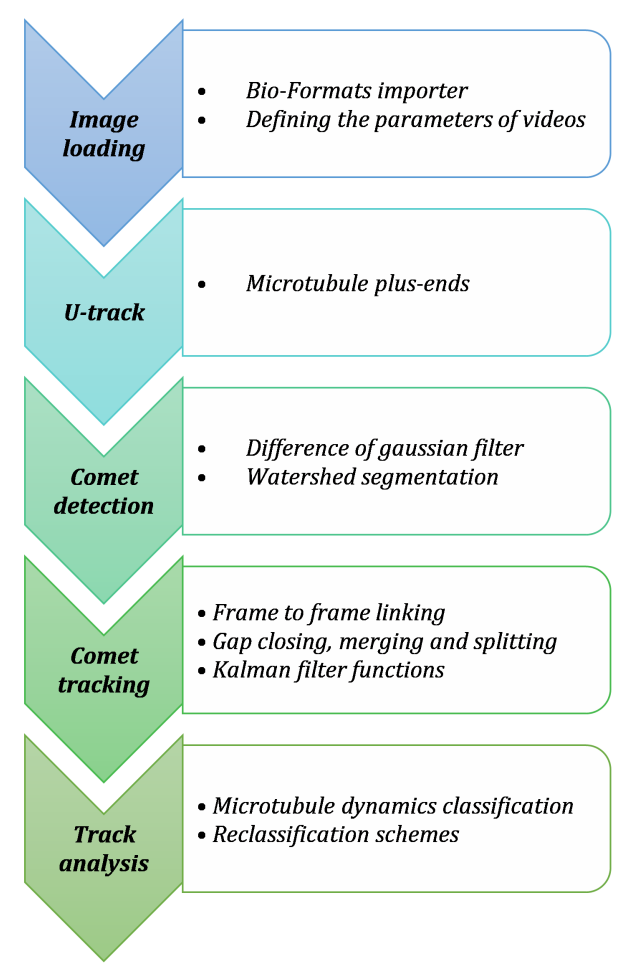

Figure 2.\%: An overview of the Track. $M T$ dynamics analysis using $U_{-}$

Despite that the plus-end-binding proteins trace only MT growth phases, the U-Track 2.2.0 extrapolates the information on the pause and shrinkage events by linking sequential growth phases meeting certain criteria. The reconstruction of full trajectories (Applegate et al., 2011; Matov et al., 2010) is based on the spatially and temporally global tracking framework (Jaqaman et al., 2008).

The analysis workflow is shown in Figure 2.7. First of all, the .nd2 files generated by NISElements software were imported into MATLAB. The size of each image was read from the metadata automatically, while the numerical aperture of the used objective (1.49) and the time interval (0.5 s) used for imaging had to be entered manually. Additionally, information on the excitation wavelength and the fluorophore can be entered. However they are not critical for further analysis.

Once all the images were loaded, MT tracking was performed using U-Track control. The analysis consists of three main steps: comet detection, comet tracking and track analysis. Each of these steps produces statistical and visual outputs. Below follows the brief description of each parameter and their functions; a more detailed explanation can be found in the technical report provided with the previous version of the software, plusTipTracker (Applegate \& Danuser, 2010).

Comet detection. The acquired signal from EB3-tdTomato appears as nearresolution sized comets, which vary in size, shape, and intensity over time and across regions of the cell. Image background signal also varies, and this together with the high density of the growing MTs and low signal-to-noise ratio causes that comets are often difficult to distinguish from the background and adjacent comets, precluding the use of a global detection threshold. This problem is circumvented by a 

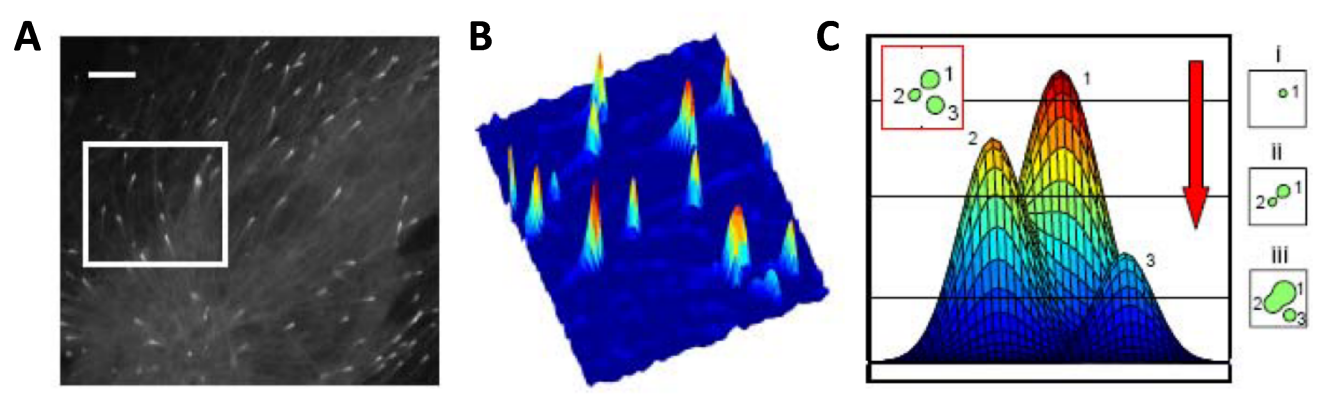

Figure 2.8: The watershed-based method for particle detection in U-Track. A, An non-processed image of EGFP-EB3-labelled MTs (scale bar, 5 $\mathrm{m}$ ). B, A Difference-of-Gaussian image is created from the original image and the intensity landscape is given. $C$, Schematic representation of an intensity landscape explaining the watershed particle detection method. The image is iterated with defined threshold, in this case thrice and is indicated with the horizontal lines. At each step the peaks are identified and shown in panels (i), (ii), and (iii). The stepwise thresholding distinguishes the peaks which otherwise would have been misdefined if a regular threshold approach was applied. Adapted from (Applegate 8 Danuser, 2010).

combination of image signal enhancement and locally optimal thresholds. The signal enhancement is achieved by the DoG (Difference of Gaussians) approach (Spring, K.R., J.C. Russ, M.J. Parry-Hill, T.J. Fellers, 2016). The DoG image is created by filtering the raw images first with a small Gaussian kernel ( $\sigma=1$ pixel), which eliminates the noise. Then, a larger Gaussian kernel ( $\sigma=3$ pixels) is applied, which removes larger-scale variations in cell background. The high-pass filtered image is then subtracted from the low-pass filtered image, resulting in DoG images with relatively uniform background and less noise (Figure 2.8, A).

The detection of comets is tuned by defining the size of the particles, which still vary significantly in size, shape, and intensity. For accurate identification of comets, it is necessary to maintain the expression of the fluorescently tagged protein at low levels, but this implies low intensity of the signal, yet high density of the comets, and a higher risk of false positive comets detection. To avoid this, a watershed particle segmentation approach is applied (Figure 2.8, B and C).

The watershed method is based on presenting a two-dimensional image as a threedimensional intensity landscape, which is then iteratively segmented with defined thresholds (Figure 2.8, C). Two parameters are defined: the minimum threshold and the threshold stepsize. The minimum threshold allows discerning weak signals from the background, while the background noise fluctuations will not be false positively accepted as comets. The threshold stepsize defines the accuracy of the comet detection: it should be small enough in order to resolve two particles with 
close intensity values from each other, but high enough to avoid having false positive comets. These parameters are derived from the intensity standard deviation of a DoG image: for each image $i$, the standard deviations from frames $i-2$ to $i+2$ are averaged to yield its threshold stepsize. The minimum threshold for each image is defined as three times the threshold step (Applegate \& Danuser, 2010). The defined segmentation steps are applied to the image collection, and thus the comets are identified.

Tracking comets. Comet trajectories over time are reconstructed according to the single-particle tracking framework described by Jaqaman et al. (2008). The tracking consists of two major steps: i) linking particle (generates sub-tracks) in consecutive frames and ii) linking the generated sub-tracks (compound tracks).

Particle linking picks the best set of links between particles, taking into account the entire population of particles in two consecutive frames. In other words, it defines the positions of the particles in frame $i$, then taking into account the empirically defined behaviour of MTs (e.g. the growth directionality is close to linear with minimal angular changes) and a user-defined search radius, it predicts the position of the particle in the frame $i+1$. If there is a particle in the predicted position, then they will be linked. Similarly is done between frames $i$ and $i-1$. As a result a sub-track is created using at least three consecutive frames. It should be noted, that defining smaller search radius decreases the chances of detecting "false" non-linear sub-tracks, however it also limits the sensitivity of tracking. In any case, if such non-linear sub-tracks are detected, then they are "quality" controlled: they are split in two, and if the resulting sub-tracks are shorter than three frames, then the original sub-track will be discarded.

The candidate sub-tracks are then linked into compound sub-tracks. This step aims to identify and join together the sub-tracks which belong to the same MT. The sub-track linking, similar to particle linking, takes into account the entire population of growth sub-tracks throughout the movie and the linearity of the resulting compound tracks. Also, the time interval between the two selected sub-tracks must be reasonably short in order to prevent cross-linking with another MT (Applegate \& Danuser, 2010).

Track analysis. In this final step of the analysis, the defined sub-tracks are 
A

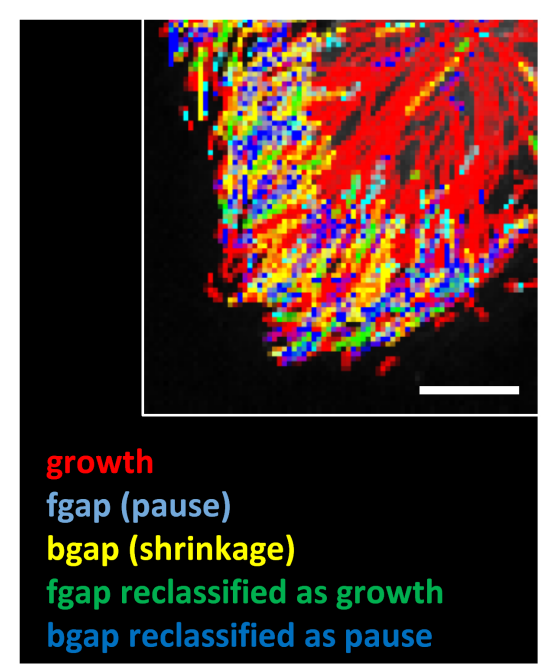

B

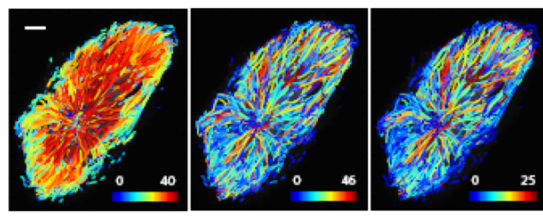

Growth Sub-

tracks

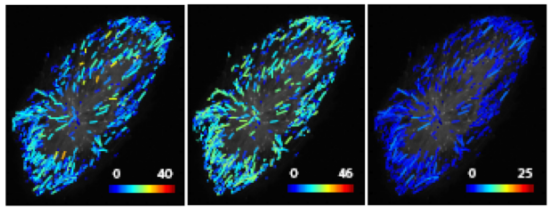

Fgap Sub-tracks (pause events)

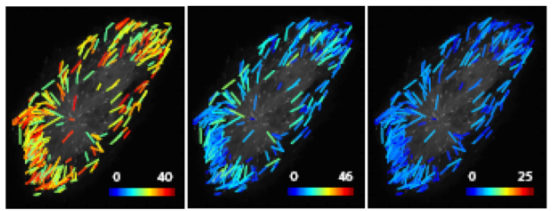

Bgap Sub-tracks

(shrinkage events)

Figure 2.9: Classification of growth sub-tracks. A, An overlay image of track analysis is given showing the classified groups of sub-tracks (in corresponding colours; scale bar, $10 \mu \mathrm{m}$ ). B, Spatial maps of growth, fgap, and bgap sub-tracks colour-coded by speed (left column), lifetime (center column), and displacement (right column) are shown (scale bar, $10 \mu \mathrm{m}$ ). Adapted with modifications from (Applegate $\&$ Danuser, 2010).

classified into growth, fgaps (forward gaps), and bgaps (backward gaps) sub-tracks within a compound track (Figure 2.9). As described above, the compound tracks consist of at least two growth sub-tracks. The gaps between these sub-tracks are coded as fgaps or bgaps, depending on their relative spatial orientation. Fgaps show pause events, when the MT growth continues after an interruption. Bgaps are a representation of catastrophe events: switch from growth to shrinkage. The velocity of each compound track is determined by dividing the displacement between consecutive frames throughout the considered frames.

Gaps can arise for reasons other than true pause or shrinkage. Fgaps can arise due to detection failures or comets moving temporarily out of the focal plane. To exclude such false positive events, the gap speed distributions are stratified according to the general behaviour of MT. If the plus-end displacement speed during such fgaps is greater than $70 \%$ of the growth speed right before the comet disappearance, it is a strong indication of fgaps representing a continuation of growth. Therefore, such fgaps are reclassified as growth phases. The remaining fgaps are assumed to be true pause events.

Bgaps are also analysed regarding them being a true shrinkage. False bgaps can arise from an MT polymer or a positional drift. If the speed of MT plus-end displacement during a bgap is less than the 95th percentile of the speed distribution 
of an fgap classified as a true pause event, then such bgap is considered as a pause rather than a shrinkage with a confidence of $95 \%$. Therefore, it will be reclassified as a pause event, and the bgaps which do not fulfil this requirement will be the true shrinkage events.

After the reclassification of the sub-tracks, a list of phenotypic parameters (Table 2.5) is calculated.

Table 2.5: The information generated by tracking the MT dynamics with U-Track 2.2.0 software (Applegate E Danuser, 2010)

\begin{tabular}{|c|c|c|}
\hline Statistics & Notes about calculation & $\begin{array}{l}\text { Field names in projData } \\
\text { structure }\end{array}$ \\
\hline \multicolumn{3}{|l|}{ Growth statistics } \\
\hline Number of growth sub-tracks & $\begin{array}{l}\text { Calculated after discarding those } \\
\text { beginning in the first frame or } \\
\text { ending in the last frame }\end{array}$ & nGrowths \\
\hline $\begin{array}{l}\text { Growth speed median, mean, and } \\
\text { std }\left(\mu m{ }^{*} \mathrm{~min}^{-1}\right)\end{array}$ & & $\begin{array}{l}\text { growth_speed_median, } \\
\text { growth_speed_mean_std }\end{array}$ \\
\hline $\begin{array}{l}\text { Growth lifetime median, mean, } \\
\text { and std }(s)\end{array}$ & & $\begin{array}{l}\text { growth_lifetime_median, } \\
\text { growth_lifetime_mean_std }\end{array}$ \\
\hline $\begin{array}{l}\text { Growth displacement median, } \\
\text { mean, and std }(\mu \mathrm{m})\end{array}$ & & $\begin{array}{l}\text { growth_length_median, } \\
\text { growth_length_mean_std }\end{array}$ \\
\hline $\begin{array}{l}\text { Frame-to-frame displacements } \\
\left(\text { pixels * } \text { frame }^{-1}\right)\end{array}$ & $\begin{array}{l}\text { Distribution of all displacements } \\
\text { between frame pairs for growth } \\
\text { sub-tracks (instantaneous } \\
\text { velocities) }\end{array}$ & frame2frameDispPix \\
\hline $\begin{array}{l}\text { Frame pair-to-frame pair details } \\
\text { (pixels) and standard deviation of } \\
\text { this distribution }\left(\mu m{ }^{*} \text { min }^{-1}\right)\end{array}$ & $\begin{array}{l}\text { Distribution of the differences } \\
\text { between consecutive frame pairs' } \\
\text { instantaneous velocities }\end{array}$ & $\begin{array}{l}\text { pair2pairDiffPix, } \\
\text { pair2pairDiffMicPerMinStd }\end{array}$ \\
\hline \multicolumn{3}{|l|}{ Forward gap (fgap) statistics } \\
\hline Number of fgap sub-tracks & & nFgaps \\
\hline $\begin{array}{l}\text { Fgap speed median, mean, and } \\
\text { std }\left(\mu m * \mathrm{~min}^{-1}\right)\end{array}$ & & $\begin{array}{l}\text { fgap_speed_median, } \\
\text { fgap_speed_mean_std }\end{array}$ \\
\hline $\begin{array}{l}\text { Fgap lifetime median, mean, and } \\
\text { std (s) }\end{array}$ & & $\begin{array}{l}\text { fgap_lifetime_median, } \\
\text { fgap_lifetime_mean_std }\end{array}$ \\
\hline $\begin{array}{l}\text { Fgap displacement median, mean, } \\
\text { and std }(\mu \mathrm{m})\end{array}$ & & $\begin{array}{l}\text { fgap_length_median, } \\
\text { fgap_length_mean_std }\end{array}$ \\
\hline Fgap frequency, time $\left(s^{-1}\right)$ & $\begin{array}{l}1 / \text { mean }(\mathrm{T}), \text { where } \mathrm{T} \text { is the life- } \\
\text { time of the growth sub-track just } \\
\text { prior to the fgap }\end{array}$ & fgap_freq_time \\
\hline Fgap frequency, length $\left(\mu m^{-1}\right)$ & $\begin{array}{l}1 / \text { mean }(\mathrm{L}) \text {, where } \mathrm{L} \text { is the length } \\
\text { of the growth sub-track just prior } \\
\text { to the fgap }\end{array}$ & fgap_freq_length \\
\hline$\%$ time spent in fgap, individual & $\begin{array}{l}\text { Average value for all fgap- } \\
\text { containing tracks }\end{array}$ & avgIndivPercentTimeFgap \\
\hline \multicolumn{3}{|l|}{ Bulk statistics } \\
\hline \multicolumn{3}{|l|}{ Backward gap (bgap) statistics } \\
\hline Number of bgap sub-tracks & $\begin{array}{l}\text { Calculated after discarding those } \\
\text { bgaps linked to a discarded } \\
\text { growth sub-track (see above) }\end{array}$ & nBgaps \\
\hline $\begin{array}{l}\text { Bgap speed median, mean, and } \\
\text { std }\left(\mu m * m^{*}{ }^{-1}\right)\end{array}$ & & $\begin{array}{l}\text { bgap_speed_median, } \\
\text { bgap_speed_mean_std }\end{array}$ \\
\hline $\begin{array}{l}\text { Bgap lifetime median, mean, and } \\
\text { std }(s)\end{array}$ & & $\begin{array}{l}\text { bgap_lifetime_median, } \\
\text { bgap_lifetime_mean_std }\end{array}$ \\
\hline $\begin{array}{l}\text { Bgap displacement median, mean, } \\
\text { and std }(\mu \mathrm{m})\end{array}$ & & $\begin{array}{l}\text { bgap_length_median, } \\
\text { bgap_length_mean_std }\end{array}$ \\
\hline
\end{tabular}




\section{Table 2.5 (continued)}

\begin{tabular}{|c|c|c|}
\hline Bgap frequency, time $\left(s^{-1}\right)$ & $\begin{array}{l}1 / \text { mean }(\mathrm{T}) \text {, where } \mathrm{T} \text { is the life- } \\
\text { time of the growth sub-track just } \\
\text { prior to the bgap }\end{array}$ & bgap_freq_time \\
\hline Bgap frequency, length $\left(\mu m^{-1}\right)$ & $\begin{array}{l}1 / \text { mean }(\mathrm{L}), \text { where } \mathrm{L} \text { is the length } \\
\text { of the growth sub-track just prior } \\
\text { to the bgap }\end{array}$ & bgap_freq_length \\
\hline \% time spent in bgap, individual & $\begin{array}{l}\text { Average value for all bgap- } \\
\text { containing tracks }\end{array}$ & avgIndivPercentTimeBgap \\
\hline Dynamicity $\left(\mu m{ }^{*}\right.$ min $\left.^{-1}\right)$ & $\begin{array}{l}\text { Collective displacement of all } \\
\text { gap-containing tracks over their } \\
\text { collective lifetimes }\end{array}$ & dynamicity \\
\hline$\%$ time spent in growth, collective & $\begin{array}{l}\text { Total time all MTs spend growing } \\
\text { over the total time all tracks exist }\end{array}$ & percentTimeGrowth \\
\hline$\%$ time spent in fgap, col-lective & $\begin{array}{l}\text { Total time all MTs spend in fgap } \\
\text { over the total time all tracks exist }\end{array}$ & percentTimeFgap \\
\hline$\%$ time spent in bgap, col-lective & $\begin{array}{l}\text { Total time all MTs spend in bgap } \\
\text { over the total time all tracks exist }\end{array}$ & percentTimeBgap \\
\hline \multicolumn{3}{|l|}{ Tracking statistics } \\
\hline Total track number & $\begin{array}{l}\text { Includes those beginning in the } \\
\text { first frame and those ending in } \\
\text { the last frame }\end{array}$ & nTracks \\
\hline$\%$ of all gaps which are fgaps & Sum to $100 \%$ & percentGapsForward \\
\hline$\%$ of all gaps which are bgaps & Sum to $100 \%$ & percentGapsBackward \\
\hline $\begin{array}{l}\text { \% growth sub-tracks linked to } \\
\text { fgaps }\end{array}$ & Sum to $100 \%$ & percentGrowthLinkedForward \\
\hline $\begin{array}{l}\text { \% growth sub-tracks linked to } \\
\text { bgaps }\end{array}$ & & percentGrowthLinkedBackward \\
\hline $\begin{array}{l}\text { \% growth sub-tracks not linked to } \\
\text { gaps }\end{array}$ & & percentGrowthTerminal \\
\hline $\begin{array}{l}\text { Mean frame-to-frame displace- } \\
\text { ment / nearest neighbour distance }\end{array}$ & $\begin{array}{l}\text { How far an average comet travels } \\
\text { between frames compared to } \\
\text { how close its nearest neighbor is; } \\
\text { this is a measure of how difficult } \\
\text { the tracking problem is ( }>0.5 \text { is } \\
\text { difficult) }\end{array}$ & meanDisp2medianNNDistRatio \\
\hline \% fgaps reclassified as growth & $\begin{array}{l}\text { Reclassification occurs when fgap } \\
\text { speed is } \geq 70 \% \text { of the growth } \\
\text { speed prior to comet } \\
\text { disappearance }\end{array}$ & percentFgapsReclass \\
\hline$\%$ fgaps reclassified as growth & $\begin{array}{l}\text { Reclassification occurs when fgap } \\
\text { speed is } \geq 70 \% \text { of the growth } \\
\text { speed prior to comet } \\
\text { disappearance }\end{array}$ & percentFgapsReclass \\
\hline \% bgaps reclassified as pause & $\begin{array}{l}\text { Reclassification occurs when bgap } \\
\text { speed is } \leq \text { the } 95 \text { th percentile of } \\
\text { remaining fgap speeds }\end{array}$ & percentBgapsReclass \\
\hline
\end{tabular}

\subsubsection{Intracellular calcium imaging}

\section{Intracellular calcium imaging in hTERT RPE1 cells}

Intracellular calcium was monitored in hTERT RPE1 cells synchronised at the G0 phase by serum deprivation (see "Synchronisation in G0 phase by serum deprivation"). Fluo-4AM (Thermo Fischer Scientific) was used as intracellular calcium reporter in living cells (Gee et al., 2000). The binding to $\mathrm{Ca}^{2+}$ is only accompanied with 
increase in the intensity of the emitted light, no wavelength shift in the emitted light is observed (Minta, Kao, \& Tsien, 1989). Thus, the fluo-dyes are non-ratiometric and allow to detect only relative changes in cytosolic $\left[\mathrm{Ca}^{2+}\right]$.

In brief, the hTERT RPE1 cells were plated onto 4 -well $\mu$-ibidi plates, and transfected with either $S c r$ - or $K C N H 1$-siRNA. The cells were then washed twice with warm growth medium with no FCS, and incubated with phenol red free DMEM medium supplemented with 1x GlutaMAX for $60 \mathrm{~h}$. It should be noted that the number of the cells per well was adjusted to achieve a cell confluency of approximately $80-85 \%$ after the synchronisation. If allowed to reach full confluency, the growth of the cells would be contact inhibited (Bodnar et al., 1998; X. R. Jiang et al., 1999).

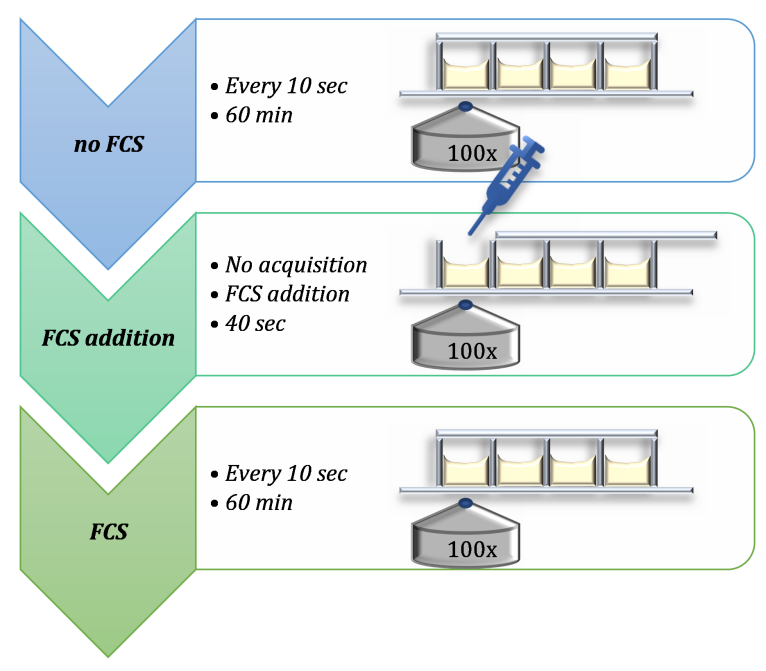

Figure 2.10: A schematic diagram depicts the experimental design of intracellular calcium imaging.

The synchronised cells were incubated with $10 \mu \mathrm{M}$ Fluo-4AM in phenol red free DMEM medium supplemented with GlutaMAX and without FCS. The 4-well plate was placed for image acquisition in an environmental chamber with dark panels (Okolab) at $37^{\circ} \mathrm{C}$ and $5 \%$ $\mathrm{CO}_{2}$. Image acquisition started at 30 min after addition of Fluo4-AM, which was present throughout the entire duration of the experiment.

All images were acquired using a Nikon TiE-Andor inverted microscope (Nikon) as described before. Fluo-4 was excited with the $488 \mathrm{~nm}$ laser line and emission was collected through a 525/30 emission filter. Laser power was set to $15 \%$, and exposure time to $60 \mathrm{~ms}$. In total, 10 positions per well were selected using the multipoint acquisition feature of NIS-Elements software. The timing of the image acquisition is depicted in Figure 2.10. First, the selected positions were imaged in the absence of FCS for 60 min at $10 \mathrm{~s}$ intervals. Afterwards, without removing the plate, $55 \mu \mathrm{l}$ FCS was added to the imaged well to achieve 10\% FCS final concentration. After careful addition of FCS without disturbing the defined positions, imaging was immediately resumed and continued for an additional $60 \mathrm{~min}$. The time interval 
between the last image without FCS and the first image with FCS was fixed to $40 \mathrm{~s}$ throughout all the experiments.

Analysis of relative changes in intracellular $\left[\mathrm{Ca}^{2+}\right]$

The analysis of the obtained intracellular calcium imaging was done in three steps: i) processing of the recorded time-lapse series; ii) background subtraction and calculation of corrected total cell fluorescence (CTCF); iii) analysis of the oscillatory behaviour of the recorded signal.

Processing of time-lapse series. The changes in the intensity of the emitted light were measured using the NIS-Elements software (Nikon). In each recorded image, ten cells were manually selected as regions of interest (ROIs). Only the cells that stayed in the defined ROI throughout the experiment were used for analysis. In addition to sample ROIs, three background ROIs of constant size were identified. These background ROIs were later used for Mean Background Fluorescence calculation. Once the ROIs were defined, using time measurement tool of NIS-Elements software the intensity values were measured for each ROI over time. The data was then exported into an Excel file.

Background subtraction and CTCF calculation. The generated Excel files were reorganised using R-studio open source software (RStudio, Inc). In brief, the script (kindly provided by J. Seidel; see Appendix) first reorganises the file by combining the recorded values for each ROI into following columns, e.g. Time ID, Time (sec), SUM of Intensities 1, Area of ROI 1. Afterwards, CTCF values are calculated using the following formula:

$$
\begin{array}{r}
C T C F=\text { Integrated Density }-(\text { Area of selected cell } \\
* \text { Mean Background Fluorescence })
\end{array}
$$

The CTCFs were calculated for each ROI and time point separately, and the resulting values were exported into a .txt file.

Analysis of the oscillatory behaviour of the recorded signal. The fluctuations in intracellular $\left[\mathrm{Ca}^{2+}\right]$, also known as calcium oscillations, encode information on diverse biological processes. A cell is capable of differentiating these signals by decoding the frequency and the amplitude of the calcium oscillations (Parekh, 2011; 
Samanta \& Parekh, 2017; Smedler \& Uhlén, 2014). Calcium oscillations are reflected in fluctuations in Fluo-4 intensity that can be quantified.

The frequency of the oscillations of the acquired images was defined using an open source MATLAB-based tool "Spectral analysis of calcium oscillations", version 3.0 (Uhlen, 2004). This tool deploys the Fourier transform principle: the time and frequency domains are swapped (Fourier, 1822), and as an output creates a power spectrum. The detailed explanation and the script can be found in Uhlen (2004). In brief, collected and calculated values from each ROI were divided into five temporal segments: i) total duration (-3600 - $3600 \mathrm{sec})$; ii) before FCS addition (-3600 - 00 sec); iii) after FCS addition (00 - $3600 \mathrm{sec})$; iv) the first peak observed immediately upon FCS addition (00 - $60 \mathrm{sec})$; and v) after FCS addition excluding the first peak $(60-3600 \mathrm{sec})$. The power spectrum for each segment was generated and the mean frequency value defined.

In addition to frequency, prominence and FWHM (full width at half maximum) were calculated for the above-listed segments in the frequency domain, and the total

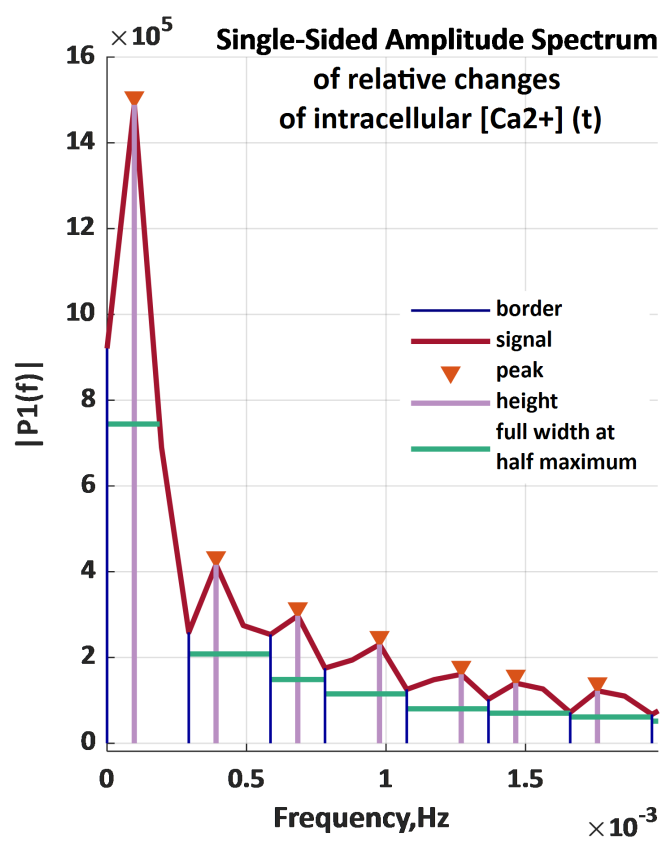

Figure 2.11: Identification of peaks in the single-sided amplitude spectrum of intracellular $\left[\mathrm{Ca}^{2+}\right]$. number of peaks and total AUC (area under the curve) - in the time domain. The prominence shows how much a given peak stands out of its neighbour peaks due to its intrinsic height and location relative to other peaks. Thus a low isolated peak can be more prominent than one that is higher but is an otherwise unremarkable member of a tall range (The MathWorks, 2018). A new MATLABbased script similarly to the above mentioned one and using Fourier transform was developed. In brief, this second script first fits a linear function to the "Before FCS" segment in order to compensate for the baseline drift in the collected data. This drift results from a combination of on one hand photobleaching, and, on the other, continuous loading of the 
cells with Fluo-4AM. The resulting line is extrapolated to the entire duration of the experiment and then subtracted from the measured values, resulting in a normalised CTCFs.

The peaks in the CTCF spectrum are identified and indexed with their time values using the "findpeaks" function in MATLAB. This function identifies local maxima (peaks) of the input signal vector by comparing value $i$ in the given spectrum with value $i-1$ and value $i+1$. If the value $i$ is larger than the two neighbours than it will be considered as a peak. In addition to the peak values, the function also returns a vector with the locations of the peaks in the time or frequency domain, the prominence and the FWHM (Figure 2.11). The AUC values in the CTCF spectrum are also defined.

The second part of the script repeats the aforementioned "Spectral analysis of calcium oscillations", but this newly developed script identifies number, prominence and location (in relation to the frequency domain) of peaks, and also FWHM. The new script, given in the Appendix, is simplified due to its length and depicts the analysis of only one cell and segment.

\subsection{Statistical analysis}

All data were statistically analysed using GraphPad Prism software v8 (GraphPad Software, Inc.). Two-tailed Student's t-test was used for the experiments with only two conditions. The MT dynamics and comet assay experiments with three groups and one variables were analysed using one-way ANOVA (analysis of variance). For the analysis of the synchronisation and immunoblotting experiments, two-way ANOVA with Bonferroni-adjusted post hoc tests were used. All data are shown as mean standard deviation (SD) or standard error of the mean $(\mathrm{SEM})$, where $\mathrm{P}$ value $<0.05$ was considered statistically significant. 
Materials \& Methods 


\section{Chapter 3}

\section{Results}

We have previously shown that Kv10.1 expression is temporally restricted to a short time window during the G2 and M phases of cell cycle, and that downregulation of Kv10.1 induces accumulation of cells in that phase (Urrego et al., 2016). Therefore, we speculated that Kv10.1 plays a role in the regulation of processes happening in the $\mathrm{G} 2 / \mathrm{M}$ phase.

As already discussed in the "Introduction", two major checkpoints function in G2 and M phases of cell cycle: the DNA damage-induced checkpoint and the spindle assembly checkpoint (SAC), respectively (Lodish, 2000). Activation of either of these checkpoints, which share some components, will lead to an arrest in cell cycle progression. I therefore first characterised the status of both of the checkpoints using two models: mammalian normal and cancer cells.

As a human cancer cell model system, we continued to use HeLa cells as in our previous work. The line is one of the most widely used for cell cycle research (Bowey-Dellinger et al., 2017; Masters, 2002), because HeLa cells can efficiently be synchronised at different phases of cell cycle (Ma \& Poon, 2011); HeLa cells have high expression of Kv10.1 (Pardo et al., 1999) without completely losing the cell cycle-dependent expression pattern (Urrego et al., 2016).

As a non-cancer cell line, hTERT RPE1 cells were used. Likewise, hTERT RPE1 cells express Kv10.1 at G2/M phase (A. Sánchez, Urrego, \& Pardo, 2016b). Despite being immortalised with the introduction of the TRT oncogene (human telomerase reverse transcriptase), these cells retain a normal, non-tumour phenotype: they display contact inhibition of growth (X. R. Jiang et al., 1999) and primary cilia when deprived of growth factors (Plotnikova, Pugacheva, \& Golemis, 2009). 


\subsection{Kv10.1 and DNA damage-induced checkpoint}

\subsubsection{Downregulation of Kv10.1 partially activates DNA damage-induced checkpoint}

As stated previously arrest in G2 can be due to the activation of the DNA damageinduced checkpoint, which in mammalian cells consists mainly of the ATM(ATR)/ CHK2(CHK1)-p53/MDM2-p21 pathway (Nyberg et al., 2002). We therefore investigated the activity of the DNA damage-induced checkpoint upon Kv10.1 downregulation. HeLa cells were synchronised using a double thymidine block protocol and samples were collected as described above. The abundance of DDR proteins was subsequently determined by immunoblotting. Analysis of the images revealed that some of the DDR components are elevated in Kv10.1-deficient HeLa cells (Figure 3.1).

The total level of CHK2 was clearly increased in Kv10.1-deficient HeLa cells. The increase reached approximately $70 \%$ at $0-8 \mathrm{~h}$ after block release (expression normalised to actin: $0.24 \pm 0.01$ vs $0.14 \pm 0.01$ in the control). Kv10.1 downregulation conferred cell-cycle dependence to CHK2 levels, which were stable in the control over time, but increased to a peak at $8 \mathrm{~h}$ after release from thymidine block and decayed thereafter in KD cells to the levels of the control cells. Interestingly, the active phosphorylated forms of CHK1 (Ser345, a phosphorylation site for ATR; K. Jiang et al., 2003; Zhao \& Piwnica-Worms, 2001) and ATR (Ser428, an activating phosphorylation site; J. Smith, Mun Tho, Xu, \& A. Gillespie, 2010; Vauzour, Vafeiadou, Rice-Evans, Cadenas, \& Spencer, 2007) were also upregulated in Kv10.1 KD cells. The total level of p-CHK1 was increased by $60 \%$ relative to the control group $(0.09 \pm 0.02$ vs $0.14 \pm 0.03)$, whereas the total amount of p-ATR in KD cells doubled that of the control $(0.41 \pm 0.03$ vs $0.20 \pm 0.01$, respectively). However, no significant differences in the total amount of p-BRCA1 (breast cancer 1, early onset; Ser1524, a phosphorylation site for ATR; S.-Y. Lin, Li, Stewart, \& Elledge, 2004), CHK1, p21 and phospho-histone H2A.X ( $\gamma$ H2A.X; Ser139, phosphorylated by ATM; Burma, Chen, Murphy, Kurimasa, \& Chen, 2001) or ATR (DeLoughery, Luczak, Ortega-Atienza, \& Zhitkovich, 2015) were observed. 
A

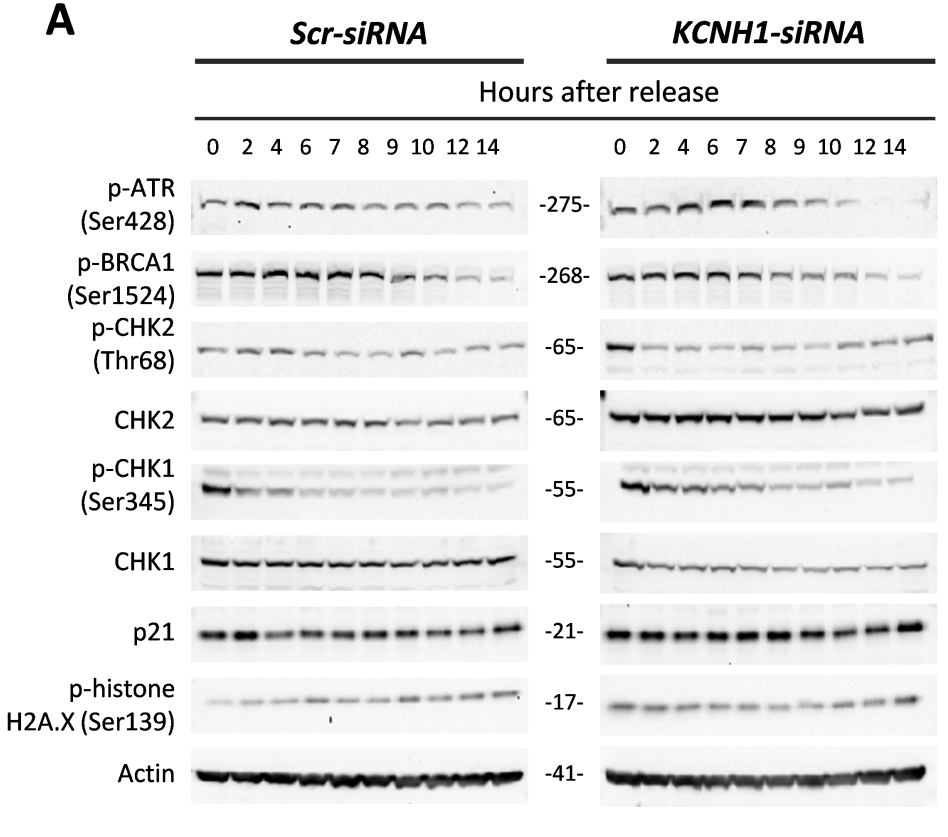

B

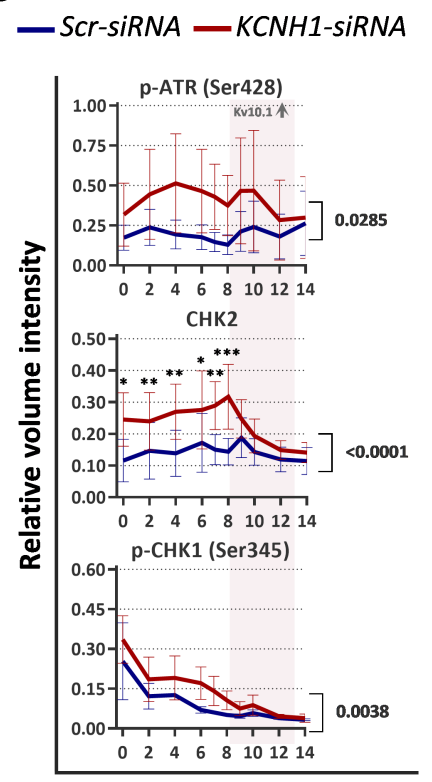

Hours after release

Figure 3.1: Kv10.1 downregulation induces accumulation of some proteins involved in DNA damage-induced checkpoint in HeLa cells. Hela cells were transiently transfected with either Scr- or KCNH1-siRNA, synchronised with double thymidine block at the G1/S border, and subsequently released from the block. The samples were collected every $2 h$ between $0-6 h$ and $10-14 h$, and every $1 h$ during $6-10 h$ after the release from the block; the abundance of proteins in the samples was analysed by immunoblot. A, Representative images of immunoblots. $B$, Densitometry analysis of all immunoblot repeats. The time interval corresponding to Kv10.1 expression is indicated in pink ( $n=3$ of independent synchronisation experiments, mean \pm SEM is shown; two-way ANOVA, repeated measures, the given $P$ value shows the significance of total difference between the two groups, and "*" - between each time point, ${ }^{*} P=0.05$ ).

Taken together, these results suggest a possible activation of DDR; in particular, the upstream part of the pathway appears activated in KD cells.

\subsubsection{The activation of DDR in Kv10.1-deficient cells does not obey actual DNA damage}

The activation of the DNA damage-induced checkpoint can happen both in the presence (Shaltiel et al., 2015) or absence of DNA damage (Baumann, 2014; Burgess, Burman, Kruhlak, \& Misteli, 2014; Cipressa \& Cenci, 2013; Eliezer, Argaman, Kornowski, Roniger, \& Goldberg, 2014; Kidiyoor, Kumar, \& Foiani, 2016; Lawrence, Chau, \& Engebrecht, 2015). I, therefore, studied the presence and extent of DNA lesions to identify the conditions for DDR activation. To this end, I used one of the methods that detects DNA lesions at low levels, single-cell gel electrophoresis (also known as comet assay). In particular, the alkaline variation of the method detects 
A

H2O2 treated

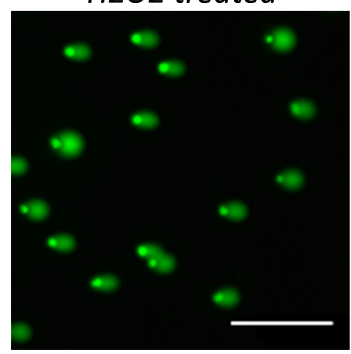

Neg Control
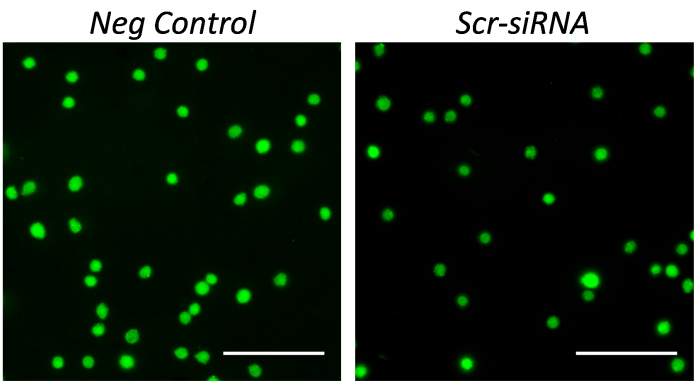

KCNH1-SiRNA

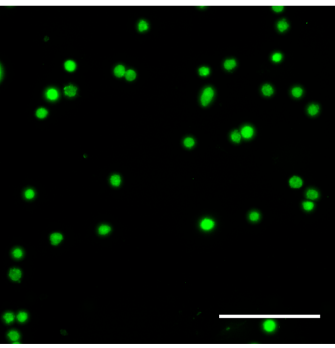

B

HeLa cells

C
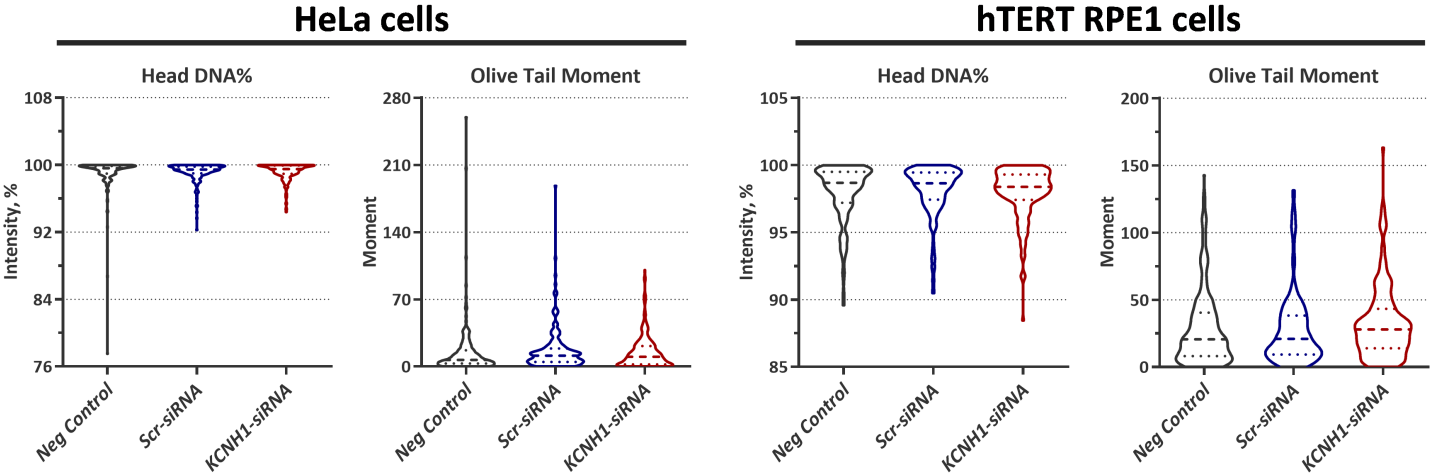

Figure 3.2: Partial activation of DNA damage-induced checkpoint in HeLa cells upon Kv10.1 downregulation is not due to increase in DNA damage. Hela cells were transiently transfected with either Scr- or KCNH1-siRNA, allowed to reach approximately 70\% confluency, harvested and immediately used for single-cell gel electrophoresis (comet assay). A, The DNA comets were stained with SYBR-Gold and visualised with a fluorescence microscope. Representative images are given (max intensity projection, z-stepsize $0.2 \mu \mathrm{m}$, scale bar, $200 \mu \mathrm{m}$ ). $B-C$, The comets were analysed with CASPLab software and Head DNA\% and Olive Tail Moment are plotted in $B$ for HeLa cells and in $C$ for hTERT RPE1 cells $(n=5$ independent experiments with $>750$ comets per condition; one-way ANOVA, median and 25 and 75 percentiles are shown, $\left.{ }^{*} P=0.05\right)$.

SSBs, DSBs and alkaline labile sites (Singh et al., 1988; Speit \& Rothfuss, 2012).

The alkaline comet assay in asynchronously growing HeLa and hTERT RPE1 cells did not reveal any significant differences in DNA damage levels between control and Kv10.1 deficient cells (Figure 3.2) after analysis of DNA comets with CASPLab software (Końca et al., 2003). Therefore, the changes in protein levels upon Kv10.1 depletion are not caused by DNA damage. 


\subsection{Kv10.1 and Spindle Assembly Checkpoint}

\subsubsection{SAC is activated in Kv10.1-deficient HeLa cells}

The spindle assembly checkpoint is a surveillance mechanism that delays anaphase onset until all chromosomes are correctly attached in a bipolar fashion to the mitotic spindle (May \& Hardwick, 2006). An increasing body of evidence indicates that DDR proteins also have a mitotic role in regulating the spindle assembly checkpoint (SAC; Eliezer et al., 2014; Lawrence et al., 2015). Therefore, the accumulation of DDR proteins that we observe could reflect activation of SAC.

To test this, HeLa cells were transfected with either Scr- or KCNH1-siRNA and synchronised at the G1/S border using double thymidine block. Samples of synchronised cells were collected every $2 \mathrm{~h}$ and analysed by WB (Figure 3.3, A) . Densitometry analysis of the immunoblots showed a statistically significant increase in total levels of MAD2L1 and CDC20 in Kv10.1 KD cells (Figure 3.3, B). MAD2L1 total protein levels were $27 \%$ higher in Kv10.1-deficient HeLa cells $(0.26 \pm 0.02$ compared to $0.19 \pm 0.02$ in control cells). Only a moderate 20\% increase in CDC20 was observed in Kv10.1 KD cells $(0.28 \pm 0.05$ vs $0.23 \pm 0.04)$. No differences were observed in BUB3 levels, another component of the SAC; nevertheless, SAC appears to be at least partially more active in $\mathrm{KD}$ cells.

Aurora B, one of the critical regulators of cell cycle, was downregulated by $35 \%$ in Kv10.1-deficient HeLa cells $(0.29 \pm 0.03$ vs $0.40 \pm 0.06$ in the control cells $)$. It is important to note that Aurora B needs to be relocated at the kinetochores to exert its function; such localisation of Aurora B requires MAD2 (Shandilya, Medler, \& Roberts, 2016), and CHK1-mediated phosphorylation of Aurora B increases its activity at centromeres (Zachos et al., 2007). As already demonstrated above, Kv10.1 KD induces an increase in both p-CHK1 (Figure 3.1) and MAD2L1 (Figure 3.3). Therefore, although Aurora B is overall less abundant, the conditions for more efficient activation of Aurora B at the kinetochores are present in Kv10.1 KD cells.

pVHL, the von Hippel-Lindau protein, is also involved in SAC. pVHL is a tumour suppressor protein, and its mutation leads to von Hippel-Lindau syndrome, a condition characterised by benign tumours in different locations with a tendency to malignisation (Varshney et al., 2017). The role of pVHL in response to hypoxia 

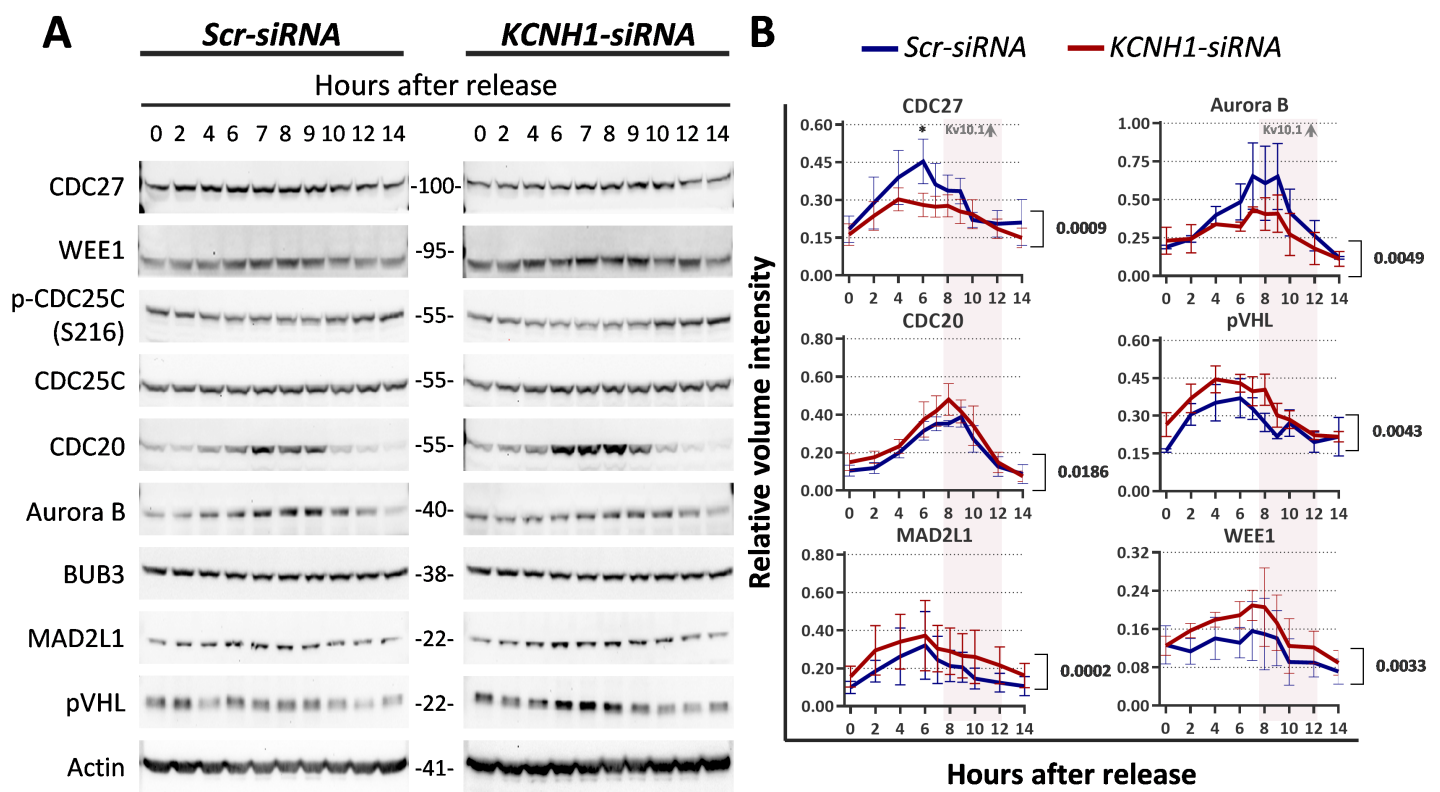

Figure 3.3: Kv10.1 deficiency leads to accumulation of SAC proteins in mitosis in HeLa cells. Hela cells were transiently transfected with either Scr-or KCNH1-siRNA, and synchronised with double thymidine block at G1/S border, and subsequently released from the block. The samples were collected every $2 h$ between $0-6 h$ and $10-14 h$, and every $1 h$ during $6-10$ $h$ and applied to immunoblot analysis. A, Representative images of immunoblots are shown. B, Densitometry analysis of all immunoblot repeats are given with the time interval corresponding to Kv10.1 expression highlighted in pink $(n=3$ independent synchronisation experiments; two-way ANOVA, repeated measures, mean $\pm S E M$ is shown, the given $P$ value shows the significance of total difference between the two groups, and "*" - between each time point, ${ }^{*} P=0.05$ ).

and HIF $1 \alpha$ homeostasis (Q. Zhang \& Yang, 2012) is well established. pVHL has been also shown to reduce chromosome instability via increase of MAD2 levels (Hell, Duda, Weber, Moch, \& Krek, 2014; Thoma et al., 2009), which in turn enhances SAC response (Subramanian \& Kapoor, 2013). Immunoblot analysis showed a moderate, but statistically significant increase in total levels of pVHL in Kv10.1 KD cells (0.34 \pm 0.03 vs $0.27 \pm 0.02$ in control Figure 3.3, A). Therefore, our results in combination with those reported in the literature allow us to speculate that the SAC response in Kv10.1 knockdown cells is possibly stronger.

Interestingly, the APC/C protein CDC27 was downregulated in Kv10.1 KD cells $(0.24 \pm 0.02)$ by $20 \%$ with respect to the control group $(0.30 \pm 0.03)$. Notably, the most significant difference between the groups was observed at $6 \mathrm{~h}$ after the release (by 60\%: $0.45 \pm 0.09$ in control vs $0.28 \pm 0.05$ in KD cells). This observation may point out that Kv10.1-deficient cells transition from metaphase-to-anaphase at later time points than the control cells. In this scenario, a shift in the peak of CDC27 towards later time points in Kv10.1 KD cells should be observed. However, 
the timepoint corresponding to the peak of CDC27 is the same as in both groups (Figure 3.3, B).

The lack of CDC27, which is required for APC/C activity (King et al., 1995), could be compensated by upregulation of other factors. A plausible candidate is WEE1, a kinase responsible for CDK1-Cyclin B1 complex inactivation both at the entry into mitosis (Schmidt et al., 2017) and at the metaphase-to-anaphase transition (Chow, Poon, \& Ma, 2011). Overexpression of Swe1 (the paralog of WEE1 in budding yeast), results in the arrest of cells with reduced APC activity in metaphase in vitro and in vivo (Lianga et al., 2013). When we studied the profile of WEE1 expression during cell cycle progression on the protein level, we observed an upregulation in total WEE1 protein in Kv10.1 deficient cells $(0.16 \pm 0.01)$ by $30 \%$ in comparison to the control group $(0.12 \pm 0.01$, Figure 3.3, B). This upregulation of WEE1 may compensate for by taking over the inhibition of cyclin B1/CDK1 complex and thus trigger the metaphase-to-anaphase transition (reviewed in Schmidt et al., 2017; Visconti, Palazzo, Della Monica, \& Grieco, 2012). Alternatively, WEE1 may prevent the premature activation of $\mathrm{APC} / \mathrm{C}$ and consequently the formation of lagging chromosomes. However, slightly broader peaks of CDC20 and WEE1 at G2/M phase ( $7-12 \mathrm{~h}$ after thymidine block), as well as our previous observations (Urrego et al., 2016) speak against the former possibility and hint to extended and stronger SAC activity and prevention of premature activation of APC/C.

\subsubsection{Activation of SAC in Kv10.1-deficient cells prevents the formation of lagging chromosomes during anaphase onset}

SAC is a surveillance mechanism that delays anaphase onset until all chromosomes are correctly attached in a bipolar fashion to the mitotic spindle (May \& Hardwick, 2006). We observed an upregulation of major components of SAC in synchronised HeLa cells depleted of Kv10.1, an observation that raises two questions. i): Is the upregulation of SAC components due to a delay in the chromosomal alignment? And ii): Is the stronger and longer SAC in Kv10.1 KD cells more efficient in decreasing the occurrence of lagging chromosomes? 
A
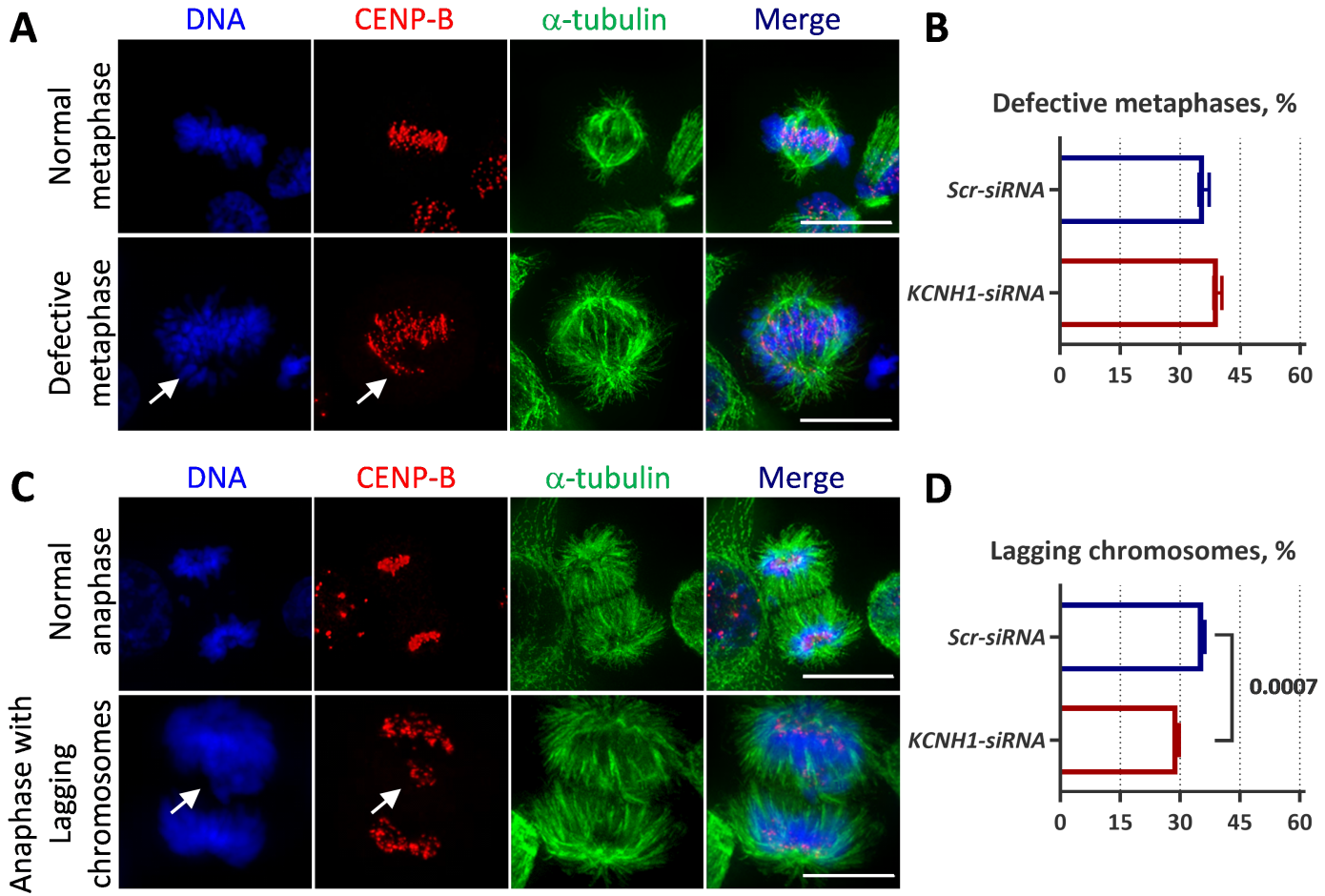

Figure 3.4: Kv10.1 depletion in HeLa cells does not affect the chromosomal alignment at metaphase and decreases the occurrence of lagging chromosomes. HeLa cells were transiently transfected with the indicated siRNAs and synchronised at prophase with double thymidine and nocodazole treatments. Afterwards, nocodazole was removed and the cells were released into either fresh medium for 50 min (anaphase) or fresh medium containing $15 \mu M$ MG132 for 2 $h$ (metaphase). The cells were fixed with $2 \%$ pfa in PHEM (see "Immunofluorescence staining") and permeabilised with ice-cold methanol. The mitotic spindle was visualised by $\alpha$-tubulin staining (green), each chromosome kinetochore through CENP-B staining (red), and DNA was stained using Hoechst 33342 (blue; scale bar, $10 \mu \mathrm{m}$ ). A, A metaphase is considered defective when chromosomes (overlap of CENP-B and Hoechst stains) are not properly aligned (indicated with arrows) or damaged DNA (only Hoechst positive) is present. Example images of normal and defective metaphases are shown. The percentage of defective metaphases from the total number of metaphases was calculated and plotted in $B$ (600 metaphases per experiment, $n=4$ independent synchronisation experiments; unpaired two-tailed Student's t-test, mean \pm SEM is shown). C, Lagging chromosomes in anaphase are detected as CENP-B-and Hoechst-positive signal located at the middle part rather than at the vertices of the spindle (arrows). D, The graph shows the percentage of anaphases with lagging chromosomes from the total number of anaphases (400 anaphases per experiment, $n=4$ independent synchronisation experiments; unpaired two-tailed Student's t-test, mean \pm SEM is shown).

We approached these questions using again HeLa cells transfected with either Scr- or KCNH1-siRNA and synchronised at metaphase, to assess the alignment of chromosomes, and at anaphase, to assess the occurrence of lagging chromosomes. The synchronised cells were fixed and the bipolar mitotic spindle, kinetochores and DNA were visualised on a confocal fluorescence microscope (Figure 3.4, A). Quantification of so-called "defective metaphases", i.e., metaphases with misaligned chromosomes or with damaged DNA (negative for CENP-B, a kinetochore marker), showed no differences between the studied groups (Figure 3.4, B). 
Nevertheless, the question of whether the stronger and longer SAC affects the occurrence of lagging chromosomes still remains. As expected, HeLa cells transfected with $K C N H 1$-siRNA had approximately 20\% less lagging chromosomes $(29.32 \pm$ $0.45 \%$ vs $35.78 \pm 0.45 \%$, Figure 3.4 , D). Therefore, the detected increase in SAC in Kv10.1-deficient HeLa cells facilitates proper chromosome segregation during anaphase.

\subsubsection{Kv10.1 knockdown results in higher PLK1 activity}

During mitosis, sister chromatids must become bioriented on the mitotic spindle, i.e., one chromatid is attached by its kinetochore region to MTs growing from one spindle pole, and its sister chromatid to the MTs of the opposite pole. Once this configuration is achieved for every single chromatid pair, SAC will be inactivated and allow the cell to transition from metaphase-to-anaphase. Since kinetochore-MT interactions form stochastically, the whole process must be supervised and regulated to ensure that improper attachments are continually removed in a process termed error-correction (Saurin, 2018). Thus, an alteration in kinetochore-MT attachments in HeLa cells deficient of Kv10.1 can explain the accumulation of SAC proteins and the decrease in the number of lagging chromosomes.

Recently, a novel pathway involving the Aurora B-PLK1-MCAK axis has been suggested to regulate the accuracy of kinetochore-MT attachments (Shao et al., 2015). In the previous paragraphs, we speculated the possibility of more efficient activation of Aurora B at the kinetochores despite lower amounts of total Aurora B in Kv10.1 KD cells (Figure 3.3). To further assess the involvement of the Aurora B-PLK1-MCAK pathway, we tested the changes in PLK1 over time in synchronised HeLa cells transiently transfected with either a $S c r$ - or KCNH1-siRNA (Figure 3.5, A and B). Total PLK1 level was 30\% lower in cells lacking the channel (0.11 \pm $0.01)$ than in the control cells $(0.16 \pm 0.02)$ based on the densitometry analysis of the immunoblots. However, this did not correlate with the increased PLK1 activity. When measuring the enzymatic activity of PLK1 in HeLa cell lysates collected at the G1/S border (double thymidine protocol) and prophase (thymidine-nocodazole protocol), the cells in prophase with downregulated Kv10.1 had moderately higher PLK1 activity (Figure 3.5, C). Similarly, higher activity of PLK1 was observed in 
A
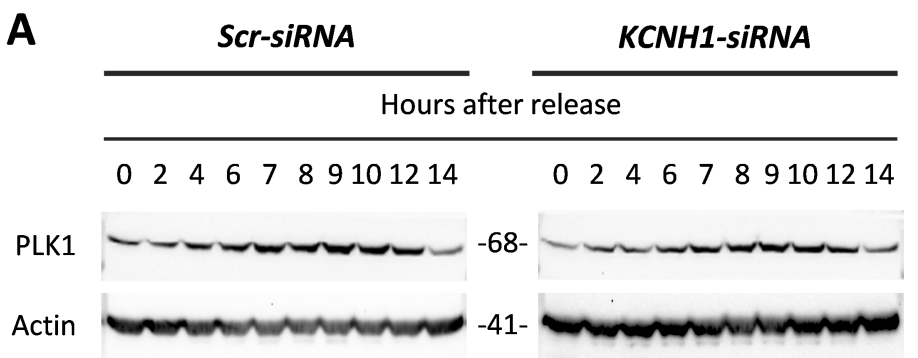

C

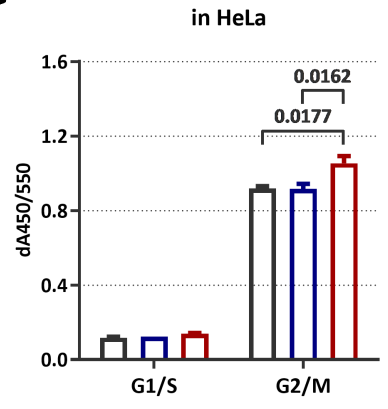

D

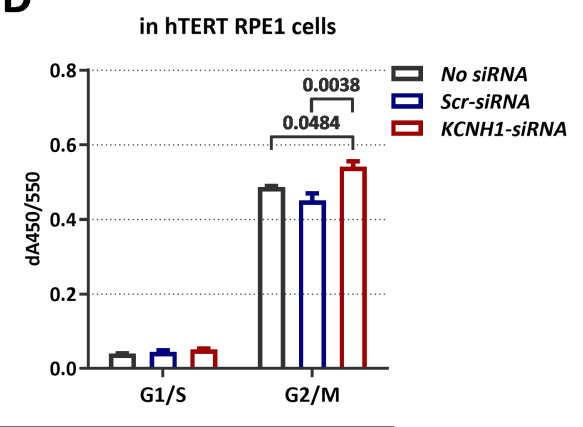

B

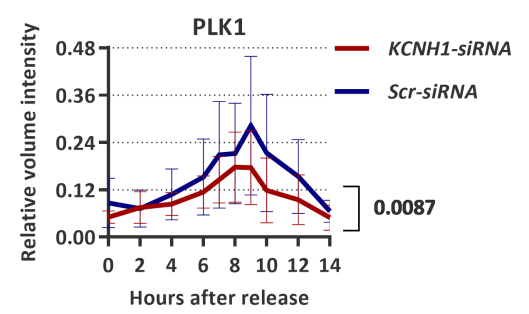

PLK1 activity

Figure 3.5: PLK1 enzymatic activity is higher in Kv10.1 depleted cells despite lower abundance of total PLK1 protein. A-B, HeLa cells were transiently transfected with the respective siRNA and synchronised at the G1/S border via double thymidine block. Synchronised cell aliquots were collected every $2 h$ between $0-6 h$ and $10-14 h$, and every $1 h$ during $6-10 h$ after the release from the block; isolated protein samples were subjected to immunoblot analysis. A, A representative image of such immunoblots is given (50 $\mu \mathrm{g}$ protein per lane, actin served as a reference protein). B, Densitometry analysis of immunoblot images ( $n=3$ independent synchronisation experiments; two-way ANOVA, repeated measurements, mean \pm SEM is shown). C, HeLa cells were synchronised at G1/S with double thymidine block and G2/M with thymidine-nocodazole block. Samples were collected at the corresponding phases of the cell cycle and PLK1 enzymatic activity was measured using an ELISA-based approach. The absorbance of the reaction mixture was measured and plotted ( $n=2$ independent synchronisation experiments; two-way ANOVA, repeated measurements, mean \pm SEM is shown).

Kv10.1-depleted hTERT RPE1 cells synchronised in prophase (Figure 3.5, D).

Thus, despite the decreased level of total protein, the enzymatic activity of PLK1 is higher in Kv10.1 deficient cells. Therefore, we can speculate that, indeed, SAC stays active over more extended period in the KD cells, resulting in a more efficient error-correction. It should nevertheless be noted that this observation should be treated carefully, as the disruption of the dynamic balance of activities of any of the enzymes involved in the regulation of either SAC or error-correction may have opposing effects. 
A

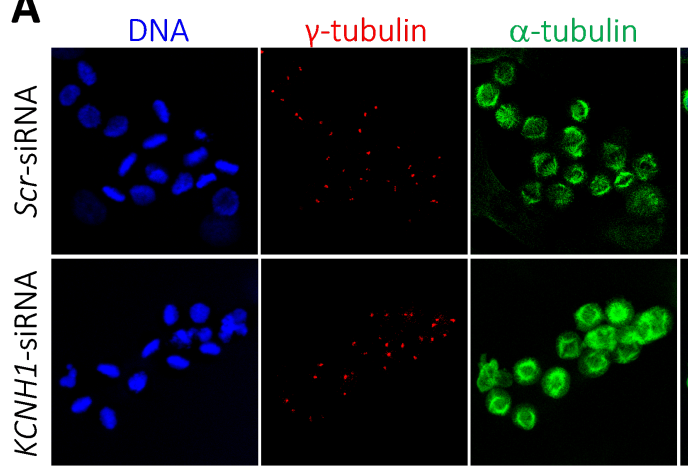

B

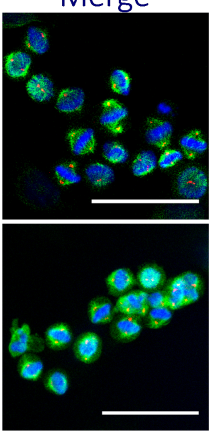

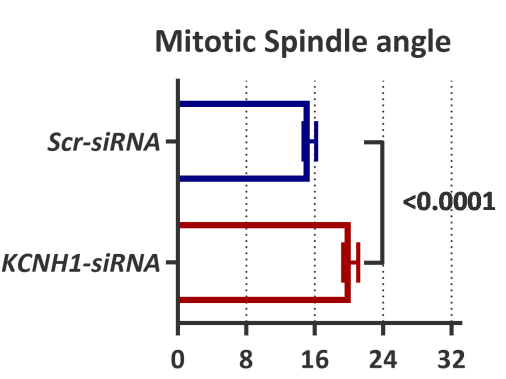

Figure 3.6: An altered mitotic spindle angle is observed in Kv10.1-depleted HeLa cells. HeLa cells were transfected with siRNA and synchronised in metaphase by consecutive treatment with thymidine, nocodazole and MG132. A, Maxima projections of z-stacks (0.2 $\mu \mathrm{m}$ stepsize) of mitotic cells in metaphase (scale bar, $50 \mu \mathrm{m}$ ), where $B$, the spindle angle was determined (>50 events per experiment, $n=5$ independent synchronisation experiments; Student's t-test, twotailed, mean \pm SEM are shown).

\subsubsection{Altered mitotic spindle angle is observed upon loss} of $\mathrm{Kv10.1}$

PLK1 has been described to regulate mitotic spindle orientation by modulating the nucleation and growth of astral MTs from the centrosomes (Hanafusa et al., 2015; Miyamoto et al., 2017; Sana, Keshri, Rajeevan, Kapoor, \& Kotak, 2018). Moreover, pVHL (also increased in Kv10.1 KD cells) has been described to regulate the proper orientation of the mitotic spindle by stabilisation of astral MTs (Hell et al., 2014; Hergovich, Lisztwan, Barry, Ballschmieter, \& Krek, 2002; Lolkema et al., 2007; Thoma et al., 2009). Interestingly, single HeLa cells with greater tilting of the mitotic spindle required more time for biorientation of sister kinetochores due to prolonged activation of the spindle checkpoint (Charnley, Anderegg, Holtackers, Textor, \& Meraldi, 2013). Hence, we checked whether mitotic spindle orientation might also be altered in the absence of Kv10.1.

Similarly to the assessment of defective metaphases, control and with transient downregulation of Kv10.1 HeLa cells were synchronised at metaphase, fixed and the mitotic spindle was visualised on a confocal microscope (Figure 3.6, A). As expected, the mitotic spindle angle was greater in the Kv10.1 KD HeLa cells than in the control cells with mean angle values of $20.14 \pm 0.89$ and $15.41 \pm 0.74$ degrees, respectively (Figure 3.6, B).

Hence, we conclude that the changes in the mitotic spindle angle may be the 
cause for the observed increase in PLK1 and pVHL activities, and subseqently extended SAC - a compensatory mechanism deployed by the cell to rescue the correct positioning of the mitotic spindle by influencing the astral MT dynamics. Hence, experiments aiming to observe the behaviour of MT dynamics globally should be performed.

\subsection{Kv10.1 and microtubule dynamics}

\subsubsection{Downregulation of Kv10.1 increases the occurrence of asymmetric monopolar mitotic spindles in HeLa cells}

In previous paragraphs we showed that the cells with less Kv10.1 have stronger and longer SAC, a bigger spindle angle and less lagging chromosomes during anaphase. Both SAC and spindle orientation, as mentioned above, are highly dependent on MT dynamics and can influence or be influenced by them (Kotak, 2019; Saurin, 2018; H.-Y. Wu et al., 2017). Therefore, we questioned whether the described phenotypic and biochemical changes are due to alterations in MT dynamics when Kv10.1 is lost. A pilot experiment using DME-assay (Ertych et al., 2014) was performed to answer this question.

DME is a small molecule that inhibits the kinesin Eg5 motor protein resulting in a formation of monopolar spindles (Mayer et al., 1999; Müller et al., 2006). Interestingly, cells with altered MT dynamics form asymmetric monopolar spindles. It should be noted that the treatment with DME itself does not affect the assembly rates of MT plus-tips (Ertych et al., 2014). HeLa cells were treated with $2.5 \mu \mathrm{M}$ DME and the mitotic spindles were visually checked under a fluorescence microscope (Figure 3.7, A). The monopolar mitotic spindles were manually counted and the percentage of asymmetric events out of all the monopolar spindles was calculated. As shown in Figure 3.7, B, the percentage of asymmetric monopolar spindles was higher in Kv10.1 KD HeLa cells than in the control cells. Thus, this result already indicates alterations in MT dynamics in Kv10.1 deficient cells, which, however, should be further validated by directly measuring MT dynamics in live cells. 


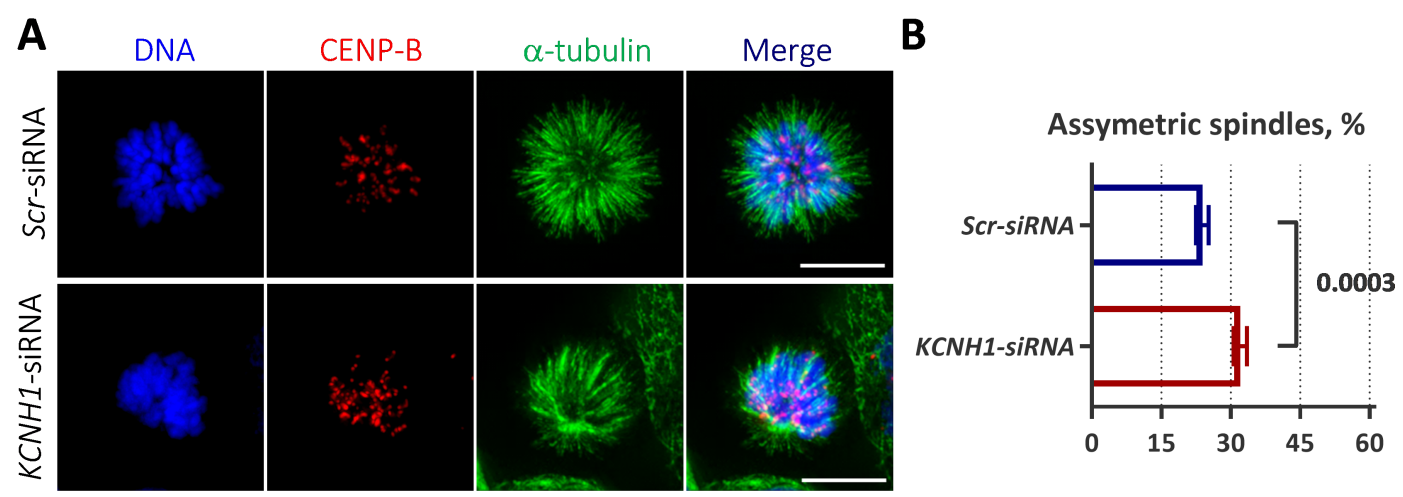

Figure 3.7: Increased MT plus end growth rate is observed upon Kv10.1 downregulation in Hela cells. HeLa cells were transiently transfected with a respective siRNA, grown until $70 \%$ confluency and treated with $2.5 \mu M$ DME for 4 h, enriching the cells in monopolar mitotic spindles. The cells were fixed with $2 \%$ pfa in PHEM, permeabilised with ice-cold methanol. Mitotic spindle were visualised via $\alpha$-tubulin staining (green), each chromosome kinetochore - CENP-B (red), and DNA - Hoechst 33342 (blue; scale bar, $10 \mu \mathrm{m}$ ). A, Representative images of monopolar spindles are shown. B, The percentage of asymmetric monopolar spindles from total number of monopolar spindles was calculated and plotted (1500 monopolar spindle per condition per experiment, $n=3$ independent synchronisation experiments; unpaired two-tailed Student's t-test, mean \pm SEM is shown).

\subsubsection{The absence of Kv10.1 results in an increased MT plus-end growth}

To confirm the preliminary data obtained using DME-assay, we measured the MT plus-end growth rates directly in living cells expressing EB3-tdTomato. The cells were transiently transfected with either Scr-or KCNH1-siRNA, and growing MTs were monitored. MT plus-end growth speed, length and lifetime were measured with U-Track software (Figure 3.8, A, supplementary video 1 - 4).

Strikingly, all measured parameters differed between the control and KD cells both in HeLa (Figure 3.8, B) and hTERT RPE1 (Figure 3.8, C). The growth speed was increased both in Kv10.1 KD HeLa (18.80 $\pm 0.22 \mu \mathrm{m} * \mathrm{~min}^{-1}$ compared to 17.68 \pm 0.24 in control cells $)$ and KD hTERT RPE1 $\left(19 \pm 0.47 \mu \mathrm{m} * \mathrm{~min}^{-1}\right.$ vs $14.86 \pm$ 0.39 in control) cells by $1 \mu \mathrm{m} * \mathrm{~min}^{-1}$ and $4.2 \mu \mathrm{m} * \mathrm{~min}^{-1}$, respectively. Similarly, the growth length, which is the displacement of a growing tip during the recording time $(1 \mathrm{~min})$, was increased by $0.02 \mu \mathrm{m}$ in Kv10.1 KD HeLa $(0.58 \pm 0.01)$, and almost by $0.08 \mu \mathrm{m}$ in KD hTERT RPE1 cells $(0.58 \pm 0.01)$ relative to their controls: $0.56 \pm 0.01 \mu \mathrm{m}$ and $0.50 \pm 0.01 \mu \mathrm{m}$, respectively. The increase in growth speed and length was accompanied by lesser growth lifetime in both cell lines upon Kv10.1 loss. In HeLa cells, the difference in growth lifetime between the two groups was not 


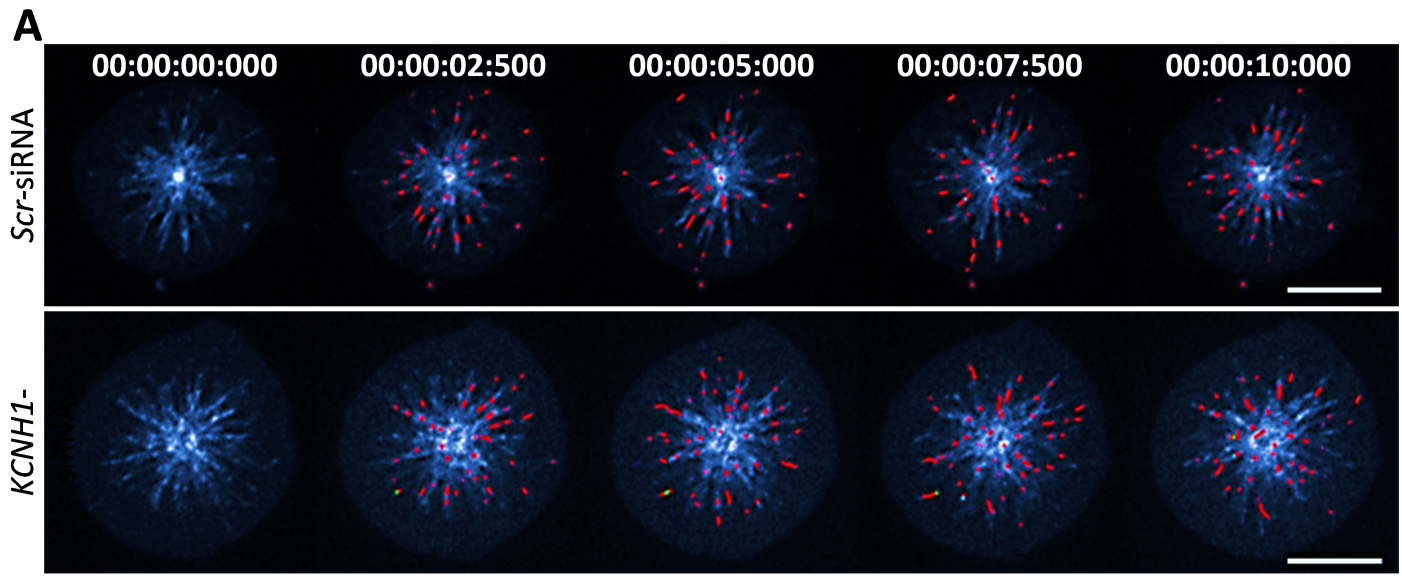

B

HeLa cells
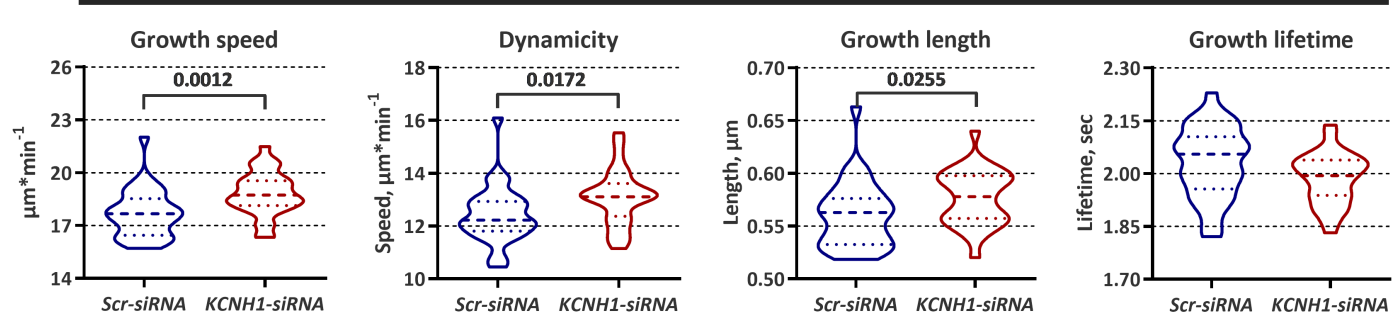

C

hTERT RPE1 cells
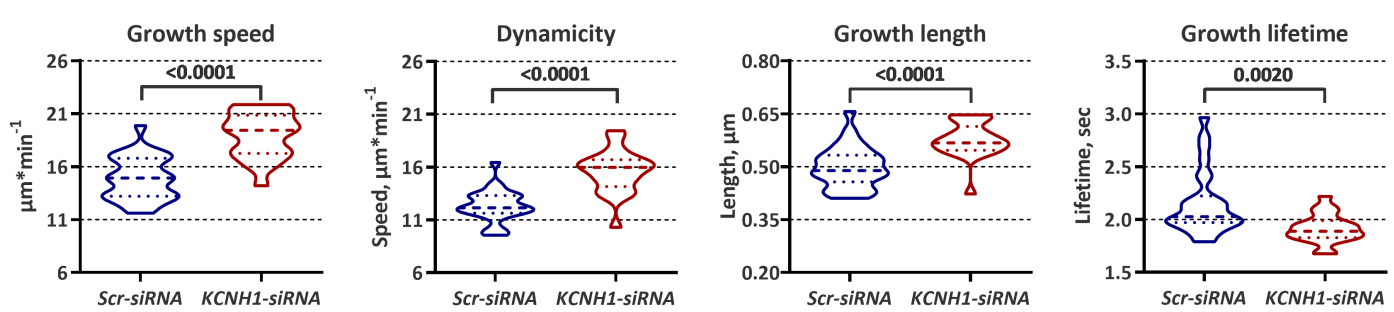

Figure 3.8: Increased microtubule plus-end growth rates are observed upon Kv10.1 downregulation. The cells were transiently co-transfected with the respective siRNAs and $p E B 3-$ tdTomato and treated $48 \mathrm{~h}$ post-transfection with $2.5 \mu M$ DME for $4 \mathrm{~h}$. The MT growth was tracked by taking images every $500 \mathrm{~ms}$ for $1 \mathrm{~min}$, and analysed with the MATLAB-based U-Track software (Jaqaman et al., 2008). A, The images represent constitutive series of analysed videos, where red "comets" mark length increase from lapse to lapse. Parameters calculated by the software are plotted in B, for HeLa and in C, for hTERT RPE1 cells (25 mitotic cells per experiment per condition, $n$ $=3$ independent synchronisation experiments; unpaired two-tailed Student's t-test, median and 25 and 75 percentiles are shown).

significant, whereas in hTERT RPE1 cells with silenced Kv10.1 (1.91 $\pm 0.03 \mathrm{~s})$ it was decreased by $0.23 \mathrm{~s}$ compared to the control cells $(2.15 \pm 0.05 \mathrm{~s})$.

Furthermore, dynamicity, the frequency rate of switching from growth to shrinkage and vice-versa (Burbank \& Mitchison, 2006), was measured. The MTs were more dynamic in both cell lines when Kv10.1 was downregulated. In HeLa cells, the difference in dynamicity of MTs between KD (13.08 $\left.\pm 0.19 \mu \mathrm{m} * \mathrm{~min}^{-1}\right)$ and control cells $\left(12.42 \pm 0.19 \mu \mathrm{m} * \mathrm{~min}^{-1}\right)$ was $0.67 \mu \mathrm{m} * \mathrm{~min}^{-1}$. In hTERT RPE1 KD $(15.57 \pm$ 
$\left.0.45 \mu \mathrm{m} * \mathrm{~min}^{-1}\right)$ and control cells $\left(12.31 \pm 0.29 \mu \mathrm{m} * \mathrm{~min}^{-1}\right)$ it was $3.26 \mu \mathrm{m} * \mathrm{~min}^{-1}$.

In conclusion, Kv10.1 downregulation leads to increased MT dynamics both in HeLa and hTERT RPE1 cells, thus confirming the results obtained from DME-assay.

\subsubsection{Overexpression of Kv10.1 has opposite effects to}

\section{Kv10.1 knockdown on MT dynamics in HeLa cells}

In order to understand whether the observed increase in MT dynamics is, indeed, due to the lack of Kv10.1, we repeated the MT dynamics experiment overexpressing WT Kv10.1 (pKCNH1-WT) in HeLa cells. As a control, cells were transfected with an empty plasmid with the same backbone used for pKCNH1-WT.

Overexpression of Kv10.1 in HeLa cells affected the MT dynamics (Figure 3.9, supplementary video $5-6$ ) opposite to the downregulation of the channel (Figure 3.8, B). The growth speed in cells overexpressing the channel was decreased from $17.56 \pm$ $0.15 \mu \mathrm{m} * \mathrm{~min}^{-1}$ (pEmpty) to $16.73 \pm 0.15 \mu \mathrm{m} * \mathrm{~min}^{-1}$ (pKCNH1-WT). The growth length was also reduced to $0.61 \pm 0.01 \mu \mathrm{m} * \mathrm{~min}^{-1}$ in pKCNH1-WT expressing cells relative to the control cells $\left(0.64 \pm 0.01 \mu \mathrm{m} * \mathrm{~min}^{-1}\right)$. In agreement with the findings upon downregulation, overexpression of Kv10.1 had no effect on growth lifetime in HeLa cells. Finally, the MTs in the cells with upregulated Kv10.1 were less dynamic $\left(14.48 \pm 0.18 \mu \mathrm{m} * \mathrm{~min}^{-1}\right)$ than the control cells $\left(15.28 \pm 0.23 \mu \mathrm{m} * \mathrm{~min}^{-1}\right)$.

The results described up to now lead us to propose that the delay of G2/M and activation of SAC are mainly due to the effects Kv10.1 has on MT dynamics.

HeLa cells
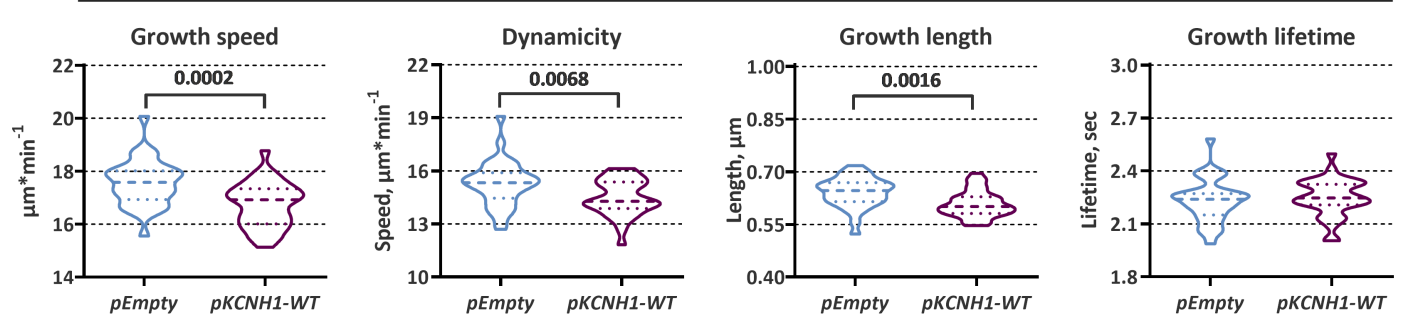

Figure 3.9: Overexpression of Kv10.1 in HeLa cells results in suppressed MT dynamics. HeLa cells were transiently co-transfected with respective $p D N A$ and $p E B 3-t d T o m a t o$, and 48 post-transfection treated with $2.5 \mu M D M E$ for $4 \mathrm{~h}$. The MT growth was tracked by taking images every $500 \mathrm{~ms}$ for $1 \mathrm{~min}$, and the MT growth was analysed with MATLAB-based U-Track software (Jaqaman et al., 2008). Parameters calculated by the software are plotted (25 mitotic cells per experiment per condition, $n=3$ independent synchronisation experiments; unpaired two-tailed Student's t-test, median and 25 and 75 percentiles are shown, $\left.{ }^{*} P=0.05\right)$. 
Although the differences we observe are quantitatively small, it is important to keep in mind that the experiments were performed using transient downregulation or overexpression of the channel, where the efficiency is subject to variability from experiment to experiment.

\subsection{Kv10.1 and ORAI1 functional interaction}

\subsubsection{Kv10.1 and ORAI1 are in close proximity to each other}

Until this point, the canonic function of the channel as a pathway for the flow of $\mathrm{K}^{+}$ions has not been taken into account. It could however have a relevant role in modifying the microenvironment by changing the ionic composition in a given cell compartment. In particular, the influence of different ions on the polymerisation of MTs in vitro and in vivo has been intensively studied (reviewed in Hepler, 2016; Safinya et al., 2016). Of special interest, although, are $\mathrm{Ca}^{2+}$ ions. MTs switch from growth phase to shrinkage when exposed to a high concentration of $\mathrm{Ca}^{2+}$ (Nishida et al., 1979; Sandoval \& Weber, 1978). However, the effect is highly dependent on the set of MAPs present on the growing tips of MTs (Berkowitz \& Wolff, 1981; V. Buljan et al., 2009). Furthermore, CaM has also been described to influence the growth rate of MTs (Erneux et al., 1984; Y. C. Lee \& Wolff, 1982). In addition, it has recently been suggested that Kv10.1 colocalises with ORAI1 based on fluorescence microscopy, and silencing of Kv10.1 or ORAI1 reduced $\mathrm{Ca}^{2+}$ influx, but without an additive effect, indicating a functional coupling between the channels (Badaoui et al., 2018).

Therefore, we checked the possible functional colocalisation of Kv10.1 and ORAI1 using PLA. If two proteins are in proximity, PLA probes bound to each of the target proteins will be able to interact with the connector oligonucleotide and start the RCA. As shown in Figure 3.10, A, PLA using Kv10.1 and ORAI1 antibodies gave rise to a signal, indicating proximity. There was no signal in the negative control for the assay (no primary antibody added; Figure 3.10, B). Thus, we could confirm that the previous indications using immunofluorescence approaches (Badaoui et al., 2018) obey physical proximity between the two channels. 
A

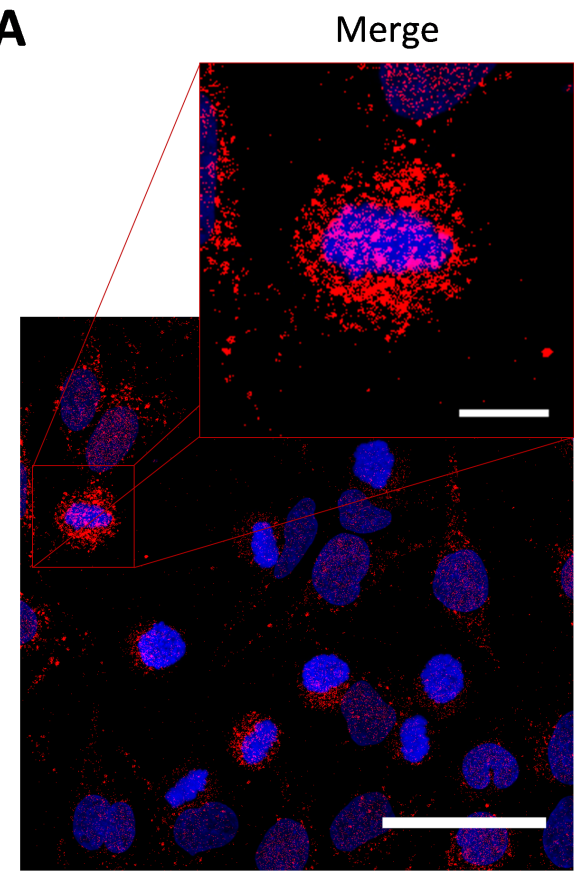

Kv10.1/ORAI1 PLA

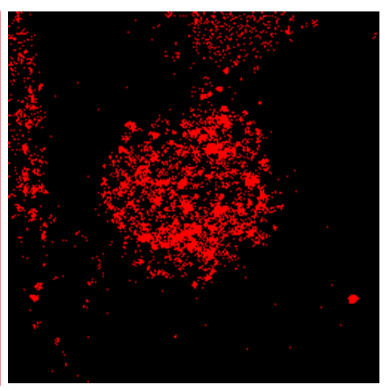

Nuclei

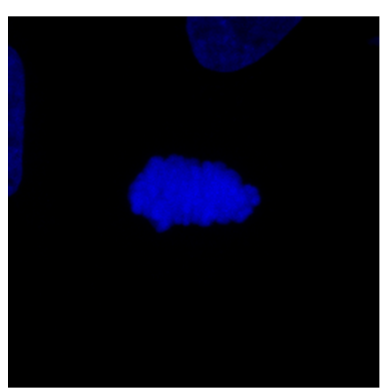

B

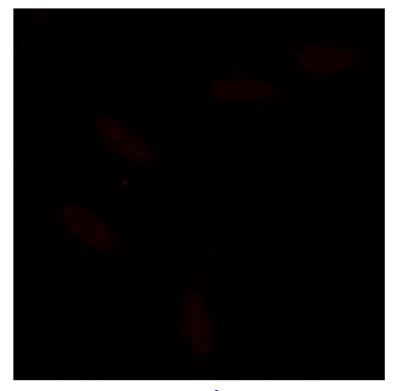

Nuclei

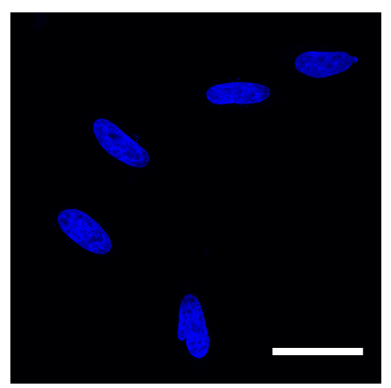

Figure 3.10: Kv10.1 and ORAI are in close proximity. Asynchronously growing HeLa cells were fixed with 2\% pfa in PHEM, amd ORAI1 and Kv10.1 colocalisation was checked using in situ proximity ligation assay: a positive signal can only be detected when both proteins are in close proximity. A, Example images showing Kv10.1/ORAI1 PLA foci in asynchronous cells (scale bar, $50 \mu \mathrm{m})$ and in a mitotic cell (inlet, scale bar $10 \mu \mathrm{m})$ are given (Kv10.1/ORAI1 PLA - red, nuclei blue). B, A negative control (no primary antibodies) is represented (scale bar, $50 \mu \mathrm{m}$ ).

\subsubsection{Silencing of Kv10.1 decreases the intracellular $\left[\mathrm{Ca}^{2+}\right]$ in hTERT RPE1 cells}

We showed that these two channels are in proximity, giving rise to the possibility that Kv10.1 activity results in an increase of $\left[\mathrm{Ca}^{2+}\right]$ through ORAI1. This would give rise to a self-limited loop since the increase of $\left[\mathrm{Ca}^{2+}\right]$ leads to closing of $\mathrm{Kv} 10.1$ through calcium/CaM (Ziechner et al., 2006).

To determine the presence of such a functional interaction, we measured changes of intracellular $\left[\mathrm{Ca}^{2+}\right]$ after silencing Kv10.1 in hTERT RPE1 cells. hTERT RPE1 cells were synchronised in G0 phase and preloaded with Fluo-4AM. The cells were first imaged in the absence of FCS for $60 \mathrm{~min}$, then FCS was added and the Fluo- 4 signal was immediately recorded from the same cells for another $60 \mathrm{~min}$ (Figure 3.11, A, supplementary video $7-10)$. The recorded signal was normalised for photobleaching and for continuous slow loading of the cells with Fluo-4AM, which was present throughout the imaging (Figure 3.11, B). The area under the curve (AUC) and the number of local maxima in the spectrum were calculated. As shown in Figure 3.11, 
A
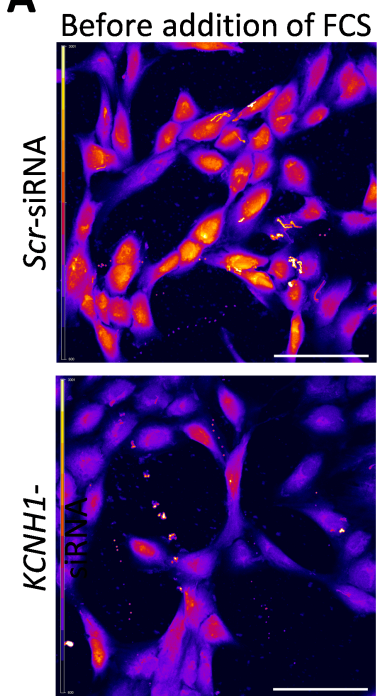

C

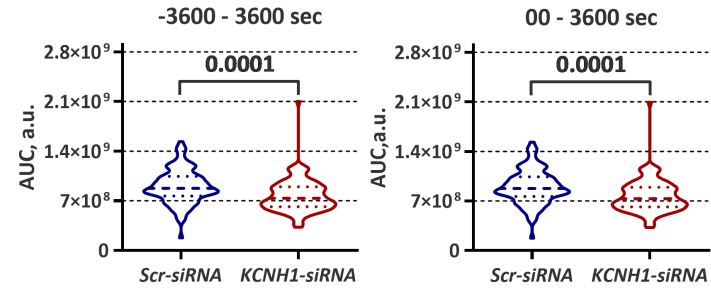

B
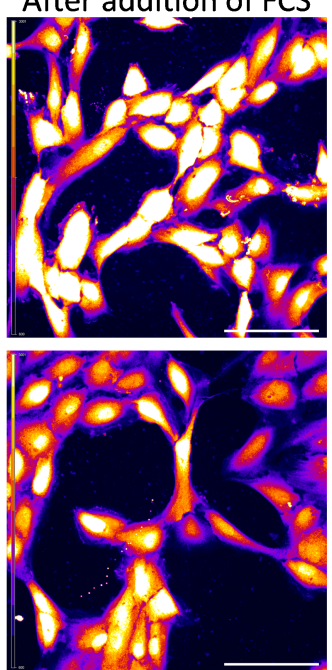

D
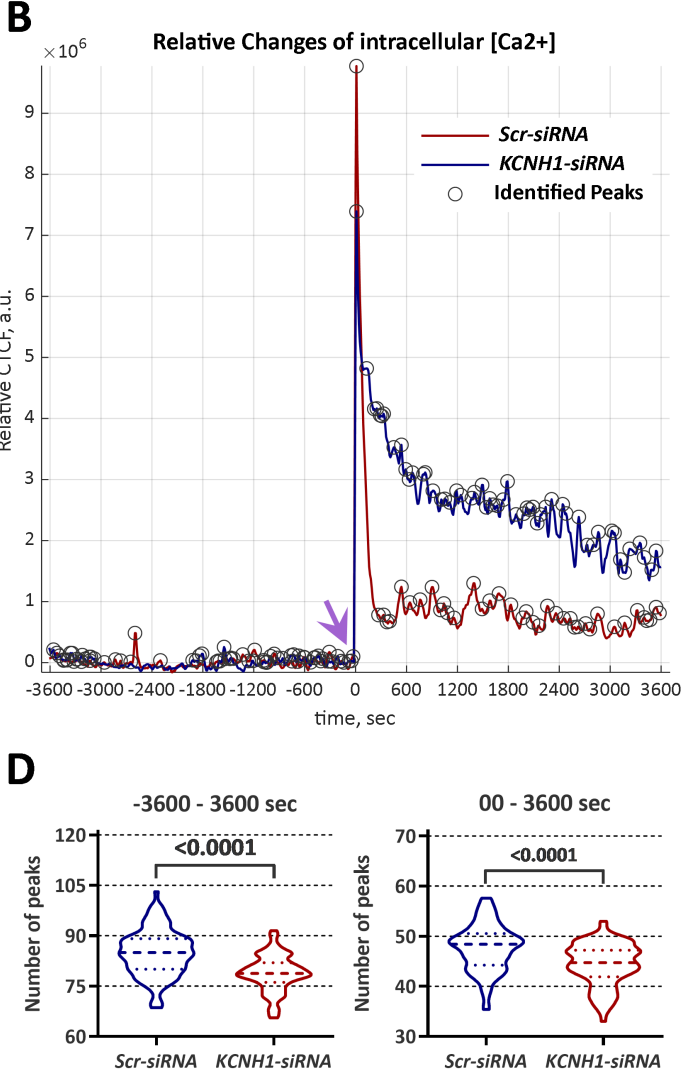

$00-3600 \mathrm{sec}$

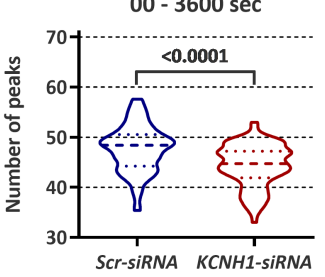

Figure 3.11: Kv10.1 downregulation decreases the intracellular $\left[\mathrm{Ca}^{2+}\right]$ and spiking in hTERT RPE1 cells. hTERT RPE1 cells were synchronised at G0 with serum deprivation protocol, and prior to the imaging, the cells were incubated with Fluo-4AM. The time-lapse images were taken with $10 \mathrm{~s}$ interval, first, in the absence of growth factors $(-3600-00 \mathrm{sec})$, then continued after addition of FCS $(00-3600 \mathrm{sec})$, and changes in the intensity of Fluo-4 were recorded. A, Maxima projection images of time-lapse series of Scr-and KCNH1-siRNA treated cells are shown before and after FCS addition with colour-coded intensity of Fluo-4 ("red fire" LUT is applied, scale bar, $100 \mu \mathrm{m})$. B, The measured intensity was normalised for bleaching and the relative changes in Fluo-4 intensity are plotted over time. An example of normalised spectra with identified local maxima (peaks, marked with circles) are given (the arrow marks the time of FCS addition). C and $D$, The total area under the recorded spectrum $(C)$ and number of peaks $(D)$ were determined for the entire duration and after FCS addition with MATLAB and analysed with GraphPad Prism 8 (200 cells per condition per experiment; $n=3$ independent synchronisation experiments; unpaired two-tailed Student's t-test, median and 25 and 75 percentiles are shown, $\left.{ }^{*} P=0.05\right)$.

$\mathrm{C}$, the mean AUC, which indicates the relative amount of intracellular $\left[\mathrm{Ca}^{2+}\right]$, was significantly smaller in KCNH1-treated cells: by $17 \%$ both for the entire duration $(-3600-3600 \mathrm{sec})$ and after addition of FCS $(00-3600 \mathrm{sec})$ respective to the ScrsiRNA treated cells. The total number of local maxima (peaks) was decreased by $8 \%$ both for the entire period and after stimulation with FCS in Kv10.1 KD cells (Figure 3.11, D). 


\subsubsection{Loss of Kv10.1 reduces the frequency of cytosolic calcium oscillations}

It is well established that calcium signals are not only encoded in the absolute magnitude of changes but also the frequency and amplitude of cytosolic calcium oscillations (Parekh, 2011; Samanta \& Parekh, 2017). In the previous section, we showed a decrease in total intracellular $\left[\mathrm{Ca}^{2+}\right]$ and also in the number of peaks in the intensity spectrum upon downregulation of Kv10.1. In particular, the latter may indicate a potential change in the oscillatory behaviour of cytosolic calcium upon Kv10.1 loss.

Therefore, using the same normalised recordings described in Figure 3.11, we calculated the changes in the frequency by converting the time domain of the signal into the frequency domain using fast Fourier transform (Figure 3.12, A). Four regions were isolated for the analysis: i) "before" (-3600 - 00 sec) and ii) "after" (00 - 3600 sec) FCS addition; iii) shortly after addition of FCS, the prominent peak region $(00-60 \mathrm{sec})$ and iv $)$ the region "after the peak" (60 - $3600 \mathrm{sec})$. As depicted in Figure 3.12, B, no significant differences were found between the control and KD cells in "before", unlike the "after" FCS region. That is, Kv10.1 loss did not affect the behaviour of $\mathrm{Ca}^{2+}$ oscillations at rest. The frequency of cytosolic calcium oscillations was decreased by almost $40 \%$ in the "after" FCS region of Kv10.1-depleted cells $(0.37 \pm 0.02 \mathrm{mHz})$ relative to the control group $(0.59 \pm 0.04 \mathrm{mHz})$.

The frequency rates in the "peak" region were $3.97 \pm 0.15 \mathrm{mHz}$ for Kv10.1 KD and $5.01 \pm 0.28 \mathrm{mHz}$ for the control hTERT RPE1 cells. The frequency rates in the "after the peak" were $2.17 \pm 0.16 \mathrm{mHz}$ and $2.72 \pm 0.16 \mathrm{mHz}$, respectively.

Next, we studied in more detail the properties of the frequency signal. We measured the prominence and FWHM of each local maximum within the frequency domain (Figure 3.13, A). These parameters give an idea about the strength of each spike (prominence) and its duration (FWHM). Three regions were analysed: i) entire duration (-3600 - $3600 \mathrm{sec})$; ii) after FCS addition (00 - $3600 \mathrm{sec})$ and iii) the "peak" (00 - 60 sec, Figure 3.13, B).

Globally, the signal strength in Kv10.1 depleted cells was reduced. The prominence and FWHM were reduced by $13 \%$ and $5 \%$, respectively, in the KD cells in comparison with the control group. After FCS addition, no significant change was 
A Single-Sided Amplitude Spectrum of relative changes of intracellular $[\mathrm{Ca2+}](\mathrm{t})$

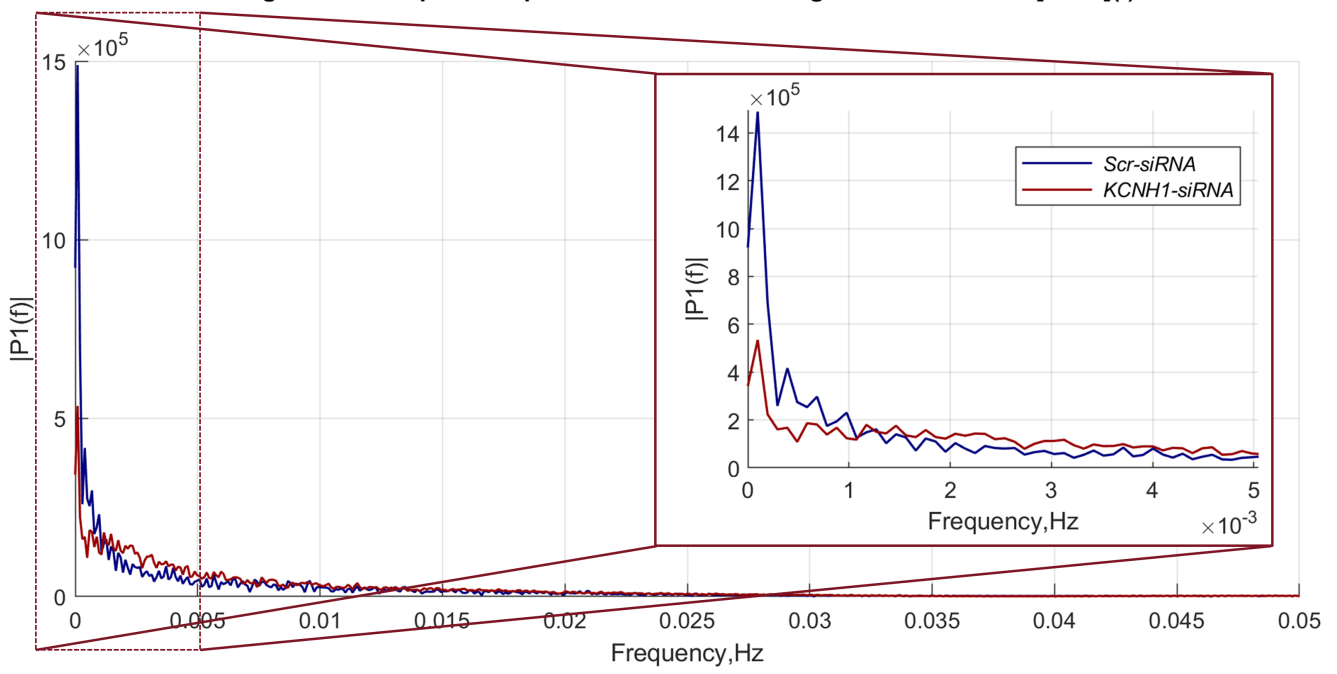

B

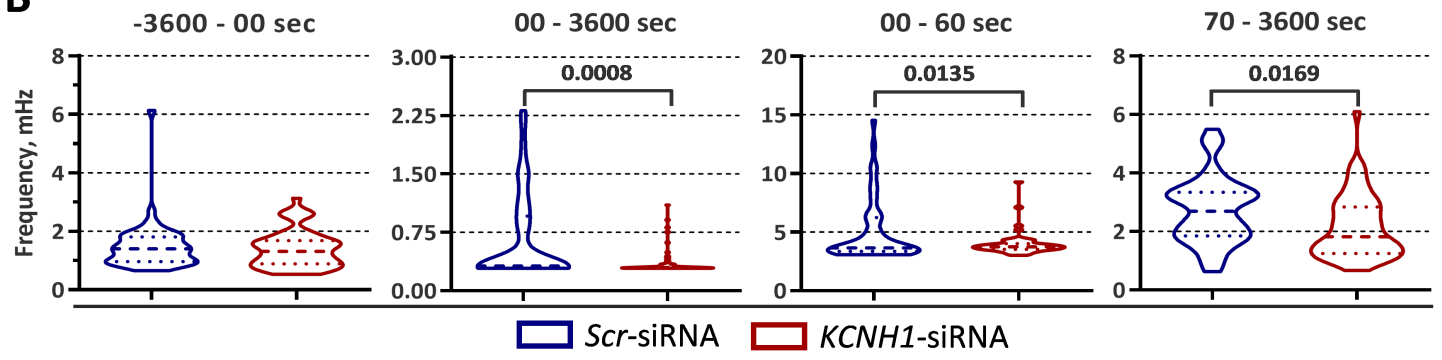

Figure 3.12: Kv10.1 downregulation decreases the frequency rate of intracellular $\left[\mathrm{Ca}^{2+}\right]$ oscillations. hTERT RPE1 cells were synchronised at GO with serum deprivation, and prior to the imaging, the cells were incubated with Fluo-4AM. The time-lapse images were taken with $10 \mathrm{~s}$ intervals, first, in the absence of growth factors (-3600 - $00 \mathrm{sec})$, then continued after addition of FCS (00 - $3600 \mathrm{sec})$. The recorded changes of intensity of Fluo-4 over time were converted into a frequency domain. A, An example of single-sided amplitude spectrum of relative changes of intracellular $\left[\mathrm{Ca}^{2+}\right]$ from one cell/condition recording is shown. B, The frequency was determined for the given regions ("00 sec" marks the point of FCS addition) with MATLAB and analysed with GraphPad Prism 8 (200 cells per condition per experiment; $n=3$ independent synchronisation experiments; unpaired two-tailed Student's t-test, median and 25 and 75 percentiles are shown, ${ }^{*} P$ $=0.05$.

observed in FWHM values, but the mean prominence was again smaller by $10 \%$ in the KD cells. Interestingly, the strength of the peak observed right after the addition of FCS (Figure 3.13, B, $00-60 \mathrm{sec}$ ), was affected by the downregulation of Kv10.1 the most $-20 \%$, however the FWHM was reduced moderately, only by $3 \%$.

In conclusion, here we give indirect evidence of functional interaction of Kv10.1 and ORAI1: silencing of Kv10.1 leads to the reduction in frequency, strength and duration of oscillations of cytosolic calcium, which is reflected in the global decrease of intracellular $\left[\mathrm{Ca}^{2+}\right]$. In addition, based on our initial findings on MT dynamics in Kv10.1-downregulated cells, which display increased growth speed and dynamicity, 

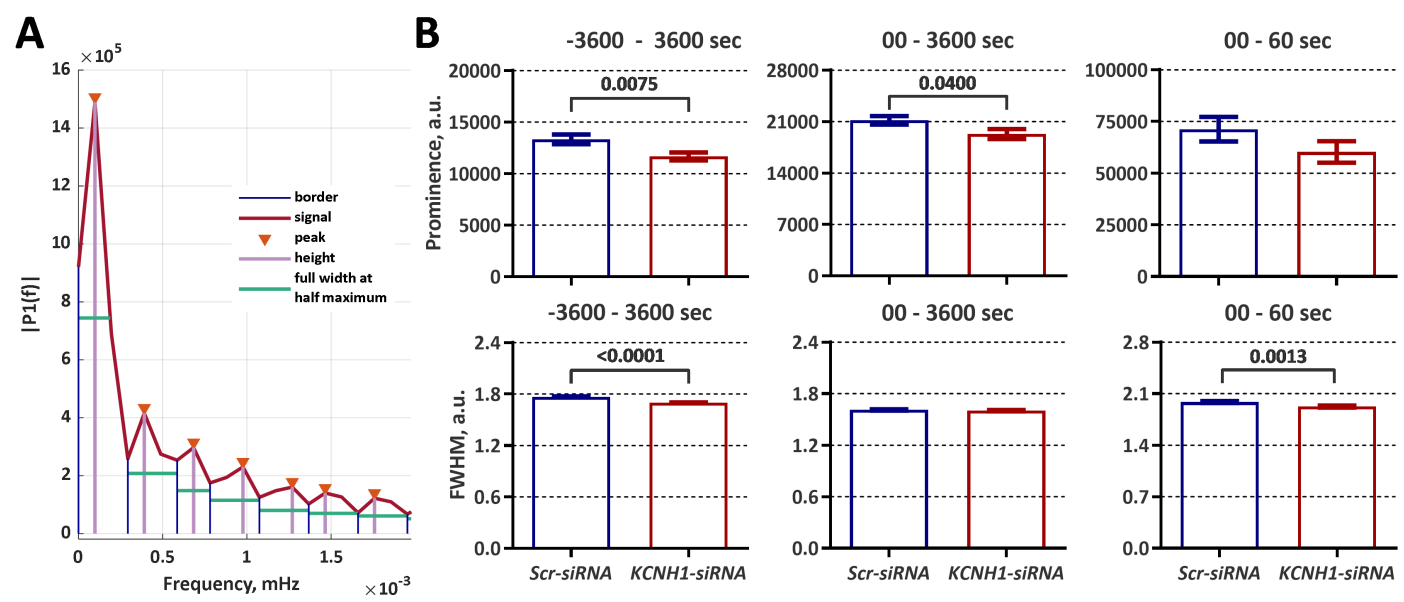

Figure 3.13: Weaker spikes of intracellular $\left[\mathrm{Ca}^{2+}\right]$ are observed upon the loss of Kv10.1 in hTERT RPE1 cells. The recorded changes of intensity of Fluo-4 over time were converted into a frequency domain. The prominence and full width at half maximum (FWHM) were defined in the single-sided amplitude spectrum with MATLAB as shown in A for the given regions ("00 sec" marks the point of FCS addition). B, The obtained values were analysed with GraphPad Prism 8 (200 cells per condition per experiment; $n=3$ independent synchronisation experiments; unpaired two-tailed Student's t-test, median and 25 and 75 percentiles are shown, $\left.{ }^{*} P=0.05\right)$.

global decrease of intracellular $\left[\mathrm{Ca}^{2+}\right]$ was expected; as already mentioned the polymerisation rate of MTs is negatively correlated with $\left[\mathrm{Ca}^{2+}\right]$, at least in vitro (Nishida et al., 1979; Sandoval \& Weber, 1978).

\subsection{Importance of conductance of $\mathrm{Kv10.1}$ and \\ ORAI1 in the regulation of MT dynamics}

\subsubsection{Astemizole-mediated blockade of Kv10.1 conductance reduces MT dynamics in HeLa cells}

In order to see whether the already described effects of Kv10.1 downregulation on MT dynamics are explicitly due to its conductance, rather than interactions of the cytoplasmic domains of the full-length Kv10.1, as well its splice-variants with other proteins, experiments involving specific blockers of the channel should be performed. An antihistamine drug astemizole has been described to specifically block Kv10.1 current (García-Ferreiro et al., 2004b). Therefore, we decided to utilise the same approach as before and study the effect of blockade of Kv10.1 conductance with the antihistamine drug astemizole on MT dynamics. 

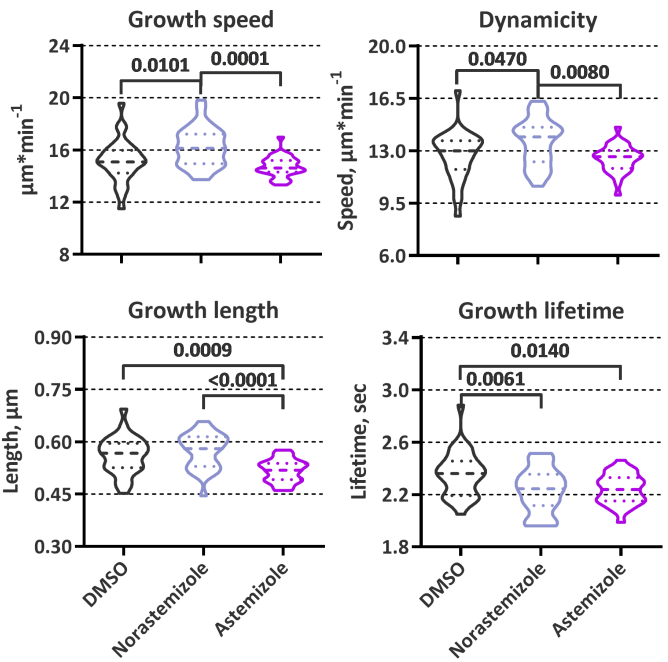

Figure 3.14: Blocking Kv10.1 conductance in HeLa cells with astemizole rescues the effect of norastemizole on MT dynamics. HeLa cells, transiently expressing EB3tdTomato, $24 \mathrm{~h}$ prior to the imaging were incubated with $10 \mu \mathrm{M}$ of either astemizole or norastemizole and the last $4 \mathrm{~h}-$ with $2.5 \mu M$ DME. The MT growth was tracked by taking images every 500 ms for 1 min, and analysed with MATLAB-based U-Track software (Jaqaman et al., 2008). Parameters calculated by the software are plotted (30 mitotic cells per experiment per condition, $n=$ 3 independent synchronisation experiments; oneway ANOVA, multiple comparison Tukey's test; median and 25 and 75 percentiles are shown, ${ }^{*} P$ $=0.05)$.

The growth speed was also higher in the norastemizole $\left(16.22 \pm 0.27 \mu \mathrm{m} * \mathrm{~min}^{-1}\right)$ vs the DMSO $\left(15.18 \pm 0.30 \mu \mathrm{m} * \mathrm{~min}^{-1}\right)$ or astemizole $\left(14.72 \pm 0.14 \mu \mathrm{m} * \mathrm{~min}^{-1}\right)$ treated cells. The MTs were shorter living in norastemizole $(2.230 \pm 0.03)$ than the DMSO $(2.356 \pm 0.03)$ treated cells, but we observed no changes between the astemizole and norastemizole groups. Interestingly, no significant changes in growth length were detected between norastemizole and DMSO-treated groups, whereas the astemizole effect on the growth length was notable - it was shorter by 0.045 $\mu \mathrm{m}$ and by $0.057 \mu \mathrm{m}$ relative to DMSO and norastemizole groups, respectively. No differences in growth lifetime were found between the astemizole and norastemizole groups. 
In summary, astemizole treatment had no noticeable effects with respect to the DMSO group, but MT dynamics were remarkably different between the astemizole and norastemizole groups. Astemizole interacts not only with the histamine receptor but also cytochrome P450 (Renwick, 1999). Importantly, the effects of astemizole were opposite to those of Kv10.1 downregulation and similar to Kv10.1 overexpression.

The effects of astemizole on cell physiology are pleiotropic, and reducing the difference between astemizole and norastemizole to only channel inhibition may be an oversimplification. However, our data indicate that blocking Kv10.1 conductance decreases the MT dynamics, similarly to overexpression of the channel (Figure 3.9), and opposite to its downregulation (Figure 3.8). Given this unexpected result, we set out for experiments using a more specific channel blocker.

\subsubsection{Monoclonal antibody-mediated blockade of Kv10.1 conductance decreases MT dynamics in HeLa cells}

The interpretation of norastemizole and astemizole effect on the MT dynamics is ambiguous. Therefore, we approached this question from another perspective: instead of a chemical agent, we used a monoclonal antibody (mAb56) directed against the pore loop of Kv10.1. mAb56 has been shown to inhibit Kv10.1 currents with high specificity and effectiveness (Gomez-Varela et al., 2007). Analysis of the timelapse series showed a decrease in MT dynamics in mAb56-treated cells (Figure 3.15, supplementary video $14-15)$. The growth speed in the mAb56 cells $(18.01 \pm 0.08$ $\left.\mu \mathrm{m} * \mathrm{~min}^{-1}\right)$ was decreased by $0.51 \mu \mathrm{m} * \mathrm{~min}^{-1}$ versus the isotype IgG-treated control group $\left(18.52 \pm 0.11 \mu \mathrm{m} * \mathrm{~min}^{-1}\right)$. The effect of mAb56 treatment on growth length and lifetime was insignificant. However, the cells treated only with IgG displayed more dynamic MTs $\left(16.34 \pm 0.11 \mu \mathrm{m} * \mathrm{~min}^{-1}\right)$ than the mAb56 treated cells $(15.83$ $\left.\pm 0.08 \mu \mathrm{m} * \mathrm{~min}^{-1}\right)$.

These results confirm the role of Kv10.1 currents in the regulation of MT dynamics, although opposite to the downregulation of the channel. To a certain extent, the blockade with antibody repeats the pattern of MT dynamics in the presence of astemizole. Nevertheless, it is clear that both the conductance of Kv10.1 and the presence of the actual channel is important for maintaining proper mitotic dynamics. 

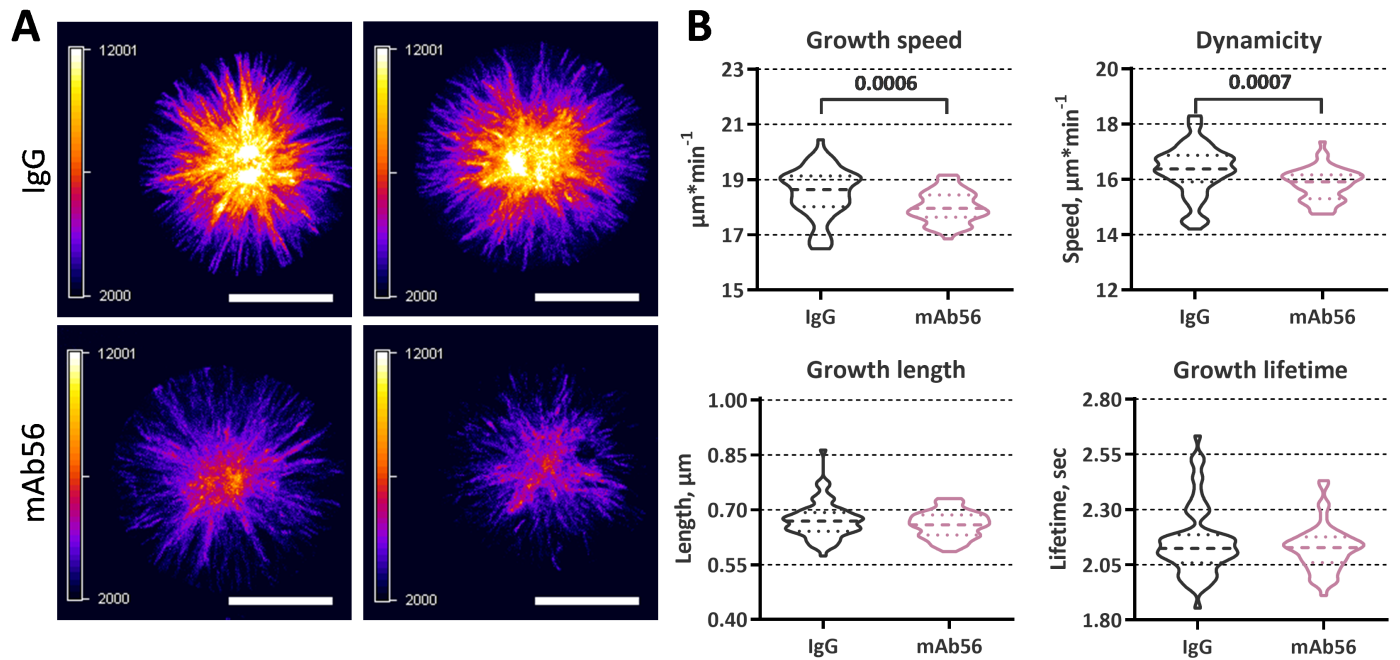

Figure 3.15: Blocking Kv10.1 conductance in HeLa cells with mAb56 decreases MT dynamics. HeLa cells, transiently expressing EB3-tdTomato, $24 \mathrm{~h}$ prior to the imaging were incubated with $5 \mu \mathrm{g}$ of either $m A b 56$ or $\operatorname{Ig} G(\kappa 2 b)$ and the last 4 - with $2.5 \mu M D M E$. The MT growth was tracked by taking images every $500 \mathrm{~ms}$ for 1 min, and the MT growth was analysed with MATLAB-based U-Track software (Jaqaman et al., 2008). A, The images represent maxima intensity projection of time-lapse series of EB3-tdTomato expressing cells with colour-coded intensity (scale bar, $10 \mu \mathrm{m}$ ). B, Parameters calculated by the software are plotted (50 mitotic cells per experiment per condition, $n=5$ independent synchronisation experiments; unpaired two-tailed Student's t-test, median and 25 and 75 percentiles are shown).

\subsubsection{Inhibition of ORAI1 conductance mimics the effects}

\section{of inhibition of Kv10.1 current on MT dynamics}

After confirming the physical proximity of Kv10.1 and ORAI1, a logical question would be whether the effects on MT dynamics observed by inhibiting Kv10.1 are due to this interaction, that is, that Kv10.1 affects MTs through a change in intracellular $\left[\mathrm{Ca}^{2+}\right]$ due to modulation of ORAI1 activity. Hence, we used AnCoA4, a drug known to inhibit ORAI1 current (Sadaghiani et al., 2014).

HeLa cells were transiently transfected with EB3-tdTomato and, prior to imaging, incubated with AnCoA4 and DME for $4 \mathrm{~h}$ (Figure 3.16, A, supplementary video 16 - 17). Analysis of the time-lapse series showed that MT dynamicity was lower by $1.66 \mu \mathrm{m} * \mathrm{~min}^{-1}$ in the cells exposed to AnCoA4 $\left(13.31 \pm 0.14 \mu \mathrm{m} * \mathrm{~min}^{-1}\right)$ as compared to the DMSO-treated ones $\left(14.97 \pm 0.16 \mu \mathrm{m} * \mathrm{~min}^{-1}\right.$, Figure 3.16, B). The growth speed in these cells was decreased by $1.15 \mu \mathrm{m} * \mathrm{~min}^{-1}$ compared to the DMSO-treated cells $\left(16.90 \pm 0.13 \mu \mathrm{m} * \mathrm{~min}^{-1}\right)$. Similarly, the growth length was 

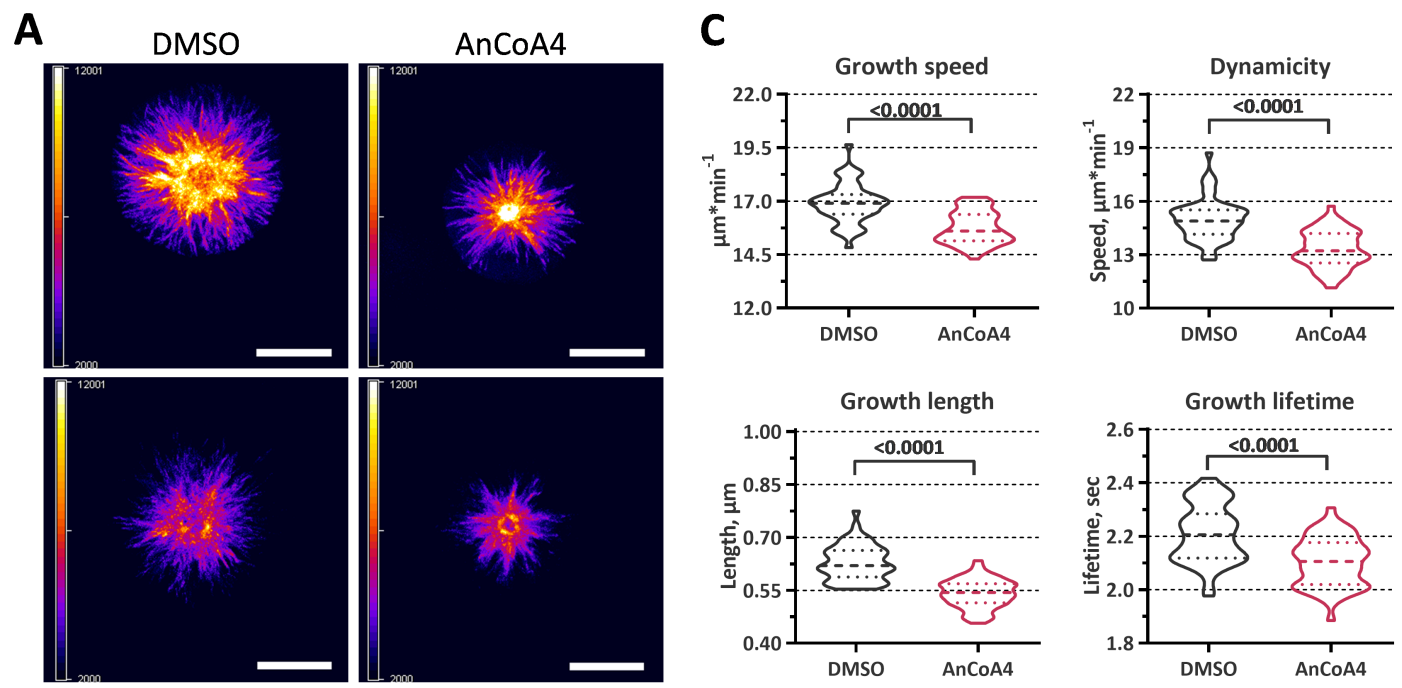

Figure 3.16: Blocking ORAI1 conductance in HeLa cells with AnCoA4 decreases MT dynamics. HeLa cells, transiently expressing EB3-tdTomato, $24 \mathrm{~h}$ prior to the imaging were incubated with $10 \mu M A n C o A 4$ or DMSO (equivalent volume), and the last 4h - with $2.5 \mu M D M E$. The MT growth was tracked by taking images every $500 \mathrm{~ms}$ for $1 \mathrm{~min}$, and the $M T$ growth was analysed with MATLAB-based U-Track software (Jaqaman et al., 2008). A, The images represent maxima intensity projection of time-lapse series of EB3-tdTomato expressing cells with colour-coded intensity (scale bar, $10 \mu \mathrm{m}$ ). B, Parameters calculated by the software are plotted (50 mitotic cells per experiment per condition, $n=3$ independent synchronisation experiments; unpaired two-tailed Student's t-test, median and 25 and 75 percentiles are shown).

shorter in AnCoA4 treated cells $(0.54 \pm 0.01 \mu \mathrm{m})$ than in the control group $(0.63 \pm$ $0.01 \mu \mathrm{m})$. The lifetime of growing MT tracks was shortened by $0.11 \mathrm{~s}$ relative to the control group (2.21 $\pm 0.02 \mathrm{~s})$.

In conclusion, we show here that MT behaviour is altered when ORAI1 current is inhibited. Moreover, this inhibition mimics the behaviour of MTs observed upon inhibition of Kv10.1 with mAb56 (Figure 3.15). Although the results of Kv10.1 knockdown and overexpression on one hand and inhibition of Kv10.1 and ORAI1 currents, on the other, are opposing each other, some proteins involved in the regulation of MT dynamics are described to have a dual effect (Cirillo, Gotta, \& Meraldi, 2017; Su et al., 2011). Most likely, the channel as well exerts a dual effect on the MT behaviour. It causes oscillations in intracellular $\left[\mathrm{Ca}^{2+}\right]$ through modulation the ORAI1 activity. At the same time besides the full-length Kv10.1, short splice variants of Kv10.1 resulting from exon-skipping events (E65 and E70) are present in human brain and cancer cell lines and can activate CDK1 (Ramos Gomes et al., 2015), which has been described to negatively regulate the MT growth rate (Fourest-Lieuvin et al., 2006). 
Results 


\section{Chapter 4}

\section{Discussion}

Prolonged and increased expression of Kv10.1, a voltage-gated potassium channel initially thought to be restricted to the central nervous system, has been detected in over $70 \%$ of all human tumour tissues. Presence of Kv10.1 in tumours correlates with poorer prognosis. Kv10.1 is known to be important for cell proliferation and angiogenesis. Recently, we showed that Kv10.1 is expressed in peripheral tissues, specifically during the G2/M phase of the cell cycle, and that downregulation of the channel extends the G2/M phase duration both in tumour and non-tumour cells (Urrego et al., 2016). Hence, this project aimed to elucidate the mechanisms of Kv10.1-mediated regulation at the G2/M phase. We studied the activation state of two checkpoints functioning in G2 and M phases.

\subsection{Kv10.1 knockdown results in the partial activation of DNA damage-induced checkpoint in the absence of DNA damage}

The main checkpoint mechanism throughout the entire cell cycle is the DNA damage-induced checkpoint. As already described in the "Introduction", the DNA damage-induced checkpoint utilises the DDR machinery. Therefore, we analysed the expression pattern of major proteins of DDR throughout the different phases of the cell cycle. Interestingly, we found that only the upper part of the pathway, ATR, CHK1 and CHK2, were activated in Kv10.1-depleted cells (Figure 3.1). Moreover, direct measurement of the level of DNA damage revealed no differences between the groups (Figure 3.2). Therefore, we conclude that the partial activation of DDR we observed should have other origins, rather than unresolved DNA lesions.

Interestingly, some DNA checkpoint proteins, including ATM, ATR, BRCA1, CHK1 and CHK2 are localised to centrosomes, a location compatible with their 


\section{DisCUSSION}

implication in the SAC (S. Zhang, Hemmerich, \& Grosse, 2007). ATM is needed for kinetochore localisation of MDC1 (Mediator of DNA damage checkpoint protein 1), and activation of MCC (Eliezer et al., 2014); ATM supports the activation of SAC also through phosphorylation of MAD1 (Yang et al., 2014).

Proper timing of the mitotic spindle formation has been linked to centrosomeassociated CHK1 by preventing the unscheduled activation of centrosomal CDK1 (Krämer et al., 2004). Moreover, it has been suggested that CHK1 is required for the optimal regulation of Aurora B (possibly through direct phosphorylation) and BUBR1 when kinetochores produce a weakened signal (Zachos et al., 2007). In good agreement with this, haploinsufficiency of CHK1 in mice causes multiple mitotic defects and enhances binucleation due to the mislocalisation of Aurora B (Peddibhotla, Lam, Gonzalez-Rimbau, \& Rosen, 2009). In U2OS cells, CHK1 depletion results in the failure to activate SAC and decrease of MAD2 and BUBR1 levels after spindle disruption by nocodazole (Carrassa, Sanchez, Erba, \& Damia, 2009). These studies suggest that CHK1 enhances the strength of SAC through direct effects on centrosomes or SAC proteins, such as Aurora B, and indirect effects through CDKs; CHK1 deficiency leads to mislocalisation of Aurora B, BUBR1 and MAD1 proteins, resulting in spindle checkpoint failure (Enders, 2008; Patil, Pabla, \& Dong, 2013).

Besides ATM and CHK1, a number of publications have reported the implication of CHK2 and BRCA1 in the regulation of mitotic events in the unperturbed cell cycle (Chabalier-Taste, Racca, Dozier, \& Larminat, 2008; Ertych, Stolz, Valerius, Braus, \& Bastians, 2016; Golan et al., 2010; Shang et al., 2014; Stolz et al., 2010). It has also been shown that MPS1 (monopolar spindle 1), a SAC protein that senses the unattached kinetochores, requires CHK2-mediated phosphorylation at Thr288 for the correct kinetochore localisation (Yeh, Yu, Chen, Cheng, \& Shieh, 2014).

In summary, in unperturbed cells, the role of DDR is broader than merely a mechanism for DNA lesions detection and repair. Therefore, upregulation of CHK2, CHK1 and ATR when Kv10.1 is downregulated can indicate the activation of SAC. 


\subsection{Kv10.1 downregulation enhances the SAC response and prevents the formation of lagging chromosomes}

As discussed above, the observed increase in the DDR proteins can be due to the alteration in the strength of SAC response. Indeed, analysis of the expression levels of SAC proteins in Kv10.1-deficient cells showed upregulation of some of them, such as MAD2, CDC20 and pVHL (Figure 3.3). MAD2 and CDC20 make up the MCC, a central effector of SAC (Poon, 2016). MCC sequesters CDC20 and thus prevents the activation of APC/C and delays the metaphase-to-anaphase transition (Musacchio \& Salmon, 2007). It is important to mention that the SAC response is graded, and it depends primarily on the amount of MAD2 protein present in a cell (Subramanian \& Kapoor, 2013). Hence, the increase of MAD2 along with CDC20 may induce a stronger SAC response in Kv10.1-depleted cells.

Another protein described to be involved in SAC is pVHL, mutation of which leads to von Hippel-Lindau (VHL) syndrome characterised by the presence of benign and malignant tumours (reviewed in Varshney et al., 2017). pVHL has been shown to reduce chromosomal instability through an increase of MAD2 levels (Hell et al., 2014; Thoma et al., 2009). We showed that Kv10.1 downregulation increases the levels of pVHL (Figure 3.3), thus supporting the idea of stronger SAC response in these cells.

Aurora B appears to occupy an upstream position in the pathway of recruitment of SAC components, as its inhibition prevents kinetochore recruitment of all other SAC components. Although total Aurora B was downregulated in Kv10.1-deficient cells (Figure 3.3), it is still possible that it is more abundant at the effective localisation, since Aurora B needs to be relocated at the kinetochores for exerting its CPC-related functions. MAD2 has been described to be crucial for Aurora B localisation at the kinetochores (Shandilya et al., 2016). CHK1 mediated phosphorylation of Aurora B increases its activity at centromeres (Zachos et al., 2007). We showed that both MAD2 and phosphorylated CHK1 are upregulated in Kv10.1 KD cells (Figure 3.3). Therefore, MAD2 and CHK1 may facilitate Aurora B localisation and activation at the kinetochores in these cells. Hence, although we observe a decrease in total Aurora 


\section{DisCUSSION}

B in Kv10.1-deficient cells, we can speculate that the amount of "effective" Aurora B may be higher in them. However, this hypothesis requires further investigation.

PLK1 is shown to support SAC in several ways. PLK1 phosphorylates MPS1 and enhances its catalytic activity as well as the recruitment of the SAC components MAD1/C-MAD2 and BUB3/BUBR1 to kinetochores (Ikeda \& Tanaka, 2017; von Schubert et al., 2015). Besides, PLK1 has been described to regulate the formation of proper kinetochore-MT attachments, generating tension between two sister chromatids (Shao et al., 2015). PLK1 colocalises with CLIP-170 at the kinetochores during early mitosis and ensures the maintenance of the kinetochore-MT stability (Amin, Itoh, Iemura, Ikeda, \& Tanaka, 2014). When PLK1 is inhibited, improper kinetochore-MT attachments are no longer resolved, resulting in abnormal anaphase with chromosome bridges (Shao et al., 2015). Even subtle loss in PLK1 activity have been described to impair chromosome congression (Lera \& Burkard, 2012). Overall, increased PLK1 activity upon Kv10.1 loss (Figure 3.5) can thus contribute to the strength of SAC by modulating the kinetochore-MT attachments and by facilitating the recruitment of MCC to kinetochores.

Mechanistically, inactivation of SAC requires the presence of the tension between the sister chromatids that is achieved by the formation of proper kinetochore-MT interactions in a process termed "error-correction" (Saurin, 2018). This ensures that all the chromosomes are correctly attached to the mitotic spindle in a bipolar fashion (May \& Hardwick, 2006). If SAC fails, then lagging chromosomes or chromosome bridges are observed during anaphase. We showed that the occurrence of such structures is less frequent in Kv10.1 KD cells, once again supporting the idea of enhanced SAC in these cells.

In addition to $\mathrm{SAC}$ activation, we also investigated the status of $\mathrm{APC} / \mathrm{C}$ activation. APC/C requires both binding of CDC20 to its subunit (King et al., 1995; Matyskiela \& Morgan, 2009; Peters, 2006) and hyperphosphorylation for full activation (S. Zhang et al., 2016). In vitro experiments have demonstrated that the APC/C complex can be phosphorylated by cyclin B1/CDK1 and PLK1 (Golan, Yudkovsky, \& Hershko, 2002). Interestingly, CDC20 itself is an APC/C substrate (Prinz, Hwang, Visintin, \& Amon, 1998). We show here that upon loss of Kv10.1 CDC27 protein is downregulated (Figure 3.3), while CDC20 is upregulated. Although, we observe 
high PLK1 activity in Kv10.1-depleted cells, it has been shown that PLK1 is not required for APC/C activation in vivo (Kraft et al., 2003), but rather the opposite. PLK1 is capable of preventing APC/C activation. Phosphorylation of CDC20 by BUB1-PLK1 inhibits APC/C(CDC20) activity in vitro and is required for checkpoint signalling in human cells (Ikeda \& Tanaka, 2017; Jia, Li, \& Yu, 2016). Therefore, we can conclude that APC/C activity is lower in Kv10.1 KD cells.

Nevertheless, the lack of CDC27 can be compensated by upregulation of other factors. A plausible candidate is WEE1, a kinase responsible for cyclin B1/CDK1 complex inactivation both at the entry into mitosis (Schmidt et al., 2017) and at the metaphase-to-anaphase transition (Chow et al., 2011). Overexpressing Swe1 (the paralog of WEE1 in budding yeast) results in the arrest of cells with reduced APC activity in metaphase in vitro and in vivo (Lianga et al., 2013). When we profiled WEE1 protein expression throughout the cell cycle progression, we observed upregulation of total WEE1 protein in Kv10.1-deficient cells (Figure 3.3, B). This upregulation of WEE1 may compensate for the decreased APC/C activity by taking over the inhibition of cyclin B1/CDK1 complex, thus triggering the metaphaseto-anaphase transition (reviewed in Schmidt et al., 2017; Visconti et al., 2012). Alternatively, WEE1 may prevent the premature activation of APC/C and consequently the formation of lagging chromosomes. Slightly broader peaks of CDC20 and WEE1 at G2/M phase ( 7 - $12 \mathrm{~h}$ after release from thymidine block), as well as our previous observations (Urrego et al., 2016) speak against the former possibility and hint to extended and stronger SAC activity and prevention of premature activation of $\mathrm{APC} / \mathrm{C}$.

In summary, we show that downregulation of Kv10.1 increases the protein level and activity of some of DDR and SAC regulators. This, in turn, results in a stronger SAC response and delays the metaphase-to-anaphase transition. The latter allows the cell to have more time for correcting erroneous kinetochore-MT attachments. 


\subsection{Kv10.1-deficient cells display tilted mitotic spindle orientation}

Interestingly, both PLK1 and pVHL are involved in the positioning of the mitotic spindle. The positioning of the mitotic spindle defines the angle of cell division, which is critical for cell differentiation, tissue organisation and morphology and EMT (epithelial-to-mesenchymal transition; Bergstralh \& St Johnston, 2014; Pease \& Tirnauer, 2011). pVHL promotes proper spindle orientation through stabilisation of astral MTs via interaction with kinesin-2 (Hell et al., 2014; Hergovich et al., 2002; Lolkema et al., 2007, 2004; Thoma et al., 2009). PLK1 promotes astral MT nucleation (Hanafusa et al., 2015; Miyamoto et al., 2017) and regulates the enrichment of cell cortex with dynein (Kiyomitsu \& Cheeseman, 2012; Sana et al., 2018), stabilising the spindle orientation. Since we observed an increase in both pVHL and PLK1 activity in Kv10.1-downregulated cells, a logical question was whether the lack of Kv10.1 affects the orientation of the mitotic spindle. Indeed, measurement of the mitotic spindle angle revealed larger values for Kv10.1-depleted cells (Figure 3.6). Interestingly, it is known that single HeLa cells with greater tilting of the mitotic spindle require more time for biorientation of sister kinetochores, which possibly results in the activation of SAC, while disruption of the spindle checkpoint activation does not correlate with the shape-dependent differences in spindle orientation (Charnley et al., 2013).

It is intriguing to find out whether Kv10.1 participates in positioning the mitotic spindle within tissue and so can influence the fate of the newly formed daughter cells. In particular, this is of great interest in the context of EMT, which is known to underlie metastasis (Aiello et al., 2018; Heerboth et al., 2015; Li \& Balazsi, 2018; Mittal, 2018; Reichert et al., 2018). Nevertheless, we can conclude that most probably the changes in the MT behaviour, which led to an alteration in mitotic spindle positioning, may be the cause for the observed increase in PLK1 and pVHL activities, and subsequently extended SAC. 


\subsection{Microtubule dynamics increase when Kv10.1 is downregulated}

Both SAC and spindle orientation are highly dependent on MT dynamics and can influence or be influenced by them (Kotak, 2019; Saurin, 2018; H.-Y. Wu et al., 2017). Therefore, we questioned whether the phenotypic and biochemical changes observed in Kv10.1-depleted cells could be attributed to the alterations in MT dynamics.

Preliminary assessment of the MT plus-end assembly rates using the DME assay (Stolz, Ertych, \& Bastians, 2015) showed that, as expected, the MT growth was affected upon loss of Kv10.1. Interestingly, the same approach has recently been proposed as a screening assay for determining mitotic spindle orientation changes (Glaubke \& Bastians, 2018). The authors observed a high correlation between cells harbouring defects in spindle orientation and the formation of asymmetric monopolar mitotic spindles. Thus, the increased number of asymmetric spindles observed in Kv10.1 KD cells in prometaphase (Figure 3.7) correlates also with the directly measured increased spindle angle in metaphase (Figure 3.6). These results were further confirmed by the assessment of MT dynamics in the live HeLa and hTERT RPE1 cells. Downregulation of Kv10.1 increased MT dynamics (Figure 3.8), whereas upregulation had an opposite effect (Figure 3.9), strongly suggesting that the effect is specific for Kv10.1.

Besides PLK1, whose role in assuring the needed stability of kinetochore-MT attachments we have already discussed, CHK2 also regulates MT dynamics. The CHK2-BRCA1 axis restrains oncogenic Aurora A activity during mitosis (Ertych et al., 2016). Downregulation of CHK2 or overexpression of Aurora A increases the assembly rate of MT plus-end (Ertych et al., 2014). In general, Aurora A promotes MT polymerisation and maintains MT flux ensuring proper bipolar spindle assembly (L.-h. Wang et al., 2008). Aurora A inhibition decreases MT shrinkage, growth rate, frequency rescue and nucleation (Lorenzo, Liao, Hardwicke, \& Ducommun, 2009). We show a strong upregulation of global CHK2, but not of phospho-Thr68 CHK2, needed for activation of BRCA1, in Kv10.1-deficient cells (Figure 3.1). However, since we have not checked for the corresponding phosphorylation of BRCA1, it is difficult to conclude whether the axis fails to modulate Aurora A activity in Kv10.1 


\section{DisCUSSION}

depleted cells.

Although we could not directly document changes in Aurora A protein expression, it is tempting to speculate that Aurora A activity is also altered in Kv10.1-depleted cells. There are several reasons for us to think so. Aurora A activation at the centrosomes is mediated by cyclin A2/CDK1-Bora axis, which also activates PLK1 (Asteriti, De Mattia, \& Guarguaglini, 2015; Macůrek et al., 2008; Macurek, Lindqvist, \& Medema, 2009; Parrilla et al., 2016; Thomas et al., 2016; Van Horn et al., 2010; Vigneron et al., 2018). Previously, we have reported an accumulation of cyclin A2 in Kv10.1-deficient cells (Urrego et al., 2016). Interestingly, one of the splice forms of Kv10.1, E65, found in human brain and cancer cell lines, activates CDK1 when injected into Xenopus oocytes (Ramos Gomes et al., 2015). Silencing of Kv10.1 will result in a reduction of both full length and the spliced forms of the channel, thus preventing E65-mediated CDK1 activation, and therefore reducing activation of Aurora A. On the other hand, here we reported an increase in PLK1. An intensive interplay between Aurora A and PLK1 exists, which can contribute to Aurora A activation at distinct subcellular sites (Asteriti et al., 2015). Nevertheless, this all together indicates that Aurora $\mathrm{A}$ activation at G2/M phase may be altered in Kv10.1-deficient cells, but this remains to be unequivocally documented.

Overall, the net activity of PLK1, Aurora B, pVHL, CHK2 and Aurora A contribute to the increased MT dynamicity in Kv10.1 KD cells. However, it is still unclear how the manipulation of a single channel can have such global effects.

\subsection{Kv10.1 and ORAI1 team up: downregulation of Kv10.1 reduces the cytosolic calcium concentration and oscillations}

In search of a global messenger which may be generated by Kv10.1, we looked at the question from another perspective. Until now, we had neglected the canonic function of the channel - a pathway for the flow of $\mathrm{K}^{+}$ions. Changing the ionic composition in a given cell compartment at a given time point can be the missing piece of the puzzle. Since the net activity of different proteins discussed before leads 
to specific MT behaviour, we decided to use it as a screening tool for finding the potential effector messenger. Different ions influence the polymerisation of MTs both in vitro and in vivo (reviewed in 2016;2016). $\mathrm{K}^{+}$promotes tubulin polymerisation in vitro, although the presence of MAPs diminishes the ion selectivity for the reaction (Wolff, Sackett, \& Knipling, 1996).

In terms of MT growth regulation, $\mathrm{Ca}^{2+}$ ions are of particular interest. MTs switch from growth phase to shrinkage when exposed to a high concentration of $\mathrm{Ca}^{2+}$ (Nishida et al., 1979; Sandoval \& Weber, 1978). Moreover, CaM has also been described to influence the growth rate of MTs (Erneux et al., 1984; Y. C. Lee \& Wolff, 1982). However, most importantly, it has recently been suggested that Kv10.1 colocalises with ORAI1 in immunofluorescence images, and that silencing of Kv10.1 and ORAI1 reduces $\mathrm{Ca}^{2+}$ influx without an additive effect, indicating a functional coupling between the channels (Badaoui et al., 2018).

Here, we tested the hypothesis of generating $\mathrm{Ca}^{2+}$ as the messenger by the coupled function of Kv10.1 and ORAI1, which could be responsible for the described phenotype. Indeed, using PLA, we directly showed the proximity of Kv10.1 and ORAI1, which may also imply the existence of a functional interaction between the two channels. Progression through different phases of the cell cycle appears to be regulated by transient changes in cytosolic $\left[\mathrm{Ca}^{2+}\right]$ (Kahl \& Means, 2003; Roderick \& Cook, 2008), in particular, during metaphase-to-anaphase transition (Kao, Alderton, Tsien, \& Steinhardt, 1990; Poenie, Alderton, Steinhardt, \& Tsien, 1986; Ratan, Maxfield, \& Shelanski, 1988; Tombes \& Borisy, 1989; Whitaker \& Larman, 2001). For example, injection of micromolar concentrations of $\mathrm{Ca}^{2+}$ accelerates the transition (Izant, 1983), whereas restriction of calcium flux from the extracellular compartment prolongs metaphase (Hepler, 1985). It is generally accepted that $\mathrm{K}^{+}$channels are important for $\mathrm{Ca}^{2+}$ influx. The hyperpolarisation generated by the activation of $\mathrm{K}^{+}$ channels increases the driving force for $\mathrm{Ca}^{2+}$ influx (Ouadid-Ahidouch \& Ahidouch, 2013). We tested this model for Kv10.1 and ORAI1 by silencing Kv10.1. If the activity of Kv10.1 is required for $\mathrm{Ca}^{2+}$ influx (not necessarily through ORAI1), then silencing it will reduce the cytosolic $\left[\mathrm{Ca}^{2+}\right]$. Indeed, downregulation of Kv10.1 decreased the total cytosolic $\left[\mathrm{Ca}^{2+}\right]$ in hTERT RPE1 cells after stimulation with FCS, and this decrease can explain the extended G2/M in Kv10.1-depleted cells 


\section{DisCUSSION}

(Urrego et al., 2016).

Mechanistically, changes in cytosolic $\left[\mathrm{Ca}^{2+}\right]$ can influence the metaphase-toanaphase transition in several ways. Earlier in the discussion, we suggested that APC/C might be less active in Kv10.1 KD cells, based on immunoblot analysis. Interestingly, the activation of $\mathrm{APC} / \mathrm{C}$ is blocked by $\mathrm{Ca}^{2+}$ chelators $(\mathrm{Xu}, \mathrm{Luo}, \&$ Chang, 2003). Therefore, when downregulating the channel, the cytosolic $\left[\mathrm{Ca}^{2+}\right]$ is also reduced and so is APC/C activity. Depletion of $\mathrm{Ca}^{2+}$ during mitosis also reduces kinetochore-MT stability due to the elimination of CENP-F from the kinetochore (Phengchat et al., 2016), which in the case of Kv10.1-depleted cells might be compensated by higher PLK1 activity. $\mathrm{Ca}^{2+} / \mathrm{CaM}$ has been shown to interact with and transiently activate Aurora A (Plotnikova, Pugacheva, Dunbrack, \& Golemis, 2010) both during mitosis and ciliary disassembly (Plotnikova et al., 2012). CaM also interacts and activates PLK1 in a calcium-dependent fashion during the G2/M transition (Dai et al., 2013). Since we show a decrease in total cytosolic $\left[\mathrm{Ca}^{2+}\right]$ in Kv10.1 depleted cells, decreased PLK1 activity could have been expected. However, we observe the opposite, meaning that possibly Kv10.1 KD leads to alternative ways of PLK1 activation (Asteriti et al., 2015; Lindqvist et al., 2009). Nevertheless, this further supports our suggested model. The phenotype of Kv10.1 KD cells, which consists of extended G2/M, stronger SAC, fewer lagging chromosomes and altered MT behaviour, can be adequately explained as the result of calcium transient changes, possibly generated by a teamwork of Kv10.1 and ORAI1.

Mounting evidence indicates that $\mathrm{Ca}^{2+}$ signals are often presented to cells in the form of $\mathrm{Ca}^{2+}$ oscillations, with signalling information encoded in both amplitude and frequency of the $\mathrm{Ca}^{2+}$ spikes (Parekh, 2011; Samanta \& Parekh, 2017). Different molecules have been described as calcium oscillation decoders. These decoders have defined affinity for $\mathrm{Ca}^{2+}$ binding, meaning that depending on the amplitude and the frequency of the signal only some of them will bind $\mathrm{Ca}^{2+}$, become activated and initiate a signalling pathway (reviewed in Smedler \& Uhlén, 2014). For example, activation of CaMKII is dependent on the frequency of cytosolic $\mathrm{Ca}^{2+}$ oscillations (Smedler \& Uhlén, 2014). Although our experimental set-up in terms of image acquisition rate was not optimal for exact determination of the frequency of oscillations, we were still capable of distinguishing that knockdown of Kv10.1 reduces the frequency 
and the amplitude of calcium oscillations in hTERT RPE1 cells when stimulated with FCS. It is appealing to think that Aurora A and PLK1 might react to the oscillations as well since both are activated by binding to calcium/CaM and serve as another example of signal decoders. This might also indicate a potential differential activation of any other given target sensitive to cytosolic calcium oscillations.

Overall, modulation of cytosolic $\left[\mathrm{Ca}^{2+}\right]$ in a functional complex with ORAI1 might be the missing piece of our puzzle that explains the extended duration of the G2/M phase with activated SAC and altered MT dynamics.

\subsection{Inhibition of the conductance of either}

\section{Kv10.1 or ORAI1 reduces MT dynamics}

Changes in MT dynamics due to the oscillations in cytosolic $\left[\mathrm{Ca}^{2+}\right]$ is expected. As mentioned before the tubulin polymerisation is inhibited in the presence of $\mathrm{Ca}^{2+}$ in vitro. However, the protein make-up of MT ends defines the net action of $\mathrm{Ca}^{2+}$. Interestingly, in the presence of both MAPs and $\mathrm{Ca}^{2+}$, MTs are longer and more curved in vitro, which is explained by the possibility of $\mathrm{Ca}^{2+}$ binding to several different classes of binding sites in the MT-MAPs system (V. Buljan et al., 2009). Moreover, using fluorescently-labelled tubulin, it has been demonstrated that the mitotic spindle structure in most of the $\mathrm{Ca}^{2+}$ chelator-treated cells gradually deforms with time (Xu et al., 2003).

In order to determine whether the effects of Kv10.1 downregulation on MT dynamics are explicitly due to its conductance, experiments involving specific blockers of the channel were performed. The antihistamine drug astemizole efficiently blocks Kv10.1 current (García-Ferreiro et al., 2004a). Contrary to our expectations, blocking the channel conductance with astemizole did not show any effect when compared to DMSO treated cells. At the same time, its isomer norastemizole, which retains antihistamine activity but has no channel inhibitory activity (García-Ferreiro et al., 2004b), showed increased MT dynamics. However, both compounds are implicated in the regulation of cytosolic calcium (Fischer et al., 1997), making the interpretation of the observed results ambiguous. Nevertheless, comparing norastemizole and astemizole conditions only and considering the specificity of the drugs for Kv10.1 


\section{DisCUSSION}

inhibition is the only difference between the two, we can conclude that possibly Kv10.1 current inhibition decreases MT dynamicity (Figure 3.14). To discard the possible effects of the drugs unrelated to their action on Kv10.1, we used a blocking monoclonal antibody (mAb56) directed against the pore loop of Kv10.1 (GomezVarela et al., 2007). Like in the case of norastemizole/astemizole inhibition of Kv10.1 currents with mAb56 resulted in decreased MT dynamics (Figure 3.15). Moreover, inhibition of ORAI1 mimicked the effect of mAb56 on the MT dynamics: decrease in growth rate, growth length and dynamicity (Figure 3.16).

On a first look, it may seem that these results are contradictory with our initial observation that silencing of Kv10.1 increases MT dynamics. However, as already mentioned, Kv10.1 is characterised by large cytosolic domains and localises to the centrosomes, as the vast majority of the cell cycle regulators. This creates a unique opportunity for Kv10.1 to interact or may even serve as a scaffold for the initiation of a signalling cascade. An attractive option will be interaction with PLK1 or Aurora A. Additionally, one of the splice forms of Kv10.1 (E65) triggers CDK1 activation (Ramos Gomes et al., 2015). Therefore, our data point to a dual effect of Kv10.1 on MT dynamics: on one hand, through a mechanism unrelated to permeation, it promotes the dynamicity of MTs, while on the other hand, its function as a channel promotes stability. Therefore, when inhibiting the conductance of the channel, we are capable of dissecting these opposing effects, whereas silencing diminishes both. 


\section{Chapter 5}

\section{Conclusion \& Perspectives}

We show here that downregulation of Kv10.1 modulates the behaviour of MT during mitosis, leading to activation of SAC and delay in metaphase-to-anaphase transition. The extended metaphase and enhanced SAC prevent the formation of lagging chromosomes, commonly observed in cancer cells.

The described phenotype is achieved partially by functionally interacting with ORAI1 and changing the cytosolic $\mathrm{Ca}^{2+}$ behaviour, both in terms of global concentration and in the properties of calcium oscillations. As a result, downstream targets such as Aurora A, PLK1 and MT dynamics are altered, and SAC is activated. Kv10.1 also influences MT dynamics independently of its conductance, suggesting that it possesses a dual effect: On one side, predominantly through its canonical function as an ion channel, it promotes the dynamicity of MTs; on the other hand, probably through its intracellular domains and splice variants, it promotes MT stability. By inhibiting the conductive function of the channel, we were capable of dissecting these opposing effects.

Future experiments should focus on better understanding the functional interaction between Kv10.1 and ORAI1 and the function of the complex in the regulation of calcium oscillations, and on the identification of the decoders of the signal, likely Aurora A or PLK1. As a priority for future experiments, we should mention the study of calcium dynamics upon inhibition of Kv10.1 using a mAb56 monoclonal antibody, that should be studied with higher temporal resolution. An already known mutant form of the channel whose conductance has been abolished, together with the short splice variants should also help to dissect the dual effect that Kv10.1 exerts on MTs.

In general, understanding of how Kv10.1 modulates the MT architecture within a tissue, especially when positioning the mitotic spindle, will help to find out whether Kv10.1 is also essential for cell differentiation or EMT and, globally, how a cancer cell benefits from the presence of Kv10.1. 
Conclusion \& Perspectives 


\section{References}

Abal, M., Andreu, J. M., \& Barasoain, I. (2003, jun). Taxanes: microtubule and centrosome targets, and cell cycle dependent mechanisms of action. Curr. Cancer Drug Targets, 3(3), 193-203. Retrieved from http://www.ncbi.nlm .nih.gov/pubmed/12769688

Adaixo, R., Harley, C. A., Castro-Rodrigues, A. F., \& Morais-Cabral, J. H. (2013, mar). Structural properties of PAS domains from the $\mathrm{KCNH}$ potassium channels. PLoS One, 8(3), e59265. Retrieved from https://dx.plos.org/ 10.1371/journal.pone.0059265 doi: 10.1371/journal.pone.0059265

Adams, P. D., \& Kaelin, W. G. (1995, apr). Transcriptional control by E2F. Semin. Cancer Biol., 6(2), 99-108. Retrieved from http://linkinghub.elsevier .com/retrieve/pii/S1044579X85700132 doi: 10.1006/scbi.1995.0013

Agarwal, J. R., Griesinger, F., Stühmer, W., \& Pardo, L. A. (2010, jan). The potassium channel Ether à go-go is a novel prognostic factor with functional relevance in acute myeloid leukemia. Mol. Cancer, 9(1), 18. Retrieved from http://molecular-cancer.biomedcentral.com/articles/ 10.1186/1476-4598-9-18 doi: 10.1186/1476-4598-9-18

Agircan, F. G., Schiebel, E., \& Mardin, B. R. (2014). Separate to operate: control of centrosome positioning and separation. Philos. Trans. R. Soc. Lond. B. Biol. Sci., 369(1650), 20130461-20130461. Retrieved from http://rstb .royalsocietypublishing.org/cgi/doi/10.1098/rstb.2013.0461 doi: $10.1098 /$ rstb.2013.0461

Aiello, N. M., Maddipati, R., Norgard, R. J., Balli, D., Li, J., Yuan, S., ... Stanger, B. Z. (2018, jun). EMT Subtype Influences Epithelial Plasticity and Mode of Cell Migration. Dev. Cell, 45(6), 681-695.e4. Retrieved from https:// WWW.sciencedirect.com/science/article/pii/S1534580718304209 doi: 10.1016/J.DEVCEL.2018.05.027

Akhmanova, A., \& Steinmetz, M. O. (2015, nov). Control of microtubule organization and dynamics: two ends in the limelight. Nat. Rev. Mol. Cell Biol., 16, 711. Retrieved from https://doi.org/10.1038/nrm4084 


\section{REFERENCES}

Amin, M. A., Itoh, G., Iemura, K., Ikeda, M., \& Tanaka, K. (2014, jul). CLIP-170 recruits PLK1 to kinetochores during early mitosis for chromosome alignment. J. Cell Sci., 127(13), 2818-2824. Retrieved from http://www.ncbi.nlm.nih .gov/pubmed/24777477 doi: $10.1242 /$ jcs. 150755

Applegate, K. T., Besson, S., Matov, A., Bagonis, M. H., Jaqaman, K., \& Danuser, G. (2011, nov). plusTipTracker: Quantitative image analysis software for the measurement of microtubule dynamics. J. Struct. Biol., 176(2), 168184. Retrieved from http://www.ncbi.nlm.nih.gov/pubmed/21821130 doi: 10.1016/j.jsb.2011.07.009

Applegate, K. T., \& Danuser, G. (2010). Technical Report: Using the plusTip Tracker Software to Measure and Analyze Microtubule Dynamics from + TIP Comets (Tech. Rep.).

Arcangeli, A., Becchetti, A., Cherubini, A., Crociani, O., Defilippi, P., Guasti, L., ... Wanke, E. (2004, nov). Physical and functional interaction between integrins and hERG potassium channels. Biochem. Soc. Trans., 32(Pt 5), 8267. Retrieved from http://www.ncbi.nlm.nih.gov/pubmed/15494025 doi: 10.1042/BST0320826

Asher, V., Khan, R., Warren, A., Shaw, R., Schalkwyk, G. V., Bali, A., \& Sowter, H. M. (2010, dec). The Eag potassium channel as a new prognostic marker in ovarian cancer. Diagn. Pathol., 5(1), 78. Retrieved from https://diagnosticpathology.biomedcentral.com/ articles/10.1186/1746-1596-5-78 doi: 10.1186/1746-1596-5-78

Ashkenazi, R., Gentry, S. N., \& Jackson, T. L. (2008, nov). Pathways to tumorigenesismodeling mutation acquisition in stem cells and their progeny. Neoplasia, 10(11), 1170-82. Retrieved from http://www.ncbi.nlm.nih.gov/pubmed/ 18953426

Asteriti, I. A., De Mattia, F., \& Guarguaglini, G. (2015). Cross-Talk between AURKA and Plk1 in Mitotic Entry and Spindle Assembly. Front. Oncol., 5, 283. Retrieved from http://www.ncbi.nlm.nih.gov/pubmed/26779436 doi: 10.3389/fonc. 2015.00283

Awasthi, P., Foiani, M., \& Kumar, A. (2015, dec). ATM and ATR signaling at a glance. J. Cell Sci., 128(23), 4255 LP - 4262. Retrieved from http://jcs.biologists 
.org/content/128/23/4255. abstract doi: 10.1242/jcs.169730

Azqueta, A., \& Collins, A. R. (2014). The Comet Assay: High Throughput Use of FPG. In (pp. 199-217). Humana Press, New York, NY. Retrieved from http://Iink.springer.com/10.1007/978-1-4939-1068-7\{_\}12 doi: 10.1007/978-1-4939-1068-7_12

Badaoui, M., Mimsy-Julienne, C., Saby, C., Van Gulick, L., Peretti, M., Jeannesson, P., ... Ouadid-Ahidouch, H. (2018, may). Collagen type 1 promotes survival of human breast cancer cells by overexpressing Kv10.1 potassium and Orai1 calcium channels through DDR1-dependent pathway. Oncotarget, 9(37), 2465324671. Retrieved from http://www.ncbi.nlm.nih.gov/pubmed/29872495 doi: 10.18632/oncotarget.19065

Bai, Y., Liao, H., Liu, T., Zeng, X., Xiao, F., Luo, L., .. Guo, L. (2013, feb). MiR-296-3p regulates cell growth and multi-drug resistance of human glioblastoma by targeting ether-à-go-go (EAG1). Eur. J. Cancer, 49(3), 710-24. Retrieved from https://linkinghub.elsevier.com/retrieve/pii/ S095980491200682X doi: 10.1016/j.ejca.2012.08.020

Barnes, L. D., Engel, A. G., \& Dousa, T. P. (1975). Studies on in vitro polymerization of tubulin from renal medullary extracts. Biochim. Biophys. Acta-Protein Struct., 405(2), 422-433. Retrieved from http://www.sciencedirect.com/ science/article/pii/0005279575901075 doi: https://doi.org/10.1016/ 0005-2795(75)90107-5

Barros, F., Domínguez, P., \& de la Peña, P. (2012). Cytoplasmic domains and voltage-dependent potassium channel gating. Front. Pharmacol., 3, 49. Retrieved from http://journal.frontiersin.org/article/10.3389/ fphar.2012.00049/abstract doi: 10.3389/fphar.2012.00049

Bartek, J., \& Lukas, J. (2003, may). Chk1 and Chk2 kinases in checkpoint control and cancer. Cancer Cell, 3(5), 421-9. Retrieved from http://www.ncbi.nlm .nih.gov/pubmed/12781359

Bastians, H. (2015). Causes of Chromosomal Instability. In Recent results cancer res. (Vol. 200, pp. 95-113). Retrieved from http://www.ncbi.nlm.nih.gov/ pubmed/26376874 doi: 10.1007/978-3-319-20291-4_5

Baumann, K. (2014, aug). Nuclear envelope: ATR senses mechanical stress. Nat. 


\section{REFERENCES}

Rev. Mol. Cell Biol., 15(9), 559-559. Retrieved from http://www.nature.com/ doifinder/10.1038/nrm3864 doi: 10.1038/nrm3864

Becchetti, A., \& Arcangeli, A. (2010). Integrins and ion channels in cell migration: implications for neuronal development, wound healing and metastatic spread. Adv. Exp. Med. Biol., 674, 107-23. Retrieved from http://www.ncbi.nlm .nih.gov/pubmed/20549944

Benito, J., Shi, Y., Szymanska, B., Carol, H., Boehm, I., Lu, H., .. Konopleva, M. (2011, aug). Pronounced hypoxia in models of murine and human leukemia: high efficacy of hypoxia-activated prodrug PR-104. PLoS One, 6(8), e23108. Retrieved from http://dx.plos.org/10.1371/journal.pone.0023108 doi: 10.1371/journal.pone.0023108

Bergstralh, D. T., \& St Johnston, D. (2014, oct). Spindle orientation: What if it goes wrong? Semin. Cell Dev. Biol., 34, 140-145. Retrieved from https:// WWW.sciencedirect.com/science/article/pii/S1084952114001876 doi: 10.1016/J.SEMCDB.2014.06.014

Berkowitz, S. A., \& Wolff, J. (1981, nov). Intrinsic calcium sensitivity of tubulin polymerization. The contributions of temperature, tubulin concentration, and associated proteins. J. Biol. Chem., 256(21), 11216-23. Retrieved from http://www.ncbi.nlm.nih.gov/pubmed/7287764

Bezanilla, F. (2008, apr). How membrane proteins sense voltage. Nat. Rev. Mol. Cell Biol., 9(4), 323-332. Retrieved from http://www.ncbi.nlm.nih.gov/ pubmed/18354422 doi: 10.1038/nrm2376

Bijlenga, P., Occhiodoro, T., Liu, J. H., Bader, C. R., Bernheim, L., \& FischerLougheed, J. (1998, oct). An ether -à-go-go K+ current, Ih-eag, contributes to the hyperpolarization of human fusion-competent myoblasts. J. Physiol., 512 ( Pt 2, 317-23. Retrieved from http://www.ncbi.nlm.nih.gov/pubmed/ 9763622

Bodnar, A. G., Ouellette, M., Frolkis, M., Holt, S. E., Chiu, C. P., Morin, G. B., ... Wright, W. E. (1998, jan). Extension of life-span by introduction of telomerase into normal human cells. Science, 279(5349), 349-52. Retrieved from http://www.ncbi.nlm.nih.gov/pubmed/9454332

Bortner, C. D., \& Cidlowski, J. A. (2007, jun). Cell shrinkage and monovalent cation 
fluxes: Role in apoptosis. Arch. Biochem. Biophys., 462(2), 176-188. Retrieved from http://www.ncbi.nlm.nih.gov/pubmed/17321483 doi: 10.1016/j.abb .2007 .01 .020

Bortner, C. D., \& Cidlowski, J. A. (2014, feb). Ion channels and apoptosis in cancer. Philos. Trans. R. Soc. B Biol. Sci., 369 (1638), 20130104-20130104. Retrieved from http://www.ncbi.nlm.nih.gov/pubmed/24493752 doi: 10.1098/rstb .2013 .0104

Bowey-Dellinger, K., Dixon, L., Ackerman, K., Vigueira, C., Suh, Y. K., Lyda, T., ... Segarra, V. A. (2017). Introducing Mammalian Cell Culture and Cell Viability Techniques in the Undergraduate Biology Laboratory. J. Microbiol. Biol. Educ., 18(2). Retrieved from http://www.ncbi.nlm.nih.gov/pubmed/ 28861134 doi: 10.1128/jmbe.v18i2.1264

Brouhard, G. J., Stear, J. H., Noetzel, T. L., Al-Bassam, J., Kinoshita, K., Harrison, S. C., ... Hyman, A. A. (2008, jan). XMAP215 Is a Processive Microtubule Polymerase. Cell, 132(1), 79-88. Retrieved from http://www.ncbi.nlm.nih .gov/pubmed/18191222 doi: 10.1016/j.cell.2007.11.043

Brüggemann, A., Pardo, L. A., Stühmer, W., \& Pongs, O. (1993, sep). Ether-à-go-go encodes a voltage-gated channel permeable to $\mathrm{K}+$ and $\mathrm{Ca} 2+$ and modulated by cAMP. Nature, 365(6445), 445-8. Retrieved from http://www.nature.com/ articles/365445a0 doi: 10.1038/365445a0

Brüggemann, A., Stühmer, W., \& Pardo, L. A. (1997, jan). Mitosis-promoting factor-mediated suppression of a cloned delayed rectifier potassium channel expressed in Xenopus oocytes. Proc. Natl. Acad. Sci. U. S. A., 94(2), 537-42. Retrieved from http://www.ncbi.nlm.nih.gov/pubmed/9012819

Bruinsma, W., Aprelia, M., García-Santisteban, I., Kool, J., Xu, Y. J., \& Medema, R. H. (2017, mar). Inhibition of Polo-like kinase 1 during the DNA damage response is mediated through loss of Aurora A recruitment by Bora. Oncogene, 36(13), 1840-1848. Retrieved from https://www.ncbi.nlm.nih.gov/pubmed/ 27721411 doi: 10.1038/onc.2016.347

Bruinsma, W., Raaijmakers, J. A., \& Medema, R. H. (2012, dec). Switching Polo-like kinase-1 on and off in time and space. Trends Biochem. Sci., 37(12), 534542. Retrieved from http://www.ncbi.nlm.nih.gov/pubmed/23141205 doi: 


\section{REFERENCES}

10.1016/j.tibs.2012.09.005

Buljan, V., Ivanova, E. P., \& Cullen, K. M. (2009, apr). How calcium controls microtubule anisotropic phase formation in the presence of microtubuleassociated proteins in vitro. Biochem. Biophys. Res. Commun., 381(2), 224228. Retrieved from http://www.ncbi.nlm.nih.gov/pubmed/19338777 doi: 10.1016/j.bbrc.2009.02.028

Buljan, V. A., Graeber, M. B., Holsinger, R. M. D., Brown, D., Hambly, B. D., Delikatny, E. J., ... Banati, R. B. (2018, mar). Calcium-axonemal microtubuli interactions underlie mechanism(s) of primary cilia morphological changes. $J$. Biol. Phys., 44(1), 53-80. Retrieved from http://www.ncbi.nlm.nih.gov/ pubmed/29090363 doi: 10.1007/s10867-017-9475-2

Burbank, K. S., \& Mitchison, T. J. (2006, jul). Microtubule dynamic instability. Curr. Biol., 16(14), R516-7. Retrieved from http://www.ncbi.nlm.nih.gov/ pubmed/16860721 doi: 10.1016/j.cub.2006.06.044

Burgess, R. C., Burman, B., Kruhlak, M. J., \& Misteli, T. (2014, dec). Activation of DNA Damage Response Signaling by Condensed Chromatin. Cell Rep., 9(5), 1703-1717. Retrieved from http://www.ncbi.nlm.nih.gov/pubmed/ 25464843 doi: 10.1016/j.celrep.2014.10.060

Burma, S., Chen, B. P., Murphy, M., Kurimasa, A., \& Chen, D. J. (2001, nov). ATM Phosphorylates Histone H2AX in Response to DNA Double-strand Breaks. J. Biol. Chem., 276(45), 42462-42467. Retrieved from http:// www.ncbi.nlm.nih.gov/pubmed/11571274 doi: 10.1074/jbc.C100466200

Cai, W., \& Yang, H. (2016). The structure and regulation of Cullin 2 based E3 ubiquitin ligases and their biological functions. Cell Div., 11, 7. Retrieved from http://www.ncbi.nlm.nih.gov/pubmed/27222660 doi: 10.1186/s13008-016 $-0020-7$

Cain, K., Langlais, C., Sun, X. M., Brown, D. G., \& Cohen, G. M. (2001, nov). Physiological concentrations of $\mathrm{K}+$ inhibit cytochrome c-dependent formation of the apoptosome. J. Biol. Chem., 276(45), 41985-90. Retrieved from http://www.jbc.org/lookup/doi/10.1074/jbc.M107419200 doi: 10.1074/ jbc.M107419200

Camacho, J., Sánchez, A., Stühmer, W., \& Pardo, L. A. (2000, dec). Cy- 
toskeletal interactions determine the electrophysiological properties of human EAG potassium channels. Pflugers Arch., 441(2-3), 167-74. Retrieved from http://www.ncbi.nlm.nih.gov/pubmed/11211100

Carrassa, L., Sanchez, Y., Erba, E., \& Damia, G. (2009, aug). U2OS cells lacking Chk1 undergo aberrant mitosis and fail to activate the spindle checkpoint. J. Cell. Mol. Med., 13(8a), 1565-1576. Retrieved from http://www.ncbi.nlm.nih.gov/ pubmed/19778378 doi: 10.1111/j.1582-4934.2009.00362.x

Catsch, A. (1948, mar). Eine Erbliche Störung Des Bewegungsmechanismus Bei Drosophila Melanogaster. Z. Indukt. Abstamm. Vererbungsl., 82(1), 6466. Retrieved from https://doi.org/10.1007/BF00308399 doi: 10.1007/ BF00308399

Chabalier-Taste, C., Racca, C., Dozier, C., \& Larminat, F. (2008, dec). BRCA1 is regulated by Chk2 in response to spindle damage. Biochim. Biophys. ActaMol. Cell Res., 1783(12), 2223-2233. Retrieved from http://www.ncbi.nlm .nih.gov/pubmed/18804494 doi: 10.1016/j.bbamcr.2008.08.006

Chal, J., \& Pourquié, O. (2017, jun). Making muscle: skeletal myogenesis <i $>$ in vivo $</ \mathrm{i}>$ and $<\mathrm{i}>$ in vitro $</ \mathrm{i}>$. Development, $144(12), 2104-2122$. Retrieved from http://www.ncbi.nlm.nih.gov/pubmed/28634270 doi: 10.1242/dev .151035

Charnley, M., Anderegg, F., Holtackers, R., Textor, M., \& Meraldi, P. (2013, jun). Effect of Cell Shape and Dimensionality on Spindle Orientation and Mitotic Timing. PLoS One, 8(6), e66918. Retrieved from http://www.ncbi.nlm.nih .gov/pubmed/23825020 doi: 10.1371/journal.pone.0066918

Cheeseman, L. P., Harry, E. F., McAinsh, A. D., Prior, I. A., \& Royle, S. J. (2013, may). Specific removal of TACC3-ch-TOG-clathrin at metaphase deregulates kinetochore fiber tension. J. Cell Sci., 126(9), 2102-2113. Retrieved from http://www.ncbi.nlm.nih.gov/pubmed/23532825 doi: 10.1242/jcs.124834

Chen, Y., \& Poon, R. Y. C. (2008, may). The multiple checkpoint functions of CHK1 and CHK2 in maintenance of genome stability. Front. Biosci., 13, 5016-29. Retrieved from http://www.ncbi.nlm.nih.gov/pubmed/18508566

Chen, Y., Sánchez, A., Rubio, M. E., Kohl, T., Pardo, L. A., \& Stühmer, W. (2011, may). Functional K(v)10.1 channels localize to the inner nuclear membrane. 


\section{REFERENCES}

PLoS One, 6(5), e19257. Retrieved from https://dx.plos.org/10.1371/ journal.pone.0019257 doi: 10.1371/journal.pone.0019257

Cherubini, A., Hofmann, G., Pillozzi, S., Guasti, L., Crociani, O., Cilia, E., ... Arcangeli, A. (2005, jun). Human $<$ i $>$ ether-a-go-go $</ \mathrm{i}>$-related Gene 1 Channels Are Physically Linked to $\beta<$ sub $>1</$ sub $>$ Integrins and Modulate Adhesion-dependent Signaling. Mol. Biol. Cell, 16(6), 2972-2983. Retrieved from http://www.ncbi.nlm.nih.gov/pubmed/15800067 doi: 10.1091/mbc .e04-10-0940

Cherubini, A., Pillozzi, S., Hofmann, G., Crociani, O., Guasti, L., Lastraioli, E., ... Arcangeli, A. (2002, nov). HERG K+ channels and beta1 integrins interact through the assembly of a macromolecular complex. Ann. N. Y. Acad. Sci., 973, 559-61. Retrieved from http://www.ncbi.nlm.nih.gov/pubmed/12485929

Chow, J. P. H., Poon, R. Y. C., \& Ma, H. T. (2011, apr). Inhibitory Phosphorylation of Cyclin-Dependent Kinase 1 as a Compensatory Mechanism for Mitosis Exit. Mol. Cell. Biol., 31(7), 1478-1491. Retrieved from http://www.ncbi.nlm.nih .gov/pubmed/21262764 doi: 10.1128/MCB.00891-10

Cipressa, F., \& Cenci, G. (2013). DNA damage response, checkpoint activation and dysfunctional telomeres: face to face between mammalian cells and Drosophila. Tsitologiia, 55(4), 211-7. Retrieved from http://www.ncbi.nlm.nih.gov/ pubmed/23875450

Cirillo, L., Gotta, M., \& Meraldi, P. (2017). The Elephant in the Room: The Role of Microtubules in Cancer. In Adv. exp. med. biol. (Vol. 1002, pp. 93124). Retrieved from http://www.ncbi.nlm.nih.gov/pubmed/28600784 doi: 10.1007/978-3-319-57127-0_5

Cleveland, D. W., Mao, Y., \& Sullivan, K. F. (2003, feb). Centromeres and Kinetochores: From Epigenetics to Mitotic Checkpoint Signaling. Cell, 112(4), 407-421. Retrieved from https://doi.org/10.1016/S0092-8674(03)00115 -6 doi: 10.1016/S0092-8674(03)00115-6

Collins, T. J. (2007, jul). ImageJ for microscopy. Biotechniques, 43(1S), S25S30. Retrieved from http://www.ncbi.nlm.nih.gov/pubmed/17936939 doi: $10.2144 / 000112517$

Cooper, E. (2001, may). A new role for ion channels in myoblast fusion. $J$. 
Cell Biol., 153(4), F9-12. Retrieved from http://www.ncbi.nlm.nih.gov/ pubmed/11352947

Cooper, G. M. (2000). The Cell Cycle. Retrieved from https://www.ncbi.nlm.nih .gov/books/NBK9852/

Cory, J. G., \& Sato, A. (1983). Regulation of ribonucleotide reductase activity in mammalian cells. Mol. Cell. Biochem., 53-54(1-2), 257-66. Retrieved from http://www.ncbi.nlm.nih.gov/pubmed/6353195

Coverley, D., Laman, H., \& Laskey, R. A. (2002, jul). Distinct roles for cyclins E and A during DNA replication complex assembly and activation. Nat. Cell Biol., 4(7), 523-8. Retrieved from http://www. nature.com/articles/ncb813 doi: $10.1038 /$ ncb813

Dai, G., Qian, Y., Chen, J., Meng, F.-L., Pan, F.-Y., Shen, W.-G., ... Li, C.-J. (2013, oct). Calmodulin activation of polo-like kinase 1 is required during mitotic entry. Biochem. Cell Biol., 91(5), 287-94. Retrieved from http://www .nrcresearchpress.com/doi/10.1139/bcb-2013-0015 doi: 10.1139/bcb $-2013-0015$

Dalby, B., Cates, S., Harris, A., Ohki, E. C., Tilkins, M. L., Price, P. J., \& Ciccarone, V. C. (2004, jun). Advanced transfection with Lipofectamine 2000 reagent: primary neurons, siRNA, and high-throughput applications. Methods, 33(2), 95-103. Retrieved from http://www.ncbi.nlm.nih.gov/pubmed/15121163 doi: 10.1016/j.ymeth.2003.11.023

D’Avino, P. P., Giansanti, M. G., \& Petronczki, M. (2015, apr). Cytokinesis in Animal Cells. Cold Spring Harb. Perspect. Biol., 7(4), a015834. Retrieved from http://www.ncbi.nlm.nih.gov/pubmed/25680833 doi: 10.1101/cshperspect .a015834

Dekker, J. (2014, dec). Two ways to fold the genome during the cell cycle: insights obtained with chromosome conformation capture. Epigenetics Chromatin, 7(1), 25. Retrieved from https://epigeneticsandchromatin.biomedcentral .com/articles/10.1186/1756-8935-7-25 doi: 10.1186/1756-8935-7-25

de la Pompa, J. L. (1994, jul). Functional relationships between genes of the Shaker gene complex of Drosophila. Mol. Gen. Genet., 244(2), 197-204. Retrieved from http://www.ncbi.nlm.nih.gov/pubmed/8052239 


\section{REFERENCES}

DeLoughery, Z., Luczak, M. W., Ortega-Atienza, S., \& Zhitkovich, A. (2015, jan). DNA Double-Strand Breaks by Cr(VI) Are Targeted to Euchromatin and Cause ATR-Dependent Phosphorylation of Histone H2AX and Its Ubiquitination. Toxicol. Sci., 143(1), 54-63. Retrieved from http://www.ncbi.nlm.nih.gov/ pubmed/25288669 doi: 10.1093/toxsci/kfu207

Deng, H., Luo, T., Vorperian, S., Petzold, C., \& Nelson, D. (2014). Dopamine Reuptake Inhibition as the Means of Antidepressant Mechanism of Function (LB92). FASEB J., 28(1_supplement), LB92. Retrieved from https://www .fasebj.org/doi/abs/10.1096/fasebj.28.1\{_\}supplement.1b92 doi: 10 .1096/fasebj.28.1_supplement.lb92

Diaz, L., Ceja-Ochoa, I., Restrepo-Angulo, I., Larrea, F., Avila-Chavez, E., GarciaBecerra, R., .. Camacho, J. (2009, apr). Estrogens and Human Papilloma Virus Oncogenes Regulate Human Ether-a-go-go-1 Potassium Channel Expression. Cancer Res., 69(8), 3300-3307. Retrieved from http://www.ncbi.nlm .nih.gov/pubmed/19351862 doi: 10.1158/0008-5472.CAN-08-2036

Ding, X.-W., Luo, H.-s., Jin, X., Yan, J.-j., \& Ai, Y.-w. (2007). Aberrant expression of Eag1 potassium channels in gastric cancer patients and cell lines. Med. Oncol., $24(3), 345-50$. Retrieved from http://www.ncbi.nlm.nih.gov/pubmed/ 17873312

Ding, X.-W., Wang, X.-G., Luo, H.-S., Tan, S.-Y., Gao, S., Luo, B., \& Jiang, H. (2008, aug). Expression and prognostic roles of Eag1 in resected esophageal squamous cell carcinomas. Dig. Dis. Sci., 53(8), 2039-44. Retrieved from http://link.springer.com/10.1007/s10620-007-0116-7 doi: 10.1007/ s10620-007-0116-7

Donzelli, M., \& Draetta, G. F. (2003, jul). Regulating mammalian checkpoints through Cdc25 inactivation. EMBO Rep., 4(7), 671-677. Retrieved from http://www.ncbi.nlm.nih.gov/pubmed/12835754 doi: 10.1038/sj.embor .embor887

Dowdy, S. F., Hinds, P. W., Louie, K., Reed, S. I., Arnold, A., \& Weinberg, R. A. (1993, may). Physical interaction of the retinoblastoma protein with human D cyclins. Cell, $73(3), 499-511$. Retrieved from http://www.ncbi.nlm.nih.gov/ pubmed/8490963 
Downie, B. R., Sanchez, A., Knotgen, H., Contreras-Jurado, C., Gymnopoulos, M., Weber, C., ... Pardo, L. A. (2008, dec). Eag1 Expression Interferes with Hypoxia Homeostasis and Induces Angiogenesis in Tumors. J. Biol. Chem., 283(52), 36234-36240. Retrieved from http://www.jbc.org/lookup/doi/ 10.1074/jbc.M801830200 doi: 10.1074/jbc.M801830200

Dreixler, J. C., Bian, J., Cao, Y., Roberts, M. T., Roizen, J. D., \& Houamed, K. M. (2000, jul). Block of rat brain recombinant SK channels by tricyclic antidepressants and related compounds. Eur. J. Pharmacol., 401(1), 1-7. Retrieved from http://www.ncbi.nlm.nih.gov/pubmed/10915830

Eliezer, Y., Argaman, L., Kornowski, M., Roniger, M., \& Goldberg, M. (2014, mar). Interplay between the DNA damage proteins MDC1 and ATM in the regulation of the spindle assembly checkpoint. J. Biol. Chem., 289(12), 81828193. Retrieved from http://www.ncbi.nlm.nih.gov/pubmed/24509855 doi: 10.1074/jbc.M113.532739

Enders, G. H. (2008, jun). Expanded roles for Chk1 in genome maintenance. J. Biol. Chem., 283(26), 17749-52. Retrieved from http://www.ncbi.nlm.nih.gov/ pubmed/18424430 doi: 10.1074/jbc.R800021200

Erneux, C., Passareiro, H., \& Nunez, J. (1984, jul). Interaction between calmodulin and microtubule-associated proteins prepared at different stages of brain development. FEBS Lett., 172(2), 315-20. Retrieved from http:// www.ncbi.nlm.nih.gov/pubmed/6430720

Ertych, N., Stolz, A., Stenzinger, A., Weichert, W., Kaulfuß, S., Burfeind, P., ... Bastians, H. (2014, jun). Increased microtubule assembly rates influence chromosomal instability in colorectal cancer cells. Nat. Cell Biol., 16(8), 779791. Retrieved from http://www.ncbi.nlm.nih.gov/pubmed/24976383 doi: $10.1038 /$ ncb2994

Ertych, N., Stolz, A., Valerius, O., Braus, G. H., \& Bastians, H. (2016, feb). $<\mathrm{i}>\mathrm{CHK} 2</ \mathrm{i}>-<\mathrm{i}>\mathrm{BRCA} 1</ \mathrm{i}>$ tumor-suppressor axis restrains oncogenic Aurora-A kinase to ensure proper mitotic microtubule assembly. Proc. Natl. Acad. Sci., 113(7), 1817-1822. Retrieved from http://www.ncbi.nlm.nih .gov/pubmed/26831064 doi: 10.1073/pnas.1525129113

Ferguson, R. L., \& Maller, J. L. (2010, may). Centrosomal Localization of Cyclin 


\section{REFERENCES}

E-Cdk2 Is Required for Initiation of DNA Synthesis. Curr. Biol., 20(9), 856860. Retrieved from https://www.sciencedirect.com/science/article/ pii/S0960982210003532 doi: 10.1016/J.CUB.2010.03.028

Ferrara, N., \& Davis-Smyth, T. (1997, feb). The Biology of Vascular Endothelial Growth Factor. Endocr. Rev., 18(1), 4-25. Retrieved from http://www.ncbi .nlm.nih.gov/pubmed/9034784 doi: 10.1210/edrv.18.1.0287

Fischer, M. J., Paulussen, J. J., Kok-Van Esterik, J. A., Van der Heijden, V. S., De Mol, N. J., \& Janssen, L. H. (1997, mar). Effects of the anti-allergics astemizole and norastemizole on Fc epsilon RI receptor-mediated signal transduction processes. Eur. J. Pharmacol., 322(1), 97-105. Retrieved from http:// wWw.ncbi.nlm.nih.gov/pubmed/9088877

Florian, S., \& Mitchison, T. J. (2016). Anti-Microtubule Drugs. In Methods mol. biol. (Vol. 1413, pp. 403-421). Retrieved from http://www.ncbi.nlm.nih.gov/ pubmed/27193863 doi: 10.1007/978-1-4939-3542-0_25

Fourest-Lieuvin, A., Peris, L., Gache, V., Garcia-Saez, I., Juillan-Binard, C., Lantez, V., \& Job, D. (2006, mar). Microtubule regulation in mitosis: tubulin phosphorylation by the cyclin-dependent kinase Cdk1. Mol. Biol. Cell, 17(3), 1041-50. Retrieved from http://www.ncbi.nlm.nih.gov/pubmed/16371510 doi: 10.1091/mbc.e05-07-0621

Fourier, J.-B.-J. (1822). Th[́e Jorie analytique de la chaleur. Paris: F. Didot. Retrieved from https://www.worldcat.org/title/theorie-analytique-de -la-chaleur/oclc/2688081

Fraschini, R. (2016). Factors that Control Mitotic Spindle Dynamics. In (pp. 89-101). Springer, Singapore. Retrieved from http://link.springer.com/10.1007/ 5584\{_\}2016\{_\}74 doi: 10.1007/5584_2016_74

Fredriksson, S., Gullberg, M., Jarvius, J., Olsson, C., Pietras, K., Gústafsdóttir, S. M., ... Landegren, U. (2002, may). Protein detection using proximity-dependent DNA ligation assays. Nat. Biotechnol., 20(5), 473-7. Retrieved from http:// www.nature.com/articles/nbt0502-473 doi: 10.1038/nbt0502-473

Friedman, P. A., \& Platzer, E. G. (1978, dec). Interaction of anthelmintic benzimidazoles and benzimidazole derivatives with bovine brain tubulin. Biochim. Biophys. Acta, 544(3), 605-14. Retrieved from http://www.ncbi.nlm.nih.gov/ 
pubmed/728472

Galjart, N. (2010, jun). Plus-End-Tracking Proteins and Their Interactions at Microtubule Ends. Curr. Biol., 20(12), R528-R537. Retrieved from http:// www.ncbi.nlm.nih.gov/pubmed/20620909 doi: 10.1016/j.cub.2010.05.022

García-Ferreiro, R. E., Kerschensteiner, D., Major, F., Monje, F., Stühmer, W., \& Pardo, L. A. (2004a, oct). Mechanism of block of hEag1 K+ channels by imipramine and astemizole. J. Gen. Physiol., 124(4), 301-17. Retrieved from http://www.ncbi.nlm.nih.gov/pubmed/15365094 doi: 10.1085/jgp .200409041

García-Ferreiro, R. E., Kerschensteiner, D., Major, F., Monje, F., Stühmer, W., \& Pardo, L. A. (2004b, oct). Mechanism of Block of hEag1 $\mathrm{K}<\sup >+</ \sup >$ Channels by Imipramine and Astemizole. J. Gen. Physiol., 124(4), 301317. Retrieved from http://www.ncbi.nlm.nih.gov/pubmed/15365094 doi: 10.1085/jgp.200409041

Gardner, M. K., Zanic, M., \& Howard, J. (2013, feb). Microtubule catastrophe and rescue. Curr. Opin. Cell Biol., 25(1), 14-22. Retrieved from https:// www.ncbi.nlm.nih.gov/pubmed/23092753 doi: 10.1016/j.ceb.2012.09.006

Gately, D. P., Hittle, J. C., Chan, G. K., \& Yen, T. J. (1998, sep). Characterization of ATM expression, localization, and associated DNA-dependent protein kinase activity. Mol. Biol. Cell, 9(9), 2361-74. Retrieved from http://www.ncbi .nlm.nih.gov/pubmed/9725899

Gavrilova-Ruch, O., Schönherr, K., Gessner, G., Schönherr, R., Klapperstück, T., Wohlrab, W., \& Heinemann, S. H. (2002, jul). Effects of imipramine on ion channels and proliferation of IGR1 melanoma cells. J. Membr. Biol., 188(2), 137-49. Retrieved from http://link.springer.com/10.1007/s00232-001 -0181-3 doi: 10.1007/s00232-001-0181-3

Gavrilova-Ruch, O., Schönherr, R., \& Heinemann, S. H. (2007, mar). Activation of hEAG1 potassium channels by arachidonic acid. Pflugers Arch., 453(6), 891-903. Retrieved from http://link.springer.com/10.1007/s00424-006 -0173-3 doi: 10.1007/s00424-006-0173-3

Gee, K., Brown, K., Chen, W.-N., Bishop-Stewart, J., Gray, D., \& Johnson, I. (2000, feb). Chemical and physiological characterization of fluo-4 Ca2+-indicator dyes. 


\section{REFERENCES}

Cell Calcium, 27(2), 97-106. Retrieved from http://www.ncbi.nlm.nih.gov/ pubmed/10756976 doi: 10.1054/ceca.1999.0095

Gierke, S., Kumar, P., \& Wittmann, T. (2010). Analysis of microtubule polymerization dynamics in live cells. Methods Cell Biol., 97, 15-33. Retrieved from http://www.ncbi.nlm.nih.gov/pubmed/20719263 doi: 10.1016/S0091 $-679 \mathrm{X}(10) 97002-7$

Gilberto, S., \& Peter, M. (2017, aug). Dynamic ubiquitin signaling in cell cycle regulation. J. Cell Biol., 216(8), 2259-2271. Retrieved from http://www.ncbi .nlm.nih.gov/pubmed/28684425 doi: 10.1083/jcb.201703170

Girard, F., Strausfeld, U., Fernandez, A., \& Lamb, N. J. (1991, dec). Cyclin A is required for the onset of DNA replication in mammalian fibroblasts. Cell, 67(6), 1169-79. Retrieved from http://www.ncbi.nlm.nih.gov/pubmed/1836977

Glaubke, E., \& Bastians, H. (2018). A Cell-Based Assay for Mitotic Spindle Orientation. In Methods mol. biol. (Vol. 1787, pp. 67-75). Retrieved from http://www.ncbi.nlm.nih.gov/pubmed/29736710 doi: 10.1007/978-1-4939 $-7847-2 \_5$

Golan, A., Pick, E., Tsvetkov, L., Nadler, Y., Kluger, H., \& Stern, D. F. (2010, jul). Centrosomal Chk2 in DNA damage responses and cell cycle progession. Cell Cycle, 9(13), 2647-2656. Retrieved from http://www.ncbi.nlm.nih.gov/ pubmed/20581449 doi: 10.4161/cc.9.13.12121

Golan, A., Yudkovsky, Y., \& Hershko, A. (2002, may). The Cyclin-Ubiquitin Ligase Activity of Cyclosome/APC Is Jointly Activated by Protein Kinases Cdk1-Cyclin B and Plk. J. Biol. Chem., 277(18), 15552-15557. Retrieved from http://www.ncbi.nlm.nih.gov/pubmed/11859075 doi: 10.1074/jbc .M111476200

Gómez-Varela, D., Kohl, T., Schmidt, M., Rubio, M. E., Kawabe, H., Nehring, R. B., .. Pardo, L. A. (2010, jan). Characterization of Eag1 channel lateral mobility in rat hippocampal cultures by single-particle-tracking with quantum dots. PLoS One, 5(1), e8858. Retrieved from https://dx.plos.org/10.1371/ journal.pone.0008858 doi: 10.1371/journal.pone.0008858

Gomez-Varela, D., Zwick-Wallasch, E., Knotgen, H., Sanchez, A., Hettmann, T., Ossipov, D., ... Pardo, L. A. (2007, aug). Monoclonal Antibody Blockade of the 
Human Eag1 Potassium Channel Function Exerts Antitumor Activity. Cancer Res., 67(15), 7343-7349. Retrieved from http://www.ncbi.nlm.nih.gov/ pubmed/17671204 doi: 10.1158/0008-5472.CAN-07-0107

Gonçalves, J. T., \& Stühmer, W. (2010). Calmodulin Interaction with hEAG1 Visualized by FRET Microscopy. PLoS One, 5(5), 1-8. Retrieved from https://doi.org/10.1371/journal.pone.0010873 doi: 10.1371/journal .pone.0010873

Gong, D., Pomerening, J. R., Myers, J. W., Gustavsson, C., Jones, J. T., Hahn, A. T., .. Ferrell, J. E. (2007, jan). Cyclin A2 regulates nuclear-envelope breakdown and the nuclear accumulation of cyclin B1. Curr. Biol., 17(1), 85-91. Retrieved from https://linkinghub.elsevier.com/retrieve/pii/ S0960982206025863 doi: 10.1016/j.cub.2006.11.066

Goodson, H. V., \& Jonasson, E. M. (2018, jun). Microtubules and MicrotubuleAssociated Proteins. Cold Spring Harb. Perspect. Biol., 10(6). Retrieved from http://cshperspectives.cshlp.org/content/10/6/a022608 .abstract doi: 10.1101/cshperspect.a022608

Grill, S. W., \& Hyman, A. A. (2005). Spindle Positioning by Cortical Pulling Forces. Dev. Cell, 8(4), 461-465. Retrieved from http://www.sciencedirect.com/ science/article/pii/S1534580705001048 doi: https://doi.org/10.1016/ j.devcel.2005.03.014

Gullberg, M., Gustafsdottir, S. M., Schallmeiner, E., Jarvius, J., Bjarnegard, M., Betsholtz, C., ... Fredriksson, S. (2004, jun). Cytokine detection by antibodybased proximity ligation. Proc. Natl. Acad. Sci., 101(22), 8420-8424. Retrieved from http://www.ncbi.nlm.nih.gov/pubmed/15155907 doi: 10.1073/pnas .0400552101

Gurling, H. M., Kalsi, G., Brynjolfson, J., Sigmundsson, T., Sherrington, R., Mankoo, B. S., .. Curtis, D. (2001, mar). Genomewide genetic linkage analysis confirms the presence of susceptibility loci for schizophrenia, on chromosomes 1q32.2, 5q33.2, and 8p21-22 and provides support for linkage to schizophrenia, on chromosomes 11q23.3-24 and 20q12.1-11.23. Am. J. Hum. Genet., 68(3), 66173. Retrieved from http://www.ncbi.nlm.nih.gov/pubmed/11179014 doi: $10.1086 / 318788$ 
Gutman, G. A., Chandy, K. G., Grissmer, S., Lazdunski, M., McKinnon, D., Pardo, L. A., .. Wang, X. (2005, dec). International Union of Pharmacology. LIII. Nomenclature and Molecular Relationships of Voltage-Gated Potassium Channels. Pharmacol. Rev., 57(4), 473-508. Retrieved from http://www.ncbi .nlm.nih.gov/pubmed/16382104 doi: 10.1124/pr.57.4.10

Haitin, Y., Carlson, A. E., \& Zagotta, W. N. (2013, sep). The structural mechanism of KCNH-channel regulation by the eag domain. Nature, 501(7467), 4448. Retrieved from http://www.nature.com/articles/nature12487 doi: 10.1038/nature12487

Hammadi, M., Chopin, V., Matifat, F., Dhennin-Duthille, I., Chasseraud, M., Sevestre, H., \& Ouadid-Ahidouch, H. (2012, dec). Human ether à-gogo $\mathrm{K}+$ channel 1 (hEag1) regulates MDA-MB-231 breast cancer cell migration through Orai1-dependent calcium entry. J. Cell. Physiol., 227(12), 38373846. Retrieved from http://www.ncbi.nlm.nih.gov/pubmed/22495877 doi: $10.1002 /$ jcp. 24095

Han, B., Tokay, T., Zhang, G., Sun, P., \& Hou, S. (2017). Eag1 K+ Channel: Endogenous Regulation and Functions in Nervous System. Oxid. Med. Cell. Longev., 2017, 7371010. Retrieved from http://www.ncbi.nlm.nih.gov/ pubmed/28367272 doi: 10.1155/2017/7371010

Hanafusa, H., Kedashiro, S., Tezuka, M., Funatsu, M., Usami, S., Toyoshima, F., \& Matsumoto, K. (2015, aug). PLK1-dependent activation of LRRK1 regulates spindle orientation by phosphorylating CDK5RAP2. Nat. Cell Biol., 17(8), 1024-1035. Retrieved from http://www.ncbi.nlm.nih.gov/pubmed/ 26192437 doi: 10.1038/ncb3204

Hanahan, D., \& Weinberg, R. A. (2011, mar). Hallmarks of Cancer: The Next Generation. Cell, 144(5), 646-674. Retrieved from http://www.ncbi.nlm .nih.gov/pubmed/21376230 doi: 10.1016/j.cell.2011.02.013

Harper, J. V. (2005). Synchronization of cell populations in G1/S and G2/M phases of the cell cycle. Methods Mol. Biol., 296, 157-66. Retrieved from http://www.ncbi.nlm.nih.gov/pubmed/15576930

Harper, J. W., \& Elledge, S. J. (2007, dec). The DNA Damage Response: Ten Years After. Mol. Cell, 28(5), 739-745. Retrieved from https://doi.org/10.1016/ 
j.molcel.2007.11.015 doi: 10.1016/j.molcel.2007.11.015

Hartung, F., \& Pardo, L. A. (2016). Guiding TRAIL to cancer cells through Kv10.1 potassium channel overcomes resistance to doxorubicin. Eur. Biophys. J., 45(7), 709-719. Retrieved from https://doi.org/10.1007/s00249-016 -1149-7 doi: 10.1007/s00249-016-1149-7

Hartung, F., Stühmer, W., \& Pardo, L. A. (2011, sep). Tumor cell-selective apoptosis induction through targeting of $\mathrm{K}(\mathrm{V}) 10.1$ via bifunctional TRAIL antibody. Mol. Cancer, 10, 109. Retrieved from http://www.ncbi.nlm.nih.gov/pubmed/ 21899742 doi: 10.1186/1476-4598-10-109

Heald, R., \& Khodjakov, A. (2015, dec). Thirty years of search and capture: The complex simplicity of mitotic spindle assembly. J. Cell Biol., 211(6), 1103 LP - 1111. Retrieved from http://jcb.rupress.org/content/211/6/ 1103.abstract doi: $10.1083 /$ jcb.201510015

Heerboth, S., Housman, G., Leary, M., Longacre, M., Byler, S., Lapinska, K., ... Sarkar, S. (2015). EMT and tumor metastasis. Clin. Transl. Med., 4, 6. Retrieved from http://www.ncbi.nlm.nih.gov/pubmed/25852822 doi: $10.1186 / \mathrm{s} 40169-015-0048-3$

Hell, M. P., Duda, M., Weber, T. C., Moch, H., \& Krek, W. (2014, may). Tumor Suppressor VHL Functions in the Control of Mitotic Fidelity. Cancer Res., 74 (9), 2422-2431. Retrieved from http://www.ncbi.nlm.nih.gov/pubmed/ 24362914 doi: 10.1158/0008-5472.CAN-13-2040

Hemmerlein, B., Weseloh, R. M., Mello de Queiroz, F., Knötgen, H., Sánchez, A., Rubio, M. E., ... Pardo, L. A. (2006, oct). Overexpression of Eag1 potassium channels in clinical tumours. Mol. Cancer, 5, 41. Retrieved from http:// www.ncbi.nlm.nih.gov/pubmed/17022810 doi: 10.1186/1476-4598-5-41

Henglein, B., Chenivesse, X., Wang, J., Eick, D., \& Bréchot, C. (1994, jun). Structure and cell cycle-regulated transcription of the human cyclin A gene. Proc. Natl. Acad. Sci. U. S. A., 91(12), 5490-4. Retrieved from http:// www.ncbi.nlm.nih.gov/pubmed/8202514

Hepler, P. K. (1985, may). Calcium restriction prolongs metaphase in dividing Tradescantia stamen hair cells. J. Cell Biol., 100(5), 1363-8. Retrieved from http://www.ncbi.nlm.nih.gov/pubmed/3921550 doi: 10.1083/JCB.100.5 


\section{REFERENCES}

.1363

Hepler, P. K. (2016, jan). The Cytoskeleton and Its Regulation by Calcium and Protons. Plant Physiol., 170(1), 3-22. Retrieved from http://www.ncbi.nlm .nih.gov/pubmed/26722019 doi: 10.1104/pp.15.01506

Hergovich, A., Lisztwan, J., Barry, R., Ballschmieter, P., \& Krek, W. (2002, dec). Regulation of microtubule stability by the von Hippel-Lindau tumour suppressor protein pVHL. Nat. Cell Biol., 5(1), 64-70. Retrieved from http://www.ncbi.nlm.nih.gov/pubmed/12510195 doi: 10.1038/ncb899

Herrmann, S., Ninkovic, M., Kohl, T., Lörinczi, É., \& Pardo, L. A. (2012, dec). Cortactin controls surface expression of the voltage-gated potassium channel K(V)10.1. J. Biol. Chem., 287(53), 44151-63. Retrieved from http://www .jbc.org/lookup/doi/10.1074/jbc.M112.372540 doi: 10.1074/jbc.M112 .372540

Hildebrandt, F., Benzing, T., \& Katsanis, N. (2011, apr). Ciliopathies. N. Engl. J. Med., 364(16), 1533-1543. Retrieved from http://www.ncbi.nlm.nih.gov/ pubmed/21506742 doi: 10.1056/NEJMra1010172

Hille, B. (2001). Ion channels of excitable membranes. Sinauer. Retrieved from https://books.google.de/books?id=8Vk-QwAACAAJ $\{\&\}$ dq=Ionic + channelstof+excitable+membranes $+2001\{\&\}$ hl $=e n\{\&\}$ sa $=X\{\&\}$ ved $=$ OahUKEwiP8YbM34fhAhVBaFAKHcekCmcQ6AEIKjAA

Hindi, S. M., Tajrishi, M. M., \& Kumar, A. (2013, apr). Signaling Mechanisms in Mammalian Myoblast Fusion. Sci. Signal., 6(272), re2-re2. Retrieved from http://www.ncbi.nlm.nih.gov/pubmed/23612709 doi: 10.1126/scisignal .2003832

Hitomi, M., \& Stacey, D. W. (1999, oct). Cyclin D1 production in cycling cells depends on ras in a cell-cycle-specific manner. Curr. Biol., 9(19), 1075-84. Retrieved from http://www.ncbi.nlm.nih.gov/pubmed/10531005

Hoebeke, J., Van Nijen, G., \& De Brabander, M. (1976, mar). Interaction of oncodazole (R 17934), a new antitumoral drug, with rat brain tubulin. Biochem. Biophys. Res. Commun., 69(2), 319-24. Retrieved from http://www.ncbi .nlm.nih.gov/pubmed/1267789

Holland, A. J., \& Cleveland, D. W. (2009, jul). Boveri revisited: chromosomal 
instability, aneuploidy and tumorigenesis. Nat. Rev. Mol. Cell Biol., 10(7), 478-87. Retrieved from http://www.nature.com/articles/nrm2718 doi: $10.1038 / \mathrm{nrm} 2718$

Huang, S., \& Zhang, C. (2011, aug). The Coordination between DNA Replication Initiation and Other Cell Cycle Events. In Dna replicationcurrent adv. InTech. Retrieved from http://www.intechopen.com/ books/dna-replication-current-advances/the-coordination-between -dna-replication-initiation-and-other-cell-cycle-events doi: $10.5772 / 21646$

Humeau, J., Bravo-San Pedro, J. M., Vitale, I., Nuñez, L., Villalobos, C., Kroemer, G., \& Senovilla, L. (2018, mar). Calcium signaling and cell cycle: Progression or death. Cell Calcium, 70, 3-15. Retrieved from https://www.sciencedirect .com/science/article/pii/S0143416017300611\{\#\}tbl0005 doi: 10.1016/ J.CECA.2017.07.006

Hwang, S. G., Lee, D., Kim, J., Seo, T., \& Choe, J. (2002, jan). Human Papillomavirus Type 16 E7 Binds to E2F1 and Activates E2F1-driven Transcription in a Retinoblastoma Protein-independent Manner. J. Biol. Chem., 277(4), 29232930. Retrieved from http://www.ncbi.nlm.nih.gov/pubmed/11713253 doi: 10.1074/jbc.M109113200

Ikeda, M., \& Tanaka, K. (2017, dec). Plk1 bound to Bub1 contributes to spindle assembly checkpoint activity during mitosis. Sci. Rep., 7(1), 8794. Retrieved from http://www.ncbi.nlm.nih.gov/pubmed/28821799 doi: 10.1038/s41598 $-017-09114-3$

Illingworth, C., Pirmadjid, N., Serhal, P., Howe, K., \& Fitzharris, G. (2010, jul). MCAK regulates chromosome alignment but is not necessary for preventing aneuploidy in mouse oocyte meiosis I. Development, 137(13), 2133-8. Retrieved from http://www.ncbi.nlm.nih.gov/pubmed/20504960 doi: 10.1242/dev .048306

Ireland, C. M., Gull, K., Gutteridge, W. E., \& Pogson, C. I. (1979, sep). The interaction of benzimidazole carbamates with mammalian microtobule protein. Biochem. Pharmacol., 28(17), 2680-2. Retrieved from http://www.ncbi.nlm .nih.gov/pubmed/518680 


\section{REFERENCES}

Iyer, V. R., Eisen, M. B., Ross, D. T., Schuler, G., Moore, T., Lee, J. C., ... Brown, P. O. (1999, jan). The transcriptional program in the response of human fibroblasts to serum. Science, 283(5398), 83-7. Retrieved from http://www.ncbi.nlm.nih.gov/pubmed/9872747

Izant, J. G. (1983). The role of calcium ions during mitosis. Chromosoma, 88(1), 1-10. Retrieved from https://doi.org/10.1007/BF00329497 doi: 10.1007/ BF00329497

Jackman, M., Kubota, Y., den Elzen, N., Hagting, A., \& Pines, J. (2002, mar). Cyclin A- and cyclin E-Cdk complexes shuttle between the nucleus and the cytoplasm. Mol. Biol. Cell, 13(3), 1030-45. Retrieved from http://www.ncbi .nlm.nih.gov/pubmed/11907280 doi: 10.1091/mbc.01-07-0361

Jaqaman, K., Loerke, D., Mettlen, M., Kuwata, H., Grinstein, S., Schmid, S. L., \& Danuser, G. (2008, aug). Robust single-particle tracking in live-cell time-lapse sequences. Nat. Methods, 5(8), 695-702. Retrieved from http://www. nature .com/doifinder/10.1038/nmeth.1237 doi: 10.1038/nmeth.1237

Jeng, C.-J., Chang, C.-C., \& Tang, C.-Y. (2005, feb). Differential localization of rat Eag1 and Eag2 K+ channels in hippocampal neurons. Neuroreport, 16(3), 229-33. Retrieved from http://www.ncbi.nlm.nih.gov/pubmed/15706225

Jenke, M., Sánchez, A., Monje, F., Stühmer, W., Weseloh, R. M., \& Pardo, L. A. (2003, feb). C-terminal domains implicated in the functional surface expression of potassium channels. EMBO J., 22(3), 395-403. Retrieved from http://emboj.embopress.org/cgi/doi/10.1093/emboj/cdg035 doi: 10.1093/emboj/cdg035

Jia, L., Li, B., \& Yu, H. (2016, dec). The Bub1-Plk1 kinase complex promotes spindle checkpoint signalling through Cdc20 phosphorylation. Nat. Commun., 7(1), 10818. Retrieved from http://www.ncbi.nlm.nih.gov/pubmed/26912231 doi: $10.1038 /$ ncomms10818

Jiang, K., Pereira, E., Maxfield, M., Russell, B., Goudelock, D. M., \& Sanchez, Y. (2003, jul). Regulation of Chk1 Includes Chromatin Association and 14-3-3 Binding following Phosphorylation on Ser-345. J. Biol. Chem., 278(27), 2520725217. Retrieved from http://www.ncbi.nlm.nih.gov/pubmed/12676962 doi: 10.1074/jbc.M300070200 
Jiang, X. R., Jimenez, G., Chang, E., Frolkis, M., Kusler, B., Sage, M., ... Chiu, C. P. (1999, jan). Telomerase expression in human somatic cells does not induce changes associated with a transformed phenotype. Nat. Genet., 21(1), 111-4. Retrieved from http://www.ncbi.nlm.nih.gov/pubmed/9916802 doi: $10.1038 / 5056$

Jongsma, M. L. M., Berlin, I., \& Neefjes, J. (2015, mar). On the move: organelle dynamics during mitosis. Trends Cell Biol., 25(3), 112-124. Retrieved from https://doi.org/10.1016/j.tcb.2014.10.005 doi: 10.1016/j.tcb.2014.10 .005

Jordan, A., \& Reichard, P. (1998, jun). RIBONUCLEOTIDE REDUCTASES. Annu. Rev. Biochem., 67(1), 71-98. Retrieved from http://www.ncbi.nlm.nih.gov/ pubmed/9759483 doi: 10.1146/annurev.biochem.67.1.71

Jow, G.-M., \& Jeng, C.-J. (2008, jan). Differential localization of rat Eag1 and Eag2 potassium channels in the retina. Neurosci. Lett., 431(1), 1216. Retrieved from http://www.ncbi.nlm.nih.gov/pubmed/18063306 doi: 10.1016/j.neulet.2007.11.017

Ju, M., \& Wray, D. (2002, jul). Molecular identification and characterisation of the human eag2 potassium channel. FEBS Lett., 524(1-3), 204-10.

Kahl, C. R., \& Means, A. R. (2003, dec). Regulation of Cell Cycle Progression by Calcium/Calmodulin-Dependent Pathways. Endocr. Rev., 24(6), 719-736. Retrieved from https://doi.org/10.1210/er.2003-0008 doi: 10.1210/er $.2003-0008$

Kamb, A., Tseng-Crank, J., \& Tanouye, M. A. (1988, jul). Multiple products of the Drosophila Shaker gene may contribute to potassium channel diversity. Neuron, 1(5), 421-30. Retrieved from http://www.ncbi.nlm.nih.gov/pubmed/ 3272175

Kao, J. P., Alderton, J. M., Tsien, R. Y., \& Steinhardt, R. A. (1990, jul). Active involvement of $\mathrm{Ca} 2+$ in mitotic progression of Swiss $3 \mathrm{~T} 3$ fibroblasts. $J$. Cell Biol., 111(1), 183-96. Retrieved from http://www.ncbi.nlm.nih.gov/ pubmed/2114410 doi: 10.1083/JCB.111.1.183

Kaplan, W. D., \& Trout, W. E. (1969, feb). The behavior of four neurological mutants of Drosophila. Genetics, 61(2), 399-409. Retrieved from http:// 


\section{REFERENCES}

www.ncbi.nlm.nih.gov/pubmed/5807804

Kastan, M. B., \& Bartek, J. (2004, nov). Cell-cycle checkpoints and cancer. Nature, 432(7015), 316-23. Retrieved from http://www.nature.com/articles/ nature03097 doi: 10.1038/nature03097

Kastan, M. B., \& Lim, D.-s. (2000, dec). The many substrates and functions of ATM. Nat. Rev. Mol. Cell Biol., 1(3), 179-186. Retrieved from http:// www.ncbi.nlm.nih.gov/pubmed/11252893 doi: 10.1038/35043058

Kidiyoor, G. R., Kumar, A., \& Foiani, M. (2016, aug). ATR-mediated regulation of nuclear and cellular plasticity. DNA Repair (Amst)., 44, 143150. Retrieved from http://www.ncbi.nlm.nih.gov/pubmed/27283761 doi: 10.1016/j.dnarep.2016.05.020

Kim, T. K., \& Eberwine, J. H. (2010, aug). Mammalian cell transfection: the present and the future. Anal. Bioanal. Chem., 397(8), 3173-8. Retrieved from http:// WwW.ncbi.nlm.nih.gov/pubmed/20549496 doi: 10.1007/s00216-010-3821-6

King, R. W., Peters, J. M., Tugendreich, S., Rolfe, M., Hieter, P., \& Kirschner, M. W. (1995, apr). A 20S complex containing CDC27 and CDC16 catalyzes the mitosis-specific conjugation of ubiquitin to cyclin B. Cell, 81(2), 279-88. Retrieved from http://www.ncbi.nlm.nih.gov/pubmed/7736580

Kirschner, M., \& Mitchison, T. (1986). Beyond self-assembly: from microtubules to morphogenesis. Cell, 45(3), 329-342.

Kiyomitsu, T., \& Cheeseman, I. M. (2012, mar). Chromosome- and spindle-polederived signals generate an intrinsic code for spindle position and orientation. Nat. Cell Biol., 14(3), 311-317. Retrieved from http://www.ncbi.nlm.nih .gov/pubmed/22327364 doi: 10.1038/ncb2440

Knapp, D. J., Breese, C. R., Mueller, R. A., \& Breese, G. R. (2001, apr). Drugs and the Synapse. In Encycl. life sci. Chichester: John Wiley \& Sons, Ltd. Retrieved from http://doi.wiley.com/10.1038/npg.els.0000098 doi: 10 $.1038 /$ npg.els.0000098

Końca, K. (2015). Documentation - CaspLab. Retrieved from http://casplab . $\mathrm{com}$

Końca, K., Lankoff, A., Banasik, A., Lisowska, H., Kuszewski, T., Góźdź, S., ... Wojcik, A. (2003, jan). A cross-platform public domain PC image-analysis 
program for the comet assay. Mutat. Res., 534(1-2), 15-20. Retrieved from http://www. casp.of.pl.

Koong, A. C., Denko, N. C., Hudson, K. M., Schindler, C., Swiersz, L., Koch, C., ... Giaccia, A. J. (2000, feb). Candidate genes for the hypoxic tumor phenotype. Cancer Res., 60(4), 883-7. Retrieved from http://www.ncbi.nlm.nih.gov/ pubmed/10706099

Kotak, S. (2019, feb). Mechanisms of Spindle Positioning: Lessons from Worms and Mammalian Cells. Biomolecules, 9(2), 80. Retrieved from http:// www.ncbi.nlm.nih.gov/pubmed/30823600 doi: 10.3390/biom9020080

Kraft, C., Herzog, F., Gieffers, C., Mechtler, K., Hagting, A., Pines, J., \& Peters, J.M. (2003, dec). Mitotic regulation of the human anaphase-promoting complex by phosphorylation. EMBO J., 22(24), 6598-609. Retrieved from http:// www.ncbi.nlm.nih.gov/pubmed/14657031 doi: 10.1093/emboj/cdg627

Krämer, A., Mailand, N., Lukas, C., Syljuåsen, R. G., Wilkinson, C. J., Nigg, E. A., ... Lukas, J. (2004, aug). Centrosome-associated Chk1 prevents premature activation of cyclin-B-Cdk1 kinase. Nat. Cell Biol., 6, 884. Retrieved from https://doi.org/10.1038/ncb1165

Krenn, V., \& Musacchio, A. (2015). The Aurora B Kinase in Chromosome BiOrientation and Spindle Checkpoint Signaling. Front. Oncol., 5, 225. Retrieved from http://www.ncbi.nlm.nih.gov/pubmed/26528436 doi: 10.3389/fonc .2015 .00225

Lai, Q., Wang, T., Guo, Q., Zhang, Y., Wang, Y., Yuan, L., .. Wang, W. (2014, may). Positive correlation between the expression of hEag1 and HIF-1 $\alpha$ in breast cancers: an observational study. BMJ Open, 4(5), e005049. Retrieved from http://bmjopen.bmj.com/lookup/doi/10.1136/bmjopen-2014 -005049 doi: 10.1136/bmjopen-2014-005049

Lampson, M. A., Renduchitala, K., Khodjakov, A., \& Kapoor, T. M. (2004, mar). Correcting improper chromosome-spindle attachments during cell division. Nat. Cell Biol., 6(3), 232-237. Retrieved from http://www.ncbi.nlm.nih.gov/ pubmed/14767480 doi: 10.1038/ncb1102

Lang, F., Föller, M., Lang, K., Lang, P., Ritter, M., Vereninov, A., ... Gulbins, E. (2007). Cell Volume Regulatory Ion Channels in Cell Proliferation 


\section{REFERENCES}

and Cell Death. In Methods enzymol. (Vol. 428, pp. 209-225). Retrieved from http://www.ncbi.nlm.nih.gov/pubmed/17875419 doi: 10.1016/S0076 $-6879(07) 28011-5$

Lang, F., \& Stournaras, C. (2014, feb). Ion channels in cancer: future perspectives and clinical potential. Philos. Trans. R. Soc. B Biol. Sci., 369(1638), 20130108 20130108. Retrieved from http://www.ncbi.nlm.nih.gov/pubmed/24493756 doi: $10.1098 /$ rstb.2013.0108

Langie, S. A. S., Azqueta, A., \& Collins, A. R. (2015). The comet assay: past, present, and future. Front. Genet., 6, 266. Retrieved from http://www.ncbi .nlm.nih.gov/pubmed/26322077 doi: 10.3389/fgene.2015.00266

Lansbergen, G., \& Akhmanova, A. (2006, may). Microtubule Plus End: A Hub of Cellular Activities. Traffic, 7(5), 499-507. Retrieved from http://doi.wiley $. c o m / 10.1111 / j .1600-0854.2006 .00400 . x$ doi: 10.1111/j.1600-0854.2006 $.00400 . \mathrm{x}$

Lara-Gonzalez, P., Westhorpe, F. G., \& Taylor, S. S. (2012, nov). The Spindle Assembly Checkpoint. Curr. Biol., 22(22), R966-R980. Retrieved from http:// www.ncbi.nlm.nih.gov/pubmed/23174302 doi: 10.1016/j.cub.2012.10.006

Larijani, B., \& Poccia, D. L. (2009, jun). Nuclear envelope formation: mind the gaps. Annu. Rev. Biophys., 38(1), 107-24. Retrieved from http://www .annualreviews.org/doi/10.1146/annurev.biophys.050708.133625 doi: 10.1146/annurev.biophys.050708.133625

Lawrence, K. S., Chau, T., \& Engebrecht, J. (2015, apr). DNA Damage Response and Spindle Assembly Checkpoint Function throughout the Cell Cycle to Ensure Genomic Integrity. PLOS Genet., 11(4), e1005150. Retrieved from http:// dx.plos.org/10.1371/journal.pgen.1005150 doi: 10.1371/journal.pgen .1005150

Leanza, L., Henry, B., Sassi, N., Zoratti, M., Chandy, K. G., Gulbins, E., \& Szabò, I. (2012, jul). Inhibitors of mitochondrial Kv1.3 channels induce Bax/Bak-independent death of cancer cells. EMBO Mol. Med., 4(7), 577. Retrieved from http://www.ncbi.nlm.nih.gov/pubmed/22496117 doi: 10 $.1002 /$ EMMM.201200235

Leanza, L., Romio, M., Becker, K. A., Azzolini, M., Trentin, L., Managò, A., 
.. Szabo, I. (2017, apr). Direct Pharmacological Targeting of a Mitochondrial Ion Channel Selectively Kills Tumor Cells In Vivo. Cancer Cell, 31(4), 516-531.e10. Retrieved from https://www.sciencedirect.com/ science/article/pii/S1535610817300995?via\{\%\}3Dihub doi: 10.1016/ J.CCELL.2017.03.003

Leanza, L., Venturini, E., Kadow, S., Carpinteiro, A., Gulbins, E., \& Becker, K. A. (2015, jul). Targeting a mitochondrial potassium channel to fight cancer. Cell Calcium, 58(1), 131-138. Retrieved from http://www.ncbi.nlm.nih.gov/ pubmed/25443654 doi: 10.1016/j.ceca.2014.09.006

Lee, D. H., \& Goldberg, A. L. (1996, nov). Selective inhibitors of the proteasomedependent and vacuolar pathways of protein degradation in Saccharomyces cerevisiae. J. Biol. Chem., 271(44), 27280-4. Retrieved from http://www .ncbi.nlm.nih.gov/pubmed/8910302

Lee, D. H., \& Goldberg, A. L. (1998, oct). Proteasome inhibitors: valuable new tools for cell biologists. Trends Cell Biol., 8(10), 397-403. Retrieved from http://www.ncbi.nlm.nih.gov/pubmed/9789328

Lee, J. C., Field, D. J., \& Lee, L. L. (1980, dec). Effects of nocodazole on structures of calf brain tubulin. Biochemistry, 19(26), 6209-15. Retrieved from http://www.ncbi.nlm.nih.gov/pubmed/7470461

Lee, J.-H., \& Paull, T. T. (2005, apr). ATM Activation by DNA Double-Strand Breaks Through the Mre11-Rad50-Nbs1 Complex. Science (80-. )., 308(5721), 551-554. Retrieved from http://www.ncbi.nlm.nih.gov/pubmed/15790808 doi: $10.1126 /$ science.1108297

Lee, Y. C., \& Wolff, J. (1982, jun). Two opposing effects of calmodulin on microtubule assembly depend on the presence of microtubule-associated proteins. J. Biol. Chem., 257(11), 6306-10. Retrieved from http://www.ncbi.nlm.nih.gov/ pubmed/6804463

Lera, R. F., \& Burkard, M. E. (2012, dec). High Mitotic Activity of Polo-like Kinase 1 Is Required for Chromosome Segregation and Genomic Integrity in Human Epithelial Cells. J. Biol. Chem., 287(51), 42812-42825. Retrieved from http:// www.ncbi.nlm.nih.gov/pubmed/23105120 doi: 10.1074/jbc.M112.412544

Li, C., \& Balazsi, G. (2018). A landscape view on the interplay between EMT and 


\section{REFERENCES}

cancer metastasis. npj Syst. Biol. Appl., 4(1), 34. Retrieved from https:// doi.org/10.1038/s41540-018-0068-x doi: 10.1038/s41540-018-0068-x

Lianga, N., Williams, E. C., Kennedy, E. K., Doré, C., Pilon, S., Girard, S. L., ... Rudner, A. D. (2013, jun). A Wee1 checkpoint inhibits anaphase onset. $J$. Cell Biol., 201(6), 843-62. Retrieved from http://www.jcb.org/lookup/doi/ $10.1083 /$ jcb. 201212038 doi: 10.1083/jcb.201212038

Lin, H., Li, Z., Chen, C., Luo, X., Xiao, J., Dong, D., ... Wang, Z. (2011, may). Transcriptional and Post-Transcriptional Mechanisms for Oncogenic Overexpression of Ether À Go-Go K+ Channel. PLoS One, 6(5), e20362. Retrieved from http://dx.plos.org/10.1371/journal.pone.0020362 doi: 10.1371/journal.pone.0020362

Lin, S.-Y., Li, K., Stewart, G. S., \& Elledge, S. J. (2004, apr). Human Claspin works with BRCA1 to both positively and negatively regulate cell proliferation. Proc. Natl. Acad. Sci., 101(17), 6484-6489. Retrieved from http://www.ncbi.nlm .nih.gov/pubmed/15096610 doi: 10.1073/pnas.0401847101

Lindqvist, A., Rodríguez-Bravo, V., \& Medema, R. H. (2009). The decision to enter mitosis: feedback and redundancy in the mitotic entry network. J. Cell Biol., $185(2), 193-202$.

Liu, J. H., Bijlenga, P., Fischer-Lougheed, J., Occhiodoro, T., Kaelin, A., Bader, C. R., \& Bernheim, L. (1998, jul). Role of an inward rectifier K+ current and of hyperpolarization in human myoblast fusion. J. Physiol., 510 ( Pt 2(Pt 2), 467-76. Retrieved from http://www.ncbi.nlm.nih.gov/pubmed/9705997 doi: 10.1111/J.1469-7793.1998.467BK.X

Liu, R.-G., Wang, W.-J., Song, N., Chen, Y.-Q., \& Li, L.-H. (2006, aug). Diazoxide preconditioning alleviates apoptosis of hippocampal neurons induced by anoxiareoxygenation in vitro through up-regulation of Bcl-2/Bax protein ratio. Sheng Li Xие Bao, 58(4), 345-50. Retrieved from http://www.ncbi.nlm.nih.gov/ pubmed/16906335

Lodish, H. F. (2000). Molecular cell biology. W.H. Freeman. Retrieved from https://www.ncbi.nlm.nih.gov/books/NBK21475/

Lolkema, M. P., Mans, D. A., Snijckers, C. M., van Noort, M., van Beest, M., Voest, E. E., \& Giles, R. H. (2007, oct). The von Hippel-Lindau tumour suppressor 
interacts with microtubules through kinesin-2. FEBS Lett., 581 (24), 45714576. Retrieved from http://doi.wiley.com/10.1016/j.febslet.2007.08 .050 doi: 10.1016/j.febslet.2007.08.050

Lolkema, M. P., Mehra, N., Jorna, A. S., van Beest, M., Giles, R. H., \& Voest, E. E. (2004, dec). The von Hippel?Lindau tumor suppressor protein influences microtubule dynamics at the cell periphery. Exp. Cell Res., 301(2), 139-146. Retrieved from http://linkinghub.elsevier.com/retrieve/pii/ S0014482704003945 doi: 10.1016/j.yexcr.2004.07.016

Long, S. B., Campbell, E. B., \& Mackinnon, R. (2005a, aug). Crystal Structure of a Mammalian Voltage-Dependent Shaker Family K+ Channel. Science (80-. )., 309(5736), 897-903. Retrieved from http://www.ncbi.nlm.nih.gov/pubmed/ 16002581 doi: 10.1126/science.1116269

Long, S. B., Campbell, E. B., \& Mackinnon, R. (2005b, aug). Voltage Sensor of Kv1.2: Structural Basis of Electromechanical Coupling. Science (80-. )., 309(5736), 903-908. Retrieved from http://www.ncbi.nlm.nih.gov/pubmed/16002579 doi: $10.1126 /$ science. 1116270

Long, S. B., Tao, X., Campbell, E. B., \& MacKinnon, R. (2007, nov). Atomic structure of a voltage-dependent $\mathrm{K}+$ channel in a lipid membrane-like environment. Nature, 450(7168), 376-382. Retrieved from http://www.ncbi.nlm.nih.gov/ pubmed/18004376 doi: 10.1038/nature06265

Lorenzo, C., Liao, Q., Hardwicke, M. A., \& Ducommun, B. (2009, jun). Pharmacological inhibition of Aurora-A but not Aurora-B impairs interphase microtubule dynamics. Cell Cycle, 8(11), 1733-1737. Retrieved from http:// www.ncbi.nlm.nih.gov/pubmed/19395863 doi: 10.4161/cc.8.11.8617

Lörinczi, E., Helliwell, M., Finch, A., Stansfeld, P. J., Davies, N. W., Mahaut-Smith, M., .. Mitcheson, J. S. (2016, aug). Calmodulin Regulates Human Ether à Go-Go 1 (hEAG1) Potassium Channels through Interactions of the Eag Domain with the Cyclic Nucleotide Binding Homology Domain. J. Biol. Chem., 291(34), 17907-17918. Retrieved from http://www.jbc.org/content/291/ 34/17907. abstract

Luers, H. (1936). Shaker, eine erbliche Bewegungsstorung bei Drosophila funebris. 2. Ind. Abst. Vererb, 72, 119-126. 


\section{REFERENCES}

Lukas, C., Bartkova, J., Latella, L., Falck, J., Mailand, N., Schroeder, T., ... Bartek, J. (2001, jul). DNA Damage-activated Kinase Chk2 Is Independent of Proliferation or Differentiation Yet Correlates with Tissue Biology. Cancer Res., 61(13), 4990 LP - 4993. Retrieved from http://cancerres.aacrjournals . org/content/61/13/4990. abstract

Ma, H. T., \& Poon, R. Y. (2011). Synchronization of HeLa Cells. In Methods mol. biol. (Vol. 761, pp. 151-161). Retrieved from http://link.springer.com/ 10.1007/978-1-61779-182-6\{_\}10 doi: 10.1007/978-1-61779-182-6_10

Macaluso, M., Montanari, M., \& Giordano, A. (2006, aug). Rb family proteins as modulators of gene expression and new aspects regarding the interaction with chromatin remodeling enzymes. Oncogene, 25(38), 5263-7. Retrieved from http://www.nature.com/articles/1209680 doi: 10.1038/sj.onc.1209680

Macůrek, L., Lindqvist, A., Lim, D., Lampson, M. A., Klompmaker, R., Freire, R., ... Medema, R. H. (2008). Polo-like kinase-1 is activated by aurora A to promote checkpoint recovery. Nature, 455(7209), 119.

Macurek, L., Lindqvist, A., \& Medema, R. H. (2009, jun). Aurora-A and hBora Join the Game of Polo. Cancer Res., 69(11), 4555 LP - 4558. Retrieved from http: // cancerres.aacrjournals.org/content/69/11/4555.abstract doi: 10 .1158/0008-5472.CAN-09-0142

Maeno, E., Ishizaki, Y., Kanaseki, T., Hazama, A., \& Okada, Y. (2000, aug). Normotonic cell shrinkage because of disordered volume regulation is an early prerequisite to apoptosis. Proc. Natl. Acad. Sci., 97(17), 9487-9492. Retrieved from http://www.ncbi.nlm.nih.gov/pubmed/10900263 doi: 10.1073/pnas .140216197

Maney, T., Hunter, A. W., Wagenbach, M., \& Wordeman, L. (1998, aug). Mitotic centromere-associated kinesin is important for anaphase chromosome segregation. J. Cell Biol., 142(3), 787-801. Retrieved from http:// Www.ncbi.nlm.nih.gov/pubmed/9700166

Mann, B. J., \& Wadsworth, P. (2019, jan). Kinesin-5 Regulation and Function in Mitosis. Trends Cell Biol., 29(1), 66-79. Retrieved from http://www.ncbi .nlm.nih.gov/pubmed/30220581 doi: 10.1016/j.tcb.2018.08.004

Mapelli, M., Massimiliano, L., Santaguida, S., \& Musacchio, A. (2007, nov). The 
Mad2 Conformational Dimer: Structure and Implications for the Spindle Assembly Checkpoint. Cell, 131(4), 730-743. Retrieved from http://www .ncbi.nlm.nih.gov/pubmed/18022367 doi: 10.1016/j.cell.2007.08.049

Marques-Carvalho, M. J., Sahoo, N., Muskett, F. W., Vieira-Pires, R. S., Gabant, G., Cadene, M., ... Morais-Cabral, J. H. (2012, oct). Structural, biochemical, and functional characterization of the cyclic nucleotide binding homology domain from the mouse EAG1 potassium channel. J. Mol. Biol., 423(1), 34-46. Retrieved from https://linkinghub.elsevier.com/retrieve/pii/ S0022283612004986 doi: 10.1016/j.jmb.2012.06.025

Martin, B., Chesnel, F., Delcros, J.-G., Jouan, F., Couturier, A., Dugay, F., ... Arlot-Bonnemains, Y. (2013). Identification of pVHL as a Novel Substrate for Aurora-A in Clear Cell Renal Cell Carcinoma (ccRCC). PLoS One, 8(6), e67071. Retrieved from http://www.ncbi.nlm.nih.gov/pubmed/23785518 doi: 10.1371/journal.pone.0067071

Martin, S., Lino de Oliveira, C., Mello de Queiroz, F., Pardo, L., Stühmer, W., \& Del Bel, E. (2008, aug). Eag1 potassium channel immunohistochemistry in the CNS of adult rat and selected regions of human brain. Neuroscience, 155(3), 833-844. Retrieved from http://www.ncbi.nlm.nih.gov/pubmed/18650019 doi: 10.1016/j.neuroscience.2008.05.019

Martínez, R., Stühmer, W., Martin, S., Schell, J., Reichmann, A., Rohde, V., \& Pardo, L. (2015, nov). Analysis of the expression of Kv10.1 potassium channel in patients with brain metastases and glioblastoma multiforme: impact on survival. BMC Cancer, 15, 839. Retrieved from http://www.ncbi.nlm.nih .gov/pubmed/26530050 doi: 10.1186/s12885-015-1848-y

Martin-Romero, F. J., Lopez-Guerrero, A. M., Pascual-Caro, C., \& Pozo-Guisado, E. (2017, may). The Interplay between Cytoskeleton and Calcium Dynamics. In Cytoskelet. - struct. dyn. funct. dis. InTech. Retrieved from http://www.intechopen.com/ books/cytoskeleton-structure-dynamics-function-and-disease/ the-interplay-between-cytoskeleton-and-calcium-dynamics doi: $10.5772 / 66862$

Masters, J. R. (2002, apr). HeLa cells 50 years on: the good, the bad and the ugly. Nat. 


\section{REFERENCES}

Rev. Cancer, 2(4), 315-319. Retrieved from http://www.ncbi.nlm.nih.gov/ pubmed/12001993 doi: 10.1038/nrc775

Mathie, A., Wooltorton, J. R., \& Watkins, C. S. (1998, jan). Voltage-activated potassium channels in mammalian neurons and their block by novel pharmacological agents. Gen. Pharmacol., 30(1), 13-24. Retrieved from http:// www.ncbi.nlm.nih.gov/pubmed/9457476

Matov, A., Applegate, K., Kumar, P., Thoma, C., Krek, W., Danuser, G., \& Wittmann, T. (2010, sep). Analysis of microtubule dynamic instability using a plus-end growth marker. Nat. Methods, 7(9), 761-768. Retrieved from http:// www.ncbi.nlm.nih.gov/pubmed/20729842 doi: 10.1038/nmeth.1493

Matyskiela, M. E., \& Morgan, D. O. (2009, apr). Analysis of Activator-Binding Sites on the APC/C Supports a Cooperative Substrate-Binding Mechanism. Mol. Cell, 34(1), 68-80. Retrieved from https://doi.org/10.1016/j.molcel .2009.02.027 doi: 10.1016/j.molcel.2009.02.027

May, K. M., \& Hardwick, K. G. (2006, oct). The spindle checkpoint. J. Cell Sci., 119(20), 4139-4142. Retrieved from http://www.ncbi.nlm.nih.gov/pubmed/ 17038540 doi: $10.1242 /$ jcs.03165

Mayer, T. U., Kapoor, T. M., Haggarty, S. J., King, R. W., Schreiber, S. L., \& Mitchison, T. J. (1999, oct). Small molecule inhibitor of mitotic spindle bipolarity identified in a phenotype-based screen. Science, 286(5441), 971-4. Retrieved from http://www.ncbi.nlm.nih.gov/pubmed/10542155

Mayr, M. I., Hümmer, S., Bormann, J., Grüner, T., Adio, S., Woehlke, G., \& Mayer, T. U. (2007). The Human Kinesin Kif18A Is a Motile Microtubule Depolymerase Essential for Chromosome Congression. Curr. Biol., 17(6), 488498. Retrieved from http://www.sciencedirect.com/science/article/ pii/S0960982207010135 doi: https://doi.org/10.1016/j.cub.2007.02.036

Mazia, D. (1987). The chromosome cycle and the centrosome cycle in the mitotic cycle. Int. Rev. Cytol, 100, 49-92.

McIntosh, B. E., Hogenesch, J. B., \& Bradfield, C. A. (2010, mar). Mammalian PerArnt-Sim Proteins in Environmental Adaptation. Annu. Rev. Physiol., 72(1), 625-645. Retrieved from http://www.ncbi.nlm.nih.gov/pubmed/20148691 doi: 10.1146/annurev-physiol-021909-135922 
McNally, F. J. (2013). Mechanisms of spindle positioning. J. Cell Biol., 200(2). Retrieved from http://jcb.rupress.org/content/200/2/131

Mello de Queiroz, F., Suarez-Kurtz, G., Stühmer, W., \& Pardo, L. A. (2006, oct). Ether à go-go potassium channel expression in soft tissue sarcoma patients. Mol. Cancer, 5(1), 42. Retrieved from http://molecular-cancer.biomedcentral .com/articles/10.1186/1476-4598-5-42 doi: 10.1186/1476-4598-5-42

Menéndez, S. T., Villaronga, M. A., Rodrigo, J. P., Alvarez-Teijeiro, S., GarcíaCarracedo, D., Urdinguio, R. G., .. García-Pedrero, J. M. (2012, oct). Frequent aberrant expression of the human ether à go-go (hEAG1) potassium channel in head and neck cancer: pathobiological mechanisms and clinical implications. $J$. Mol. Med. (Berl)., 90(10), 1173-84. Retrieved from http://link.springer .com/10.1007/s00109-012-0893-0 doi: 10.1007/s00109-012-0893-0

Merriam, E. B., Millette, M., Lumbard, D. C., Saengsawang, W., Fothergill, T., Hu, X., .. Dent, E. W. (2013, oct). Synaptic Regulation of Microtubule Dynamics in Dendritic Spines by Calcium, F-Actin, and Drebrin. J. Neurosci., 33(42), 16471-16482. Retrieved from http://www.ncbi.nlm.nih.gov/ pubmed/24133252 doi: 10.1523/JNEUROSCI.0661-13.2013

Miller, M. P., Asbury, C. L., \& Biggins, S. (2016, jun). A TOG Protein Confers Tension Sensitivity to Kinetochore-Microtubule Attachments. Cell, 165(6), 14281439. Retrieved from http://www.ncbi.nlm.nih.gov/pubmed/27156448 doi: 10.1016/j.cell.2016.04.030

Minta, A., Kao, J. P., \& Tsien, R. Y. (1989, may). Fluorescent indicators for cytosolic calcium based on rhodamine and fluorescein chromophores. J. Biol. Chem., 264(14), 8171-8. Retrieved from http://www.ncbi.nlm.nih.gov/pubmed/ 2498308

Mirvis, M., Stearns, T., \& James Nelson, W. (2018, jul). Cilium structure, assembly, and disassembly regulated by the cytoskeleton. Biochem. J., 475(14), 2329 LP - 2353. Retrieved from http://www.biochemj.org/content/475/14/ 2329. abstract doi: 10.1042/BCJ20170453

Mitchison, T., \& Kirschner, M. (1984). Dynamic instability of microtubule growth. Nature, 312(5991), 237-242. Retrieved from https://doi.org/10.1038/ 312237a0 doi: 10.1038/312237a0 


\section{REFERENCES}

Mittal, V. (2018, jan). Epithelial Mesenchymal Transition in Tumor Metastasis. Annu. Rev. Pathol. Mech. Dis., 13(1), 395-412. Retrieved from https:// doi.org/10.1146/annurev-pathol-020117-043854 doi: 10.1146/annurev -pathol-020117-043854

Miyamoto, T., Akutsu, S. N., Fukumitsu, A., Morino, H., Masatsuna, Y., Hosoba, K., ... Matsuura, S. (2017, nov). PLK1-mediated phosphorylation of WDR62/MCPH2 ensures proper mitotic spindle orientation. Hum. Mol. Genet., 26 (22), 4429-4440. Retrieved from http://www.ncbi.nlm.nih.gov/pubmed/ 28973348 doi: 10.1093/hmg/ddx330

Miyamoto, T., Hosoba, K., Ochiai, H., Royba, E., Izumi, H., Sakuma, T., ... Matsuura, S. (2015, feb). The Microtubule-Depolymerizing Activity of a Mitotic Kinesin Protein KIF2A Drives Primary Cilia Disassembly Coupled with Cell Proliferation. Cell Rep., 10(5), 664-673. Retrieved from http:// www.ncbi.nlm.nih.gov/pubmed/25660017 doi: 10.1016/j.celrep.2015.01.003

Moran, E. (1993, feb). DNA tumor virus transforming proteins and the cell cycle. Curr. Opin. Genet. Dev., 3(1), 63-70. Retrieved from http://www.ncbi.nlm .nih.gov/pubmed/8453277

Möröy, T., \& Geisen, C. (2004, aug). Cyclin E. Int. J. Biochem. Cell Biol., 36(8), 1424-39. Retrieved from https://linkinghub.elsevier.com/retrieve/ pii/S1357272503004321 doi: 10.1016/j.biocel.2003.12.005

Mortensen, L. S., Schmidt, H., Farsi, Z., Barrantes-Freer, A., Rubio, M. E., Ufartes, R., ... Pardo, L. A. (2015, jan). KV 10.1 opposes activity-dependent increase in $\mathrm{Ca}^{2+}$ influx into the presynaptic terminal of the parallel fibre-Purkinje cell synapse. J. Physiol., 593(1), 181-96. Retrieved from http://doi.wiley.com/ 10.1113/jphysiol.2014.281600 doi: 10.1113/jphysiol.2014.281600

Moyle, M. W., Kim, T., Hattersley, N., Espeut, J., Cheerambathur, D. K., Oegema, K., \& Desai, A. (2014, mar). A Bub1-Mad1 interaction targets the Mad1-Mad2 complex to unattached kinetochores to initiate the spindle checkpoint. $J$. Cell Biol., 204(5), 647-657. Retrieved from http://www.ncbi.nlm.nih.gov/ pubmed/24567362 doi: 10.1083/jcb.201311015

Müller, C., Gross, D., Sarli, V., Gartner, M., Giannis, A., Bernhardt, G., \& Buschauer, A. (2006, nov). Inhibitors of kinesin Eg5: antiproliferative activity of monastrol 
analogues against human glioblastoma cells. Cancer Chemother. Pharmacol., 59(2), 157-164. Retrieved from http://www.ncbi.nlm.nih.gov/pubmed/ 16703323 doi: 10.1007/s00280-006-0254-1

Murata, H., Tajima, N., Nagashima, Y., Yao, M., Baba, M., Goto, M., ... Kanno, H. (2002, dec). Von Hippel-Lindau tumor suppressor protein transforms human neuroblastoma cells into functional neuron-like cells. Cancer Res., 62(23), 7004-11. Retrieved from http://www.ncbi.nlm.nih.gov/pubmed/12460920

Musacchio, A., \& Salmon, E. D. (2007, may). The spindle-assembly checkpoint in space and time. Nat. Rev. Mol. Cell Biol., 8(5), 379-393. Retrieved from http://www.nature.com/doifinder/10.1038/nrm2163 doi: 10.1038/ nrm2163

Nam, H.-J., Naylor, R. M., \& van Deursen, J. M. (2015, feb). Centrosome dynamics as a source of chromosomal instability. Trends Cell Biol., 25(2), 65-73. Retrieved from http://www.ncbi.nlm.nih.gov/pubmed/25455111 doi: 10.1016/j.tcb .2014 .10 .002

Neri, M., Milazzo, D., Ugolini, D., Milic, M., Campolongo, A., Pasqualetti, P., \& Bonassi, S. (2015, jan). Worldwide interest in the comet assay: A bibliometric study. Mutagenesis, 30(1), 155-163. Retrieved from http://www.ncbi.nlm .nih.gov/pubmed/25527738 doi: 10.1093/mutage/geu061

Nigg, E. A., \& Stearns, T. (2011, oct). The centrosome cycle: Centriole biogenesis, duplication and inherent asymmetries. Nat. Cell Biol., 13(10), 115460. Retrieved from http://www.ncbi.nlm.nih.gov/pubmed/21968988 doi: $10.1038 /$ ncb2345

Nishida, E., Kumagai, H., Ohtsuki, I., \& Sakai, H. (1979, may). The interactions between calcium-dependent regulator protein of cyclic nucleotide phosphodiesterase and microtubule proteins. I. Effect of calcium-dependent regulator protein on the calcium sensitivity of microtubule assembly. J. Biochem., 85(5), 1257-66. Retrieved from http://www.ncbi.nlm.nih.gov/pubmed/221450

Nüsse, M., \& Egner, H. J. (1984, jan). Can nocodazole, an inhibitor of microtubule formation, be used to synchronize mammalian cells? Accumulation of cells in mitosis studied by two parametric flow cytometry using acridine orange and by DNA distribution analysis. Cell Tissue Kinet., 17(1), 13-23. Retrieved from 


\section{REFERENCES}

http://www.ncbi.nlm.nih.gov/pubmed/6692463

Nyberg, K. A., Michelson, R. J., Putnam, C. W., \& Weinert, T. A. (2002, dec). Toward Maintaining the Genome: DNA Damage and Replication Checkpoints. Annu. Rev. Genet., 36(1), 617-656. Retrieved from http://www.ncbi.nlm .nih.gov/pubmed/12429704 doi: 10.1146/annurev.genet.36.060402.113540

O'Brien, E. T., Salmon, E., \& Erickson, H. P. (1997). How calcium causes microtubule depolymerization. Cell Motil. Cytoskeleton, 36(2), 125-135. Retrieved from http://www.ncbi.nlm.nih.gov/pubmed/9015201 doi: 10.1002/(SICI)1097 -0169(1997)36:2〈125::AID-CM3〉3.0.CO;2-8

Occhiodoro, T., Bernheim, L., Liu, J. H., Bijlenga, P., Sinnreich, M., Bader, C. R., \& Fischer-Lougheed, J. (1998, aug). Cloning of a human ether-a-go-go potassium channel expressed in myoblasts at the onset of fusion. FEBS Lett., $434(1-2)$, 177-82. Retrieved from http://www.ncbi.nlm.nih.gov/pubmed/9738473

Ohtsubo, M., Theodoras, A. M., Schumacher, J., Roberts, J. M., \& Pagano, M. (1995, may). Human cyclin E, a nuclear protein essential for the G1-to-S phase transition. Mol. Cell. Biol., 15(5), 2612-24. Retrieved from http:// www.ncbi.nlm.nih.gov/pubmed/7739542

Olmsted, J. B., \& Borisy, G. G. (1975, jul). Ionic and nucleotide requirements for microtubule polymerization in vitro. Biochemistry, 14(13), 2996-3005. Retrieved from http://www.ncbi.nlm.nih.gov/pubmed/238580

Ouadid-Ahidouch, H., \& Ahidouch, A. (2013, aug). K+ channels and cell cycle progression in tumor cells. Front. Physiol., 4, 220. Retrieved from http://journal .frontiersin.org/article/10.3389/fphys.2013.00220/abstract doi: 10.3389 /fphys.2013.00220

Ouadid-Ahidouch, H., Ahidouch, A., \& Pardo, L. A. (2016, may). Kv10.1 K+ channel: from physiology to cancer. Pflügers Arch. - Eur. J. Physiol., 468(5), 751-762. Retrieved from http://www.ncbi.nlm.nih.gov/pubmed/26743871 doi: $10.1007 / \mathrm{s} 00424-015-1784-3$

Ousingsawat, J., Spitzner, M., Puntheeranurak, S., Terracciano, L., Tornillo, L., Bubendorf, L., ... Schreiber, R. (2007, feb). Expression of Voltage-Gated Potassium Channels in Human and Mouse Colonic Carcinoma. Clin. Cancer Res., 13(3), 824-831. Retrieved from http://www.ncbi.nlm.nih.gov/ 
pubmed/17289873 doi: 10.1158/1078-0432.CCR-06-1940

Papazian, D. M., Schwarz, T. L., Tempel, B. L., Jan, Y. N., \& Jan, L. Y. (1987, aug). Cloning of genomic and complementary DNA from Shaker, a putative potassium channel gene from Drosophila. Science, 237(4816), 749-53. Retrieved from http://www.ncbi.nlm.nih.gov/pubmed/2441470

Pardee, A. B. (1974, apr). A restriction point for control of normal animal cell proliferation. Proc. Natl. Acad. Sci. U. S. A., 71(4), 1286-90. Retrieved from http://www.ncbi.nlm.nih.gov/pubmed/4524638

Pardo, L. A., del Camino, D., Sánchez, A., Alves, F., Brüggemann, A., Beckh, S., \& Stühmer, W. (1999, oct). Oncogenic potential of EAG K+ channels. EMBO J., 18(20), 5540-5547. Retrieved from http://www.ncbi.nlm.nih.gov/pubmed/ 10523298 doi: 10.1093/emboj/18.20.5540

Pardo, L. A., \& Stühmer, W. (2013, dec). The roles of K+ channels in cancer. Nat. Rev. Cancer, 14(1), 39-48. Retrieved from http://www.nature.com/ doifinder/10.1038/nrc3635 doi: 10.1038/nrc3635

Parekh, A. B. (2011, feb). Decoding cytosolic Ca2+ oscillations. Trends Biochem. Sci., 36(2), 78-87. Retrieved from http://www.ncbi.nlm.nih.gov/pubmed/ 20810284 doi: 10.1016/j.tibs.2010.07.013

Parrilla, A., Cirillo, L., Thomas, Y., Gotta, M., Pintard, L., \& Santamaria, A. (2016, dec). Mitotic entry: The interplay between Cdk1, Plk1 and Bora. Cell Cycle, 15(23), 3177-3182. Retrieved from https://doi.org/10.1080/ 15384101.2016.1249544 doi: 10.1080/15384101.2016.1249544

Patil, M., Pabla, N., \& Dong, Z. (2013, nov). Checkpoint kinase 1 in DNA damage response and cell cycle regulation. Cell. Mol. Life Sci., 70(21), 400921. Retrieved from http://www.ncbi.nlm.nih.gov/pubmed/23508805 doi: $10.1007 / \mathrm{s} 00018-013-1307-3$

Pease, J. C., \& Tirnauer, J. S. (2011, apr). Mitotic spindle misorientation in cancer - out of alignment and into the fire. J. Cell Sci., 124(7), 1007 LP - 1016. Retrieved from http://jcs.biologists.org/content/124/7/1007 . abstract doi: $10.1242 /$ jes.081406

Peddibhotla, S., Lam, M. H., Gonzalez-Rimbau, M., \& Rosen, J. M. (2009, mar). The DNA-damage effector checkpoint kinase 1 is essential for chro- 


\section{REFERENCES}

mosome segregation and cytokinesis. Proc. Natl. Acad. Sci., 106(13), 51595164. Retrieved from http://www.ncbi.nlm.nih.gov/pubmed/19289837 doi: $10.1073 /$ pnas.0806671106

Perry, J. A., \& Kornbluth, S. (2007, may). Cdc25 and Wee1: analogous opposites? Cell Div., 2, 12. Retrieved from https://www.ncbi.nlm.nih.gov/pubmed/ 17480229 doi: $10.1186 / 1747-1028-2-12$

Peters, J.-M. (2006, aug). The anaphase promoting complex/cyclosome: a machine designed to destroy. Nat. Rev. Mol. Cell Biol., 7, 644. Retrieved from https://doi.org/10.1038/nrm1988

Phengchat, R., Takata, H., Morii, K., Inada, N., Murakoshi, H., Uchiyama, S., \& Fukui, K. (2016, dec). Calcium ions function as a booster of chromosome condensation. Sci. Rep., 6(1), 38281. Retrieved from http://www . nature.com/ articles/srep38281 doi: 10.1038/srep38281

Phua, S. C., Chiba, S., Suzuki, M., Su, E., Roberson, E. C., Pusapati, G. V., ... Inoue, T. (2017, jan). Dynamic Remodeling of Membrane Composition Drives Cell Cycle through Primary Cilia Excision. Cell, 168(1-2), 264-279.e15. Retrieved from http://www.ncbi.nlm.nih.gov/pubmed/28086093 doi: 10 .1016/j.cell.2016.12.032

Plotnikova, O. V., Nikonova, A. S., Loskutov, Y. V., Kozyulina, P. Y., Pugacheva, E. N., \& Golemis, E. A. (2012, jul). Calmodulin activation of Aurora-A kinase (AURKA) is required during ciliary disassembly and in mitosis. Mol. Biol. Cell, 23(14), 2658-70. Retrieved from http://www.ncbi.nlm.nih.gov/pubmed/ 22621899 doi: 10.1091/mbc.E11-12-1056

Plotnikova, O. V., Pugacheva, E. N., Dunbrack, R. L., \& Golemis, E. A. (2010, sep). Rapid calcium-dependent activation of Aurora-A kinase. Nat. Commun., 1(6), 18. Retrieved from http://www . nature.com/doifinder/10.1038/ncomms1061 doi: $10.1038 /$ ncomms1061

Plotnikova, O. V., Pugacheva, E. N., \& Golemis, E. A. (2009). Primary cilia and the cell cycle. Methods Cell Biol., 94, 137-60. Retrieved from http://www.ncbi .nlm.nih.gov/pubmed/20362089 doi: 10.1016/S0091-679X(08)94007-3

Poenie, M., Alderton, J., Steinhardt, R., \& Tsien, R. (1986, aug). Calcium rises abruptly and briefly throughout the cell at the onset of anaphase. Science, 
233(4766), 886-9. Retrieved from http://www.ncbi.nlm.nih.gov/pubmed/ 3755550

Poon, R. Y. C. (2016). Cell Cycle Control: A System of Interlinking Oscillators. In Methods mol. biol. (Vol. 1342, pp. 3-19). Retrieved from http://www.ncbi .nlm.nih.gov/pubmed/26254915 doi: 10.1007/978-1-4939-2957-3_1

Prinz, S., Hwang, E. S., Visintin, R., \& Amon, A. (1998, jun). The regulation of Cdc20 proteolysis reveals a role for APC components Cdc23 and Cdc27 during S phase and early mitosis. Curr. Biol., 8(13), 750-60. Retrieved from http://www.ncbi.nlm.nih.gov/pubmed/9651679

Pugacheva, E. N., Jablonski, S. A., Hartman, T. R., Henske, E. P., \& Golemis, E. A. (2007, jun). HEF1-Dependent Aurora A Activation Induces Disassembly of the Primary Cilium. Cell, 129(7), 1351-1363. Retrieved from http://ac.els-cdn.com/S0092867407005466/1-s2.0-S0092867407005466 -main .pdf?\{_\}tid=8b10bda2-7870-11e7-bff7-00000aab0f6c $\{\&\} a c d n a t=$ 1501781222\{_\}bd21ee15a07fd7df966137c8ca2d0e52 doi: 10.1016/ j.cell.2007.04.035

Qin, T., Li, J., Yuan, M., \& Mao, T. (2012, jul). Characterization of the role of calcium in regulating the microtubule-destabilizing activity of MDP25. Plant Signal. Behav., 7(7), 708-10. Retrieved from http://www.ncbi.nlm.nih.gov/ pubmed/22751329 doi: 10.4161/psb.20336

Ramos Gomes, F., Romaniello, V., Sánchez, A., Weber, C., Narayanan, P., Psol, M., \& Pardo, L. A. (2015, dec). Alternatively Spliced Isoforms of KV10.1 Potassium Channels Modulate Channel Properties and Can Activate Cyclin-dependent Kinase in Xenopus Oocytes. J. Biol. Chem., $290(51), 30351-65$. Retrieved from http://www.ncbi.nlm.nih.gov/pubmed/26518875 doi: 10.1074/jbc.M115 .668749

Ratan, R. R., Maxfield, F. R., \& Shelanski, M. L. (1988, sep). Long-lasting and rapid calcium changes during mitosis. J. Cell Biol., 107(3), 993-9. Retrieved from http://www.ncbi.nlm.nih.gov/pubmed/3417787

Reed, S. I. (1997). Control of the G1/S transition. Cancer Surv., 29, 7-23. Retrieved from http://www.ncbi.nlm.nih.gov/pubmed/9338094

Reed, S. I. (2006). The ubiquitin-proteasome pathway in cell cycle control. Results 


\section{REFERENCES}

Probl. Cell Differ., 42, 147-81. Retrieved from http://www.ncbi.nlm.nih .gov/pubmed/16903211

Reichert, M., Bakir, B., Moreira, L., Pitarresi, J. R., Feldmann, K., Simon, L., ... Rustgi, A. K. (2018, jun). Regulation of Epithelial Plasticity Determines Metastatic Organotropism in Pancreatic Cancer. Dev. Cell, 45(6), 696711.e8. Retrieved from https://www.sciencedirect.com/science/article/ pii/S1534580718304180 doi: 10.1016/J.DEVCEL.2018.05.025

Reiter, J. F., \& Leroux, M. R. (2017, jul). Genes and molecular pathways underpinning ciliopathies. Nat. Rev. Mol. Cell Biol., 18(9), 533-547. Retrieved from http://www.ncbi.nlm.nih.gov/pubmed/28698599 doi: 10.1038/nrm.2017 .60

Renwick, A. G. (1999, jul). The metabolism of antihistamines and drug interactions: the role of cytochrome P450 enzymes. Clin. Exp. Allergy, 29 Suppl 3, 116-24. Retrieved from http://www.ncbi.nlm.nih.gov/pubmed/10444225

Rettig, J., Heinemann, S. H., Wunder, F., Lorra, C., Parcej, D. N., Dolly, J. O., \& Pongs, O. (1994, may). Inactivation properties of voltage-gated $\mathrm{K}+$ channels altered by presence of beta-subunit. Nature, 369(6478), 289-94. Retrieved from http://www.nature.com/articles/369289a0 doi: 10.1038/369289a0

Rock, K. L., Gramm, C., Rothstein, L., Clark, K., Stein, R., Dick, L., ... Goldberg, A. L. (1994, sep). Inhibitors of the proteasome block the degradation of most cell proteins and the generation of peptides presented on MHC class I molecules. Cell, 78(5), 761-71. Retrieved from http://www.ncbi.nlm.nih.gov/pubmed/ 8087844

Roderick, H. L., \& Cook, S. J. (2008, may). Ca2+ signalling checkpoints in cancer: remodelling $\mathrm{Ca} 2+$ for cancer cell proliferation and survival. Nat. Rev. Cancer, 8(5), 361-375. Retrieved from http://www.ncbi.nlm.nih.gov/ pubmed/18432251 doi: 10.1038/nrc2374

Ronca, R., Benkheil, M., Mitola, S., Struyf, S., \& Liekens, S. (2017, nov). Tumor angiogenesis revisited: Regulators and clinical implications. Med. Res. Rev., 37(6), 1231-1274. Retrieved from http://www.ncbi.nlm.nih.gov/pubmed/ 28643862 doi: 10.1002/med.21452

Roshak, A. K., Capper, E. A., Imburgia, C., Fornwald, J., Scott, G., \& Marshall, L. A. 
(2000). The human polo-like kinase, PLK, regulates cdc2/cyclin B through phosphorylation and activation of the cdc25C phosphatase. Cell. Signal., 12(6), 405-411.

Rueden, C. T., Schindelin, J., Hiner, M. C., DeZonia, B. E., Walter, A. E., Arena, E. T., \& Eliceiri, K. W. (2017, dec). ImageJ2: ImageJ for the next generation of scientific image data. BMC Bioinformatics, 18(1), 529. Retrieved from http:// WwW.ncbi.nlm.nih.gov/pubmed/29187165 doi: 10.1186/s12859-017-1934-Z

Russa, A. D., Ishikita, N., Masu, K., Akutsu, H., Saino, T., \& Satoh, Y.-i. (2008, dec). Microtubule remodeling mediates the inhibition of store-operated calcium entry (SOCE) during mitosis in COS-7 cells. Arch. Histol. Cytol., 71(4), 249-63. Retrieved from http://www.ncbi.nlm.nih.gov/pubmed/19359807

Sadaghiani, A. M., Lee, S. M., Odegaard, J. I., Leveson-Gower, D. B., McPherson, O. M., Novick, P., .. Park, C. Y. (2014, oct). Identification of Orai1 Channel Inhibitors by Using Minimal Functional Domains to Screen Small Molecule Microarrays. Chem. Biol., 21(10), 1278-1292. Retrieved from http://www .ncbi.nlm.nih.gov/pubmed/25308275 doi: 10.1016/j.chembiol.2014.08.016

Safinya, C. R., Chung, P. J., Song, C., Li, Y., Ewert, K. K., \& Choi, M. C. (2016, jun). The effect of multivalent cations and Tau on paclitaxel-stabilized microtubule assembly, disassembly, and structure. Adv. Colloid Interface Sci., 232, 916. Retrieved from http://www.ncbi.nlm.nih.gov/pubmed/26684364 doi: 10.1016/j.cis.2015.11.002

Samanta, K., \& Parekh, A. B. (2017, may). Spatial Ca2+ profiling: decrypting the universal cytosolic Ca2+ oscillation. J. Physiol., 595(10), 3053-3062. Retrieved from http://doi.wiley.com/10.1113/JP272860 doi: 10.1113/JP272860

Sana, S., Keshri, R., Rajeevan, A., Kapoor, S., \& Kotak, S. (2018, dec). Plk1 regulates spindle orientation by phosphorylating NuMA in human cells. Life Sci. Alliance, 1(6), e201800223. Retrieved from http://www.ncbi.nlm.nih.gov/pubmed/ 30456393 doi: 10.26508/lsa.201800223

Sánchez, A., Urrego, D., \& Pardo, L. A. (2016a, may). Cyclic expression of the voltagegated potassium channel $\mathrm{K}<\mathrm{sub}>\mathrm{V}</ \mathrm{sub}>10.1$ promotes disassembly of the primary cilium. EMBO Rep., 17(5), 708-723. Retrieved from http://embor .embopress.org/lookup/doi/10.15252/embr.201541082 doi: 10.15252/ 


\section{REFERENCES}

embr.201541082

Sánchez, A., Urrego, D., \& Pardo, L. A. (2016b). Cyclic expression of the voltagegated potassium channel KV10.1 promotes disassembly of the primary cilium. EMBO Rep., 17(5), 708-23. Retrieved from http://www.ncbi.nlm.nih.gov/ pubmed/27113750 doi: 10.15252/embr.201541082

Sánchez, I., \& Dynlacht, B. D. (2016, jun). Cilium assembly and disassembly. Nat. Cell Biol., 18, 711. Retrieved from https://doi.org/10.1038/ncb3370

Sandoval, I. V., \& Weber, K. (1978). Calcium-Induced Inactivation of Microtubule Formation in Brain Extracts Presence of a Calcium-Dependent Protease Acting on Polymerization-Stimulating Microtubule-Associated Proteins. Eur. J. Biochem, 92, 463-470. Retrieved from https://onlinelibrary.wiley.com/ doi/pdf/10.1111/j.1432-1033.1978.tb12768.x

Santella, L. (1998, mar). The Role of Calcium in the Cell Cycle: Facts and Hypotheses. Biochem. Biophys. Res. Commun., 244(2), 317-324. Retrieved from http:// WwW.ncbi.nlm.nih.gov/pubmed/9514855 doi: 10.1006/bbrc.1998.8086

Sataric, M. V., Sekulic, D. L., Sataric, B. M., \& Zdravkovic, S. (2015, nov). Role of nonlinear localized Ca $2+$ pulses along microtubules in tuning the mechano-sensitivity of hair cells. Prog. Biophys. Mol. Biol., 119(2), 162174. Retrieved from http://www.ncbi.nlm.nih.gov/pubmed/26208473 doi: 10.1016/j.pbiomolbio.2015.07.009

Saurin, A. T. (2018). Kinase and Phosphatase Cross-Talk at the Kinetochore. Front. cell Dev. Biol., 6, 62. Retrieved from http://www.ncbi.nlm.nih.gov/ pubmed/29971233 doi: 10.3389/fcell.2018.00062

Scheffner, M., Werness, B. A., Huibregtse, J. M., Levine, A. J., \& Howley, P. M. (1990, dec). The E6 oncoprotein encoded by human papillomavirus types 16 and 18 promotes the degradation of p53. Cell, 63(6), 1129-36. Retrieved from http://www.ncbi.nlm.nih.gov/pubmed/2175676

Schlaitz, A.-L. (2014, jul). Microtubules as key coordinators of nuclear envelope and endoplasmic reticulum dynamics during mitosis. Bioessays, 36(7), 66571. Retrieved from http://doi.wiley.com/10.1002/bies.201400022 doi: 10.1002/bies.201400022

Schmidt, M., Rohe, A., Platzer, C., Najjar, A., Erdmann, F., \& Sippl, W. (2017, nov). 
Regulation of G2/M Transition by Inhibition of WEE1 and PKMYT1 Kinases. Molecules, 22(12), 2045. Retrieved from http://www.ncbi.nlm.nih.gov/ pubmed/29168755 doi: 10.3390/molecules22122045

Schneider, C. A., Rasband, W. S., \& Eliceiri, K. W. (2012, jul). NIH Image to ImageJ: 25 years of image analysis. Nat. Methods, 9(7), 671-5. Retrieved from http://www.ncbi.nlm.nih.gov/pubmed/22930834

Schonherr, R., Löber, K., \& Heinemann, S. H. (2000, jul). Inhibition of human ether a go-go potassium channels by Ca2+/calmodulin. EMBO J., 19(13), 32633271. Retrieved from http://www.ncbi.nlm.nih.gov/pubmed/10880439 doi: $10.1093 / \mathrm{emboj} / 19.13 .3263$

Schwab, A., Fabian, A., Hanley, P. J., \& Stock, C. (2012, oct). Role of Ion Channels and Transporters in Cell Migration. Physiol. Rev., 92(4), 18651913. Retrieved from http://www.ncbi.nlm.nih.gov/pubmed/23073633 doi: 10.1152/physrev.00018.2011

Sclafani, R. A., \& Holzen, T. M. (2007). Cell cycle regulation of DNA replication. Annu. Rev. Genet., 41, 237-80. Retrieved from http://www.ncbi.nlm.nih .gov/pubmed/17630848 doi: 10.1146/annurev.genet.41.110306.130308

Seki, A., Coppinger, J. A., Jang, C.-Y., Yates, J. R., \& Fang, G. (2008). Bora and the kinase Aurora a cooperatively activate the kinase Plk1 and control mitotic entry. Science (80-. )., 320(5883), 1655-1658.

Semenza, G. L. (2013, aug). Cancer-stromal cell interactions mediated by hypoxiainducible factors promote angiogenesis, lymphangiogenesis, and metastasis. Oncogene, 32(35), 4057-63. Retrieved from http://www.nature.com/ articles/onc2012578 doi: 10.1038/onc.2012.578

Shaltiel, I. A., Krenning, L., Bruinsma, W., \& Medema, R. H. (2015, feb). The same, only different - DNA damage checkpoints and their reversal throughout the cell cycle. J. Cell Sci., 128(4), 607-20. Retrieved from http://www.ncbi.nlm .nih.gov/pubmed/25609713 doi: 10.1242/jcs.163766

Shandilya, J., Medler, K. F., \& Roberts, S. G. E. (2016, aug). Regulation of AURORA B function by mitotic checkpoint protein MAD2. Cell Cycle, 15(16), 21962201. Retrieved from http://www.ncbi.nlm.nih.gov/pubmed/27341405 doi: 10.1080/15384101.2016.1200773 


\section{REFERENCES}

Shang, Z., Yu, L., Lin, Y.-F., Matsunaga, S., Shen, C.-Y., \& Chen, B. P. C. (2014, feb). DNA-PKcs activates the Chk2-Brca1 pathway during mitosis to ensure chromosomal stability. Oncogenesis, 3(2), e85. Retrieved from http:// www.ncbi.nlm.nih.gov/pubmed/24492479 doi: 10.1038/oncsis.2013.49

Shao, H., Huang, Y., Zhang, L., Yuan, K., Chu, Y., Dou, Z., .. Yao, X. (2015, dec). Spatiotemporal dynamics of Aurora B-PLK1-MCAK signaling axis orchestrates kinetochore bi-orientation and faithful chromosome segregation. Sci. Rep., 5(1), 12204. Retrieved from http://www.ncbi.nlm.nih.gov/pubmed/26206521 doi: $10.1038 /$ srep12204

Sherwood, L. M., Parris, E. E., \& Folkman, J. (1971, nov). Tumor Angiogenesis: Therapeutic Implications. N. Engl. J. Med., 285(21), 1182-1186. Retrieved from http://www.ncbi.nlm.nih.gov/pubmed/4938153 doi: 10.1056/ NEJM197111182852108

Sierra, L. M., \& Gaivão, I. (Eds.). (2014). Genotoxicity and DNA Repair. New York, NY: Springer New York. Retrieved from http://link.springer.com/ 10.1007/978-1-4939-1068-7 doi: 10.1007/978-1-4939-1068-7

Singh, N. P., McCoy, M. T., Tice, R. R., \& Schneider, E. L. (1988, mar). A simple technique for quantitation of low levels of DNA damage in individual cells. Exp. Cell Res., 175(1), 184-91. Retrieved from http://www.ncbi.nlm.nih.gov/ pubmed/3345800

Skoufias, D. A., Andreassen, P. R., Lacroix, F. B., Wilson, L., \& Margolis, R. L. (2001, apr). Mammalian mad2 and bub1/bubR1 recognize distinct spindleattachment and kinetochore-tension checkpoints. Proc. Natl. Acad. Sci. U. S. A., 98(8), 4492-7. Retrieved from http://www.pnas.org/cgi/doi/10.1073/ pnas.081076898 doi: 10.1073/pnas.081076898

Smedler, E., \& Uhlén, P. (2014, mar). Frequency decoding of calcium oscillations. Biochim. Biophys. Acta - Gen. Subj., 1840(3), 964-969. Retrieved from http:// www.ncbi.nlm.nih.gov/pubmed/24269537 doi: 10.1016/j.bbagen.2013.11 .015

Smith, J., Mun Tho, L., Xu, N., \& A. Gillespie, D. (2010, jan). The ATM-Chk2 and ATR-Chk1 Pathways in DNA Damage Signaling and Cancer. Adv. Cancer Res., 108, 73-112. Retrieved from https://www.sciencedirect.com/science/ 


\section{REFERENCES}

article/pii/B9780123808882000030?via\{\%\}3Dihub doi: 10.1016/B978-0 $-12-380888-2.00003-0$

Smith, P. K., Krohn, R. I., Hermanson, G. T., Mallia, A. K., Gartner, F. H., Provenzano, M. D., .. Klenk, D. C. (1985, oct). Measurement of protein using bicinchoninic acid. Anal. Biochem., 150(1), 76-85. Retrieved from http://www.ncbi.nlm.nih.gov/pubmed/3843705

Smits, V. A. J., Klompmaker, R., Arnaud, L., Rijksen, G., Nigg, E. A., \& Medema, R. H. (2000, sep). Polo-like kinase-1 is a target of the DNA damage checkpoint. Nat. Cell Biol., 2(9), 672-676. Retrieved from http://www.ncbi.nlm.nih .gov/pubmed/10980711 doi: 10.1038/35023629

Söderberg, O., Gullberg, M., Jarvius, M., Ridderstråle, K., Leuchowius, K.-J., Jarvius, J., ... Landegren, U. (2006, dec). Direct observation of individual endogenous protein complexes in situ by proximity ligation. Nat. Methods, 3(12), 9951000. Retrieved from http://www.ncbi.nlm.nih.gov/pubmed/17072308 doi: 10.1038/nmeth947

Sørensen, C. S., Syljuåsen, R. G., Falck, J., Schroeder, T., Rönnstrand, L., Khanna, K. K., .. Lukas, J. (2003, mar). Chk1 regulates the S phase checkpoint by coupling the physiological turnover and ionizing radiation-induced accelerated proteolysis of Cdc25A. Cancer Cell, 3(3), 247-58. Retrieved from http:// www.ncbi.nlm.nih.gov/pubmed/12676583

Speit, G., \& Rothfuss, A. (2012). The comet assay: a sensitive genotoxicity test for the detection of DNA damage and repair. Methods Mol. Biol., 920, 7990. Retrieved from http://link.springer.com/10.1007/978-1-61779-998 -3\{_\}6 doi: 10.1007/978-1-61779-998-3_6

Spitzner, M., Martins, J. R., Soria, R. B., Ousingsawat, J., Scheidt, K., Schreiber, R., \& Kunzelmann, K. (2008, mar). Eag1 and Bestrophin 1 Are Up-regulated in Fast-growing Colonic Cancer Cells. J. Biol. Chem., 283(12), 7421-7428. Retrieved from http://www.ncbi.nlm.nih.gov/pubmed/18222922 doi: 10 $.1074 /$ jbc.M703758200

Spitzner, M., Ousingsawat, J., Scheidt, K., Kunzelmann, K., \& Schreiber, R. (2006, nov). Voltage-gated $\mathrm{K}+$ channels support proliferation of colonic carcinoma cells. FASEB J., 21(1), 35-44. Retrieved from http://www.ncbi.nlm.nih.gov/ 


\section{REFERENCES}

pubmed/17135369 doi: 10.1096/fj.06-6200com

Spring, K.R., J.C. Russ, M.J. Parry-Hill, T.J. Fellers, M. D. (2016). Difference of Gaussians Edge Enhancement Algorithm - Interactive Tutorial. Molecular Expressions Microscopy Primer: Digital Image Processing. Retrieved 2019-0303, from https://micro.magnet.fsu.edu/primer/java/digitalimaging/ processing/diffgaussians/index.html

Stansfeld, C. E., Röper, J., Ludwig, J., Weseloh, R. M., Marsh, S. J., Brown, D. A., \& Pongs, O. (1996, sep). Elevation of intracellular calcium by muscarinic receptor activation induces a block of voltage-activated rat ether-à-go-go channels in a stably transfected cell line. Proc. Natl. Acad. Sci. U. S. A., 93(18), 9910-4. Retrieved from http://www.ncbi.nlm.nih.gov/pubmed/8790430

Stevens, L., Ju, M., \& Wray, D. (2009, apr). Roles of surface residues of intracellular domains of heag potassium channels. Eur. Biophys. J., 38(4), 523-32. Retrieved from http://link. springer.com/10.1007/s00249-009-0402-8 doi: $10.1007 / \mathrm{s} 00249-009-0402-8$

Stolz, A., Ertych, N., \& Bastians, H. (2015). Microtubule plus tips: A dynamic route to chromosomal instability. Mol. Cell. Oncol., 2(2), e960768. Retrieved from http://www.ncbi.nlm.nih.gov/pubmed/27308411 doi: 10.4161/23723548 .2014 .960768

Stolz, A., Ertych, N., Kienitz, A., Vogel, C., Schneider, V., Fritz, B., .. Bastians, H. (2010, may). The CHK2-BRCA1 tumour suppressor pathway ensures chromosomal stability in human somatic cells. Nat. Cell Biol., 12(5), 492499. Retrieved from http://www.ncbi.nlm.nih.gov/pubmed/20364141 doi: $10.1038 /$ ncb2051

Stühmer, W., Conti, F., Suzuki, H., Wang, X. D., Noda, M., Yahagi, N., .. Numa, S. (1989, jun). Structural parts involved in activation and inactivation of the sodium channel. Nature, 339 (6226), 597-603. Retrieved from http:// Www.nature.com/articles/339597a0 doi: 10.1038/339597a0

Stumpff, J., von Dassow, G., Wagenbach, M., Asbury, C., \& Wordeman, L. (2008). The Kinesin-8 Motor Kif18A Suppresses Kinetochore Movements to Control Mitotic Chromosome Alignment. Dev. Cell, 14 (2), 252262. Retrieved from http://www.sciencedirect.com/science/article/ 
pii/S1534580707004364 doi: https://doi.org/10.1016/j.devcel.2007.11.014

Stumpff, J., Wagenbach, M., Franck, A., Asbury, C. L., \& Wordeman, L. (2012). Kif18A and Chromokinesins Confine Centromere Movements via Microtubule Growth Suppression and Spatial Control of Kinetochore Tension. Dev. Cell, 22(5), 1017-1029. Retrieved from http://www.sciencedirect.com/science/ article/pii/S1534580712000949 doi: https://doi.org/10.1016/j.devcel.2012 .02 .013

Su, X., Qiu, W., Gupta, M. L., Pereira-Leal, J. B., Reck-Peterson, S. L., Pellman, D., \& Pellman, D. (2011, sep). Mechanisms underlying the dual-mode regulation of microtubule dynamics by Kip3/kinesin-8. Mol. Cell, 43(5), 75163. Retrieved from http://www.ncbi.nlm.nih.gov/pubmed/21884976 doi: 10.1016/j.molcel.2011.06.027

Subramanian, R., \& Kapoor, T. M. (2013, nov). Slipping past the spindle assembly checkpoint. Nat. Cell Biol., 15(11), 1261-1263. Retrieved from http:// Www.ncbi.nlm.nih.gov/pubmed/24189515 doi: 10.1038/ncb2876

Subramanyam, P., \& Colecraft, H. M. (2015, jan). Ion Channel Engineering: Perspectives and Strategies. J. Mol. Biol., 427(1), 190-204. Retrieved from http://www.ncbi.nlm.nih.gov/pubmed/25205552 doi: 10.1016/j.jmb.2014 .09 .001

Sugden, M. C., Christie, M. R., \& Ashcroft, S. J. H. (1979). Presence and possible role of calcium-dependent regulator (calmodulin) in rat islets of langerhans. FEBS Lett., 105(1), 95-100. Retrieved from http: //www . sciencedirect.com/ science/article/pii/0014579379808947 doi: https://doi.org/10.1016/ 0014-5793(79)80894-7

Sun, X. X., Hodge, J. J. L., Zhou, Y., Nguyen, M., \& Griffith, L. C. (2004, mar). The eag potassium channel binds and locally activates calcium/calmodulindependent protein kinase II. J. Biol. Chem., 279(11), 10206-14. Retrieved from http://www.jbc.org/lookup/doi/10.1074/jbc.M310728200 doi: 10.1074/ jbc.M310728200

Swartz, K. J. (2004, dec). Towards a structural view of gating in potassium channels. Nat. Rev. Neurosci., 5(12), 905-16. Retrieved from http://www.nature.com/ doifinder/10.1038/nrn1559 doi: 10.1038/nrn1559 


\section{REFERENCES}

Szabó, I., Bock, J., Grassmé, H., Soddemann, M., Wilker, B., Lang, F., .. Gulbins, E. (2008, sep). Mitochondrial potassium channel Kv1.3 mediates Bax-induced apoptosis in lymphocytes. Proc. Natl. Acad. Sci. U. S. A., 105(39), 148616. Retrieved from http://www.ncbi.nlm.nih.gov/pubmed/18818304 doi: $10.1073 /$ pnas.0804236105

Takahashi, Y., Kitadai, Y., Bucana, C. D., Cleary, K. R., \& Ellis, L. M. (1995, sep). Expression of vascular endothelial growth factor and its receptor, KDR, correlates with vascularity, metastasis, and proliferation of human colon cancer. Cancer Res., 55(18), 3964-8. Retrieved from http://www.ncbi.nlm.nih.gov/ pubmed/7664263

Tan, M. L., Tan, H. K., \& Muhammad, T. S. T. (2015). Apoptosis and Cancer. In Rezaei N. (Ed.), Cancer immunol. (pp. 209-242). Berlin, Heidelberg: Springer Berlin Heidelberg. Retrieved from http://link. springer.com/10.1007/978 -3-662-44006-3\{_\}13 doi: 10.1007/978-3-662-44006-3_13

Tempel, B. L., Papazian, D. M., Schwarz, T. L., Jan, Y. N., \& Jan, L. Y. (1987, aug). Sequence of a probable potassium channel component encoded at Shaker locus of Drosophila. Science, 237(4816), 770-5. Retrieved from http:// www.ncbi.nlm.nih.gov/pubmed/2441471

Terlau, H., Heinemann, S. H., Stühmer, W., Pongs, O., \& Ludwig, J. (1997, aug). Amino terminal-dependent gating of the potassium channel rat eag is compensated by a mutation in the S4 segment. J. Physiol., 502 ( Pt 3, 537-43. Retrieved from http://www.ncbi.nlm.nih.gov/pubmed/9279806

The MathWorks, I. (2018). Signal Processing Toolbox ${ }^{T M}$ (Tech. Rep.). Retrieved from www.mathworks.com

Thoma, C. R., Toso, A., Gutbrodt, K. L., Reggi, S. P., Frew, I. J., Schraml, P., ... Krek, W. (2009, aug). VHL loss causes spindle misorientation and chromosome instability. Nat. Cell Biol., 11(8), 994-1001. Retrieved from http://www.ncbi.nlm.nih.gov/pubmed/19620968 doi: 10.1038/ncb1912

Thomas, Y., Cirillo, L., Panbianco, C., Martino, L., Tavernier, N., Schwager, F., ... Gotta, M. (2016, apr). Cdk1 Phosphorylates SPAT-1/Bora to Promote Plk1 Activation in C. elegans and Human Cells. Cell Rep., 15(3), 510518. Retrieved from https://www.sciencedirect.com/science/article/ 
pii/S2211124716303205?via\{\%\}3Dihub doi: 10.1016/J.CELREP.2016.03 .049

Thompson, G. J., Langlais, C., Cain, K., Conley, E. C., \& Cohen, G. M. (2001, jul). Elevated extracellular $[\mathrm{K}+]$ inhibits death-receptor- and chemical-mediated apoptosis prior to caspase activation and cytochrome c release. Biochem. J., 357(Pt 1), 137-45. Retrieved from http://www.ncbi.nlm.nih.gov/pubmed/ 11415444

Tian, C., Zhu, R., Zhu, L., Qiu, T., Cao, Z., \& Kang, T. (2014, jan). Potassium Channels: Structures, Diseases, and Modulators. Chem. Biol. Drug Des., 83(1), 1-26. Retrieved from http://www.ncbi.nlm.nih.gov/pubmed/24119115 doi: $10.1111 /$ cbdd .12237

Tolić-Nørrelykke, I. M. (2008, sep). Push-me-pull-you: how microtubules organize the cell interior. Eur. Biophys. J., 37(7), 1271-1278. Retrieved from https:// www.ncbi.nlm.nih.gov/pubmed/18404264 doi: 10.1007/s00249-008-0321-0

Tombes, R. M., \& Borisy, G. G. (1989, aug). Intracellular free calcium and mitosis in mammalian cells: anaphase onset is calcium modulated, but is not triggered by a brief transient. J. Cell Biol., 109(2), 627-36. Retrieved from http:// www.ncbi.nlm.nih.gov/pubmed/2668300 doi: 10.1083/JCB.109.2.627

Tomczak, A. P., Fernández-Trillo, J., Bharill, S., Papp, F., Panyi, G., Stühmer, W., ... Pardo, L. A. (2017, may). A new mechanism of voltage-dependent gating exposed by KV10.1 channels interrupted between voltage sensor and pore. $J$. Gen. Physiol., 149(5), 577-593. Retrieved from http://www.ncbi.nlm.nih .gov/pubmed/28360219 doi: 10.1085/jgp.201611742

Ufartes, R., Schneider, T., Mortensen, L. S., de Juan Romero, C., Hentrich, K., Knoetgen, H., ... Stuehmer, W. (2013, jun). Behavioural and functional characterization of Kv10.1 (Eag1) knockout mice. Hum. Mol. Genet., 22(11), 2247-62. Retrieved from https://academic.oup.com/hmg/article-lookup/ doi/10.1093/hmg/ddt076 doi: 10.1093/hmg/ddt076

Uhlen, P. (2004, nov). Spectral Analysis of Calcium Oscillations. Sci. Signal., 2004(258), pl15-pl15. Retrieved from http://www.ncbi.nlm.nih.gov/ pubmed/15536176 doi: 10.1126/stke.2582004pl15

Urrego, D., Movsisyan, N., Ufartes, R., \& Pardo, L. (2016). Periodic expression of 


\section{REFERENCES}

Kv10.1 driven by pRb/E2F1 contributes to G2/M progression of cancer and non-transformed cells. Cell Cycle, 15(6). doi: 10.1080/15384101.2016.1138187

Urrego, D., Sánchez, A., Tomczak, A. P., \& Pardo, L. A. (2017). The electric fence to cell-cycle progression: Do local changes in membrane potential facilitate disassembly of the primary cilium? BioEssays, 39(6), 1600190. Retrieved from https://onlinelibrary.wiley.com/doi/abs/10 .1002/bies. 201600190 doi: 10.1002/bies.201600190

Urrego-blanco, D. E. (2014). Dynamics of Kv10 . 1 expression through the cell cycle of cancer and non-cancer cells (Doctoral dissertation). Retrieved from https:// ediss . uni-goettingen. de/handle/11858/00-1735-0000-0022-5FDA-E

Van Horn, R. D., Chu, S., Fan, L., Yin, T., Du, J., Beckmann, R., .. Ye, X. S. (2010, jul). Cdk1 activity is required for mitotic activation of aurora A during G2/M transition of human cells. J. Biol. Chem., 285(28), 21849-57. Retrieved from http://www.ncbi.nlm.nih.gov/pubmed/20444701 doi: 10.1074/jbc.M110 .141010

van den Heuvel, S. (2005, sep). Cell-cycle regulation. WormBook, 1-16. Retrieved from http://www.ncbi.nlm.nih.gov/pubmed/18050422 doi: 10 $.1895 /$ wormbook.1.28.1

Varshney, N., Kebede, A. A., Owusu-Dapaah, H., Lather, J., Kaushik, M., \& Bhullar, J. S. (2017, aug). A Review of Von Hippel-Lindau Syndrome. J. Kidney Cancer VHL, 4(3), 20. Retrieved from http://www.ncbi.nlm.nih .gov/pubmed/28785532 doi: 10.15586/jkcvhl.2017.88

Vater, W., Böhm, K. J., \& Unger, E. (1997). Tubulin assembly in the presence of calcium ions and taxol: Microtubule bundling and formation of macrotubule - ring complexes. Cell Motil. Cytoskeleton, 36(1), 76-83. Retrieved from http://www.ncbi.nlm.nih.gov/pubmed/8986379 doi: 10.1002/ (SICI)1097-0169(1997)36:1〈76::AID-CM7〉3.0.CO;2-F

Vauzour, D., Vafeiadou, K., Rice-Evans, C., Cadenas, E., \& Spencer, J. P. (2007, dec). Inhibition of cellular proliferation by the genistein metabolite 5,7,3',4'tetrahydroxyisoflavone is mediated by DNA damage and activation of the ATR signalling pathway. Arch. Biochem. Biophys., 468(2), 159-166. Retrieved from http://www.ncbi.nlm.nih.gov/pubmed/17976513 doi: 10.1016/j.abb.2007 
.09 .021

Vigneron, S., Sundermann, L., Labbé, J.-C., Pintard, L., Radulescu, O., Castro, A., \& Lorca, T. (2018, jun). Cyclin A-cdk1-Dependent Phosphorylation of Bora Is the Triggering Factor Promoting Mitotic Entry. Dev. Cell, 45(5), 637650.e7. Retrieved from https://doi.org/10.1016/j.devcel.2018.05.005 doi: $10.1016 /$ j.devcel.2018.05.005

Visconti, R., Palazzo, L., Della Monica, R., \& Grieco, D. (2012, jan). Fcp1-dependent dephosphorylation is required for M-phase-promoting factor inactivation at mitosis exit. Nat. Commun., 3(1), 894. Retrieved from http://www.ncbi.nlm .nih.gov/pubmed/22692537 doi: 10.1038/ncomms1886

von Schubert, C., Cubizolles, F., Bracher, J. M., Sliedrecht, T., Kops, G. J., \& Nigg, E. A. (2015, jul). Plk1 and Mps1 Cooperatively Regulate the Spindle Assembly Checkpoint in Human Cells. Cell Rep., 12(1), 66-78. Retrieved from https:// linkinghub.elsevier.com/retrieve/pii/S2211124715005902 doi: 10 $.1016 /$ j.celrep.2015.06.007

Voß, C. (2007). Production of plasmid DNA for pharmaceutical use. In Biotechnol. annu. rev. (Vol. 13, pp. 201-222). Retrieved from http://www.ncbi.nlm.nih .gov/pubmed/17875478 doi: 10.1016/S1387-2656(07)13008-8

Wadhwa, S., Wadhwa, P., Dinda, A. K., \& Gupta, N. P. (2009, jun). Differential expression of potassium ion channels in human renal cell carcinoma. Int. Urol. Nephrol., 41(2), 251-7. Retrieved from http://link.springer.com/ 10.1007/s11255-008-9459-z doi: 10.1007/s11255-008-9459-z

Walczak, C. E., Gayek, S., \& Ohi, R. (2013, oct). Microtubule-Depolymerizing Kinesins. Annu. Rev. Cell Dev. Biol., 29(1), 417-441. Retrieved from http://www.ncbi.nlm.nih.gov/pubmed/23875646 doi: 10.1146/annurev -cellbio-101512-122345

Walker, J. M. (1994). The Bicinchoninic Acid (BCA) Assay for Protein Quantitation. In Basic protein pept. protoc. (Vol. 32, pp. 5-8). New Jersey: Humana Press. Retrieved from http://www.ncbi.nlm.nih.gov/pubmed/7951748 doi: 10 $.1385 / 0-89603-268-\mathrm{X}: 5$

Wang, C., Li, Z., Fu, M., Bouras, T., \& Pestell, R. G. (2004). Signal transduction mediated by cyclin D1: from mitogens to cell proliferation: a molecular target 


\section{REFERENCES}

with therapeutic potential. Cancer Treat. Res., 119, 217-37. Retrieved from http://www.ncbi.nlm.nih.gov/pubmed/15164880

Wang, G., Chen, Q., Zhang, X., Zhang, B., Zhuo, X., Liu, J., ... Zhang, C. (2013, mar). PCM1 recruits Plk1 to the pericentriolar matrix to promote primary cilia disassembly before mitotic entry. J. Cell Sci., 126(6), 13551365. Retrieved from http://www.ncbi.nlm.nih.gov/pubmed/23345402 doi: $10.1242 /$ jcs. 114918

Wang, L.-h., Yan, M., Xu, D.-z., Cao, J.-x., Zhu, X.-F., Zeng, Y.-X., \& Liu, Q. (2008, apr). Requirement of Aurora-A kinase in astral microtubule polymerization and spindle microtubule flux. Cell Cycle, 7(8), 1104-1111. Retrieved from http:// www.tandfonline.com/doi/abs/10.4161/cc.7.8.5738 doi: 10.4161/cc.7.8 .5738

Wang, X., Xiao, A. Y., Ichinose, T., \& Yu, S. P. (2000, nov). Effects of tetraethylammonium analogs on apoptosis and membrane currents in cultured cortical neurons. J. Pharmacol. Exp. Ther., 295(2), 524-30. Retrieved from http://www.ncbi.nlm.nih.gov/pubmed/11046085

Wang, Z., Wilson, G. F., \& Griffith, L. C. (2002, jul). Calcium/calmodulin-dependent protein kinase II phosphorylates and regulates the Drosophila eag potassium channel. J. Biol. Chem., 27r(27), 24022-9. Retrieved from http://www.jbc .org/lookup/doi/10.1074/jbc.M201949200 doi: 10.1074/jbc.M201949200

Warmke, J., Drysdale, R., \& Ganetzky, B. (1991, jun). A distinct potassium channel polypeptide encoded by the Drosophila eag locus. Science, 252(5012), 1560-2. Retrieved from http://www.ncbi.nlm.nih.gov/pubmed/1840699

Watanabe, N., Arai, H., Iwasaki, J.-i., Shiina, M., Ogata, K., Hunter, T., \& Osada, H. (2005). Cyclin-dependent kinase (CDK) phosphorylation destabilizes somatic Wee1 via multiple pathways. Proc. Natl. Acad. Sci., 102(33), 11663-11668.

Waters, J. C., Chen, R. H., Murray, A. W., \& Salmon, E. D. (1998, jun). Localization of Mad2 to kinetochores depends on microtubule attachment, not tension. $J$. Cell Biol., 141(5), 1181-91. Retrieved from http://www.ncbi.nlm.nih.gov/ pubmed/9606210

Weber, C., Mello de Queiroz, F., Downie, B. R., Suckow, A., Stühmer, W., \& Pardo, L. A. (2006, may). Silencing the activity and proliferative proper- 
ties of the human EagI Potassium Channel by RNA Interference. J. Biol. Chem., 281(19), 13030-7. Retrieved from http://www.jbc.org/lookup/doi/ 10.1074/jbc.M600883200 doi: 10.1074/jbc.M600883200

Wei, A., Jegla, T., \& Salkoff, L. (1996). Eight potassium channel families revealed by the C. elegans genome project. Neuropharmacology, 35(7), 805-29. Retrieved from http://www.ncbi.nlm.nih.gov/pubmed/8938713

Whicher, J. R., \& MacKinnon, R. (2016). Structure of the voltage-gated K+ channel Eag1 reveals an alternative voltage sensing mechanism. Science (80-. )., 353(6300). Retrieved from http://science.sciencemag.org/content/ $353 / 6300 / 664$

Whitaker, M., \& Larman, M. G. (2001, feb). Calcium and mitosis. Semin. Cell Dev. Biol., 12(1), 53-58. Retrieved from http://www.ncbi.nlm.nih.gov/pubmed/ 11162747 doi: 10.1006/scdb.2000.0217

Wikipedia. (n.d.). Bicinchoninic acid assay. Retrieved from https://en.wikipedia .org/wiki/Bicinchoninic\{_\}acid\{_\}assay

Wolff, J., Sackett, D. L., \& Knipling, L. (1996, oct). Cation selective promotion of tubulin polymerization by alkali metal chlorides. Protein Sci., 5(10), 20202028. Retrieved from http://www.ncbi.nlm.nih.gov/pubmed/8897602 doi: $10.1002 /$ pro. 5560051008

Wooltorton, J. R., \& Mathie, A. (1993, nov). Block of potassium currents in rat isolated sympathetic neurones by tricyclic antidepressants and structurally related compounds. Br. J. Pharmacol., 110(3), 1126-32. Retrieved from http://www.ncbi.nlm.nih.gov/pubmed/8298801

Wray, D. (2004, may). The roles of intracellular regions in the activation of voltagedependent potassium channels. Eur. Biophys. J., 33(3), 194-200. Retrieved from http://www.ncbi.nlm.nih.gov/pubmed/14608450 doi: 10.1007/s00249 $-003-0363-2$

Wray, D. (2009, mar). Intracellular regions of potassium channels: Kv2.1 and heag. Eur. Biophys. J., 38(3), 285-292. Retrieved from http://www.ncbi.nlm.nih .gov/pubmed/18607586 doi: 10.1007/s00249-008-0354-4

Wu, H.-Y., Nazockdast, E., Shelley, M. J., \& Needleman, D. J. (2017, feb). Forces positioning the mitotic spindle: Theories, and now experiments. BioEssays, 39(2), 


\section{REFERENCES}

1600212. Retrieved from http://www.ncbi.nlm.nih.gov/pubmed/28026040 doi: 10.1002/bies.201600212

Wu, W., Dong, M.-Q., Wu, X.-G., Sun, H.-Y., Tse, H.-F., Lau, C.-P., \& Li, G.-R. (2012, feb). Human ether-à-go-go gene potassium channels are regulated by EGFR tyrosine kinase. Biochim. Biophys. Acta, 1823(2), 282-9. Retrieved from https://linkinghub.elsevier.com/retrieve/pii/ S0167488911002990 doi: 10.1016/j.bbamcr.2011.10.010

Wu, X., Zhong, D., Gao, Q., Zhai, W., Ding, Z., \& Wu, J. (2013). MicroRNA-34a inhibits human osteosarcoma proliferation by downregulating ether à go-go 1 expression. Int. J. Med. Sci., 10(6), 676-682. Retrieved from http:// WwW.ncbi.nlm.nih.gov/pubmed/23569431 doi: 10.7150/ijms.5528

Xu, N., Luo, K. Q., \& Chang, D. C. (2003, jul). Ca2+ signal blockers can inhibit $\mathrm{M} / \mathrm{A}$ transition in mammalian cells by interfering with the spindle checkpoint. Biochem. Biophys. Res. Commun., 306(3), 737-45. Retrieved from http:// wWw.ncbi.nlm.nih.gov/pubmed/12810081

Yang, C., Hao, J., Kong, D., Cui, X., Zhang, W., Wang, H., .. Xu, B. (2014, sep). ATM-mediated Mad1 Serine 214 phosphorylation regulates Mad1 dimerization and the spindle assembly checkpoint. Carcinogenesis, 35(9), 20072013. Retrieved from http://www.ncbi.nlm.nih.gov/pubmed/24728176 doi: $10.1093 /$ carcin/bgu087

Yeh, C.-W., Yu, Z.-C., Chen, P.-H., Cheng, Y.-C., \& Shieh, S.-Y. (2014, may). Phosphorylation at Threonine 288 by Cell Cycle Checkpoint Kinase 2 (CHK2) Controls Human Monopolar Spindle 1 (Mps1) Kinetochore Localization. $J$. Biol. Chem., 289(22), 15319-15327. Retrieved from http://www.ncbi.nlm .nih.gov/pubmed/24764296 doi: 10.1074/jbc.M114.552273

Yellen, G. (2002, sep). The voltage-gated potassium channels and their relatives. Nature, 419(6902), 35-42. Retrieved from http://www.ncbi.nlm.nih.gov/ pubmed/12214225 doi: 10.1038/nature00978

Yoon, A.-r., Gao, R., Kaul, Z., Choi, I.-K., Ryu, J., Noble, J. R., ... Wadhwa, R. (2011, oct). MicroRNA-296 is enriched in cancer cells and downregulates p21WAF1 mRNA expression via interaction with its 3' untranslated region. Nucleic Acids Res., 39(18), 8078-8091. Retrieved from http://www.ncbi.nlm 
.nih.gov/pubmed/21724611 doi: 10.1093/nar/gkr492

Zachos, G., Black, E. J., Walker, M., Scott, M. T., Vagnarelli, P., Earnshaw, W. C., \& Gillespie, D. A. (2007, feb). Chk1 is required for spindle checkpoint function. Dev. Cell, 12(2), 247-260. Retrieved from http://www.ncbi.nlm.nih.gov/ pubmed/17276342 doi: 10.1016/j.devcel.2007.01.003

Zetterberg, A., \& Larsson, O. (1985, aug). Kinetic analysis of regulatory events in G1 leading to proliferation or quiescence of Swiss 3T3 cells. Proc. Natl. Acad. Sci. U. S. A., 82(16), 5365-9. Retrieved from http://www.ncbi.nlm.nih.gov/ pubmed/3860868

Zhang, Q., \& Yang, H. (2012). The Roles of VHL-Dependent Ubiquitination in Signaling and Cancer. Front. Oncol., 2, 35. Retrieved from http:// www.ncbi.nlm.nih.gov/pubmed/22649785 doi: 10.3389/fonc.2012.00035

Zhang, S., Chang, L., Alfieri, C., Zhang, Z., Yang, J., Maslen, S., ... Barford, D. (2016). Molecular mechanism of APC/C activation by mitotic phosphorylation. Nature, 533(7602), 260-264. Retrieved from http://www.ncbi.nlm.nih.gov/ pubmed/27120157 doi: 10.1038/nature17973

Zhang, S., Hemmerich, P., \& Grosse, F. (2007, may). Centrosomal localization of DNA damage checkpoint proteins. J. Cell. Biochem., 101(2), 451-465. Retrieved from http://www.ncbi.nlm.nih.gov/pubmed/17171639 doi: 10.1002/jcb.21195

Zhao, H., \& Piwnica-Worms, H. (2001, jul). ATR-Mediated Checkpoint Pathways Regulate Phosphorylation and Activation of Human Chk1. Mol. Cell. Biol., 21(13), 4129-4139. Retrieved from http://www.ncbi.nlm.nih.gov/pubmed/ 11390642 doi: 10.1128/MCB.21.13.4129-4139.2001

Zheng, N., Wang, Z., \& Wei, W. (2016, apr). Ubiquitination-mediated degradation of cell cycle-related proteins by F-box proteins. Int. J. Biochem. Cell Biol., 73, 99-110. Retrieved from http://www.ncbi.nlm.nih.gov/pubmed/26860958 doi: 10.1016/j.biocel.2016.02.005

Ziechner, U., Schönherr, R., Born, A.-K., Gavrilova-Ruch, O., Glaser, R. W., Malesevic, M., ... Heinemann, S. H. (2006, mar). Inhibition of human ether à go-go potassium channels by $\mathrm{Ca} 2+/$ calmodulin binding to the cytosolic N- and C-termini. FEBS J., 273(5), 1074-86. Retrieved from http://doi.wiley.com/10.1111/j.1742-4658.2006.05134.x doi: 
$10.1111 / \mathrm{j} .1742-4658.2006 .05134 . \mathrm{x}$

Zieve, G. W., Turnbull, D., Mullins, J. M., \& McIntosh, J. R. (1980, apr). Production of large numbers of mitotic mammalian cells by use of the reversible microtubule inhibitor nocodazole. Nocodazole accumulated mitotic cells. Exp. Cell Res., 126(2), 397-405. Retrieved from http://www.ncbi.nlm.nih.gov/pubmed/ 6153987

Zou, L., \& Elledge, S. J. (2003). Sensing DNA damage through ATRIP recognition of RPA-ssDNA complexes. Science (80-. )., 300 (5625), 1542-1548.

Zwetsloot, A. J., Tut, G., \& Straube, A. (2018, dec). Measuring microtubule dynamics. Essays Biochem., 62(6), 725-735. Retrieved from http://www.ncbi .nlm.nih.gov/pubmed/30287587 doi: 10.1042/EBC20180035 
ApPEndix

\section{Appendix}


Appendix 


\section{$R$ code for calcium data processing}

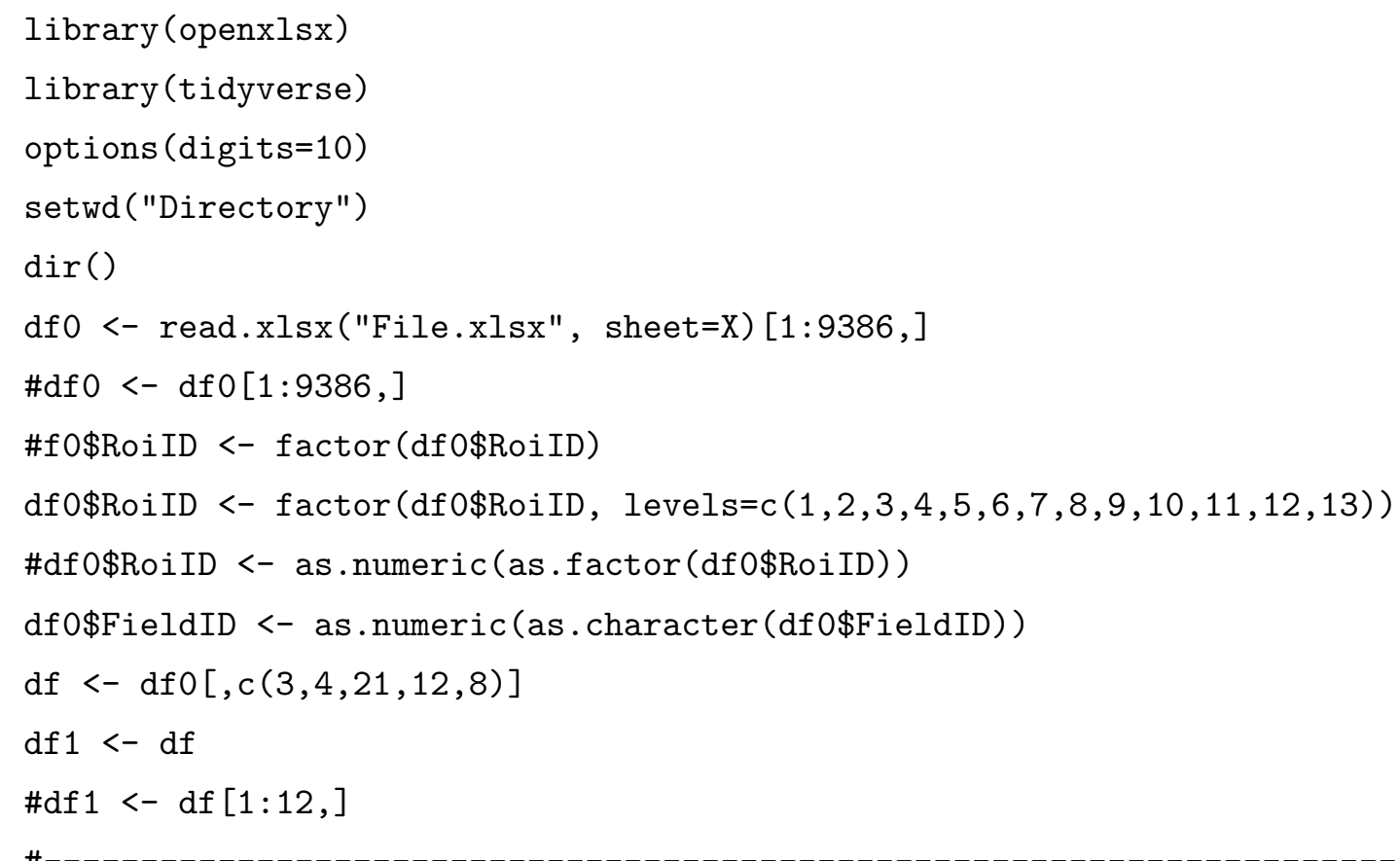

library(reshape2) data_sum <- dcast(df1, FieldID + AcqTime RoiID, value.var="SumIntensity", fun.aggregate $=$ mean)

data_area <- dcast(df1, FieldID + AcqTime RoiID, value.var="ROIArea", fun. aggregate $=$ mean)

colnames(data_sum) <- c("Time ID", "Time, sec", "Sum Intensity 1", "Sum Intensity 2", "Sum Intensity 3", "Sum Intensity 4", "Sum Intensity 5", "Sum Intensity 6", "Sum Intensity 7", "Sum Intensity 8", "Sum Intensity 9", "Sum Intensity 10", "Background Mean Intensity 11 ", "Background Mean Intensity 12", "Background Mean Intensity 13") colnames (data_area) <- c("Time ID", "Time, sec", "ROI Area 1", "ROI Area 2", "ROI Area 3", "ROI Area 4", "ROI Area 5", "ROI Area 6", "ROI Area 7", "ROI Area 8", "ROI Area 9", "ROI Area 10", "Background Mean Intensity 11", "Background Mean Intensity 12", "Background Mean Intensity 13") df <cbind (data_sum, data_area $[,-c(1,2,13,14,15)]$ ) df <- $d f[, c(1,2,3,16,4,17,5,18,6,19,7,20,8,21,9,22,10$, $23,11,24,12,25)]$

\#\#\# Sum of Sum Intensity \& Sum of ROI Area:

df $1<-d f$

df1\$"Sum of Sum Intensity" <- rowSums (df $[, c(3,5,7,9,11,13,15,17$, $19,21)]$ )

df1\$"Sum of ROI Area" <- rowSums (df $[, c(4,6,8,10,12,14,16,18,20$, 22)])

\#\#\# Mean Intensities for Background ROIs: 


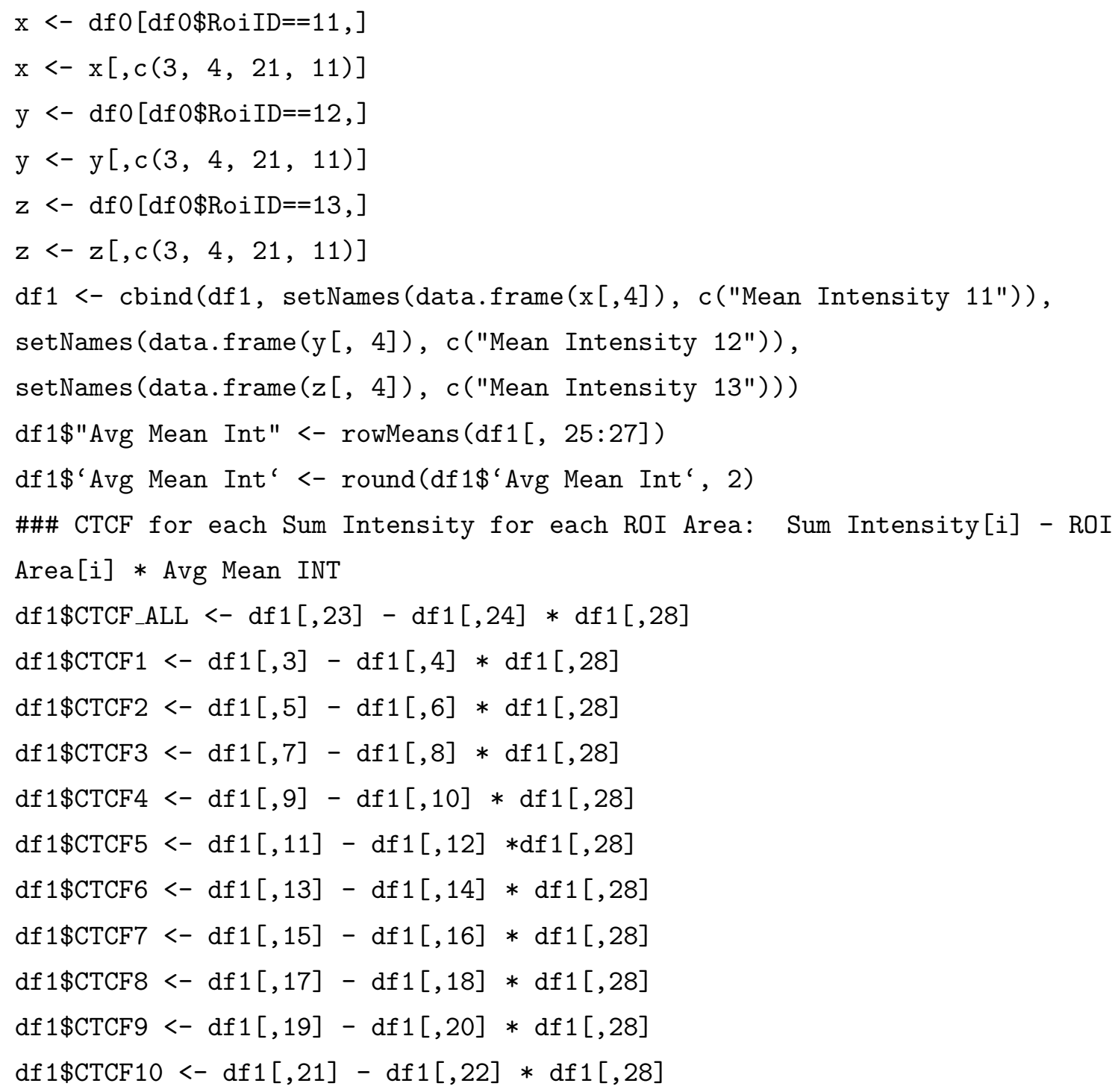




\section{MATLAB code for calcium oscillations analysis}

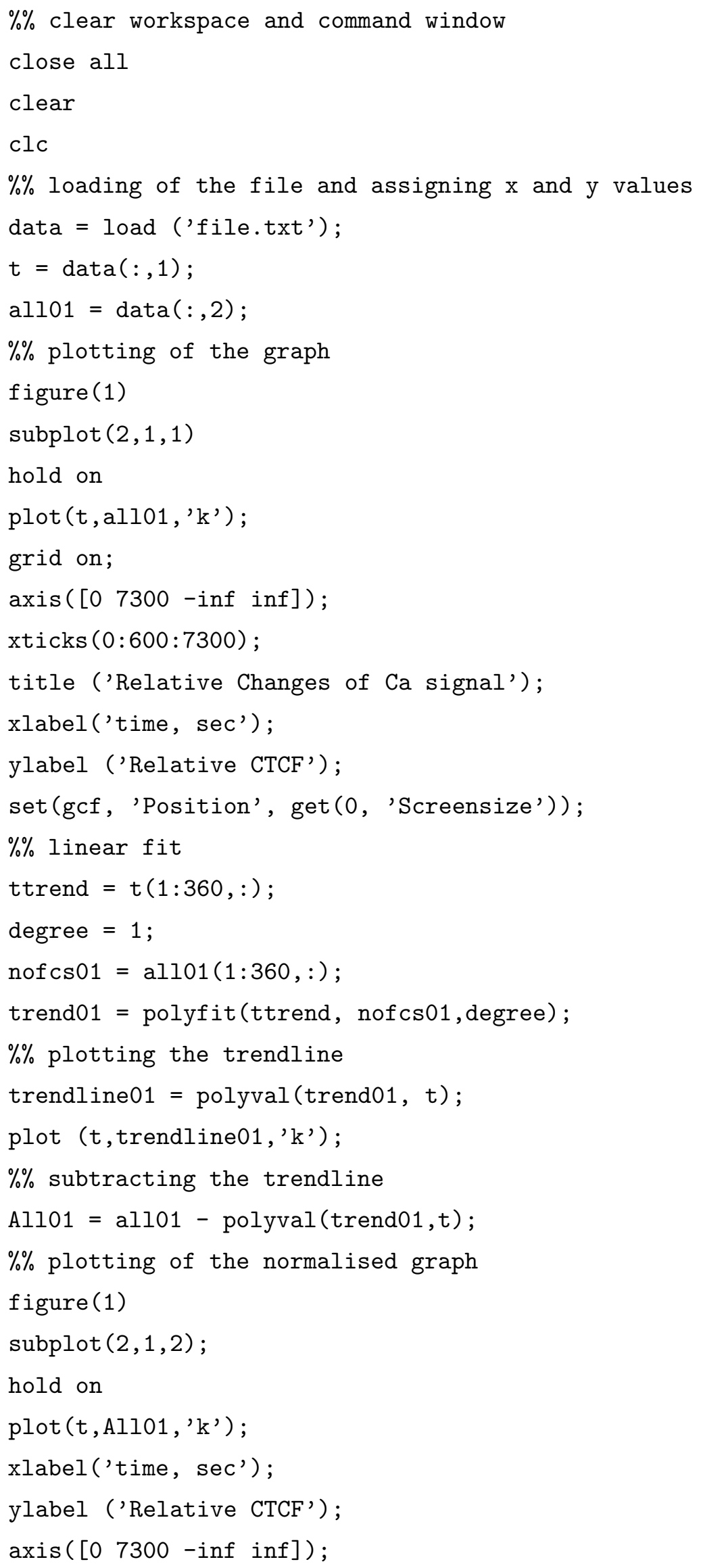




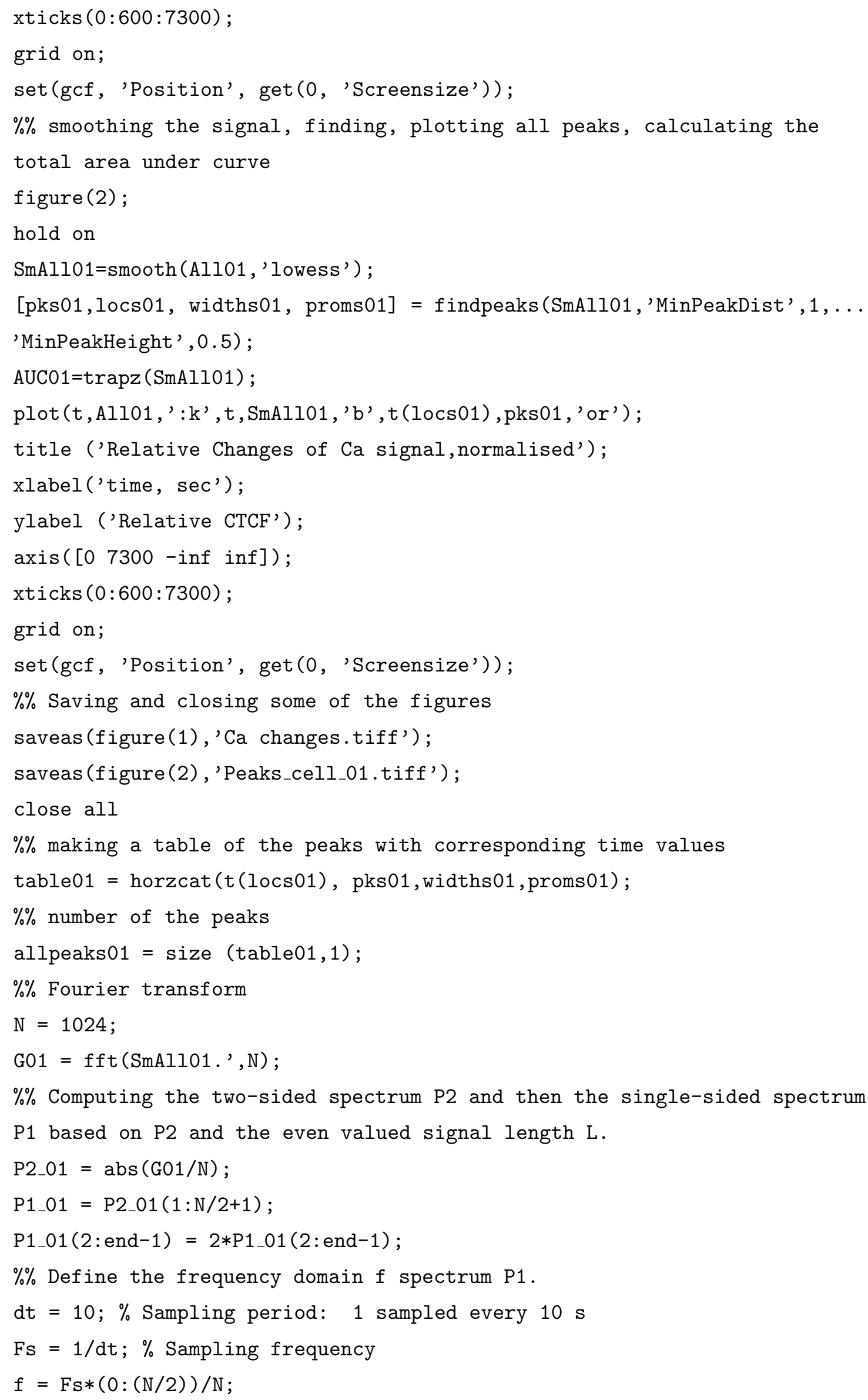


single-sided amplitude, then subplotting the histogram and calculating the counts and centers

figure (3);

hold on

AUCP1_01=trapz (P1_01); \%\#ok<*NASGU>

[pksP1_01, locsP1_01, widthsP1_01, promsP1_01] =

findpeaks (P1_01, 'MinPeakDist' ', 1, . .

'MinPeakHeight', 0.5);

subplot $(2,1,1)$

plot (f,P1_01, 'k', f (1ocsP1_01), pksP1_01, 'or') ;

title ('Single-Sided Amplitude Spectrum of relative changes of intracellular Calcium(t)');

xlabel ('Frequency, $\mathrm{mHz}$ ');

ylabel('|P1(f)|')

grid on;

set(gcf, 'Position', get(0, 'Screensize'));

figure (3)

hold on

[countsP1_01, centersP1_01] = hist $($ pksP1_01); \%\#ok<*ASGLU>

subplot $(2,1,2)$

nbins $=20$;

hist (pksP1_01, nbins);

$\mathrm{h}=$ findobj(gca, 'Type', 'patch');

h. FaceColor $=\left[\begin{array}{lll}0 & 0.5 & 0.5\end{array}\right]$;

h. EdgeColor = 'w';

grid on

title('Frequency of Single-Sided Amplitudes')

xlabel ('bins')

ylabel ('counts')

set(gcf, 'Position', get(0, 'Screensize'));

$\%$ saving figure

saveas (figure (3), 'Frequency_01.tiff');

close all

$\%$ making a table of the peaks with corresponding time values

tableP1_01 = horzcat $(f($ locsP1_01).',

pksP1_01. ', widthsP1_01.' ,promsP1_01.');

$\%$ making a table of the counts and centers

centersandbinsP1_01=horzcat (countsP1_01.' , centersP1_01.');

$\% \%$ number of the peaks

allpeaksP1_01 = size $($ tableP1_01,1); 
MATLAB CODE FOR CALCIUM OSCILLATIONS ANALYSIS

$\%$ write all the tables

$\%$ clearing the workspace

close all

clear

$\mathrm{clc}$ 